$40 \cos 2=0$

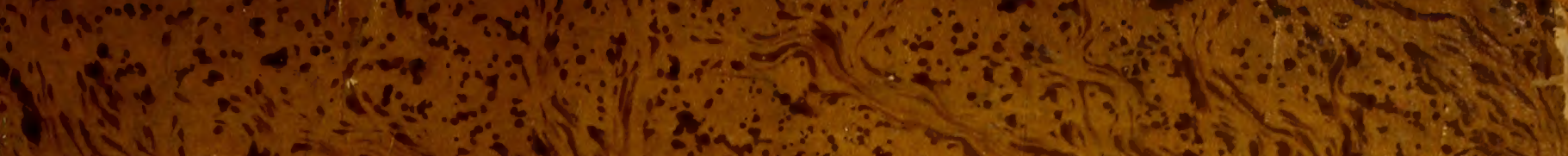

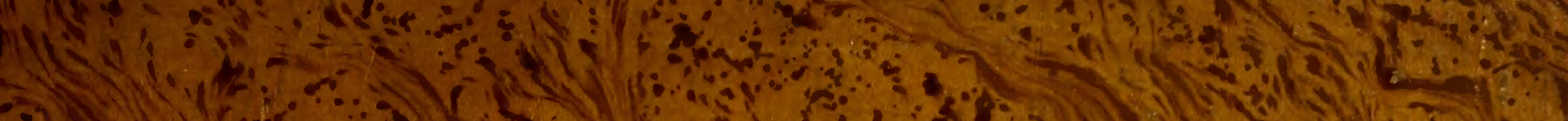

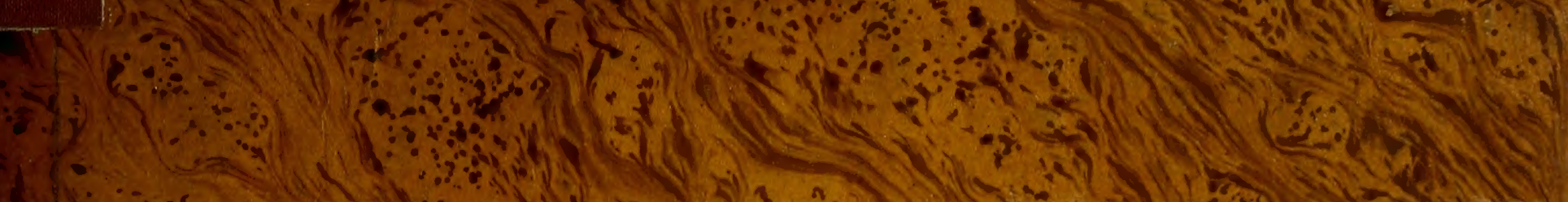

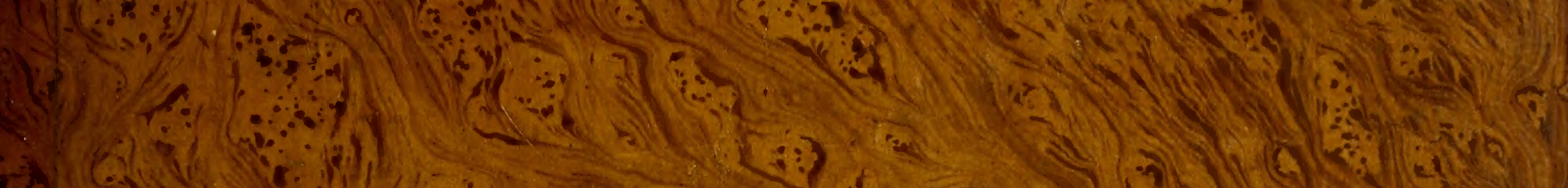

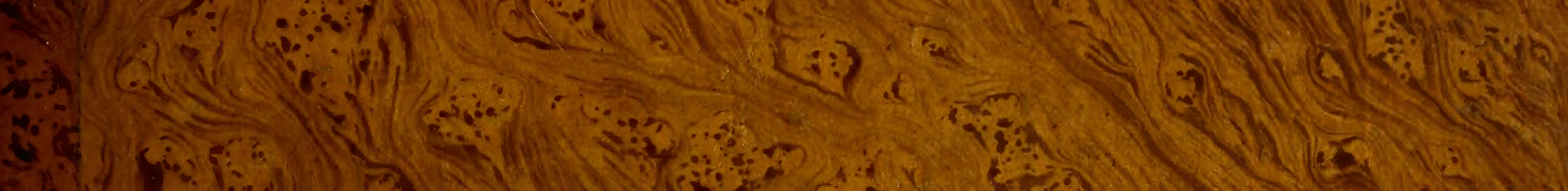

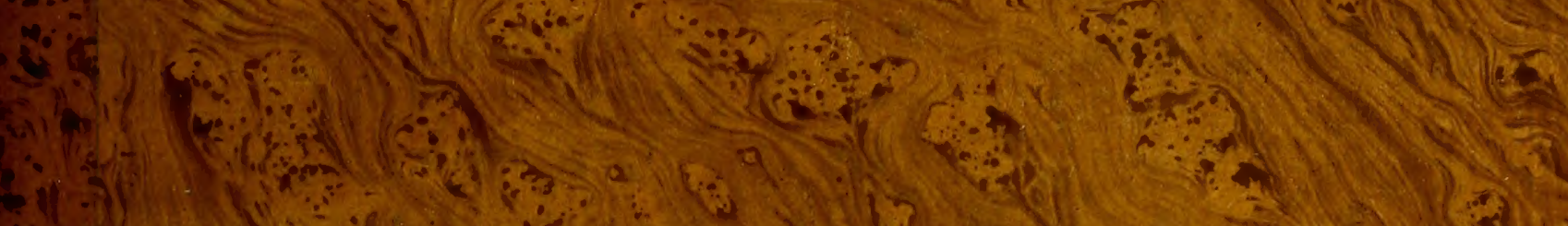

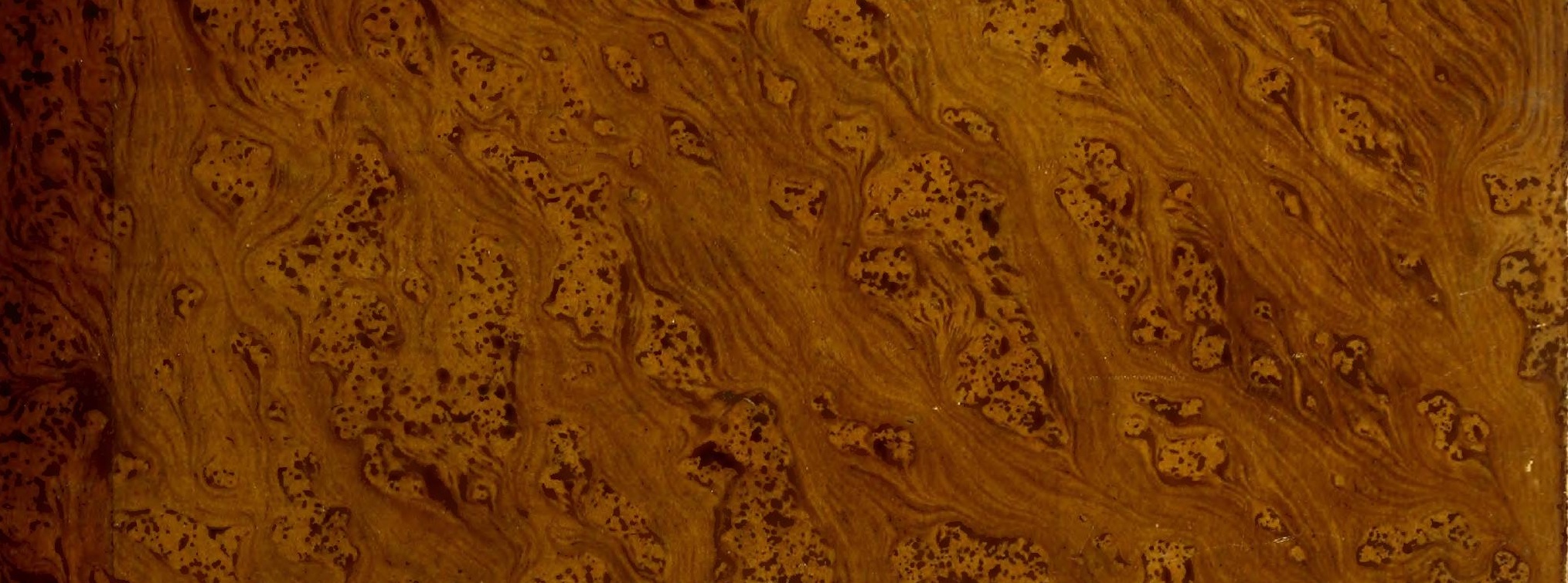

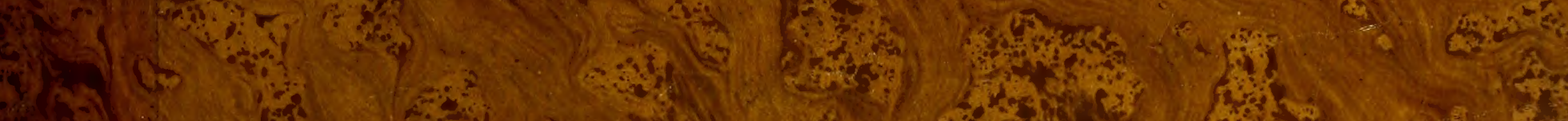

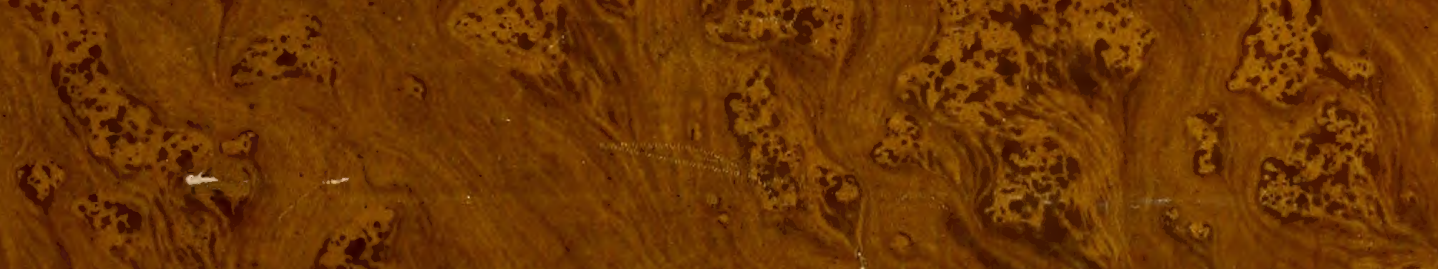

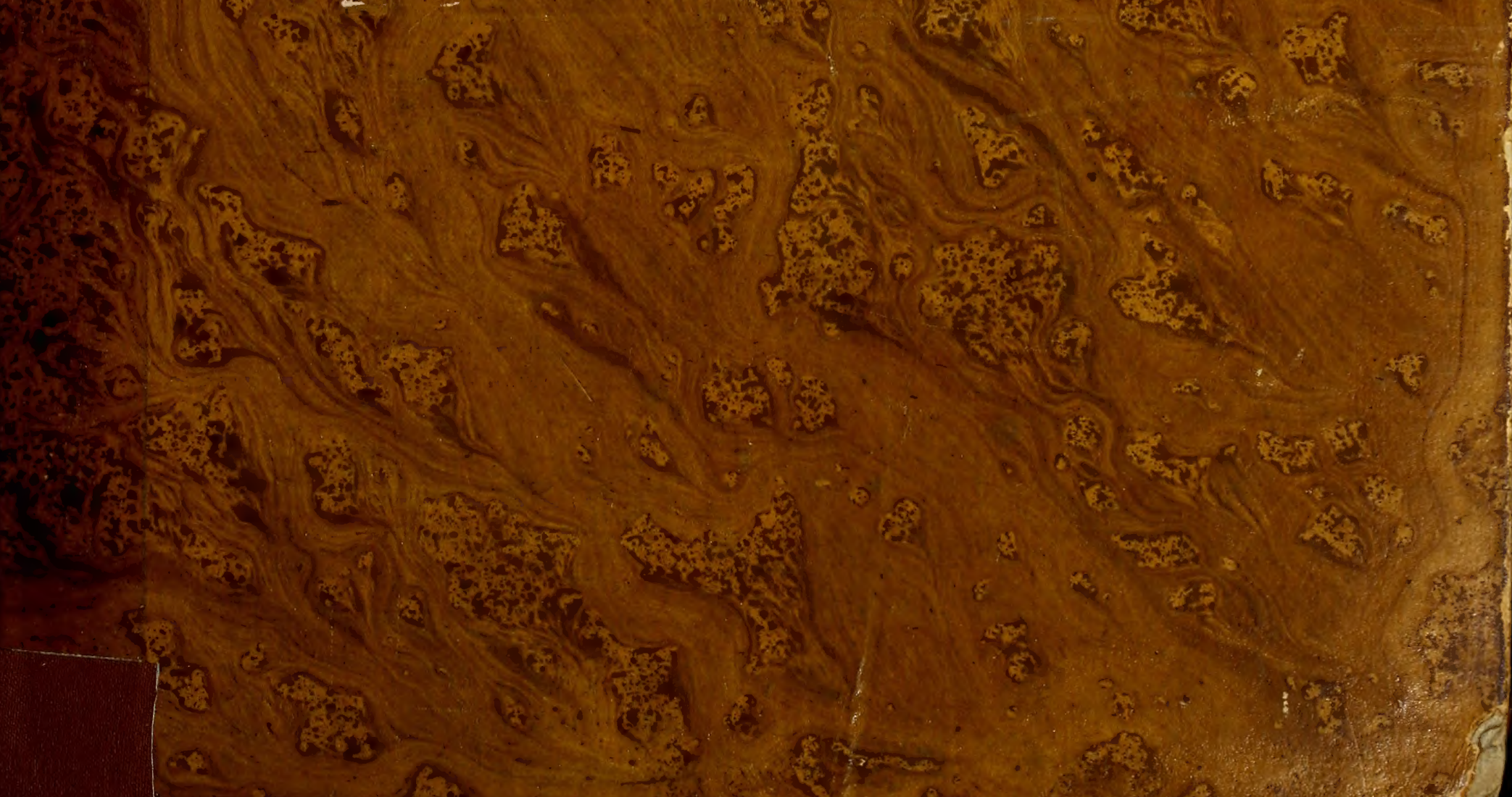




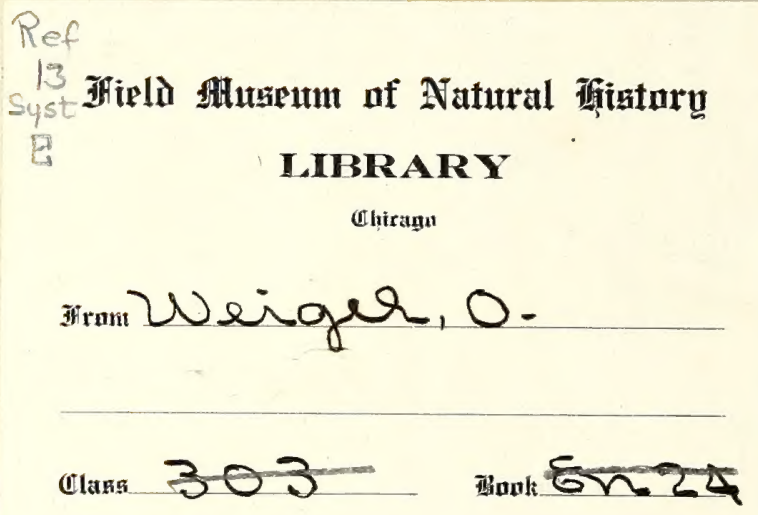





\section{ICONOGRAPHIA}

\section{GENERUM PLANTARUM}

E D I D II T

\section{STEPHA NUS ENDLICHETR}

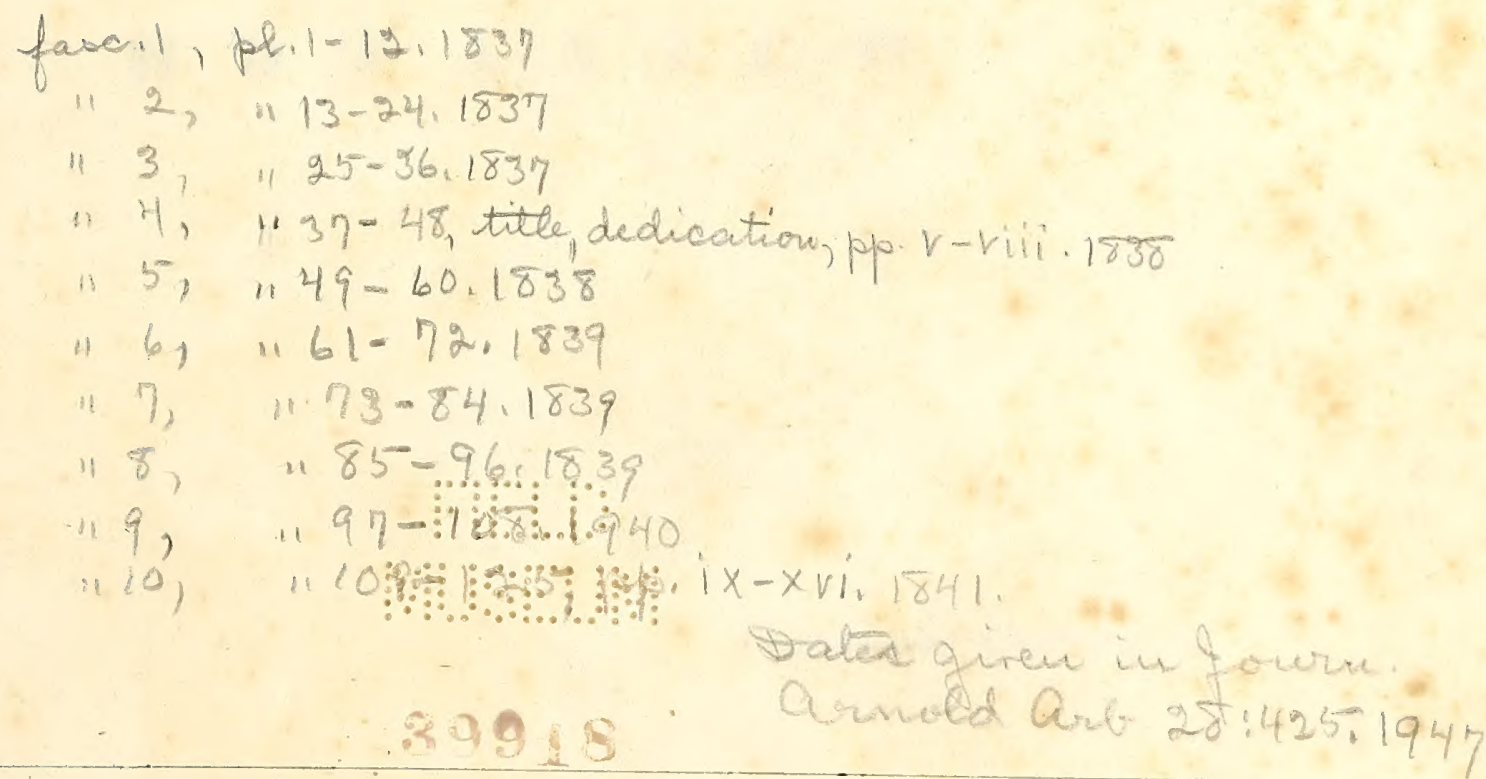

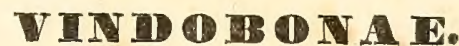

A PUD FR. BECI, UNIVERSITATIS BIBLIOPOLAM.

11용. 



\title{
ALLANO CUNNINGHAM
}

\section{SERENISSIMAE BRITANNIARUM REGINAE}

\author{
- IN COLONIIS AUSTRALIBUS
}

I I T N I C I

D I C A T 


\section{Digitized by the Internet Archive in 2013}




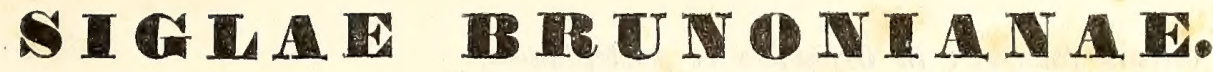

a. Flos ante expansionem.

a 1. Flos expansus.

b. Operculum floris : e calyce et corolla confluentibus saepius formatum.

c. Perigonium: integumentum florale monocotyledonum, et utplurimum simplex dicotyledonum (corolla Linn. calyx Juss.).

c 1. Perigonii foliola v. laciniae seriei exterioris; saepe calycinae indolis (calyx Linn.).

c 2. Perigonii foliola v. laciniae seriei interioris (exceptis $c$ 3. et 4.); saepe texturae petaloideae (corolla Linn.).

c 3. Labellum ejusve appendices. In Orchideis.

c 4. Squamulae hypogynae Graminum (nectarium Linn.).

c 5. Appendices perigonii.

d. Calyx.

e. Corolla monopetala.

e 1. Petala.

e 2. Appendices corollae (nectarium Linn. parapetala Ehrhart).

f. Discus hypogynus v. epigynus.

$f$ 1. Glandulae v. squamulae hypogynae v. epigynae. 
g. Columna genitalium: Orchidearum, stylidearum.

g 1. Genitalia distincta: integumentis remotis.

h. Stamina.

$h$ 1. Anthera.

h 2. Pollen.

$h$ 3. Pollinis massae: in Orchideis et Asclepiadeis.

h 4. Stamina sterilia.

$h$ 5. Corona tubi staminei : in Asclepiadeis (nectarium Linn.).

i. Pistillum.

$i$ 1. Ovarium.

$i$ a. Stylus.

$i$ 2. Stigma.

i 3. Stigmatis indusium: in Goodenoviis, Brunonia.

i 4. Ovulum.

i $\beta$. Chalaza.

i $\gamma$. Micropyle.

i $\delta$. Rhaphe.

l. Fructus compositus: floribus pluribus communis.

$l$ 1. Pericarpia plura distincta: floris unici.

m. Induviae. Reliquiae foliis fructum $v$, augentes v. coronantes v, eidem adnatae.

$m$ 1. Pappus.

$m$ 2. Calyptra Muscorum.

n. Pericarpium. Complectens ejusdem species omnes a simplicissima caryepside Graminum.

$n$ 1. Pericarpium apertum.

$n$ 2. Dissepimentum.

$n$ 3. Valvulae. 
n 4. Operculum.

n 5. Peristomium Muscorum.

n 6. Placenta (receptaculum seminum Grert.).

$n$ 7. Funiculus umbilicalis.

$n$ 8. Strophiola v. caruncula umbilicalis.

n 9. Arillus.

o. Semen.

o 1. Ala seminis.

o 2. Coma seminis: in Asclepiadeis, Epilobio.

o 3. Integumenta seminis.

- 4. Albumen (perispermum Juss. endospermum Rich.).

o 5. Vitellus : in Scitamineis, Nymphaea.

p. Embryo.

$p$ 1. Cotyledon.

p 2. Plumula.

$p$ 3. Radicula.

g. Folium.

g 1. Petiolus.

g 2. Stipula.

r. Caulis v. scapi portio.

s. Inflorescentia: complectens species omnes; exceptis duabus sequentibus ( 1 1. et $s$ 2.).

$s$ 1. Flos compositus.

$s$ 2. Locusta Graminis (v, uniflora ${ }_{i}$ v. multiflora).

t. Involucrum umbellae, capituli.

t 1. Involucrum floris compositi (calyx communis Linn.).

$t$ 2. Gluma Graminum (calyx Linn.). 
t 3. Calyx exterior: Malvacearum, Dipsaceamum, Brunoniae.

4. Involucrum (sori v. capsulae unicae) Filicum (indusium Swartz).

$t$ 5. Bracteae.

औ6. Squamae amenti.

$t$ \%. Paleae.

8. Paraphyses Muscorum.

t 9. Calyptra: dum e bracteis connatis formata.

$u$. Receptaculum floris unici.

$\boldsymbol{u}$ 1. Receptaculum commune v. floris compositi v. amenti v. capituti.

$x$. Diagramma floris.

Signa sequentia literis subposita sic intelligenda.

* Partem vi expansam v. apertam esse denotat.

+.... sectam verticaliter.

$\because \ldots . .$. horizontaliter.

-... magnitudine diminutam.

Dum partes magnitudine auctae sunt, literae majusculae pro minoribus substituuntur. 


\section{Comspectus distogitiomis tabularumb.}

\section{Gasteronanceres.}

357. A scröe pentactina Endl, Atakt, t. 50, - Habitat in Nova Hollandia. (Ferd, Bauer.) I. 1.

\section{Hyeopodiaceae.}

695. Psilotum (Tmesipteris) Forsteri Lindl. Flor. Norf 6. Tmesipteris tannensis Bernhardi in Schrader Jour. 1801 I. 131. non Labill. - Habitat in insula Nor folk. (Forster Ferd. Bauer.) VIII. 85.

\section{CHannimeae.}

882. Coelachne pulchella R. Brown Prodr, 18\%, - Habitat in Nova Hollandia tropica. (Ferd. Bauer.) I. 2.

\section{Cyperaceac.}

963. Diplacrum caricinum R. Brown Prodr. 240. Habitat in Nova Hollandia tropica. (Ferd. Bauer.) IIl. 25 .

\section{Centrolepideae.}

1006. Centrolepis fascicularis Labillard. Nov. Holl. 1.7. t. 1. Desvauxia Billardierii R. Brown Prodr. 252.Habitat in Nova Hollandia orientali et in insula Diemen. (Labillard, R. Brown, Ferd. Bauer, Sieber, Hügel.) V. 49.

\section{Xyrideac.}

1025. Xyris (Pomatoxyris) operculata Labill. Nov. Holl. I.] 14. t 10. R. Brown Prodr. 257. - Habitat in Nova Hollandia orientali et in insula Diemen. (Labillard, R. Brown, Ferd. Bauer.) II. 13.

\section{Evitomaceac.}

1045. Hydrocleis Commersonii Richard in Mem. Mus. I. 368. - Habitat in America tropica. IV. 37.

1046. Limnocharis Plumierii Richard in Mem. Mus, I. 370. - Habitat in America tropica. 1II. 26.

\section{Calectasieae.}

1059. Calectasia cyanea R. Brown Prodr. 263. - Habitat in Nova Hollandia austro-occidentali. (R Brown, Ferd. Bauer, Hügel.) IV. 38.

\section{Melamtlnaceae.}

1072. Anguillaria dioica $R$. Brown Prodr. 273. - Habitat in Nova Hollandia orientali et insula Diemen. (R. Brown, Ferd. Bauer, Hügel.) I. 3.

\section{耳ํํำaceมอ.}

1104. Blandfordia nobilis Smith Exot. Bot. I. 5, t. 4. R. Brown Prodr. 296. - Habitat in Nova Hollandia orientali, (R. Brown, Ferd. Bauer, Sieber, A. Cun ningham, Hügel.) 1II. 27.

1143. Tricoryne elatior R. Brown Prodr. 278. - Habitat in Nova Hollandia orientali. ( $R$. Brown, Ferd Bauer, A. Cunningham.) VI. 61.

1162. Eustrephus latifolius R. Brown Prodr. 281. Habitat in Nova Hollandia orientali et tropica. $(\bar{H}$ Brown, Ferd. Bauer.) I. 4.

1164. Arthropodium paniculatum $\boldsymbol{R}$. Brown Prodr, 276 . Anthericum paniculatum Andrews Bot. Reposit. t. 395. Anthericum milleflorum Redoute Liliac. 58. Habitat in Nova Hollandia orientali. (Labill., $\boldsymbol{R}$. Brown, Ferd.Bauer, Sieber, A. Cunningham, Hügel.) III. 28 ,

1169. Laxmannia gracilis R. Brown Prodr. 286. - Ha bitat in Nova Hollandia orientali. (R. Brown, Ferd.Bauer, Sieber, A. Cunningham, Hügel.) IX.97.

\section{Sminillaceac.}

1184. Smilax glycyphylla Smith in Whités Voy, 230 . R Brown Prodr. 293. - Habitat in Nova Hollandia orientali. (White, R. Brown, Ferd. Bauer, Hügel.) IV: 39 .

\section{Trideac.}

1234. Patersonia glauca R. Brown Prodr. 403. Genosiris fragilis Labillard. Nov. Holl. 304. - Habitat in Nova Hollandia orientali et in insula Diemen. (Labillard., R. Brown, Ferd. Bauer, Sieber, A. Cunningham, Hügel.) V. 50.

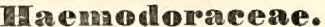

1253. Haemodorum spicatum R. Brown Prodr. 300. Habitat in Nova Hollandia australi. (R. Brown Ferd, Bauer.) IX. 98.

1259. Tribonanthes australis Endl. Nov. Stirp. Mus Vind. Dec. n, 34. - Habitat in Novae Hollandiae au stro-occidentalis colonia King. Georges - Sound. (Hügel.) X. 109. 


\section{Drelhideae.}

1369. Dendrobium teretifolium R. Brown Prodr. 333. - Habitat in Nova Hollandia orientali. (R.Brown, Ferd. Bauer, Hügel.) IX. 99.

1534. Bartholina pectinata R. Brown in Aiton Hort. kew. ed. 2. V. 194. Lindley Orchid. 333. Orchis pectinata Willd. Spec. IV. 11. - Habitat in Capite bonae spei (Ferd. Bauer.) IV. 49.

1544. Gastrodia sesamoides $R$. Brown Prodr. 330. Habitat in Nova Hollandia orientali. (R. Brown, Ferd. Bauer.) I. 5.

1579. Cryptostylis longifolia $R$. Brown Prodr. 317. Malaxis subulata Labillard. Nov. Holl.11. 62. t. 212. Habitat in Nova Hollandia et in insula Diemen. (Labillard, R. Brown, Ferd. Bauer, Hügel.) II. 14.

1580. Calochilus paludosus R. Brown Prodr. 320. - Habitat in Nova Hollandia orientali. (R. Brown, Ferd. Bauer.) II. 17.

1588. Microtis parviflora R. Brown Prodr. 321.- Habitat in Nova Hollandia orientali, australi et tropica. (R. Brown, Ferd.Bauer, Sieber, A. Cunningham, Hügel.) II. 15.

1589. Acianthus fornicatus R. Brown Prodr. 321. - Habitat in Nova Hollandia orientali. (R. Brown, Ferd. Bauer, Sieber, A. Cunningham, Hügel.) II. 16.

1592. Eriochilus autumnalis R. Brown Prodr. 323. Epipactis cucullata Labillard. Nov. Holl. II. 61, t. 211. f. 2. - Habitat in Nova Hollandia orientali, australi et tropica. (R. Brown, Ferd. Bauer, A. Cunningham, Hügel.) I. 6.

1593. Caladena carnea $R$. Brown Prodr. 324. - Habitat in Nova Hollandia orientali et tropica, R.Brown, Ferd. Bauier, A. Cunningham.) V. 51.

1594. Glossodia major R. Brown Prodr. 326. - Habitat in Nova Hollandia orientali. (R.Brown, Ferd.Bauer.) VI. 41.

1596. Lyperanthus nigricans $\boldsymbol{R}$. Brown Prodr. 325. Habitat in Nova Hollandia orientali et australi. ( $R$. Brown, Ferd. Bauer, Hügel.) 1. 7.

1597. Corysanthes (Steleocorys) unguiculata $R$. Brown Prodr. 328. - Habitat in Nova Hollandia orientali. (Ferd. Bauer.) II. 13.

1598. Caleya major R. Brown. Caleana major R. Brown Prodr. 329. - Habitat in Nova Hollandia orientali. (R. Brown, Ferd. Bauer.) I. 8.

1616. Thelychiton argyropus Endlicher Prodr. Flor. Norf. n. 72. - Habitat in insula Norfolk. (Ferd. Bauer.) II. 29.

\section{Minsaceae.}

1650. Ravenala madagascariensis Sonner. Voy. II. 223. t. 124. Jacq. Hort. Schönbr. I. 47. t. 93. Urania Ravenala Richard Musac, 13, $t, 5-6$, - Habitat in Madagascar. IV. 42.

\section{Najadeae.}

1664/1. Cycnogeton Hügelii Endlicher in Annal. Wiener Mus. II. 210. Nov. Stirp. Mus. Vindob. Dec. n.189. Habitat in Novae Hollandiae colonia Swan - River. (Hügel.) VII. 73.

\section{Aroideae.}

1707. Gymnostachys anceps R. Brown Prodr. 337. Habitat in Nova Hollandia orientali. (R. Brown, Ferd. Bauer, Sieber, A. Cunningham, Hügel.) I. 9.

\section{Urticaceae.}

1884. Böhmeria australis Endlicher Flor. Norf. n. 82. - Habitat in insula Norfolk. (Ferd. Bauer, A. Cunningham.) VIII. 86.

\section{Putronjiveae.}

1894. Putranjiva Roxburghii Wallich Tent. Flor. nep. 16. Royle Himalay. 347. t. 100. Nageja Putranjiva Roxburgh Flor. Ind. or. III. 766. - Habitat in montibus Indiae. (Wallich.)

\section{Chemopodeae.}

1920/1. Didymanthus Roëi Endlicher Nov. Stirp. Decad. n. 10. - Habitat in Novae Hollandiae austro - occidentalis interioribus (Roë.) XI. 100.

\section{Amaramtaceae.}

1967. Centrostachys aquatica Wallich in Roxburgh Flor. Ind. II. 497. Achyrantes aquatica Roxburgh Flor. Ind. 1. 673. - Habitat in India orientali. (Wallich.) II. 20.

1978. Deeringia celosioides $R$. Brown Prodr. 413. Celosia baccata Retz Observ. 5. 23. - Habitat in Asia tropica. - In Nova Hollandia tropica et orientali. (R. Brown, Ferd. Bauer.) VI. 62.

\section{Polygoneae.}

1986. Polygonum australe A. Richard Flor. Nov. Zeel. 178. Coccolaba australis Forst. Prodr. n. 179. Crescit in Nova Zeelandia (Forst.) et in insula Norfolk. (Ferd. Bauer.) VII. 87.

\section{Nyetagineae.}

2012. Pisonia grandis R. Brown Prodr. 422. - Habitat in Nova Hollandia tropica. (R. Brown, Ferd. Bauer.) III. 30 .

\section{Tlomimiaceae.}

2019. Ruizia fragrans Pav. Syst. $\mathrm{Fl}$. peruv, 266. Peumus fragrans Persoon Encheir. II. 624. - Habitat in Chili. (Pöppig, Bridges.) II. 21.

2022. Doryphora Sassafras Endl. Atherosperma Sassafras A.Cunningham mse. - Habitat in Nova Hollandia orientali. (A. Cunningham.)

\section{Taurineae.}

2066. Litsaea Baueri Endl. msc. - Habitat in Nova Hollandia orientali. (Ferd. Bauer.) IV. 44 


\section{Gyroenrpeac.}

2068. Gyrocarpus sphenopterus $\boldsymbol{R}$. Brown Prodr. 261. - Habitat in Nova Hollandia tropica. (Ferd.Bauer.) IV. 43.

\section{Samtalaceae.}

2074. Choretrum glomeratum R. Brown Prodr. 354. - Habitat in Nova Hollandia australi. (R. Brown, Ferd. Bauer.) IV. 45.

2075. Leptomeria (Oxymeria) acida R. Brown Prodr. 253. Habitat in Nova Hollandia orientali. (R. Brown, Ferd. Bauer, Sieber, Hügel.) VII. 74.

\section{Da plnnoideae.}

2098. Pimelea (Thecanthes) punicea R. Brown Prodr. 359. - Habitat in Nova Hollandia tropica. $(R$. Brown, Ferd. Bauer.) I. 11.

2105. Wikströmia australis Endl. Prodr. Flor. Norf. n. 92 - Habitat in insula Norfolk. (Ferd. Bauer.) II. 22

\section{Protenceae.}

2130. Adenanthos terminalis $\boldsymbol{R}$. Brown Prodr. 367. Habitat in Nova Hollandia australi. (Labillard, $R$. Brown, Ferd. Bauer, Hügel.) X. 110.

2131. Synaphaea dilatata R. Brown Prodr. 370. Conospermum reticulatum Smith in Rees. Cyclop. - Habitat in Nova Hollandia australi. (Ferd. Bauer, R. Brown.) III. 31.

2132. Conospermum (Euconospermum) ericifolium $S$ mith in Rees Cyclop. R. Brown Prodr. 368. - Habitat in Nova Hollandia orientali. (R. Brown, Ferd. Bauer.) III. 31 .

- Conospermum (Chilurus) teretifolium R. Brown Prodr. 369. - Habitat in Nova Hollandia australi. (R. Brown, Ferd. Bauer, Hügel.) IV. 46.

2133. Stirlingia anethifolia Endl. msc. Simsia anethifolia R. Brown Prodr. 368. - Habitat in Nova Hollandia australi. (Ferd. Bauer, Hügel.) II. 23.

2134. Franklandia fucifolia $\boldsymbol{R}$. Brown Prodr. 266. Habitat in Nova Hollandia australi. (R. Brown, Ferd. Bauer, Hügel.) V. 52.

2135. Symphyonema paludosum R. Brown Prodr. 371. - S. montanum sphalm. in tab. - Habitat in Nova Hollandia orientali. (R. Brown, Ferd. Bauer, Sieber, Hügel.) 1. 12.

2143. Grevillea (Lissostylis) riparia R. Brown Prodr. 377. - Habitat in Nova Hollandia orientali, (R. Brown, (Ferd. Bauer.) III. 33.

2144. Hackea acicularis Knight et Salisb. Prot. 107. $\boldsymbol{R}$ Brown Prodr. 383. Conchium aciculare Vent. Mal mais. t. 111. - Habitat in Nova Hollandia orientali. (R. Brown, Ferd. Bauer.) II. 24.

2146. Xylomelum pyriforme Smith in Linn. Transact. IV. 214. R. Brown Prodr. 387. Banksia pyriformis Gärtner 1. 220. t. 47. f. 1. Hakea pyriformis $\mathrm{Ca}$ vanill. Ic. VI. 25. t. 538. - Habitat in Nova Hol- landia orientali. (R. Brown, Ferd. Bauer, Sieber, Hügel.) VI. 47, 48.

\section{Compositae.}

2213. Hoplophyllum spinosum DC.Prodr. V. 74. Ptero nia spinosa Linn. f. Supplem. 357. - Habitat in Capite bonae spei. (Thunb. Drége, Eckl. et Zeyh.) III. 34.

2374. Fresenia leptophylla DC. Prodr. V. 328. - Ha bitat in Capite bonae spei. (Drége.) III. 35.

2502. Wollastonia Forsteriana DC. Prodr. V. 548. We delia Forsteriana Endl, Prodr. Flor. Norf. 51. Buphthalmum uniflorum Forst. Prodr. n. 541. - Habitat in insula Norfolk. (Forst., Ferd.Bauer, A. Cunningham.) VIII. 88.

2629. Madaria corymbosa DC. Prodr. v. 692. - Habitat in America boreali - occidentali. (Douglas.) III. 36.

\section{Dobeliaceae.}

3062. Heterotoma lobelioides Zuccarini in Flora 1832. Beibl. p. 100. DC. Prodr. VII. 350. Myopsia mexicana Presl Lobeliac. 8. - Habitat in frigidissimis ad la Cumbre de San Antonio Mexicanorum, 8000 ped. supra mare. (Karwinsky.) V. 53.

\section{Trubiaceac.}

3105. Coprosma Baueri Endl. msc. Coprosma lucida Endl. Flor. Norf. n. 117. non Forst. - Habitat in insulae sinu Ausonis. (Ferd. Bauer, Hügel.)

3240. Hedyotis (Anotis) gentianoides Endl. msc. Honstonia caerulea Auct. - Habitat in America boreali. VIII. 89.

3275. Cosmibuena ochracea Endl. msc. Buena hexandra Pohl Plant. Brasil.I. 10.t. 8. - Habitat in Brasiliae provinciis Rio Janeiro et Minas-Geraes. (Schott, Pohl.) VIII. 90.

\section{Dlpaceae.}

3349. Olea (Gymnelaea) apetala Vahl Symb. III. 3. Endl. Prodr. Flor. Norf. 112. - Habitat in Nova Zeelandia (Forst.) et in insula Norfolk. (Ferd. Bauer, $A$. Cunningham.) V. 54.

3350. Notelaea ovata $R$. Brown Prodr. 380. - Habitat in Nova Hollandia orientali. (R. Brown, Ferd.Bauer, Sieber, Hügel.) V. 55.

\section{Loganiaceae.}

3362. Antonia pilosa Hooker Ic. $t$. 64. - Habitat ad fluvium Essequebo Guianae. (Schomburg.) V. 56.

3363. Coelostylis loganioides Torrey et $A$. Gray msc Endl. Nov. Stirp. Mus. Vindob. Dec, n. 41, - Habi tat in Florida occidentali prope Tampa Bay. ( $D r$. Burrows et M. Alden in Herb. Torrey.) XI. 101.

3364. a. Logania (Euosma) floribunda $R$. Brown Prodr. 456. Euosma albiflora Andrews Bot. Reposit. 520. Habitat in Nova Hollandia orientali. (R. Brown, Ferd. Bauer, Sieber, A. Cunningham, Hügel.) V.57. 
3364. b. Logania (Stomandra) pusilla R. Brown Prodr. 456. - Habitat in Nova Hollandia orientali. (R.) Brown, Ferd.Bauer.) V. 58.

\section{A TDCy}

3378. Melodinus 'Baueri Endl, Prodr, Flor. Norf. n. 113. - Habitat in insula Norfolk. (Ferd. Bauer, A Cunningham.) V. 59.

3426. Balfouria saligna $R$. Brown Prodr. 467. - Habitat in Nova Hollandia tropica. (R. Brown, Ferd. Bauer.) VII. 75 .

\section{Acletiateae.}

3444. Hybanthera biglandulosa Endl. Prodr. Flor. Norf. n. 115. - Habitat in insula Norfolk. (Ferd.Bauer.)

3456. Sarcostemma australe R. Brown Prodr. 463. Habitat in Nova Hollandia tropica et australi. $(R$ Brown, Ferd. Bauer.)

350t. Fockea capensis Endlicher Nov. Stirp. Mus. Vin. dob. Dec. n. 21. Cynanchum crispum Jacq. Fragm. bot. t. 36. f. 5. - Habitat in Capite bonae spei. (Hort. Schönbr.) VIII. 91.

3516. Microstemma tuberosum R. Brown Prodr. 459. Habitat in Nova Hollandia tropica. (R. Brown, Ferd. Bauer.) V. 60.

\section{Selagineae.}

3727. Hebenstreitia robusta $E$. Meyer Comm. 246. - Ha bitat in Capite bonae spei. (Drége.) VIII. 76.

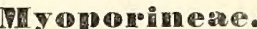

3733. Myoporum obscurum Endlicher Prodr. Flor. Norf. n.110. - Habitat in insula Norfolk. (Ferd.Bauer.) VI. 65.

3734. Pholidia scoparia R. Brown Prodr. 517. - Habitat in Nova Hollandia australi. (R. Brown, Ferd. Bauer, A. Cunningham.) VI. 66.

3736. Stenochilus glaber R. Brown Prodr. 517, - Habitat in Nova follandia australi. (R. Brown, Ferd Bauer.) VIII. 92.

\section{Comvolvulacede.}

3800. Polymeria calycina $\boldsymbol{R}$. Brown Prodr. 488. - Ha. bitat in Nova Hollandia orientali et tropica. ( $R$. Brown, Ferd. Bauer.) VI. 67.

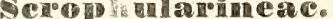

3902. Anthocercis littorea Labillard. Nov, Holl. H. 19 t. 158. R. Brown Prodr. 448, - Habitat in Nova Hollandia australi. (R. Brown, Ferd. Bauer, Hügel.) VI. 68 .

3906. Duboisia myoporoides R. Brown Prodr. 448. Habitat in Nova Hollandia orientali. (R. Brown, Ferd. Bauer, Sieber, Hügel, A. Cunningh.) VII. 77.

3981. Mazus Pumilio R. Brown Prodr. 439. - Habitat in van Diemens - Land. (R. Brown.) IX. 102

8938. Morgania pubescens R. Brown Prodr. 441. Habitat in Nova Hollandia tropica, $(\boldsymbol{R}$, Brown, Ferd. Bauer.) IX. 103.

\$9/4. Anticharis arabica Endlicher Nov. Stirp. Mus. Vin dob. Dec. $n$. 28. Capraria arabica Steud. et Hochst. msc. - Habitat in Arabia felici. (Fischer et Schimper.) VIII. 98.

3960. Buchnera urticifolia R. Brown Prodr. 437. Habitat in Nova Hollandia tropica. (R. Brown, Ferd. Bauer.) VII. 78.

4025. Haberlea rhodopensis Frivaldsky in Act. soc. Hung. 1835. p. 249. $t$. 1. - Habitat in Rumeliae montibus. (Frivaldsky.) VI. 69.

\section{andinaceac.}

4022. Nelsonia campestris $R$. Brown Prodr. 481. - Ha bitat in Nova Hollandia tropica. (R. Brown, Ferd. Bauer.) VII. 79.

4047. Ruellia bracteata $\boldsymbol{R}$. Brown Prodr. 479. - Habi tat in Nova Hollandia tropica. (R. Brown, Ferd. Bauer.) III. 104.

4072/1. Russeggera collina Endlicher Nov. stirp. Mus Vindob. Dec. n, 46. - Habitat in collibus Gebbel Accara, juxta fluviom Tumad Africae. (Russegger.) VIII. 94.

4097. Hypoëstes floribunda $\boldsymbol{R}$. Brown Prodr. 474. Habitat in Nova Hollandia tropica, (R. Brown, $F$ Bauer.) IX. 105.

\section{Sestmeac.}

4105. Sesamum indicum Linn. Spec. 884. Sims Bot Mag. $t$. 1788. - Habitat in India orientali et in insula Timor. (Ferd. Bauer.) VI. 70.

4106. Calampelis scabra Ion in Sweet Fl Gard. II t. 30. Eccremocarpus scaber Ruiz et Pavon Syst. 157. Lindley in Bot. Reg. t. 939. - Habitat in Chili. VIII. 95.

4112. Argylia radiata Don in Edinb. n. philosoph. Journ. 1823 n. 8. p. 260. Bignonia radiata Linn. Spec. 871 - Habitat in Chili. (Bridges.) VI. 71

\section{G edratinese}

4181. Josephinia grandiflora $\boldsymbol{R}$. Brown Prodr. 520. Habitat in Nova Hollandia tropica, ( $\boldsymbol{R}$. Brown Ferd. Bauer.) IV. 106.

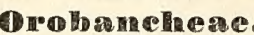

4182. Epiphegus americanus Nuttall Gen. Bor Amer. 549. Epiphegus virginianus Barton Flor Philad. II. 50. Mylanche virginiana Wallroth Diàsc. 76. Orobanche virginiana Linn. Spec. 882. - Ha bitat in America boreali ad radices Fagi. VII. 80.

4184. Conopholis americana Wallroth Diascev. 78. Oro banche americana Linn. Suppl. 88, - Habitat in Carolina, nec non in Mexico.(Andrieux.) VII. 81.

4189. Anoplanthus uniflorus Endl. msc. Orobanche uniflora Linn. Spec. 882. - Habitat in America boreali. VI. 72

4191. Hyobanche sanguinea Thunberg in Linn. Mant. 263. - Habitat in Capite bonae spei. (Drége) VII. 82 .

\section{Samotaceac.}

4240. Achras costata Endl. Flor. Norf. n. 96. - Habitat in insula Norfolk. (Ferd. Bauer, Hügel.) In Nova Zeelandia (A. Cunningham.) VIII. 83 . 
Colnumelliaceae.

4264. Columellia oblonga Ruiz et Pav. Flor. Peruv, 1. 28. $t$, 12. $f, a_{\circ}$ - Habitat in Peruvia. (Matthews.) VII. 84 .

\section{Saxifragaceac.}

4629. Eremosyne pectinata Endl. in Enum. Plant. Hügel. 53. - Habitat in Novae Hollandiae Colonia Swan-River. (Hügel.) X. 112.

4680. Aphanopetalum resinosum Endl. Nov. Stirp. Mus. Vindob. Dec. n. 42. - Habitat in Nova Hollandia orientali extratropica. (Ferd.Bauer, A. Cunning. ham, Hügel.) VIII. 96.

\section{Housseaceae.}

4680. Roussea simplex Smith Ic. inedit. I. 6. t. 6. DC. Prodr. VII. 522. - Habitat in insulae Mauritii sylvis umbrosis. (Sieber, Bojer.) IX. 107.

\section{Droseraceae.}

5035. Byblis liniflora Salisbury Parad t, 95, DC. Prodr. I. 319. - Habitat in Nova Hollandia, (Ferd, Bauer.) X. 113.

\section{Yiola}

5049. Hymenanthera latifolia Endl. Flor. Norf. n. 12\%. Habitat in insula Norfolk. (Ferd. Bauer, Hügel.) IX. 108.

\section{IPassi Moreae.}

5103. Modecca australis R. Brown ex DC. Prodr. III. 337. - Habitat in Novae Hollandiae borealis sinu Carpentaria, (R. Brown.) X, 114,115.

\section{Cuenrbitaceae.}

5127. Zehneria Baueriana Endl. Flor. Norf. n. 126. Habitat in insula Norfolk. (Ferd. Bauer, Hügel.) X. 116.117.

\section{IBittueriacese.}

5347. Xeropetalum multiflorum Endl. Nov. Stirp. Mus. Vind. Dec. n. 43. - Habitat in Shangul et Camamil Africae. (Theodorus Kotschy.) X. 118.

\section{Sapiuallaceac.}

5628. Plösslea floribunda Endl. Nov. Stirp. Mus, Vind. Dec, n. 47. - Habitat in Fazzoglou Africae tropicae. (Theodorus Kotschy.) X. 119. 120.

\section{Ir hammeae.}

5754. Penmantia corymbosa Forster Prodr. n. 379. Endl Flor. Norf. n. 140. - Crescit in insula Norfolk (Forster,-Ferd, Bauer.) X. 121.

\section{Euplin miaceae.}

5811. Baloghia lucida Endl. Flor. Norf. n. 143, - Habitat in insula Norfolk. (Ferd. Bauer, Hügrel.) X. 122. 123.

5812. Ricinocarpus pinifolins Desfont. in Mem. Mus. III. 459. t. 22. - Habitat in Nova Hollandia. (Ferd. Bauer, Sieber.) X. 124.

\section{Leguminthase.}

6607. Kotschya africana Endl. Nov. Stirp. Mus. Vind. Dec. n. 6. - Habitat in insula fluvii Tumad, in confinibus Shangul et Camamil Africae borealis. (Theodorus Kotschy.) X. 125 


\section{Index tabulamim.}

I. 1. 357. Ascröe pentactina Endl.

2. 882. Coelachne pulchella $\boldsymbol{R} . B r$.

3. 1072. Anguillaria dioica $R$. $B r$.

4. 1062. Eustrephus latifolius $R$. $B r$.

5. 1544. Gastrodia sesamoides $\boldsymbol{R}$. Br.

6. 1592. Eriochilus autumnalis $R$. $B r$

7. 1596. Lyperanthus nigricans $R$. $B r$.

8. 1598. Caleya major $R$. $B r$.

9. 1707. Gymnostachys anceps $R, B r$.

10. 2022. Doryphora Sassafras Endl.

11. 2098. Pimelea (Thecanthes) punicea R.Br.

12. 2131. Symphyonema paludosum $\boldsymbol{R}$. $B r$.

II. 13. 1025. Xyris (Pomatoxyris) operculata Lab.

14. 1579. Cryptostylis longifolia $R$. Br.

15. 1588. Microtis parviflora $R, B r$.

16. 1589. Acianthus fornicatus $R$. $B r$.

17. 1580. Calochilus paludosus $R$. $B r$.

18. 1597. Corysanthes (Steleocorys) unguiculata $\boldsymbol{R} . \boldsymbol{B r}$.

19. 1894. Putranjiva Roxburghii Wall.

20. 1967. Centrostachys aquatica Wallr.

21. 2019. Ruizia fragrans Pav.

22. 2105. Wikströmia australis Endl.

23. 2133. Stirlingia anethifolia Endl.

24. 2144. Hackea acicularis $R$. $B r$.

III. 25. 963. Diplacrum caricinum $\boldsymbol{R}, \boldsymbol{B r}$.

26. 1046. Limnocharis Plumieri Rich.

27. 1104. Blandfordia nobilis Smith.

28. 1164. Arthropodium paniculatum $\boldsymbol{R}$. $\mathrm{Br}$

29. 1616. Thelychiton argyropus Endl.

30. 2012. Pisonia grandis $R$. Br.

31. 2132. Conospermum (Euconospermum) ericifolium Smith.

32. 2131. Synaphaea dilatata $R, B r$.

33. 2143. Grevillea (Lissostylis) riparia $R, B r$

34. 2213. Hoplophyllum spinosum $D C$.

35. 2374. Fresenia leptophylla $D C$

36. 2629. Madaria corymbosa $D C$.

IV. 37. 1045. Hydrocleia Commersonii Rich.

38. 1059. Calectasia cyanea $\boldsymbol{R}$. $B r$.

39. 1184. Smilax glyeyphylla $\boldsymbol{R}$. $\boldsymbol{B} r$.

40. 1534. Bartholina peetinata Lindl.

41. 1594. Glossodia major $\boldsymbol{R} \cdot \boldsymbol{B r}$.

42. 1650. Ravenala madagascariensis Sorner.

43. 2068. Gyrocarpus sphenopterus $\boldsymbol{R} . \boldsymbol{B r}$.

44. 2066. Litsaea Baneri Endl.

45. 2075. Choretrum glomeratum $R$. Br.

46. 2132. Conospermum (Chilurus) teretifo lium R. Br.

47. 2146 Xylomelum pyriforme Smith.

V. 49. 1106. Centrolepis fascicularis Labill.

50. 1234. Patersonia glauca $\boldsymbol{R}$. $\boldsymbol{B r}$.

51. 1593. Caladenia carnea $R$. Br.

52. 2134. Franklandia fucifolia $\boldsymbol{H}, \boldsymbol{B r}$.

53. 3062. Heterotoma lobelioides Zucc
54. 3349. Olea (Gymnelaea) apetala Vahl.

55. 3350. Notelaea ovata $R$. $B r$.

56. 3362. Antonia pilosa Hook.

57. 3364 Logania (Euosma) floribunda $R$. Br.

58. 3364. \{Logania (Stomandra) pusilla $R$. Br.

59. 3378. Melodinus Baueri Endl.

60. 3516. Microstemma tuberosum $R . B r$.

VI. 61. 1153. Tricoryne elatior $R, B r$.

62. 1978. Deeringia celosioides $\boldsymbol{R}$. $B r$.

63. 3444. Hybanthera biglandulosa Endl.

64. 3456. Sarcostemma australe $R, B r$.

65. 3733. Myoporum obscurum Endl.

66. 3734. Pholidia scoparia $R$. Br.

67. 3800. Polymeria calycina $R$. $B r$.

68. 3902. Anthocercis littorea Labill.

69. 4025. Haberlea rhodopensis Frivalds.

70. 4105. Sesamum indicum Linn.

71. 4112. Argylia radiata Don.

72. 4189. Anoplanthus uniflorus Endl.

VII. 73. 1664/1. Cycnogeton Hügelii Endl.

74. 2075. Leptomeria (Oxymeria) acida $R$. Br.

75. 3426. Balfouria saligna $R$. $B r$.

76. 3727. Hebenstreitia robusta $E$, Mey.

77. 3906. Duboisia myoporoides $\boldsymbol{R}, \boldsymbol{B r}$.

78. 3960. Büchnera urticifolia $R$. $B r$.

79. 4032. Nelsonia campestris $\boldsymbol{R}$. $B r$.

80. 4182. Epiphegus americanus Nutt.

81. 4184. Conopholis americana Wallr.

82. 4191. Hyobanche sanguinea Thunb.

83. 4240. Achras costata Endl.

84. 4264 . Columellia oblonga Lindl.

VIII. 85. 695. Psilotum (Tmesipteris) Forsteri Endl. 86. 1884. Böhmeria australis Endl.

87. 1986. Polygonum (Helxine) australe $A$. Rich

88. 202. Wollastonia Forsteriana $D C$.

89. 3240. Hedyotis (Anotis) gentianoides Endl.

90. 3275. Cosmibuena ochracea Endl.

91. 3504/1. Fockea capensis Endl.

92. 3736 Stenochilus glaber $\boldsymbol{R}$. $\boldsymbol{B}$.

93. 3944. Anticharis Schimperi Endl.

94. 4072/1. Russeggera collina Endl.

95. 4106. Calampelis scabra Don.

96 4650. Aphanopetalum resinosum Endl.

IX. 97. 1169. Laxmannia gracilis $R . B r$.

98. 1253. Haemodorum spicatum $R$. Br.

99. 1369. Dendrobium teretifolium $R . B r$.

100. 1920/1. Didymanthus Roëi Endl.

101. 3363/1. Coelostylis loganioides Torr, et A,Gr.

102. 3931. Mazus pumilio $R . B r$.

103. 3938. Morgania pubescens $R$. $B r$.

104. 4047. Ruellia bracteata $R$. $B r$

105. 4097. Hypoëstes floribunda $\boldsymbol{R} . \boldsymbol{B r}$.

106. 4181. Josephinia grandiflora $R$. Br.

107. 4680. Roussea simplex Smith.

108. 5049. Hymenanthera latifolia Endl. 
X 109. 1259/1. Tribonanthes australis Endl.

110. 2130. Adenanthus terminalis $\boldsymbol{R}$. $\mathrm{Br}$.

111. 3105. Coprosma Baueri Endl.

112. 4629. Eremosyne pectinata Endl.

113. 5035. Byblis Liniflora Salisb.

114. 5103. Modecca australis $R$. Br. 115.

116. 5127 . Zehneria Baueriana Endl.
118. 5347. Xeropetalum multifloram Endl.

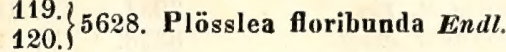

121. 5754. Pennantia corymbosa Forst.

122. $\}$

123. 5811. Baloghia lucida Endl.

124. 5812. Ricinocarpus pinifolius $D e s f$.

125. 6607. Kotschya africana Endl.

\section{Index alphabeticus.}

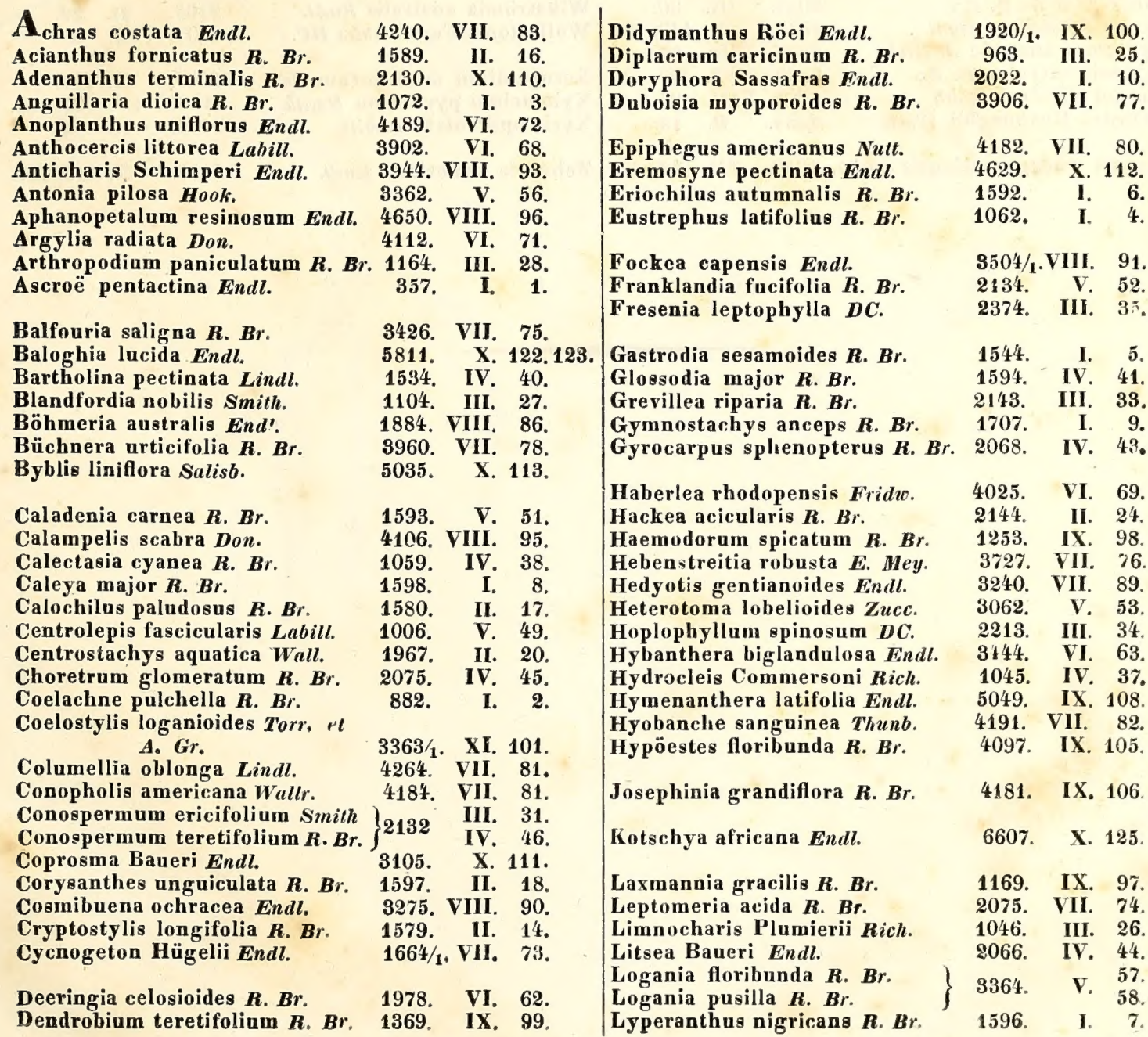




\section{XVI}

Madaria corymbosa $D C$.

Mazus Pumilio $\boldsymbol{R}$. $\boldsymbol{B}$ r.

Melodinus Baueri Endl. $\quad 3378 . \quad$ V. 59.

2629. III. 36 .

3931. IX. 102

Microstemma tuberosum $\boldsymbol{R} . \boldsymbol{B} r$. 3516 . V. 60 .

Microtis parviflora $\boldsymbol{R} . \boldsymbol{B r}$. 1588. II. 15.

Modecea australis $\boldsymbol{R}$. $\boldsymbol{B r}$.

Morgania pubescens $\boldsymbol{R} . \boldsymbol{B r}$.

$5103 . \quad X .114 .115$

Myoporum obscurum Endl.

3938. IX. 103.

3733. VI. 65 .

Nelsonia campestris $\boldsymbol{R} . \boldsymbol{B}$.

Notelaea ovata $\boldsymbol{R}$. B.

4032. VII. 79 .

$3350 . \quad$ V. 55.

Olea apetala $\mathrm{Vahl}$.

3349.

Patersonia glauca $\boldsymbol{R}$. $\mathrm{Br}$.

Pennantia corymbosa Forst.

Pholidia scoparia $\boldsymbol{R}$. Br.

Pimelea punicea $\boldsymbol{R}$. Br.

Pisonia grandis $\boldsymbol{R}$. Br.

Plösslea floribunda $E n d l$.

Polygonum australe $\boldsymbol{A}$. Rich.

Polymeria calycina $\boldsymbol{R}$. $\boldsymbol{B r}$.

Psilotum Forsteri Endl.

1234. V. 50

5754. X. 121.

3734. VI. 66

2098. I. 11

2012. III. 30 .

5628. X. 119

1986. VIII. 87.

3800 . VI. 67.

Putranjiva Roxburghii Wall.

695. VIII, 85 .

1894. II. 19 .

V. 54.

Ricinocarpus pinifolius Desf.

Roussea simplex Smith.

Ruellia bracteata $\boldsymbol{R}$. Br.

Ruizia fragrans $P a v$.

Russeggera collina Endl.

Sarcostemma australe $\boldsymbol{R}$. $\boldsymbol{B r}$.

Sesamum indicum Linn.

Smilax glycyphylla $\boldsymbol{R}$. $\boldsymbol{B r}$.

Stenochilus glaber $\boldsymbol{R}$. $\boldsymbol{B}$.

Stirlingia anethifolia Endl.

Symphyonema paludosum $\boldsymbol{R}$. $B r$.

Synaphea dilatata $\boldsymbol{R}$. $\boldsymbol{B}$ r.

Thelychiton argyropus Endl.

Tribonanthes australis Endl.

Tricoryne elatior $\boldsymbol{R} . \boldsymbol{B r}$.

Wikströmia australis Endl.

Wollastonia Forsteriana $D C$.

Xeropetalum multiflorum Endl.

Xylomelum pyriforme Smith.

Xyris operculata Labill.

Ravenala madagascariensis Smith. 1650. IV. 42. $\quad$ Zehneria Baueriana Endl. $\quad$ 5127. X. 116.117.

Ravenala madagascariensis Smith. 1650. IV. 42. Zehneria Baueriana Endl. $\quad$ 5127. X. 116.117.

5812. X. 124

4680. IX. 107

4047. IX. 104

2019. II. 21

4072/1. VIII. 94 .

3456. VI. 64

4105. VI. 70.

1184. IV. 39

3736. VIII. 92

2133. II. 23

2131. I. 12

2131. III. 32 .

1616. III. 29

1259/1. X. 109

1153. VI. 61.

2105. II. 22.

2502. VIII. 88

534\%. X. 118

2146. IV. 47.48

1025. II. 13.

Ravenala madagascariensis Smith. 1650. IV. 42. Zehneria Baueriana Endl. $\quad$ 5127. X. 116.117. 
Gasteromycetes.

Phalloidei./35\%.)

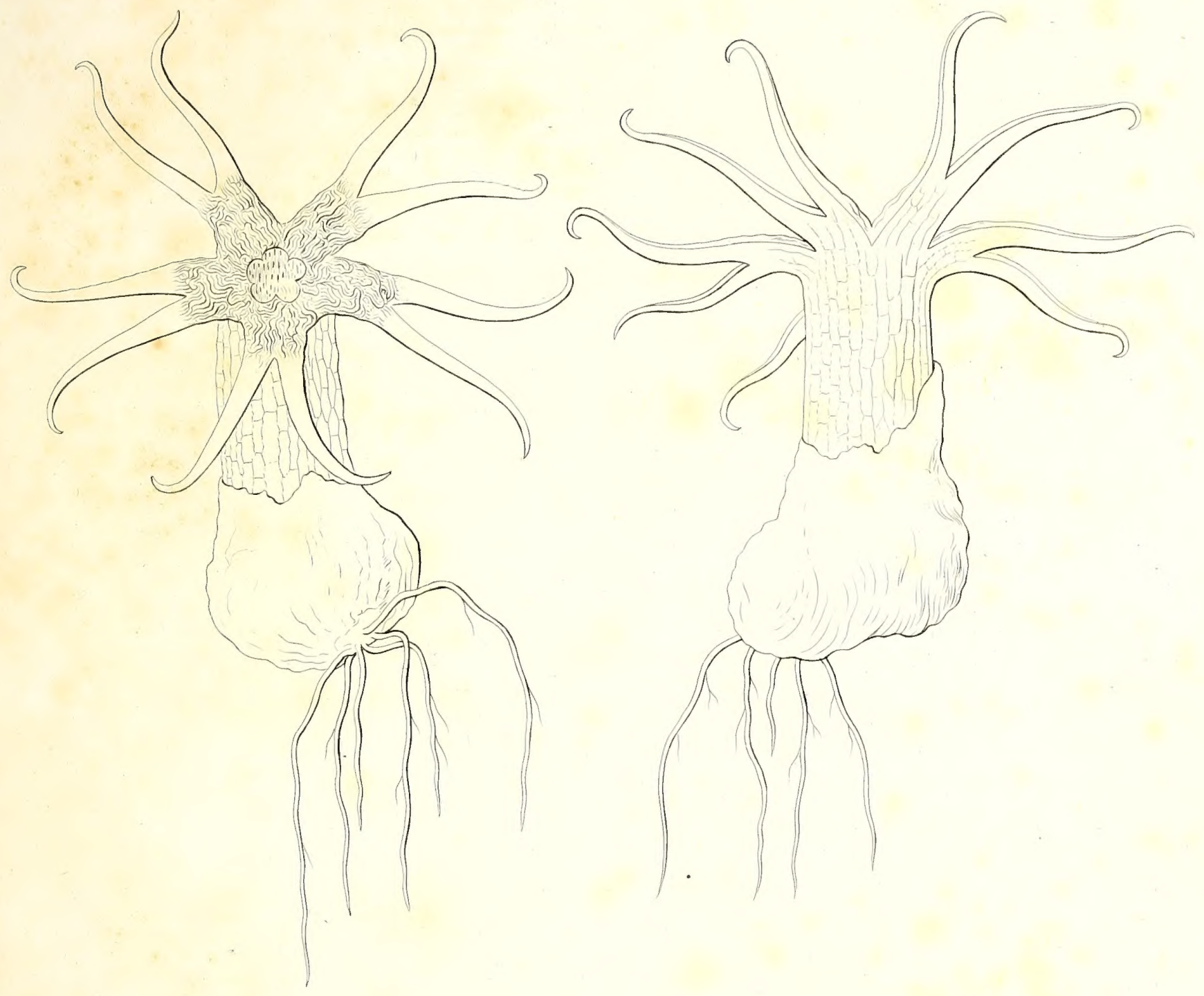

Hocrie pentactina $\check{\text {. }}$

Ferd. Saunes del.

Golhantso. (1.) 


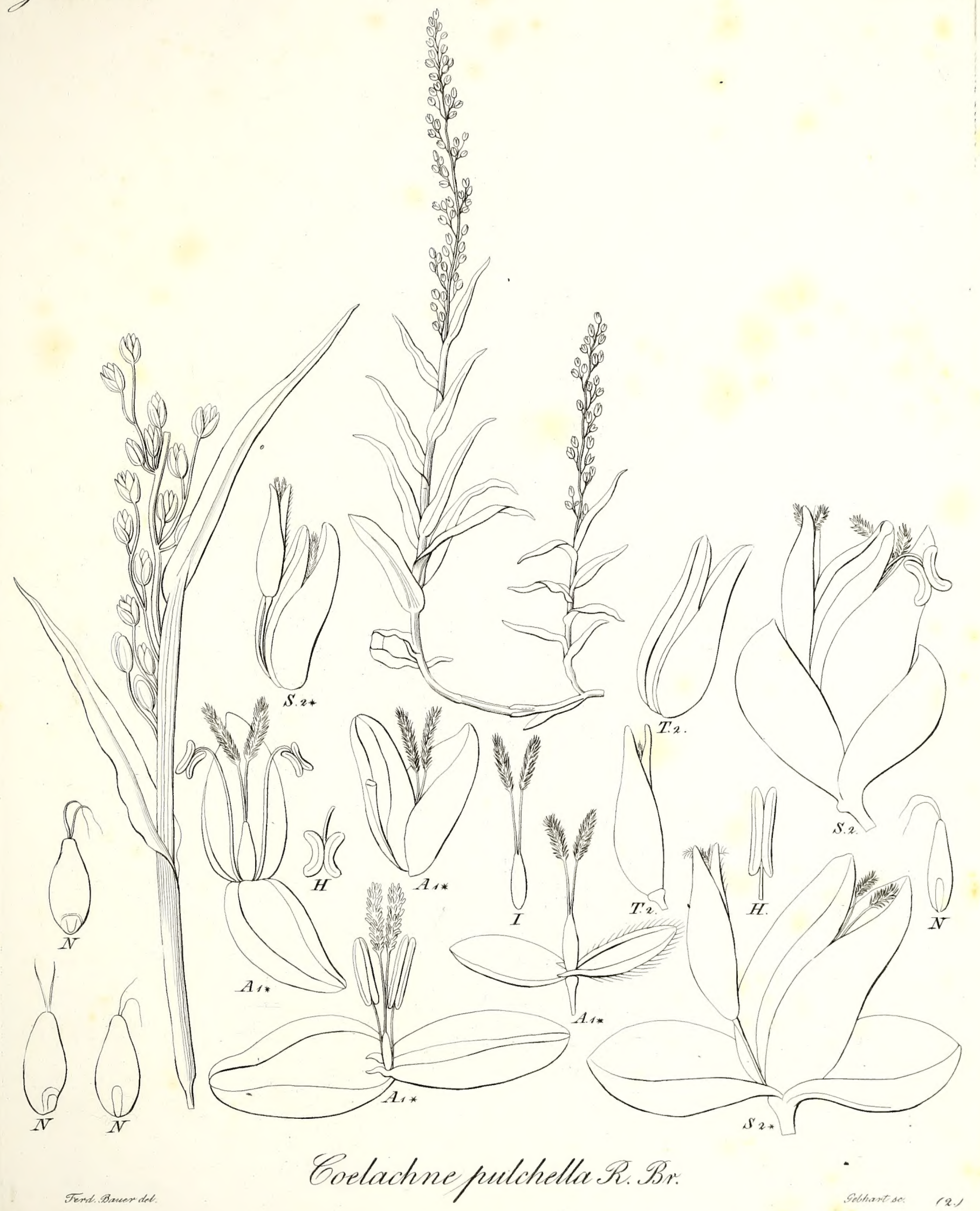



Nelanthacese.

Veratrea /royg/

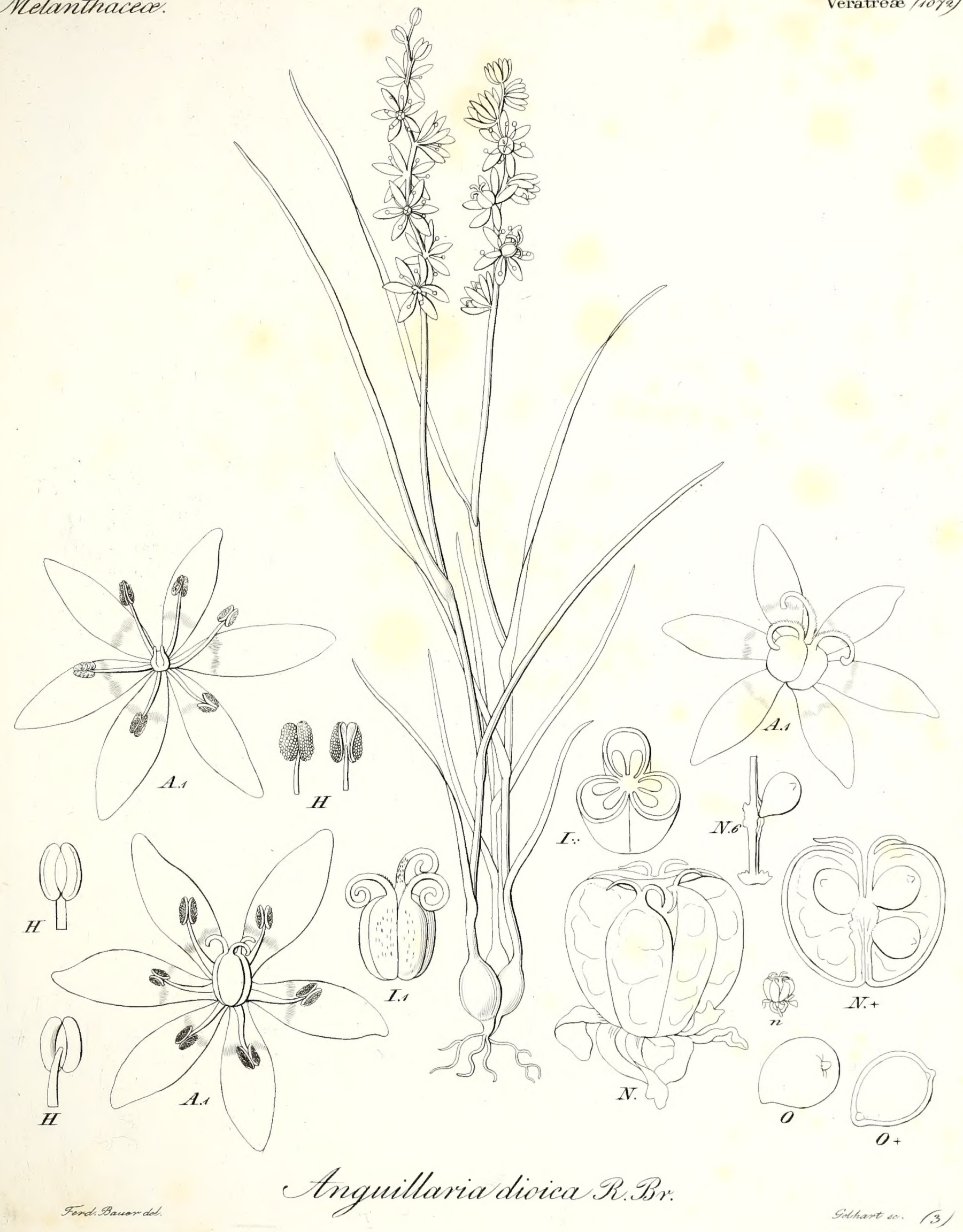





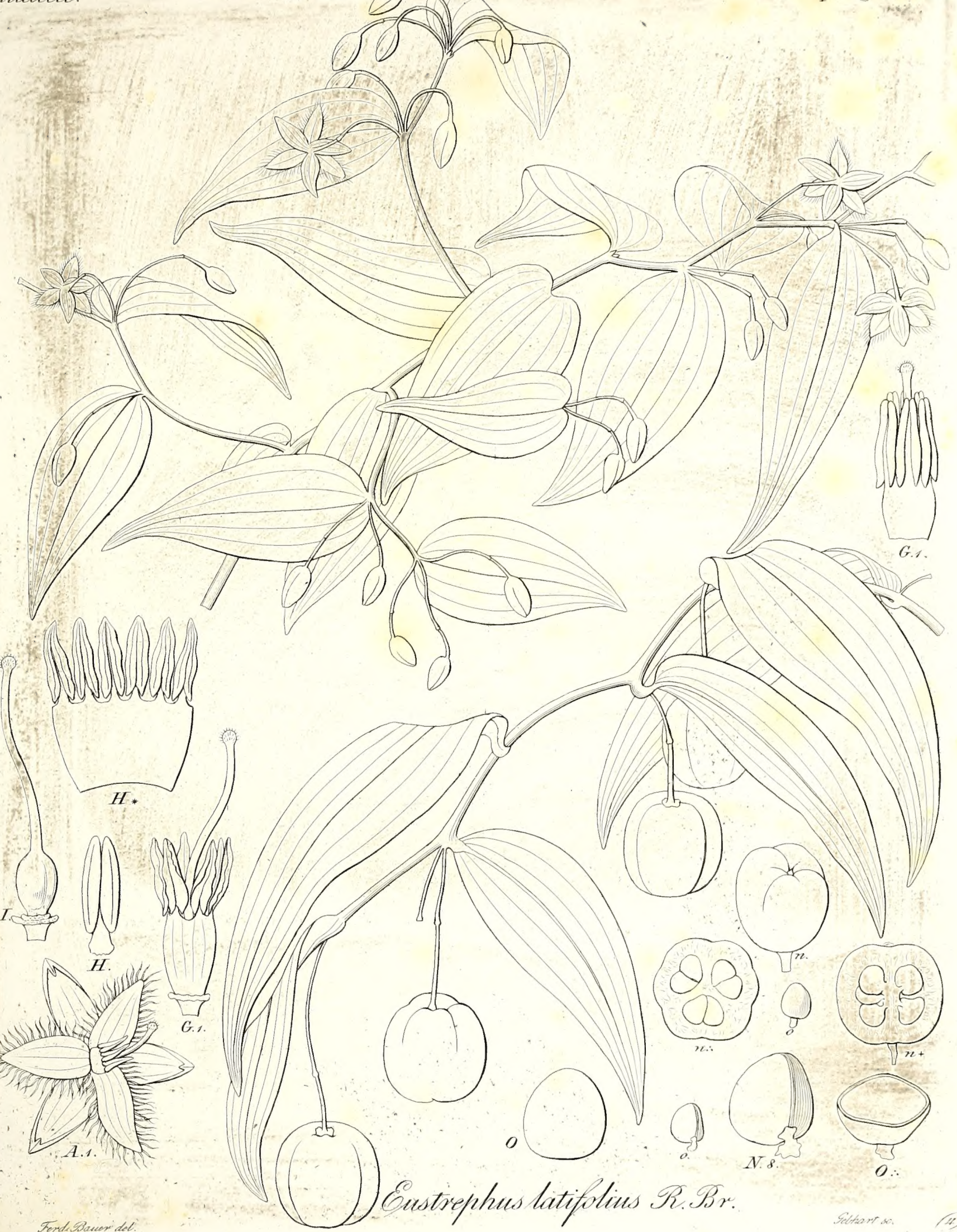




\section{Orchidea.}

Gastrodieas (154h.

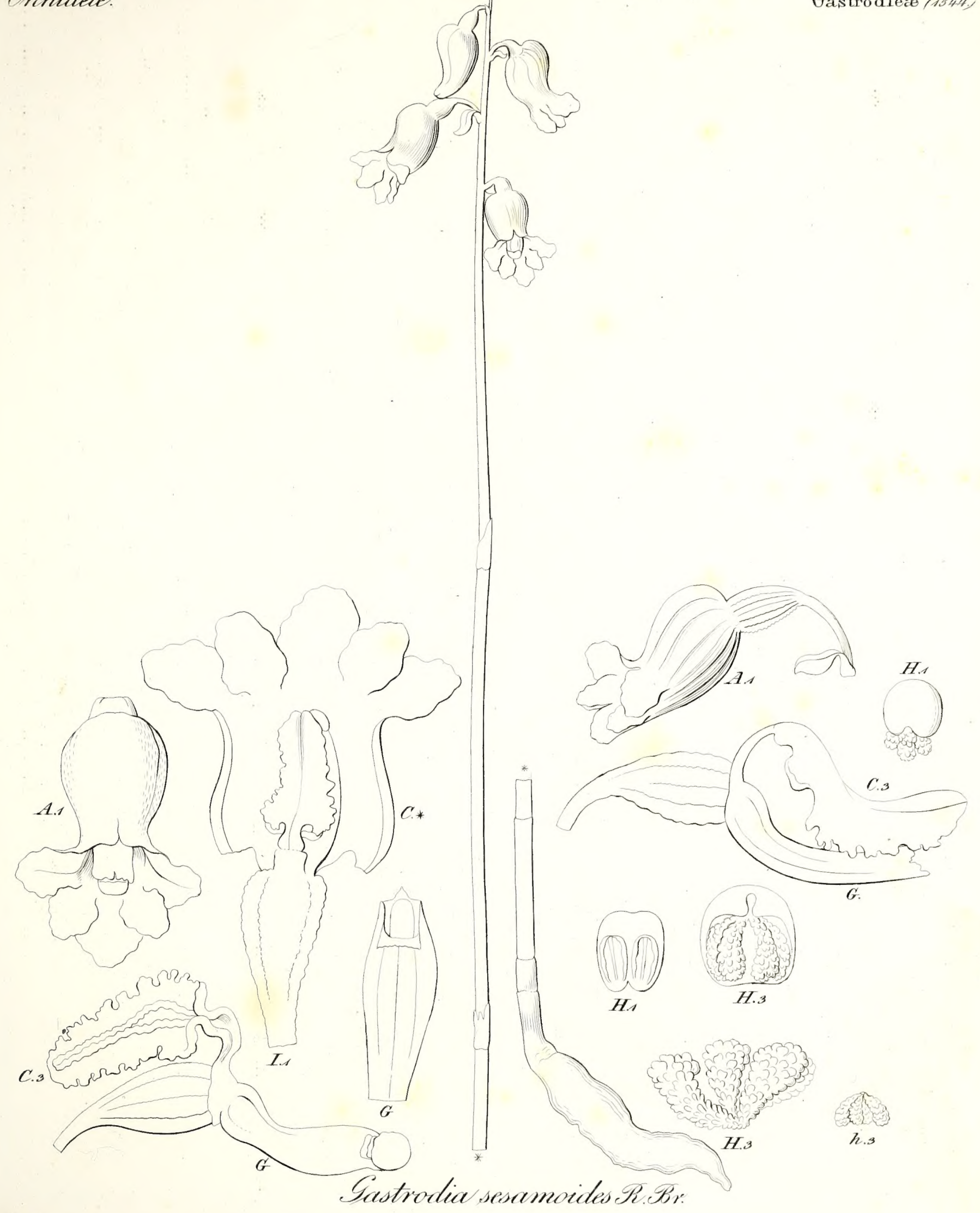




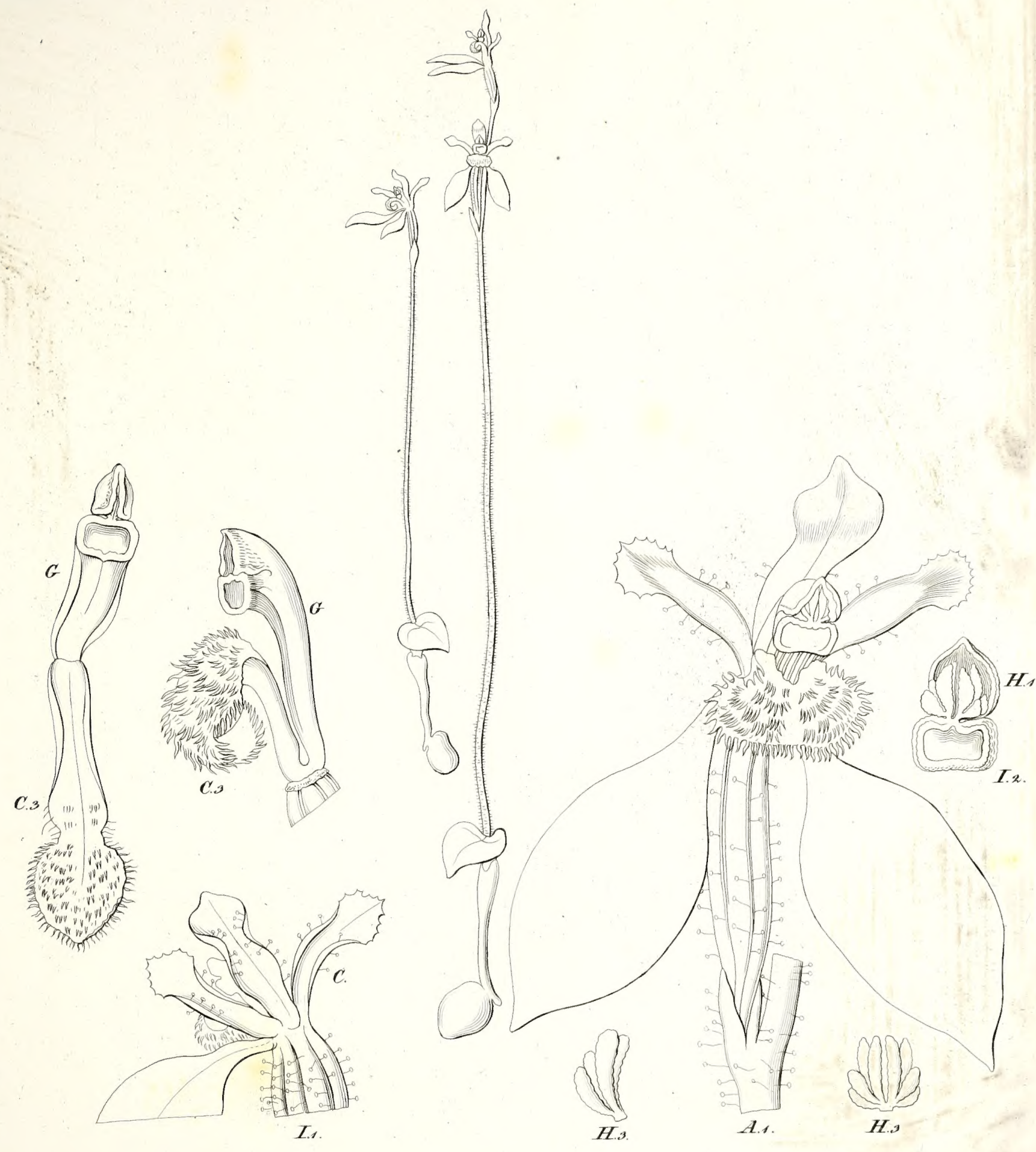

Griochilus autumnalis R. R $\mathscr{B}$. 


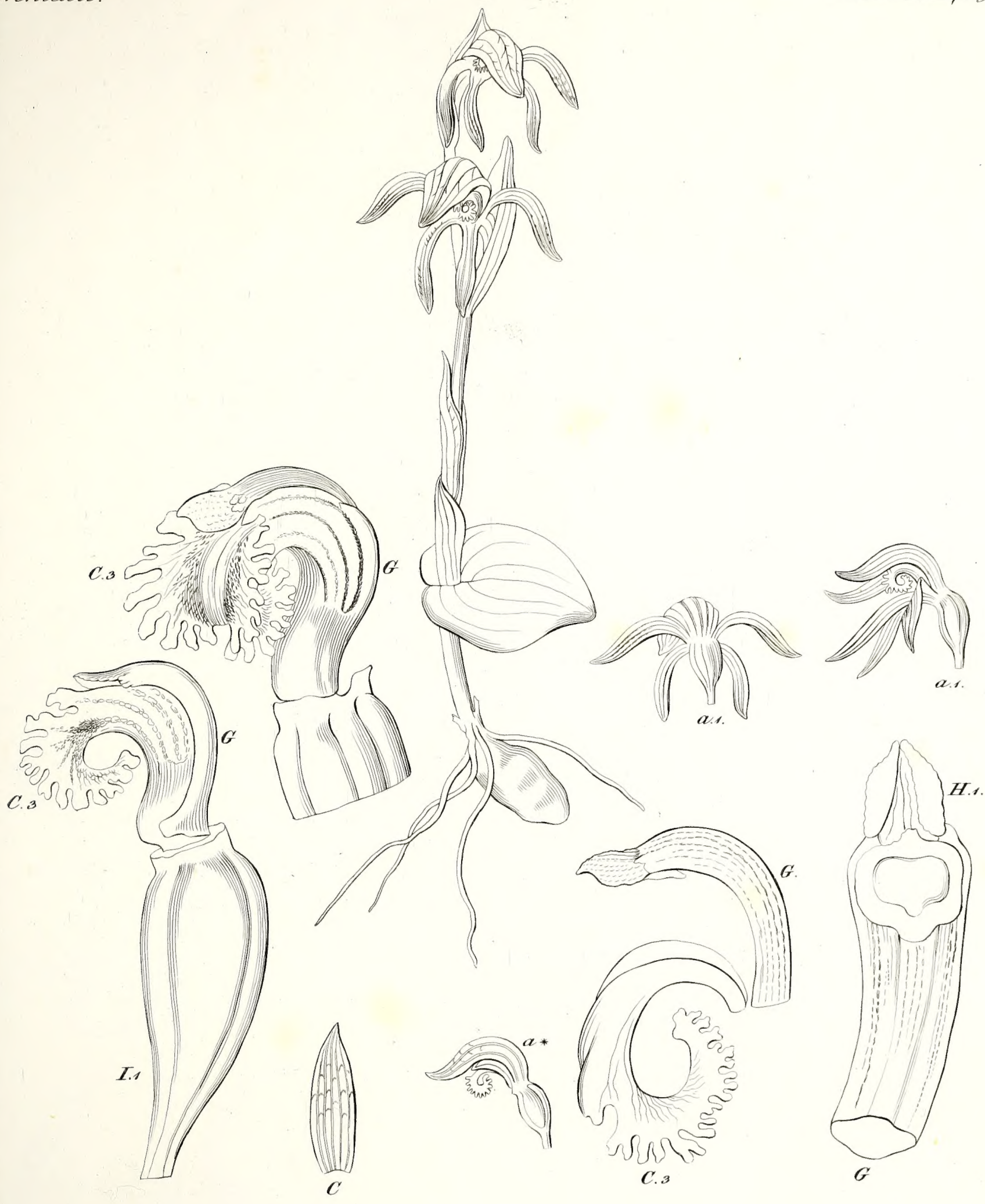



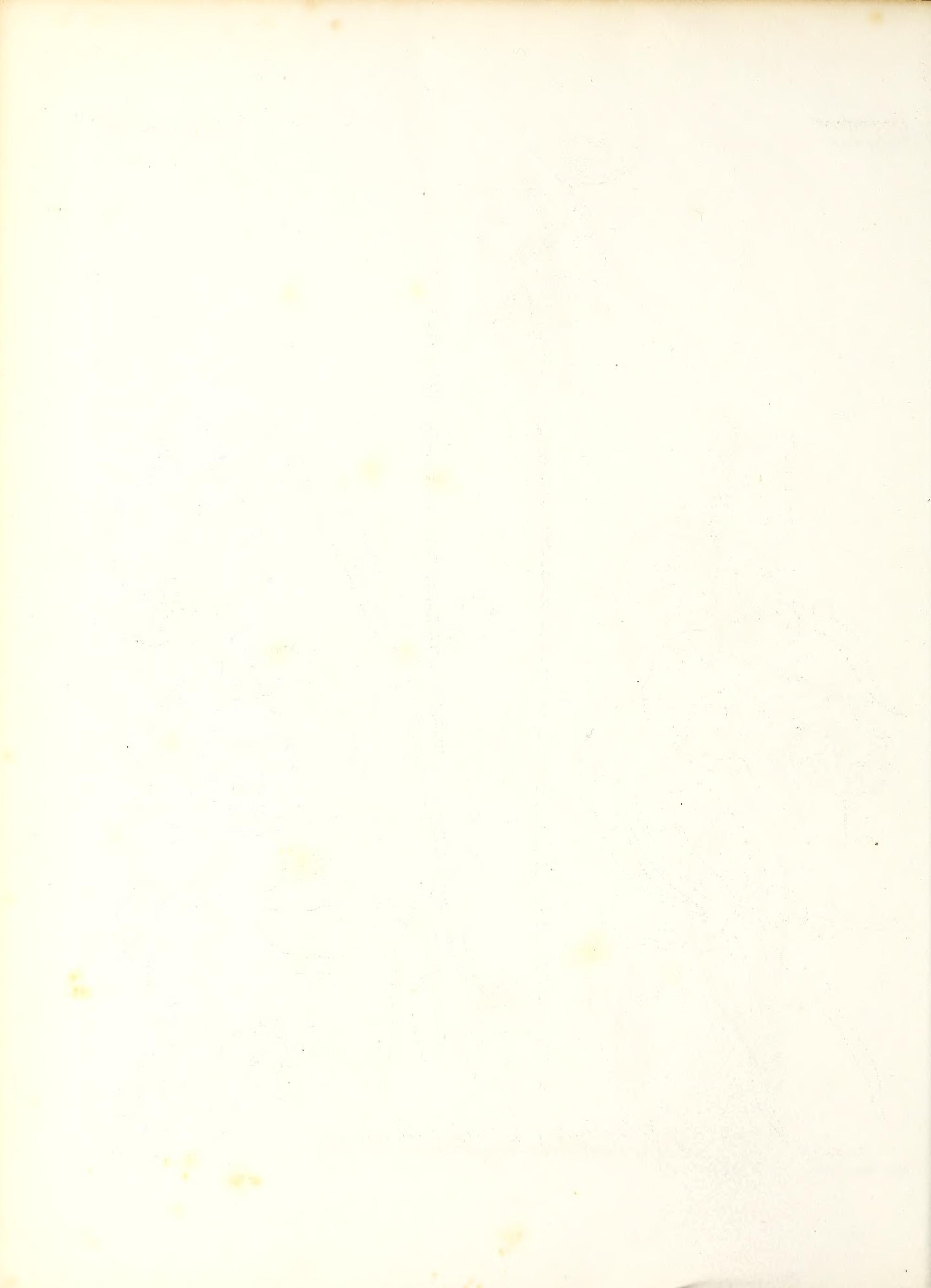
Orchidew.

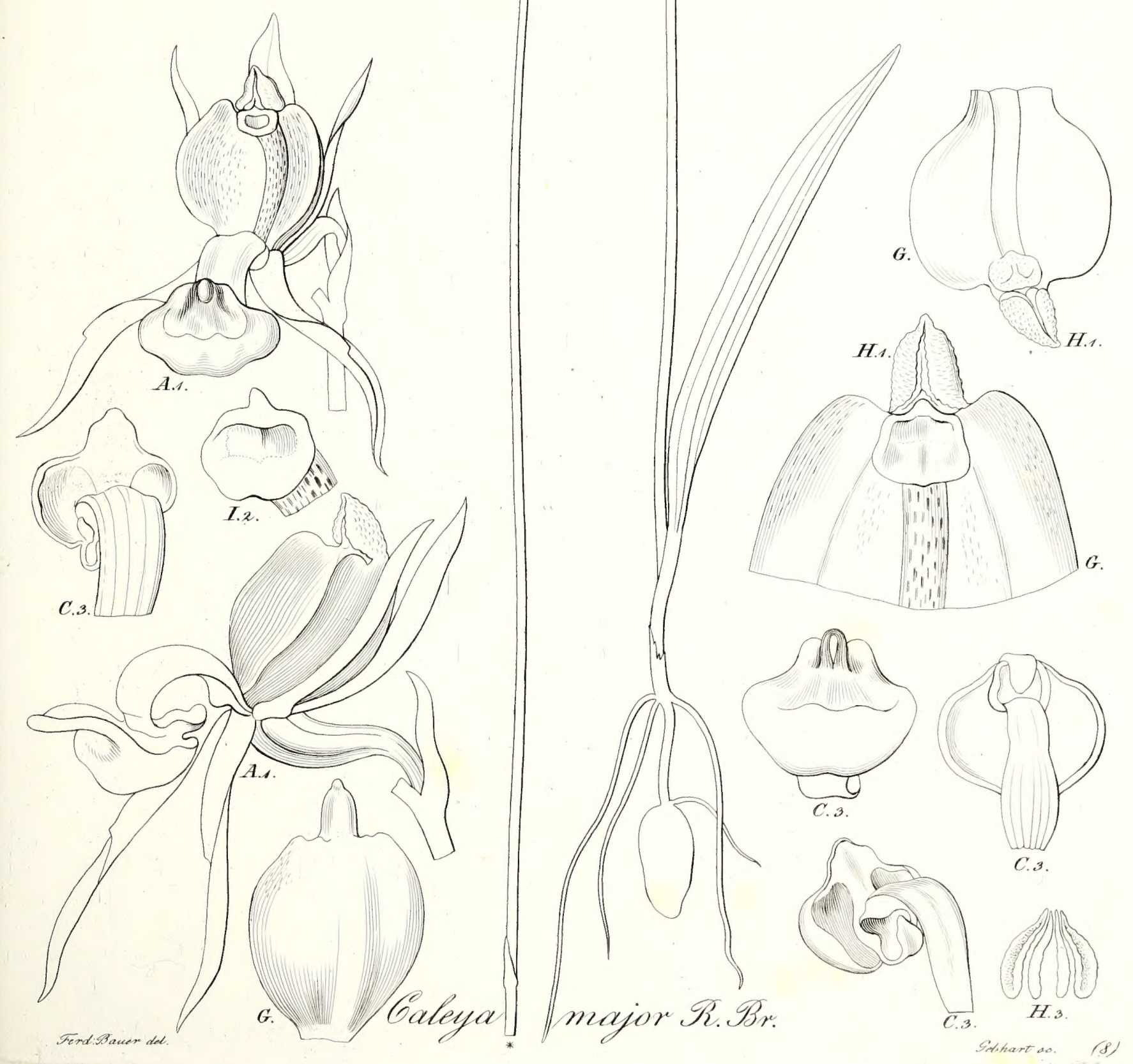



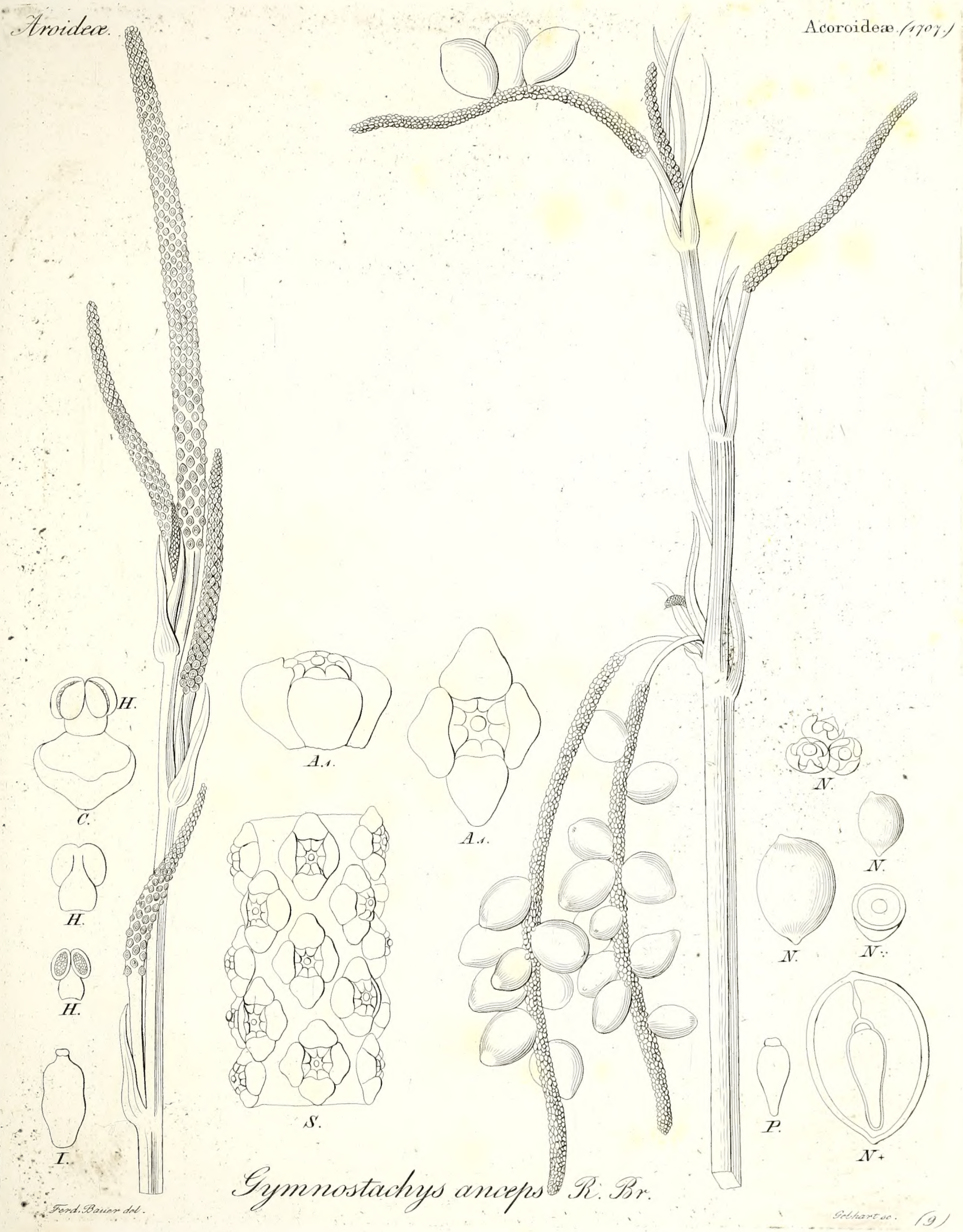



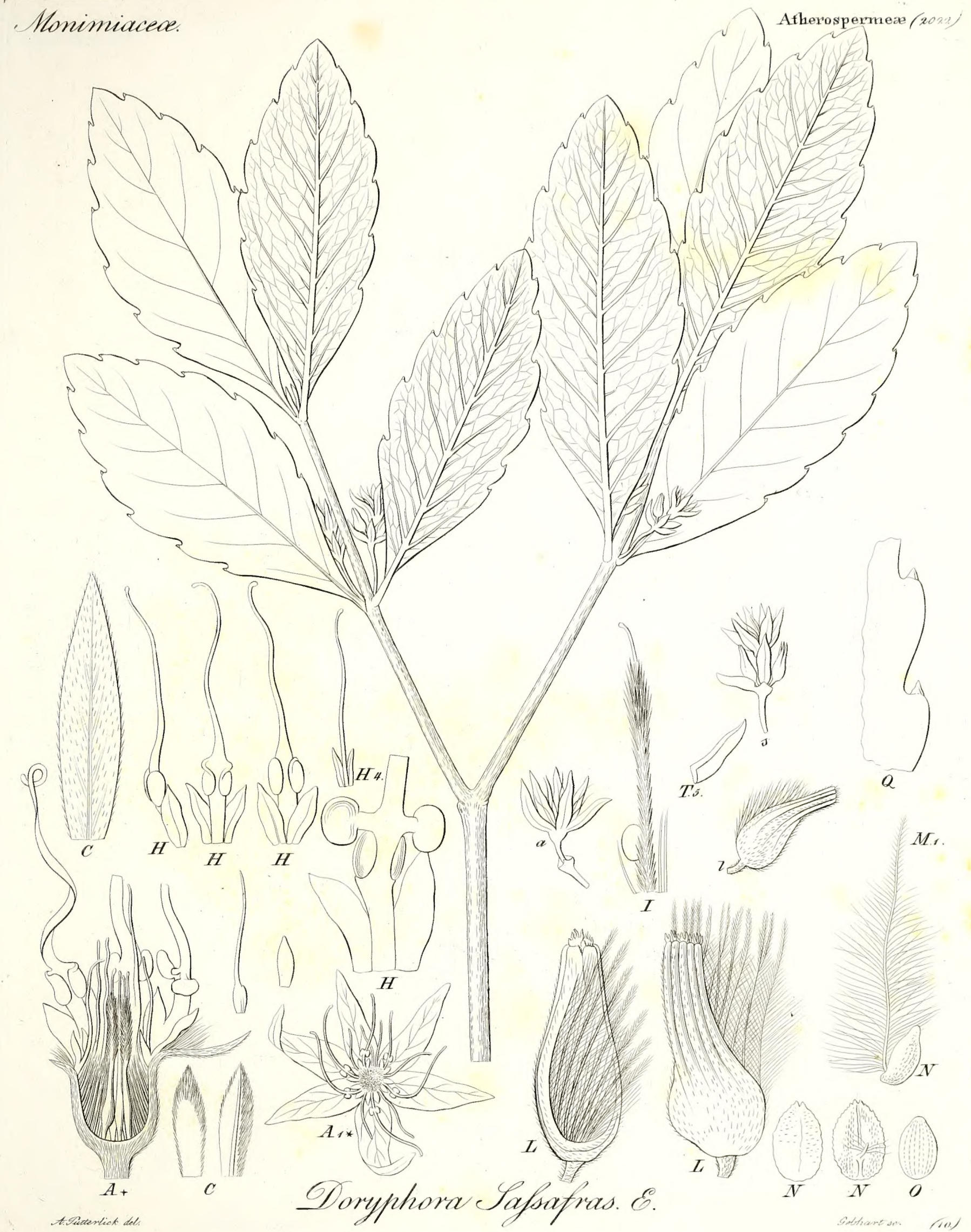


Exphnoidex.

(roges:a)
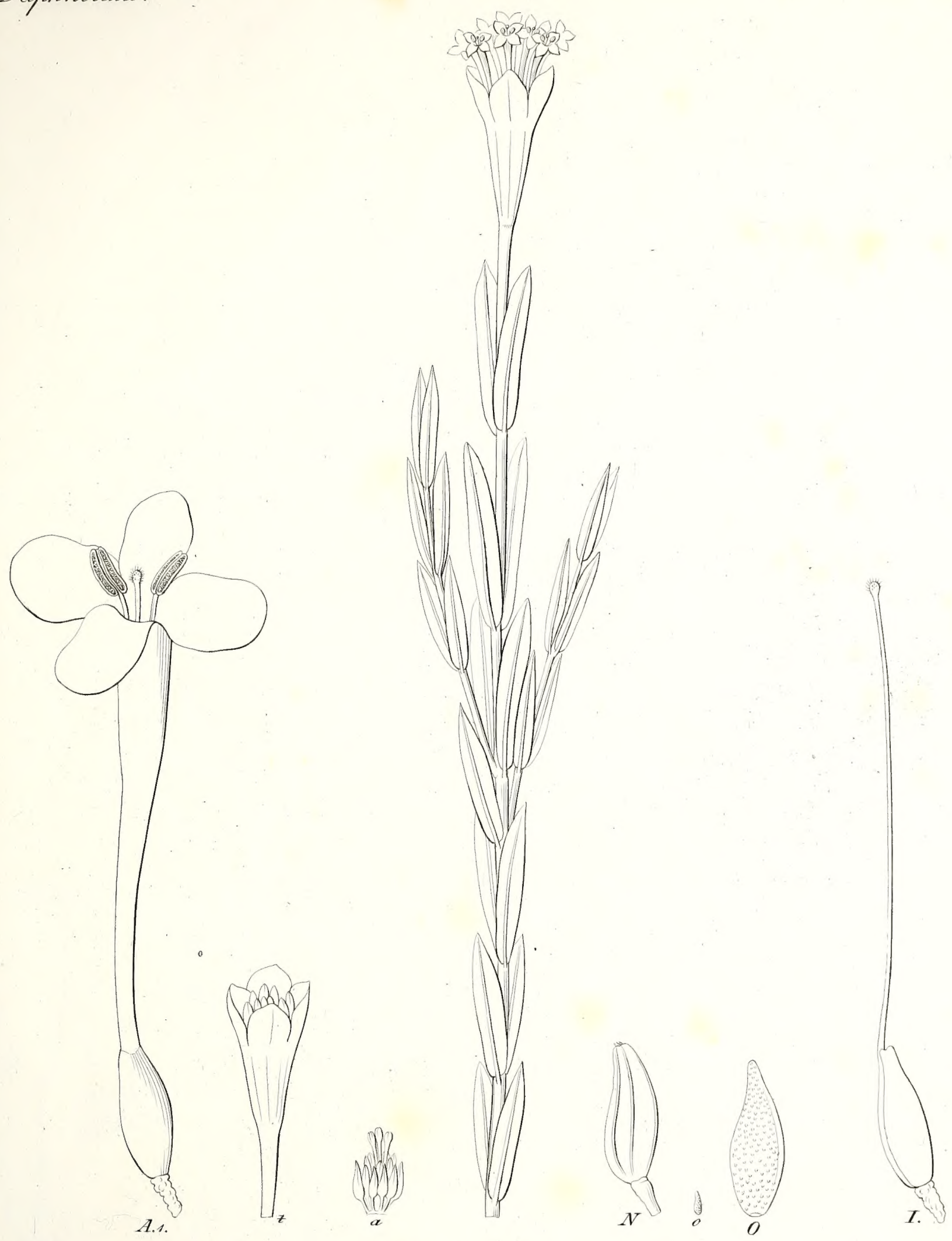

Pimelea/ Thecanthes/punicea R. Rr.

Ford. Bawer del.

Gidhare so. (11) 
Troteaceie.

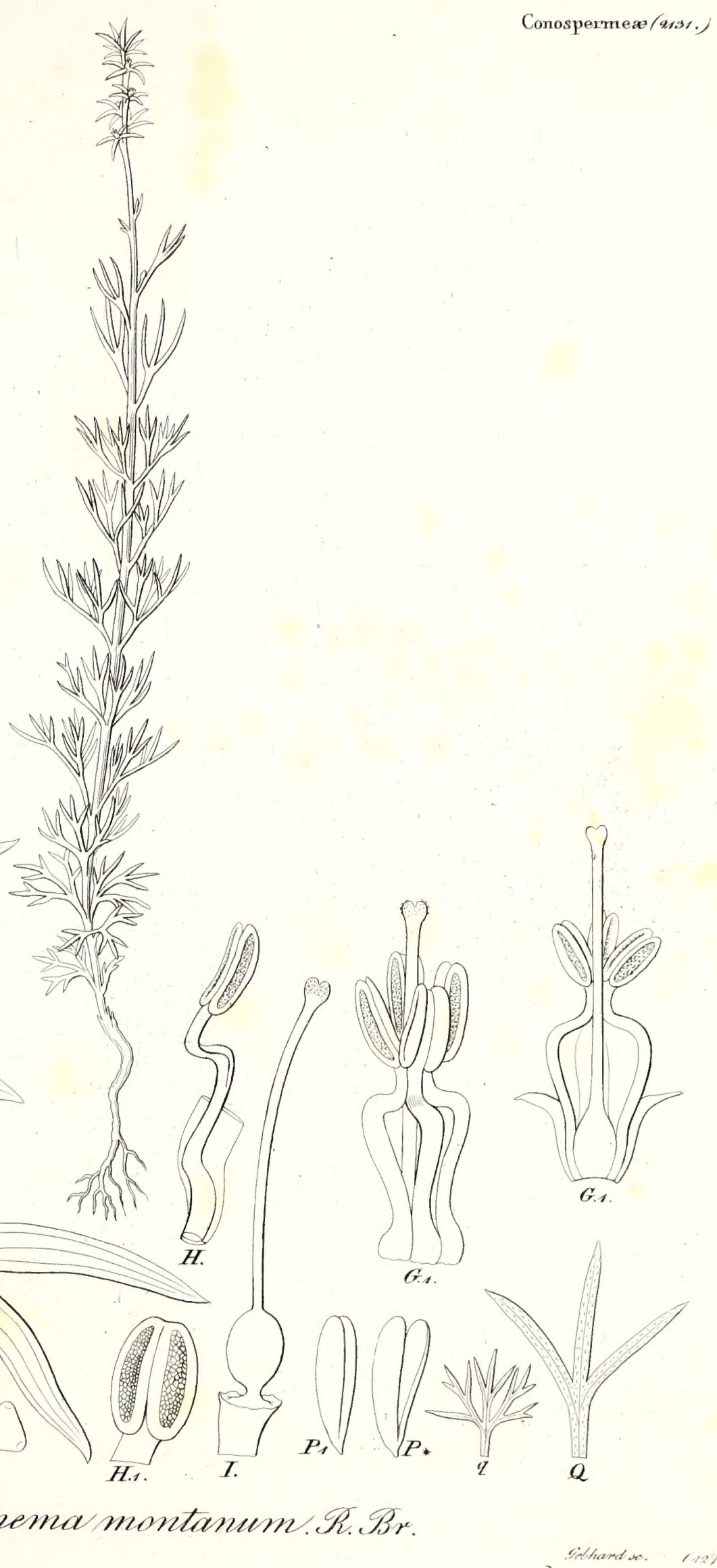


Bayridex.

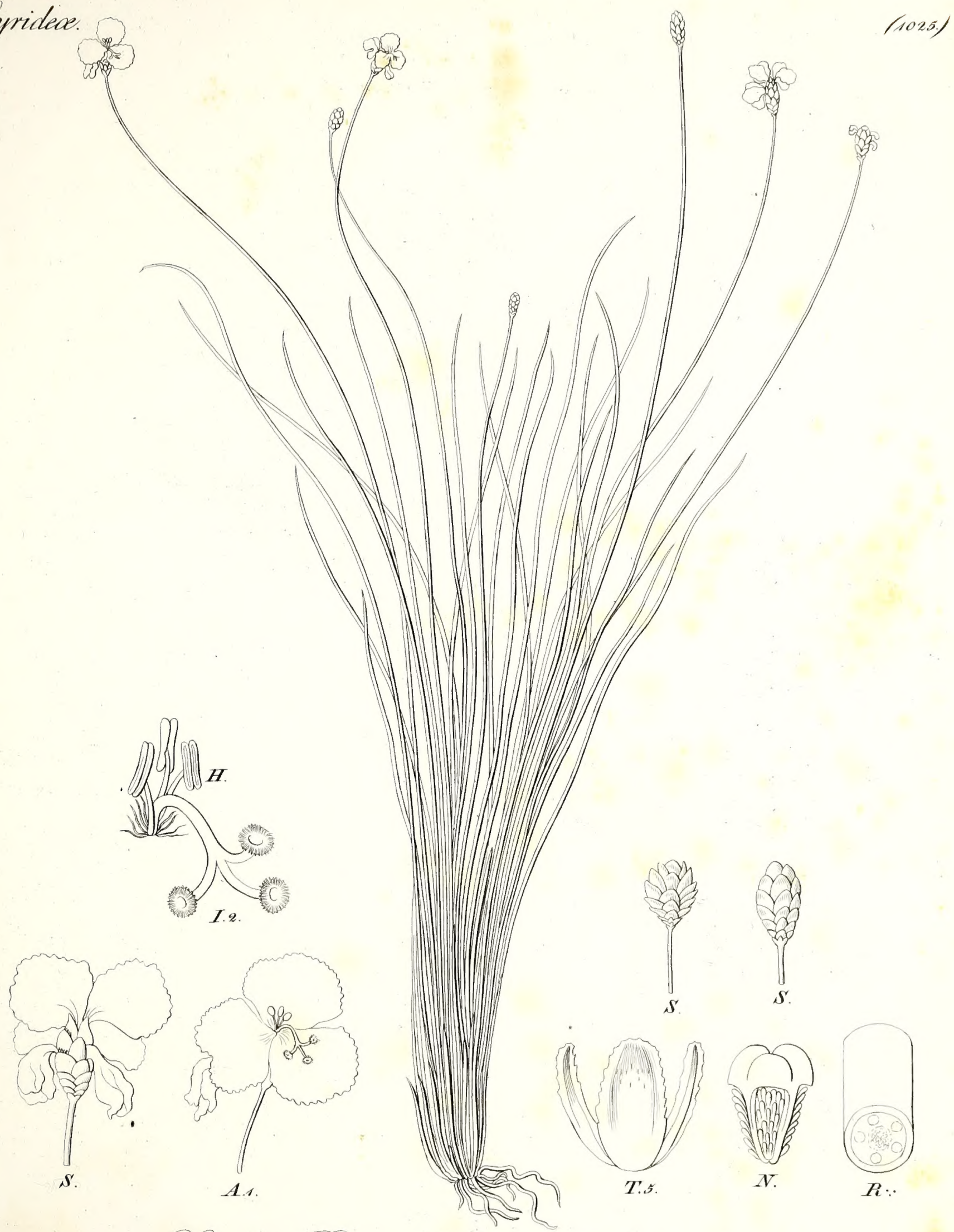

Pynis/Promatoxyris/operculata Lab. 

Orchidea.

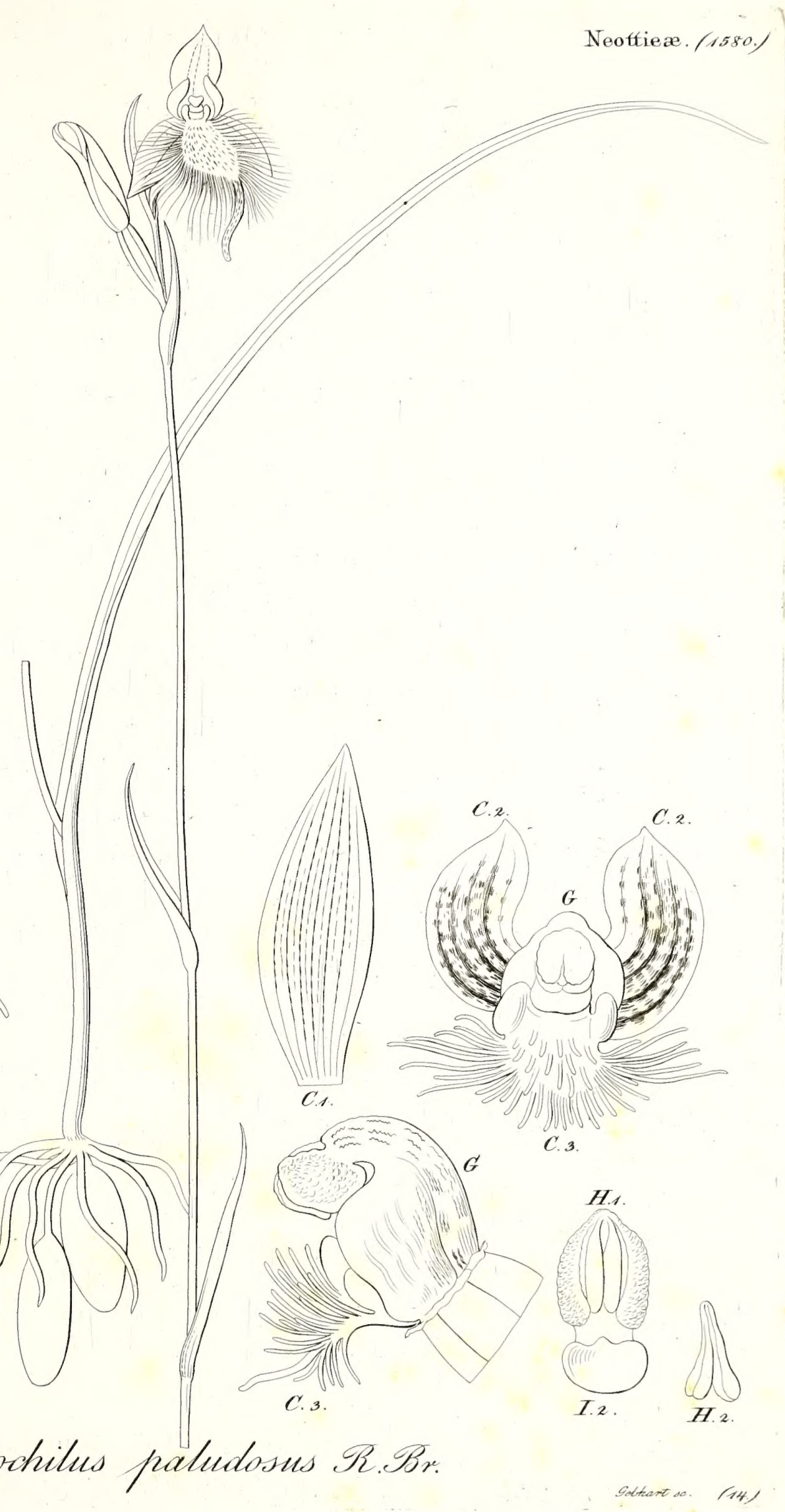


Orchidece.

Arethusere (1588)

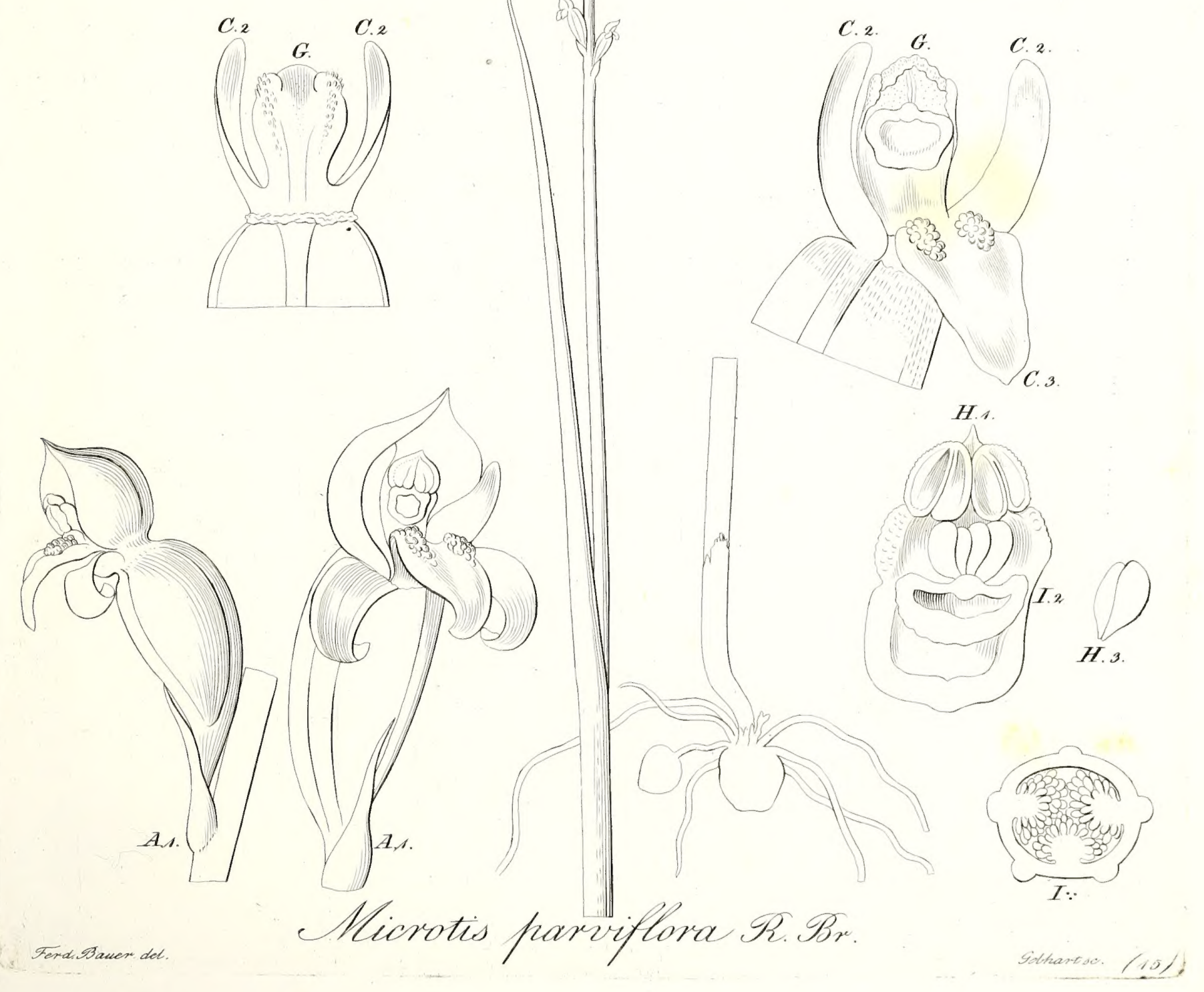





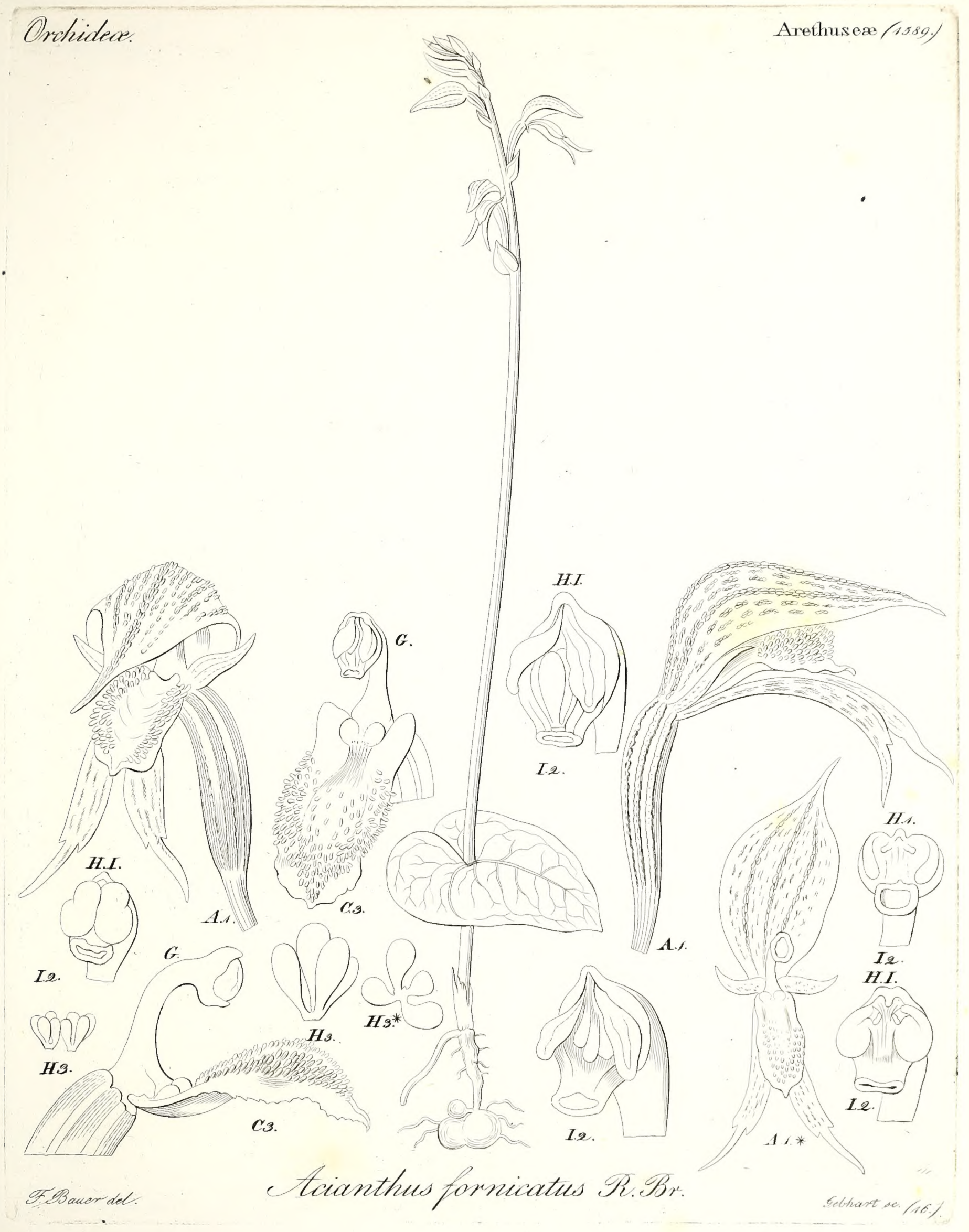





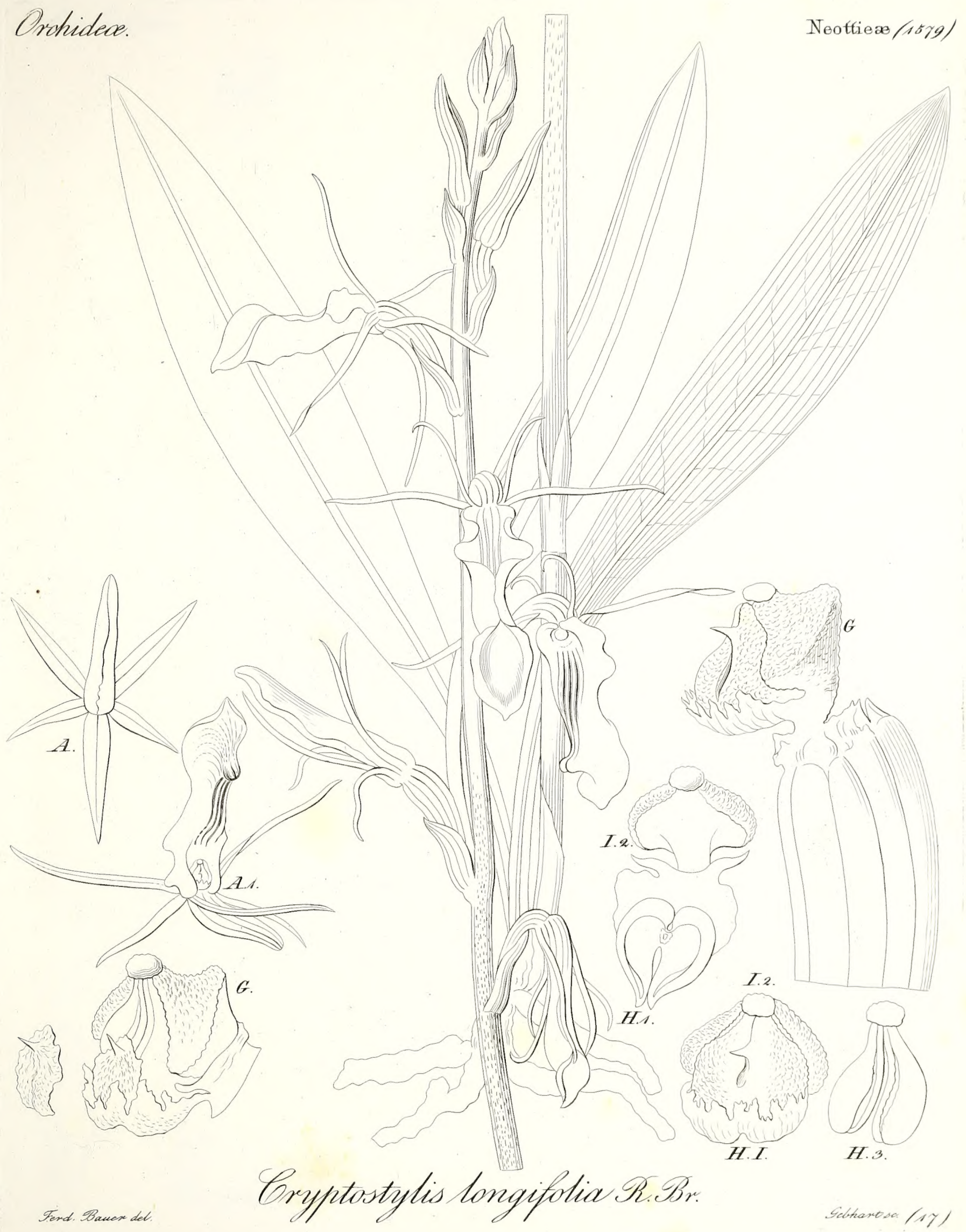





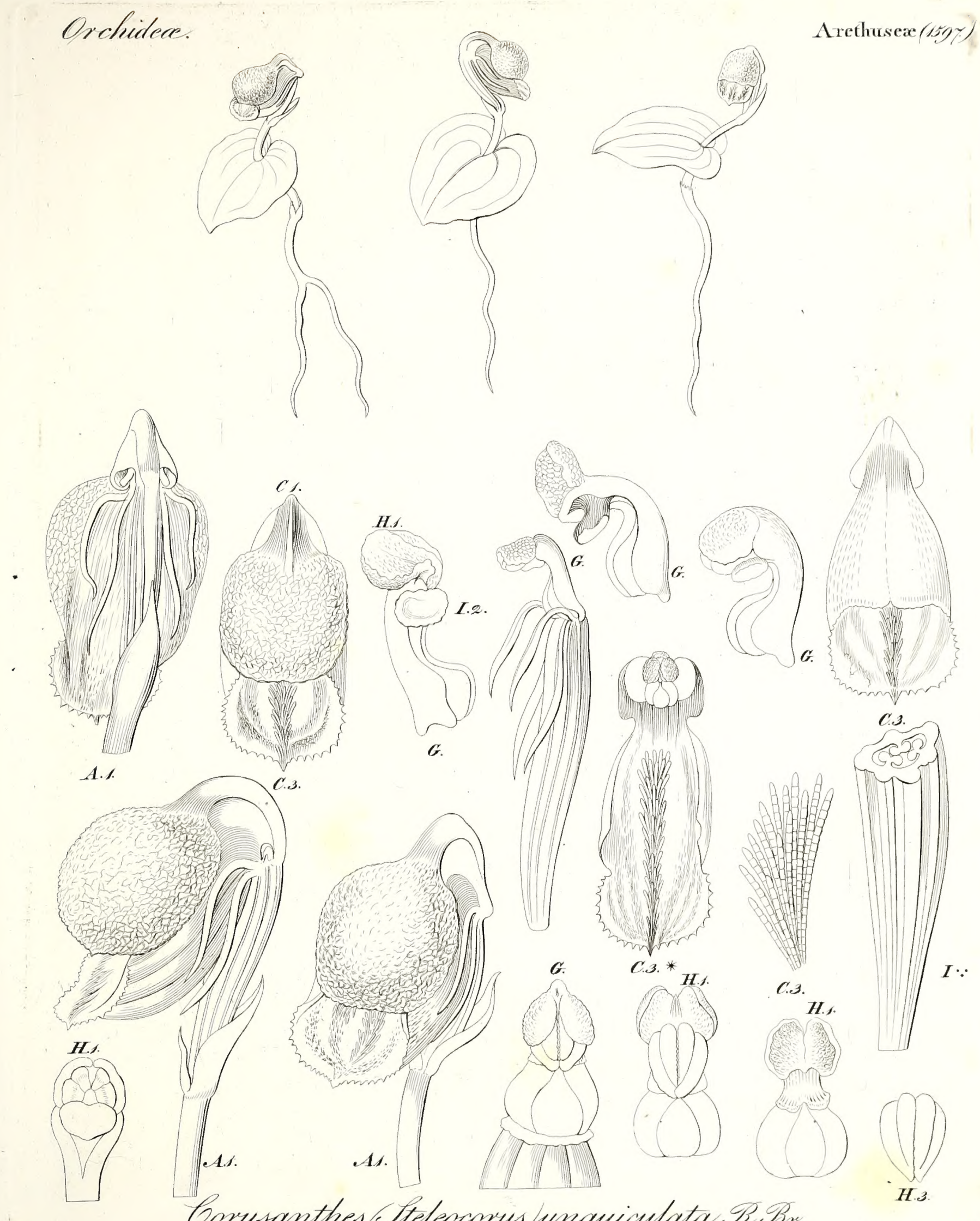

Gorysanthes Steleocorys unguiculata $\mathscr{R}_{R} \mathscr{B}_{2}$. 



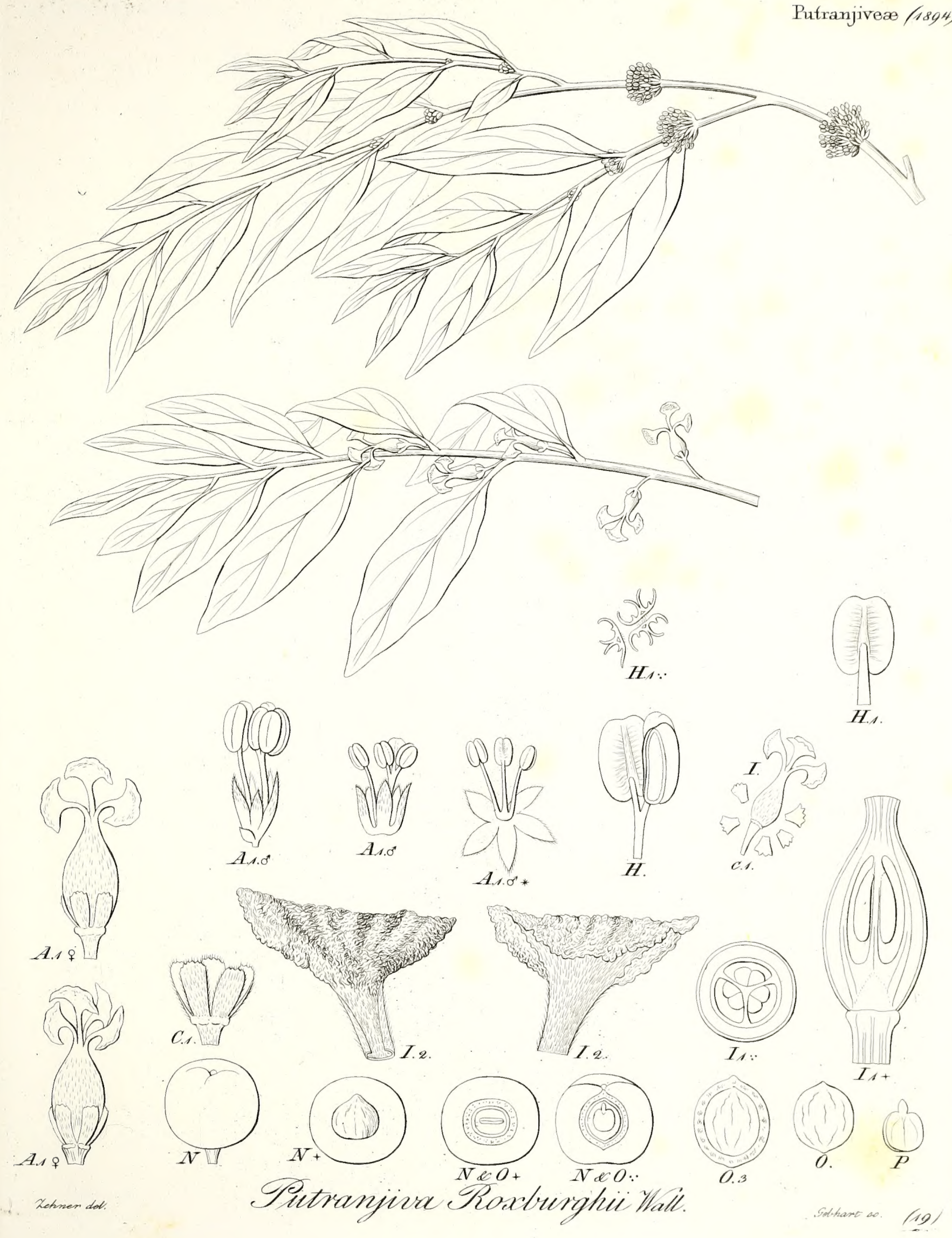


Amarantacece.

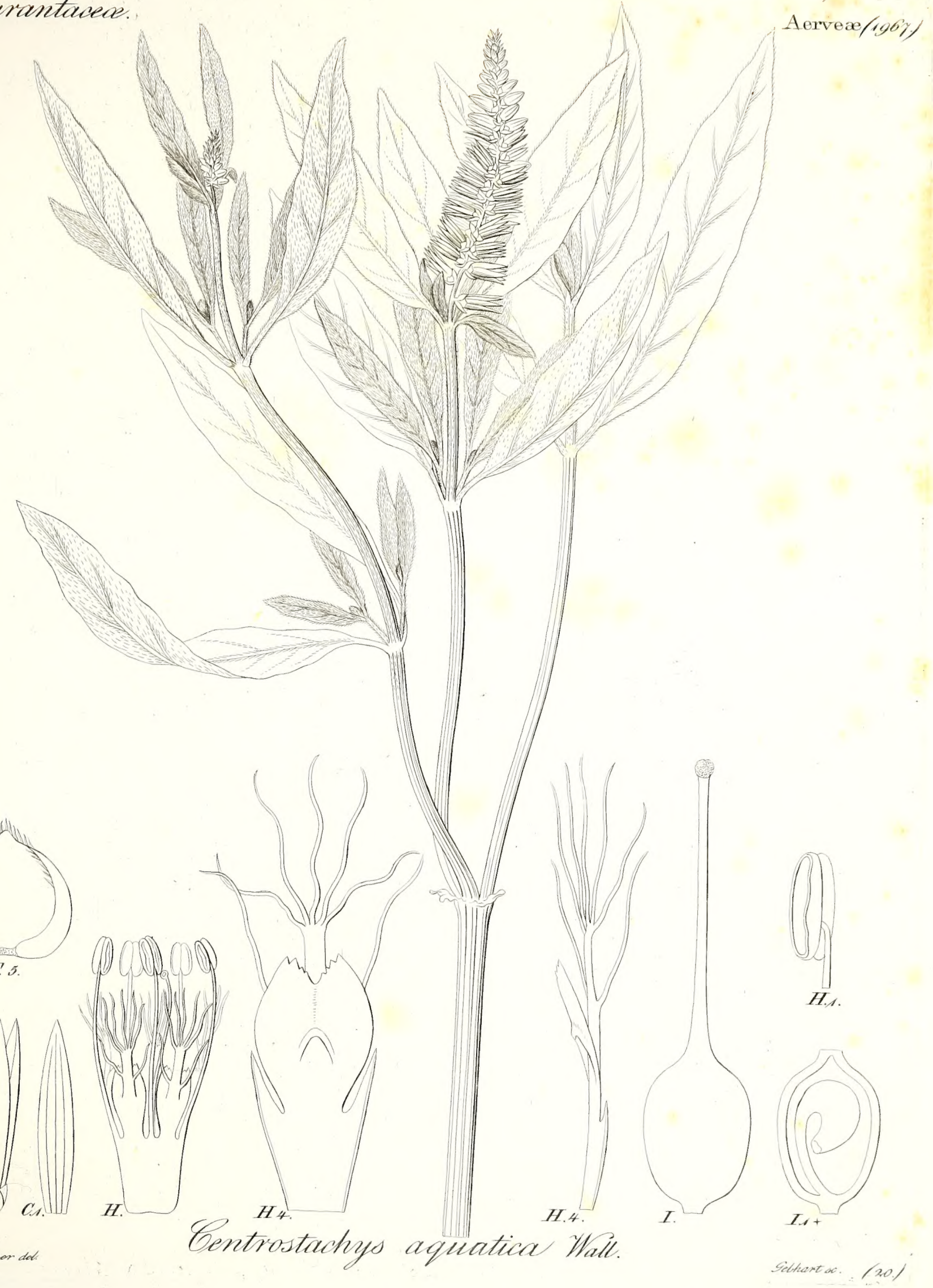



Monimiaccee.

Monimiex . (2019.

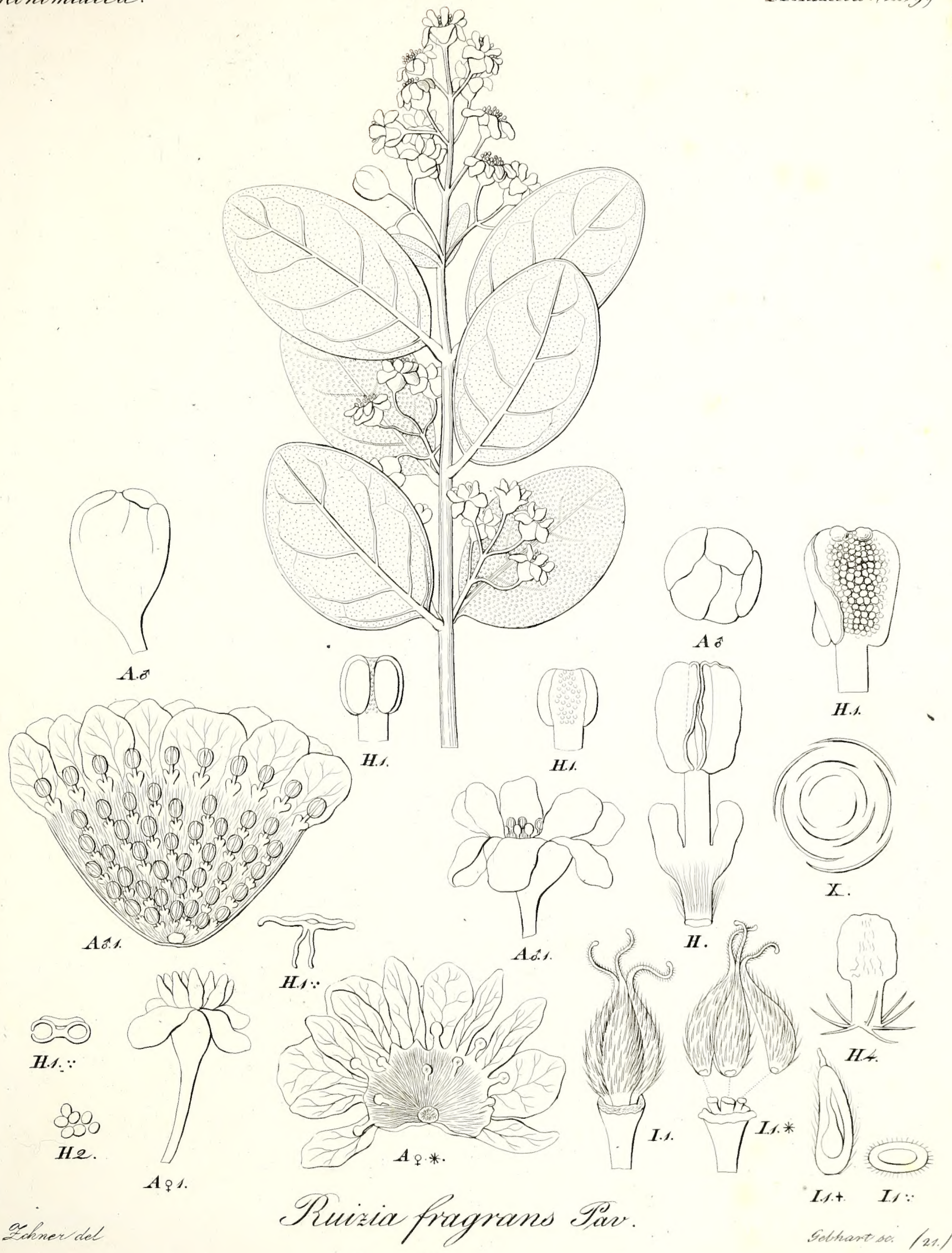



Saphnoidea.

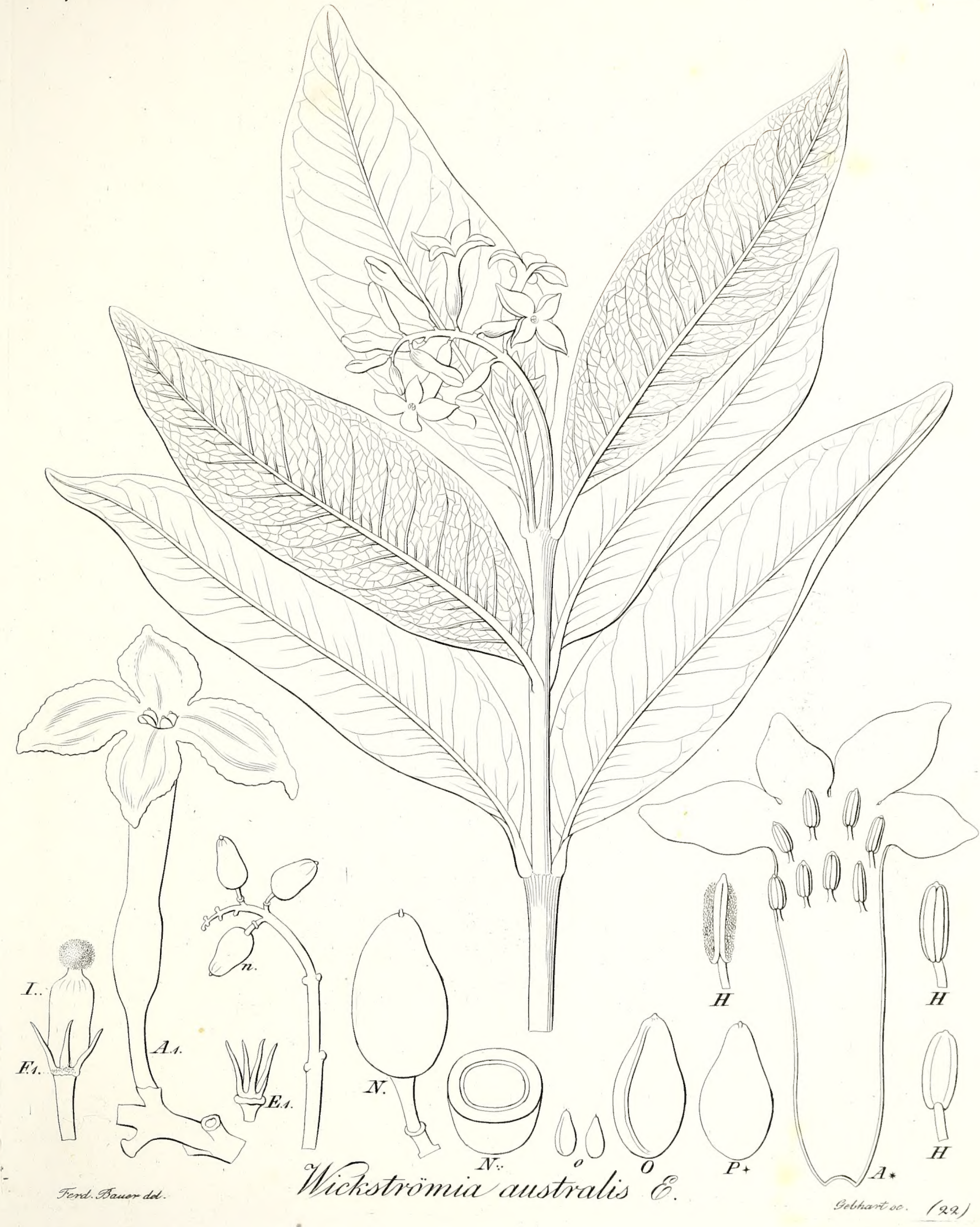


nge.$$
\text { . }
$$

4 


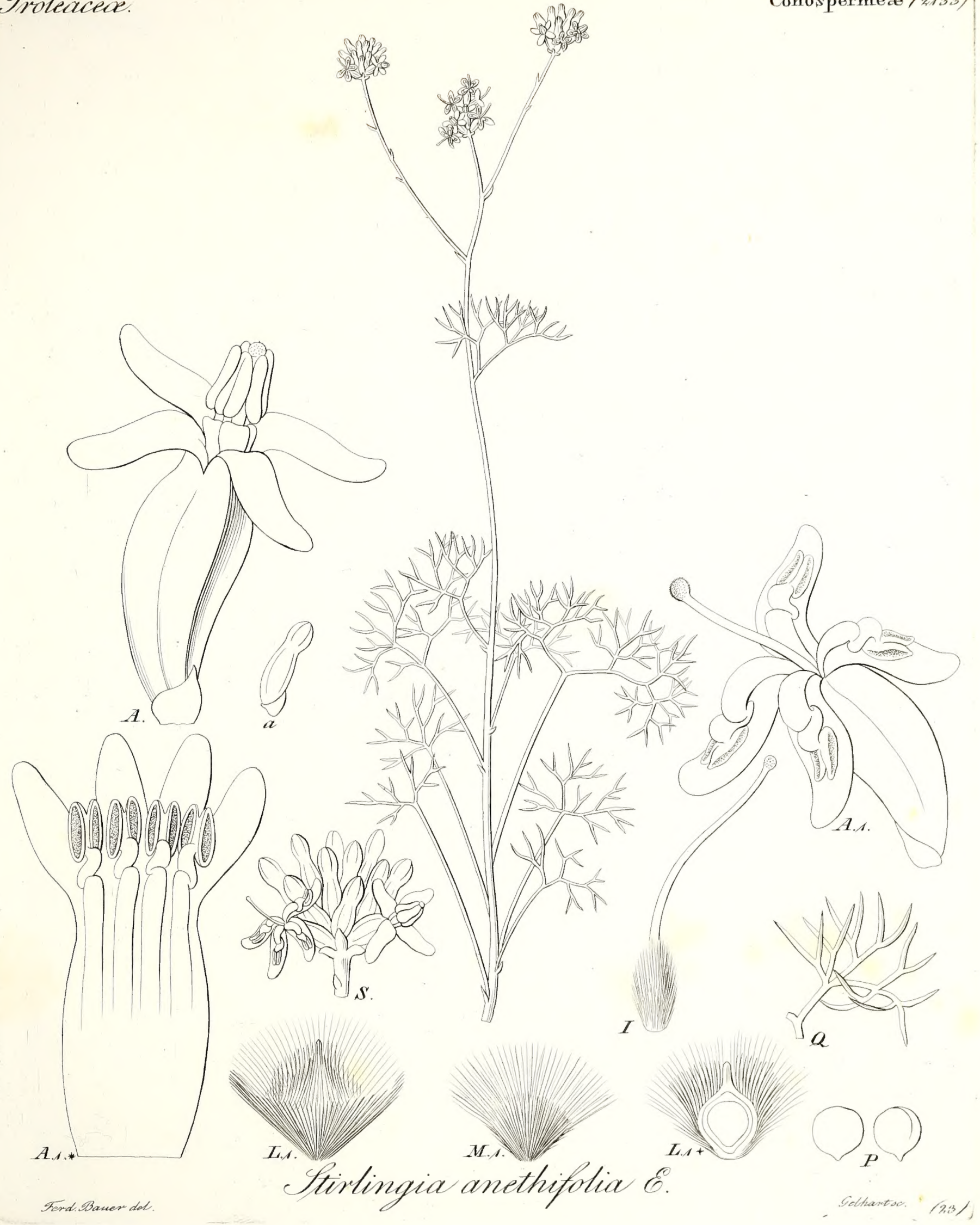





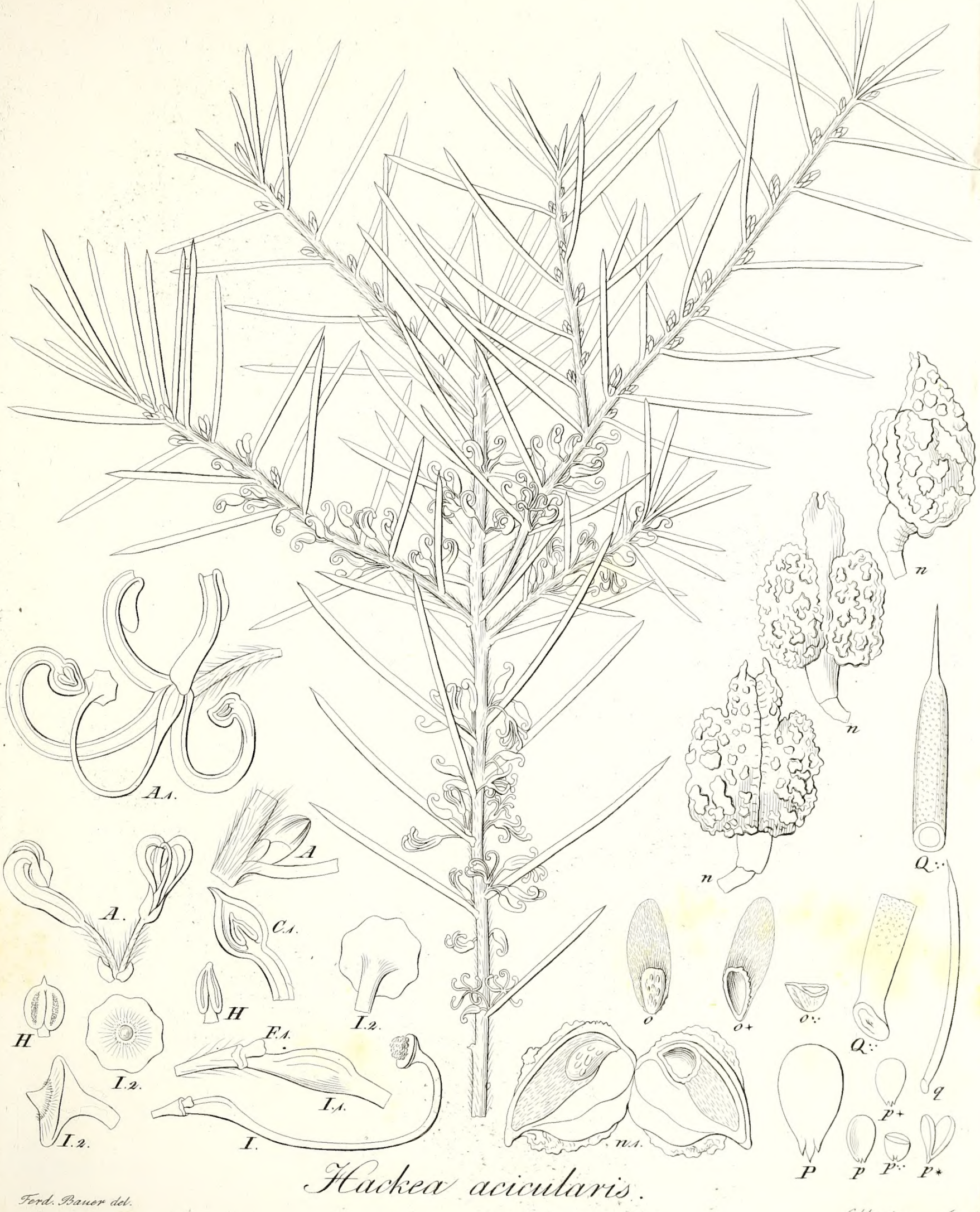




$$
\text { , }
$$



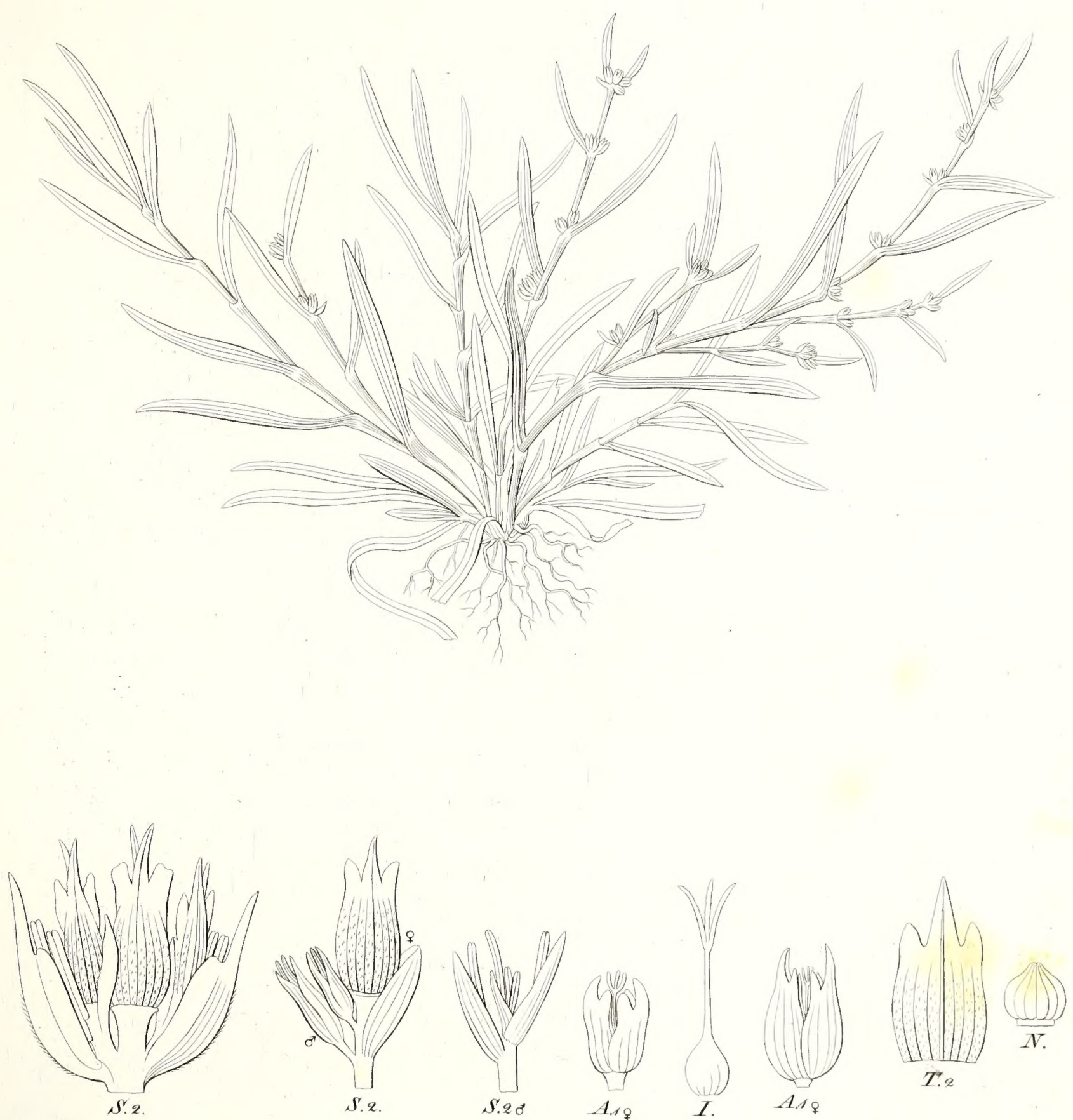

Ferdiscouer dol.

Diplacrum caricinum R.B. 


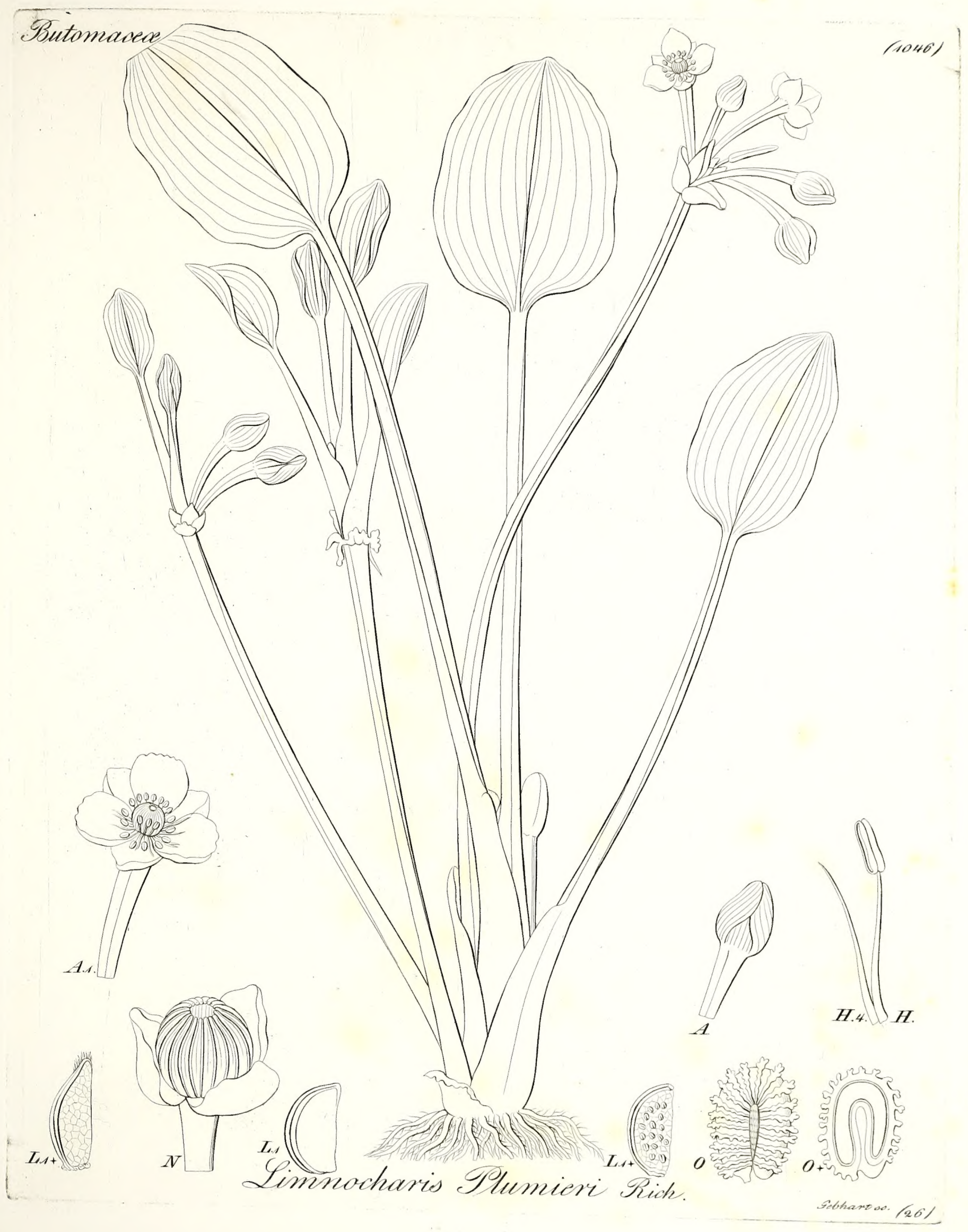




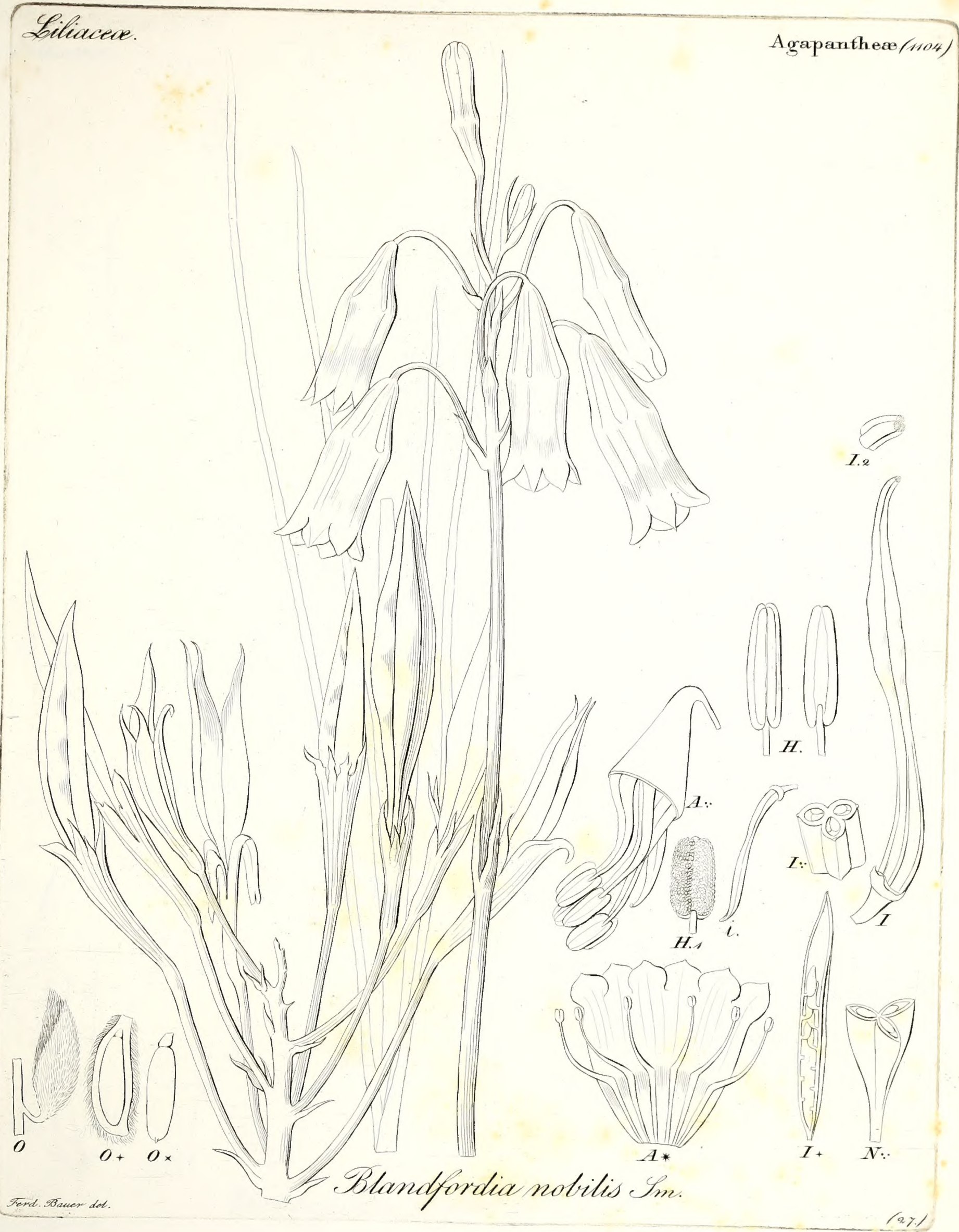




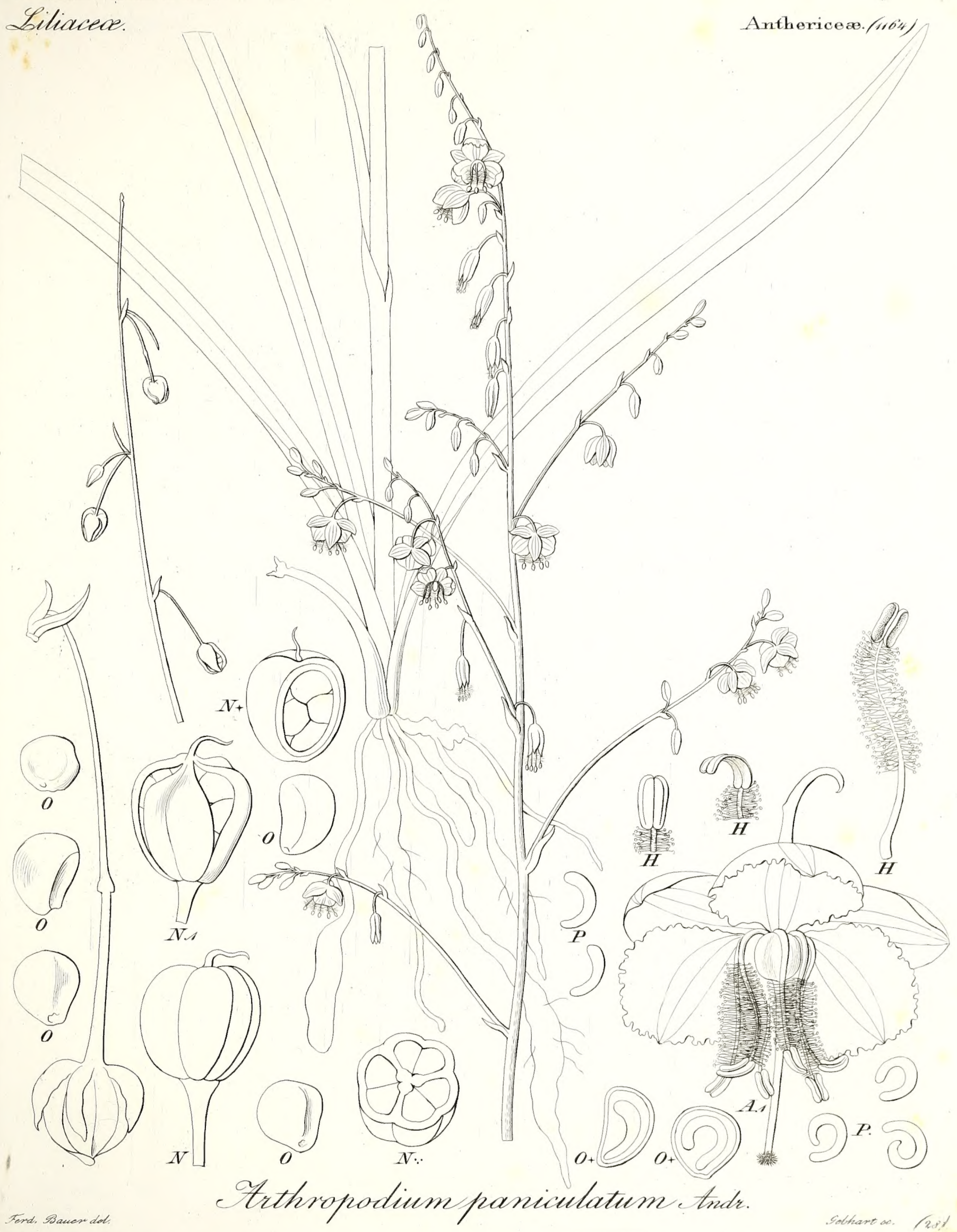


$\therefore \quad \therefore$

$\because$

:

$\therefore \quad$

$\vdots$

$\because$

$\therefore$

$\because$

$\therefore$

$\therefore \quad$ :

$\because$

$\because \quad \therefore$

$\therefore$ 
Orchidece.

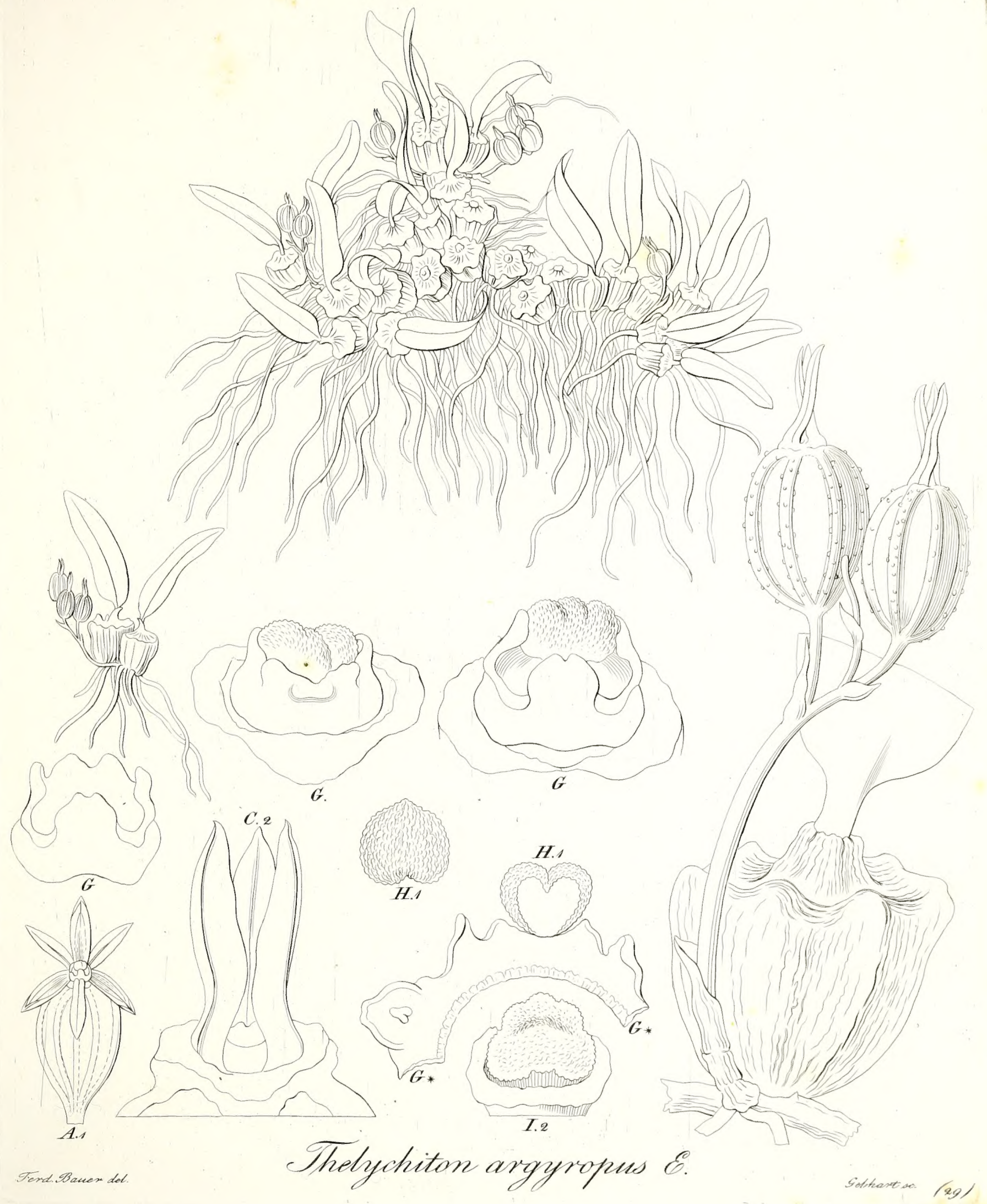





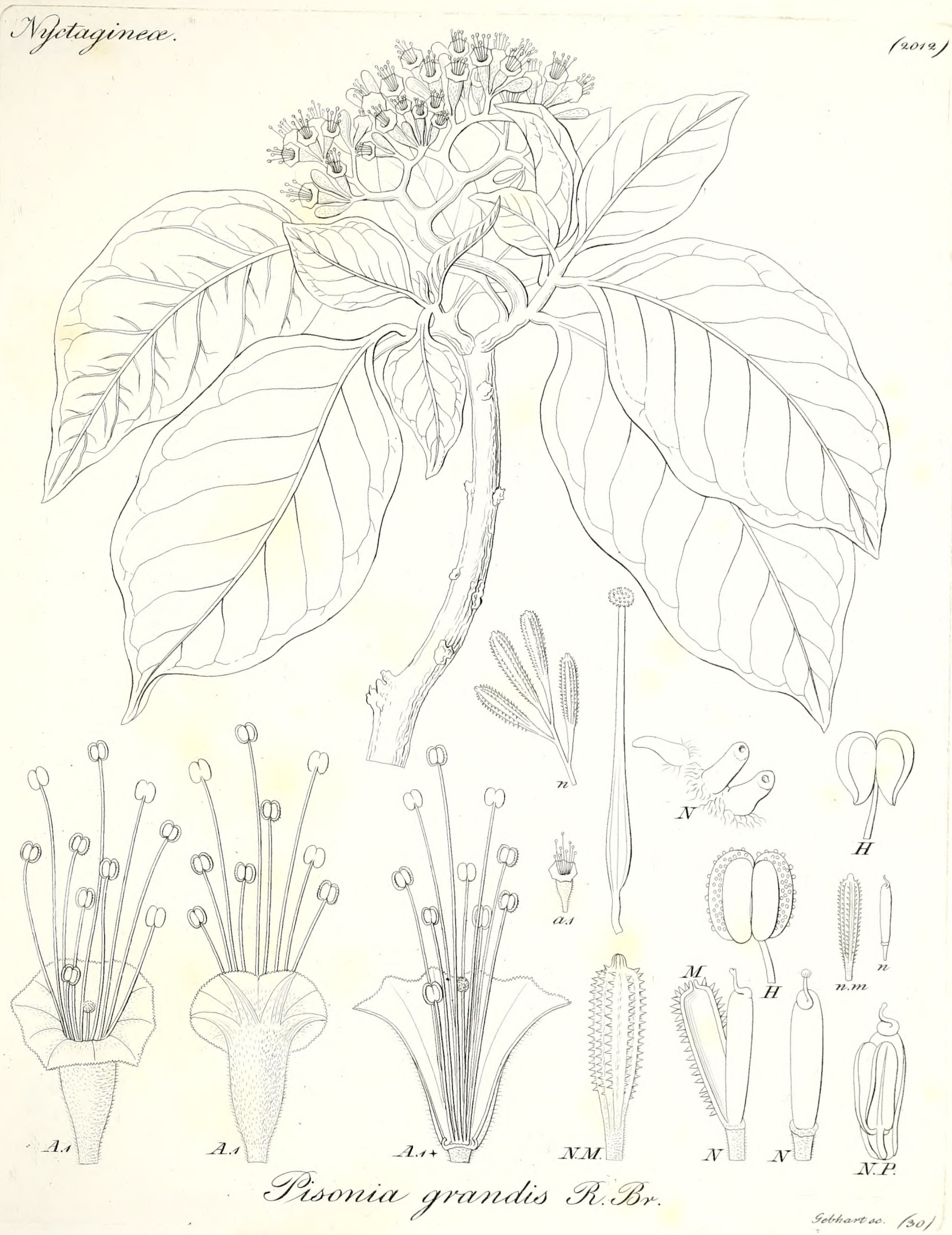



Proteacea.

Conospermea/2132)

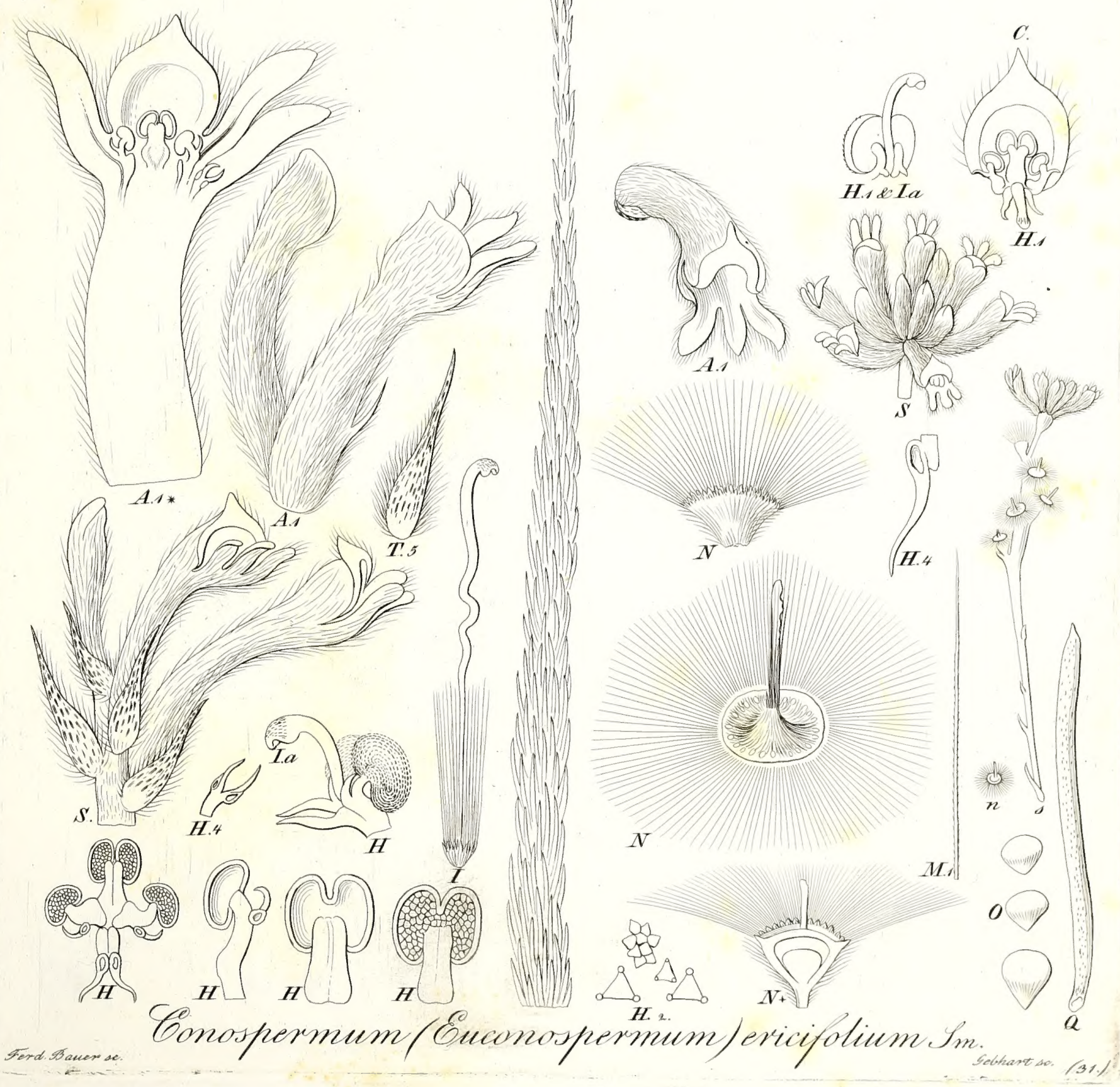


Proteacea.
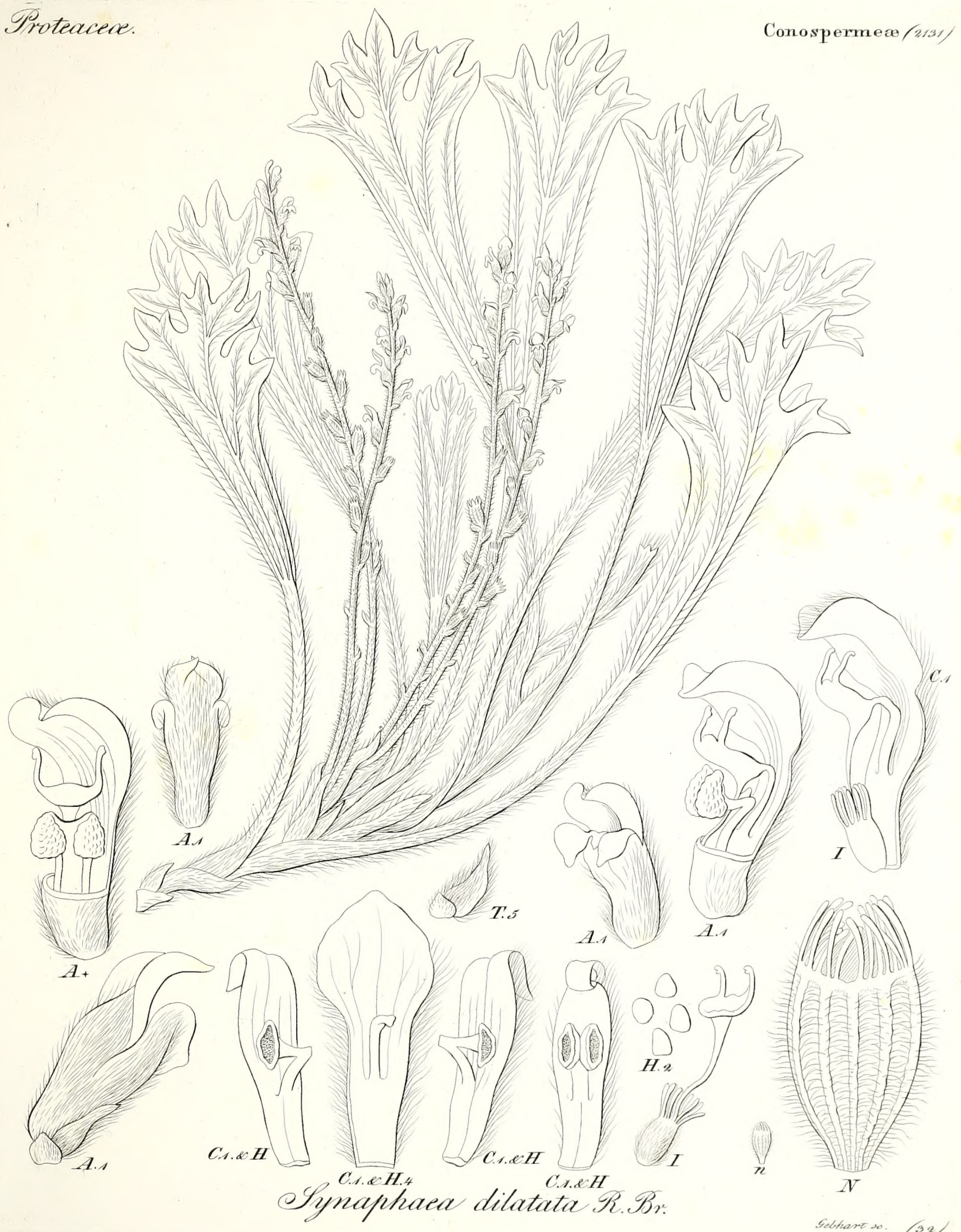



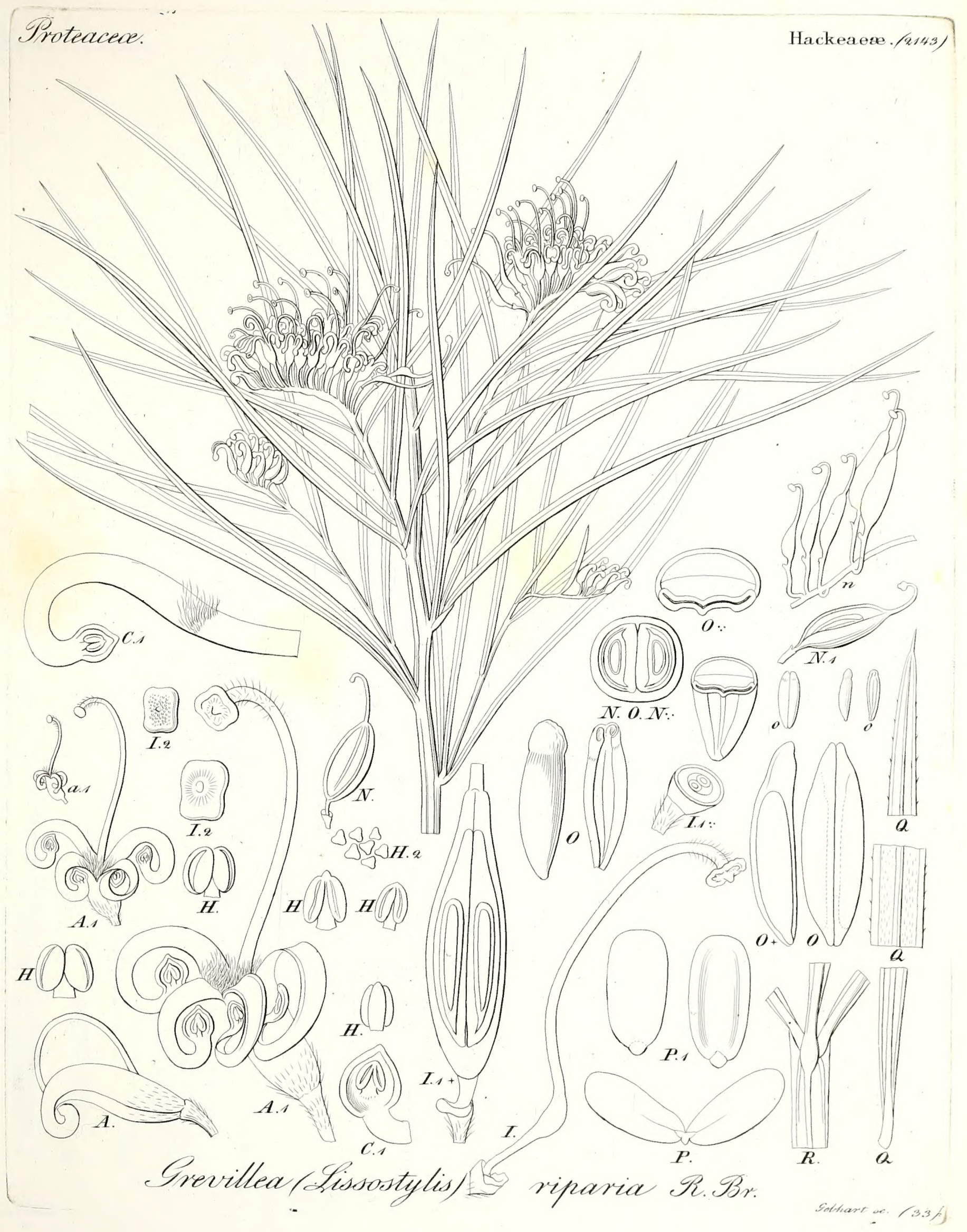


$\therefore \quad$

Vernoniacex.

Vernonieas/2213)

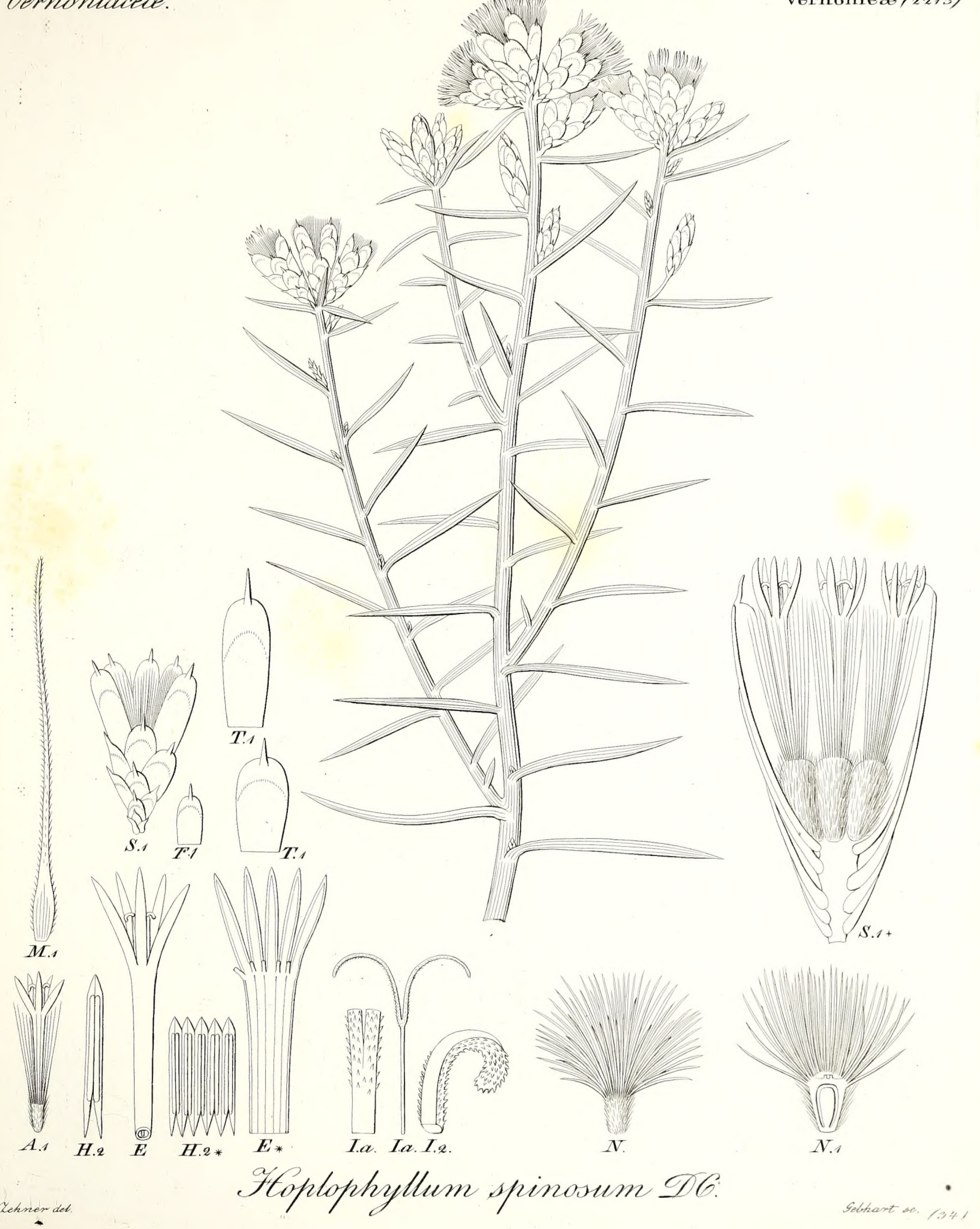




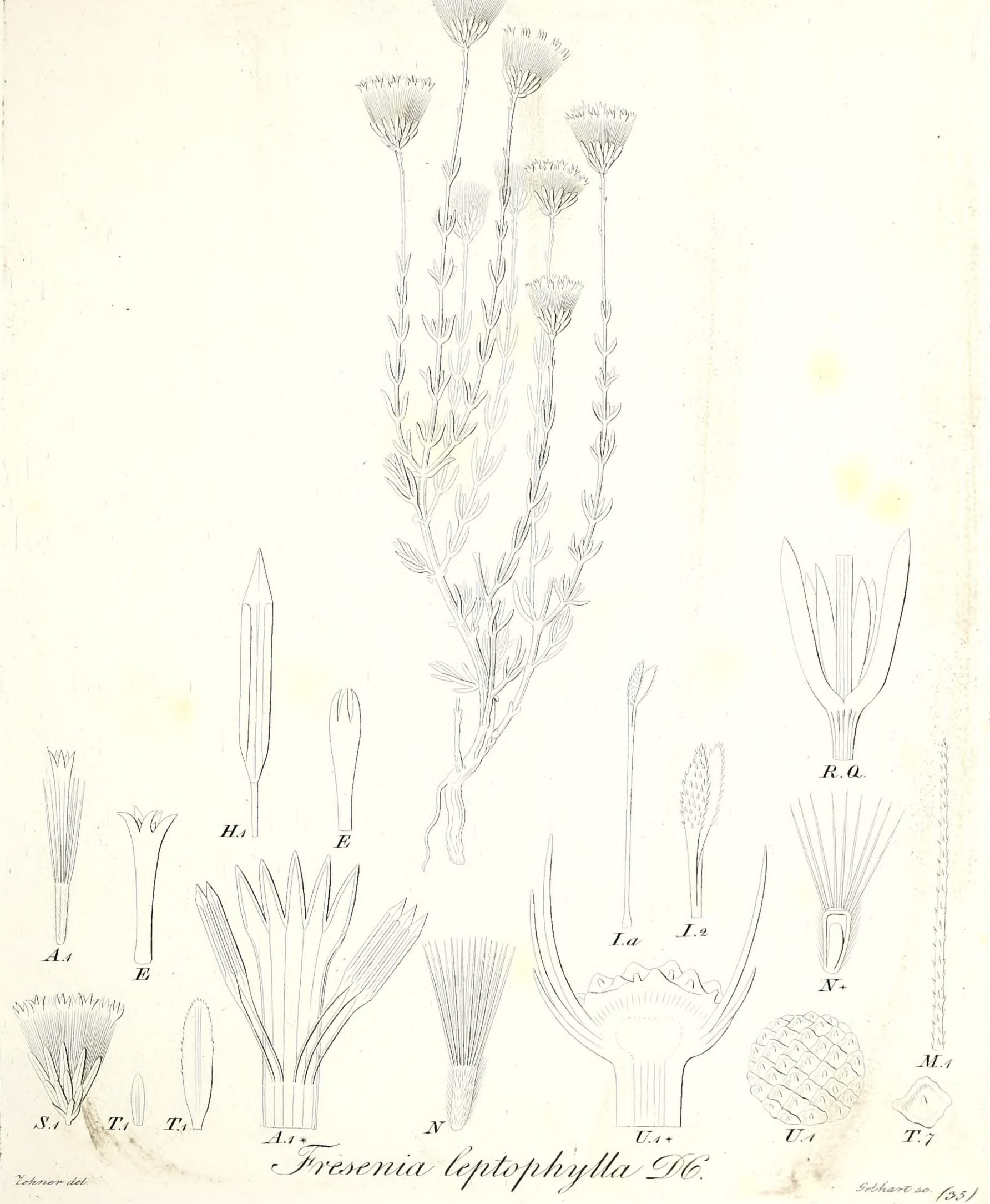



Senecionidea.

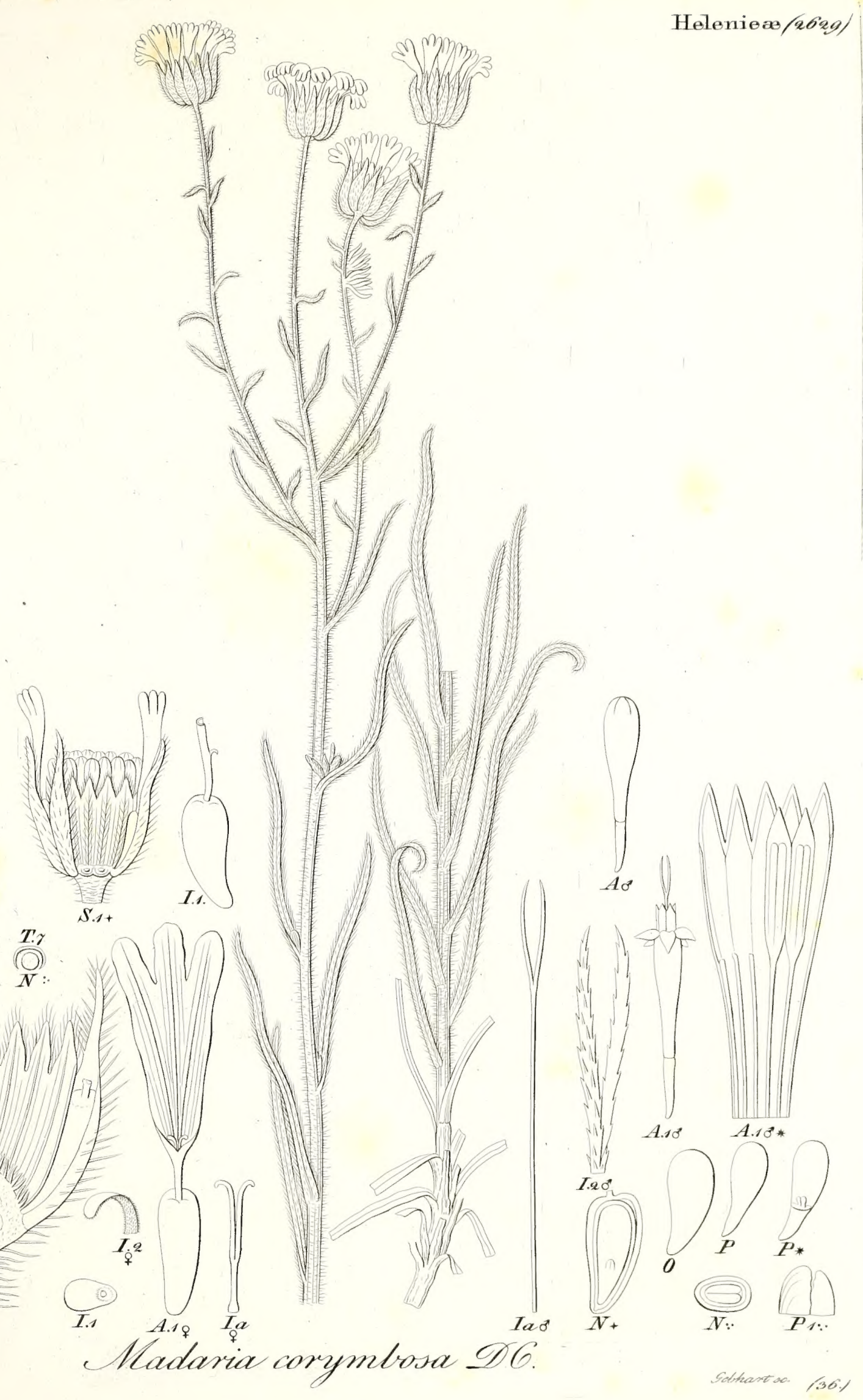




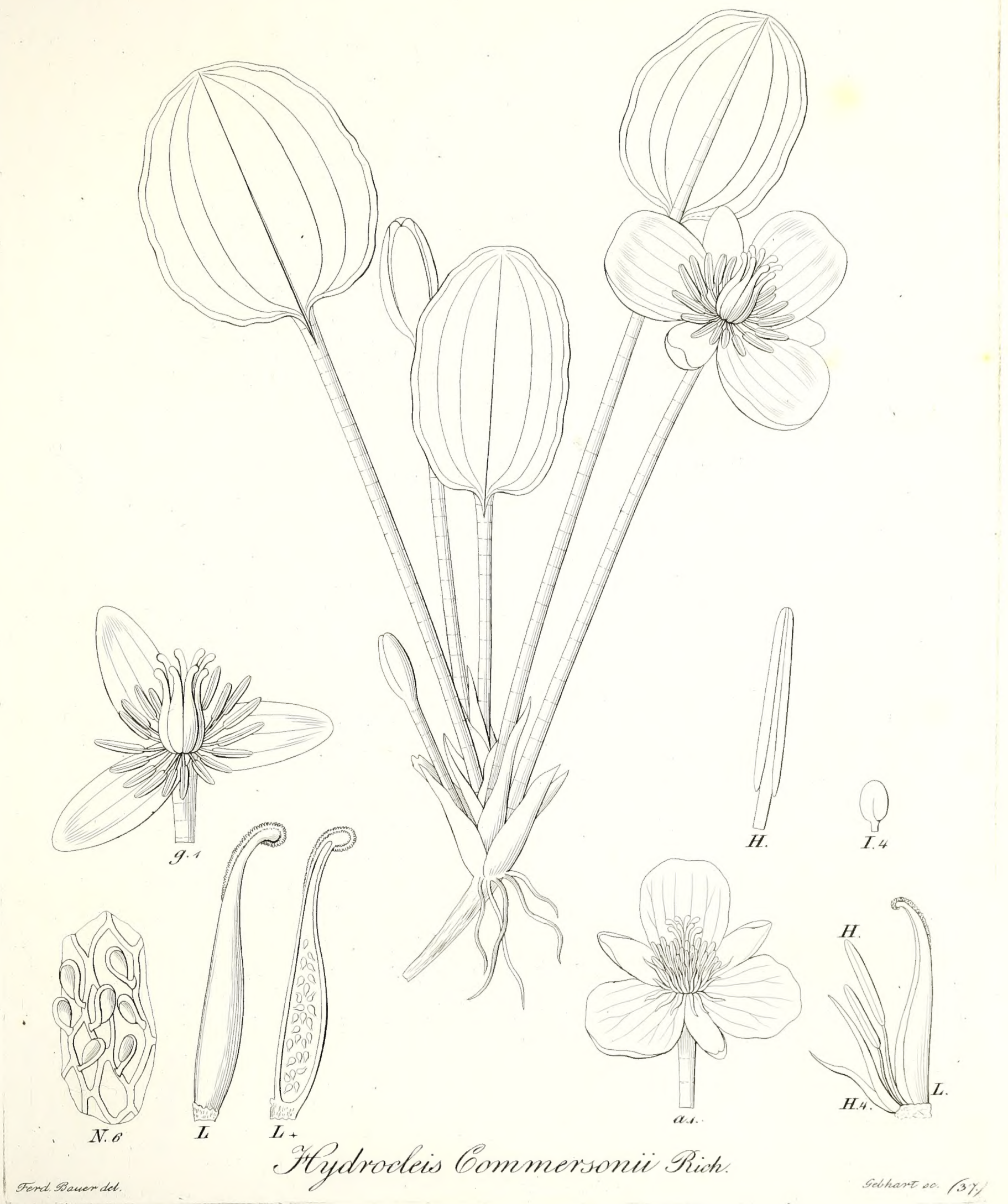




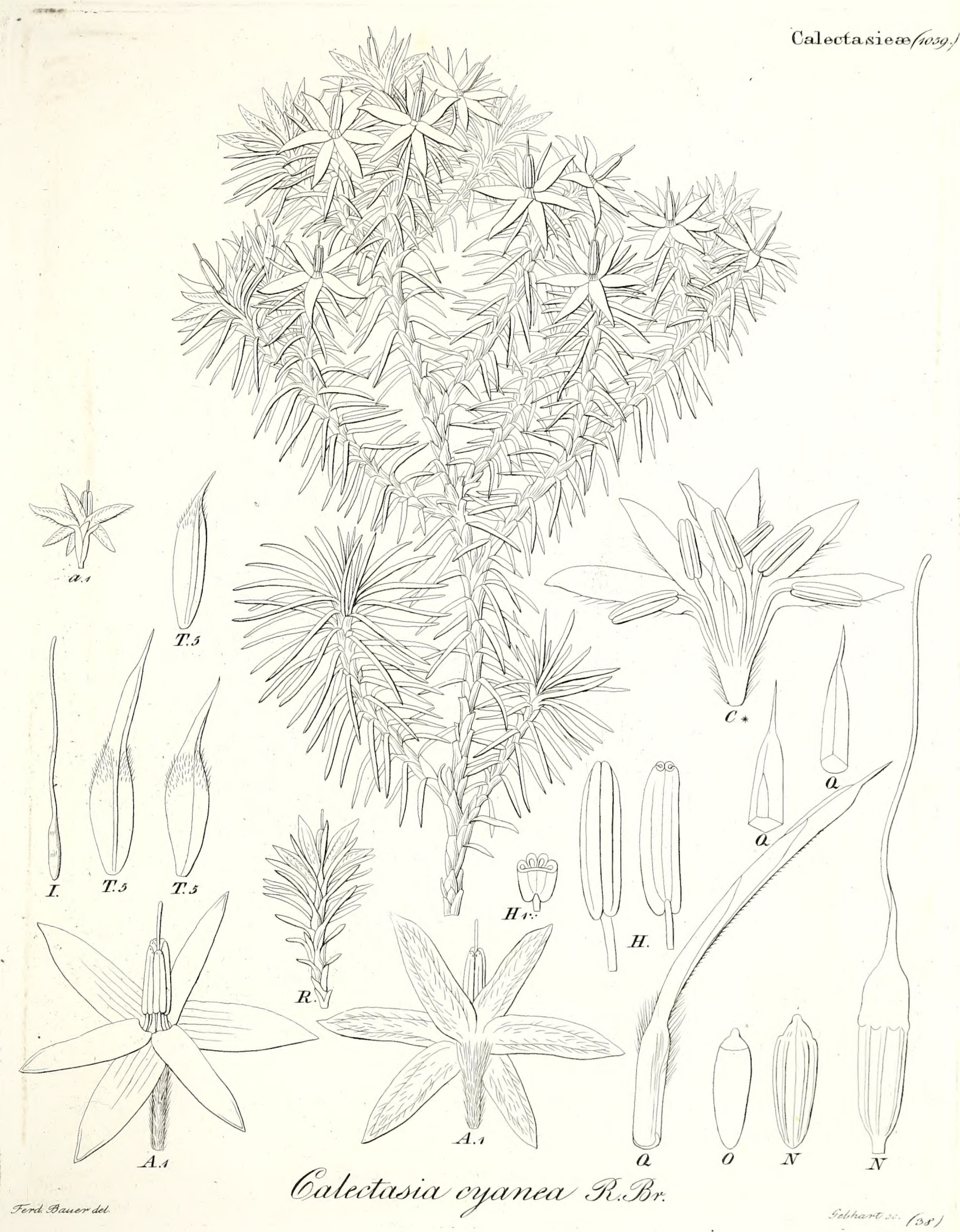



Smilacece.

Convallarieae (ust)

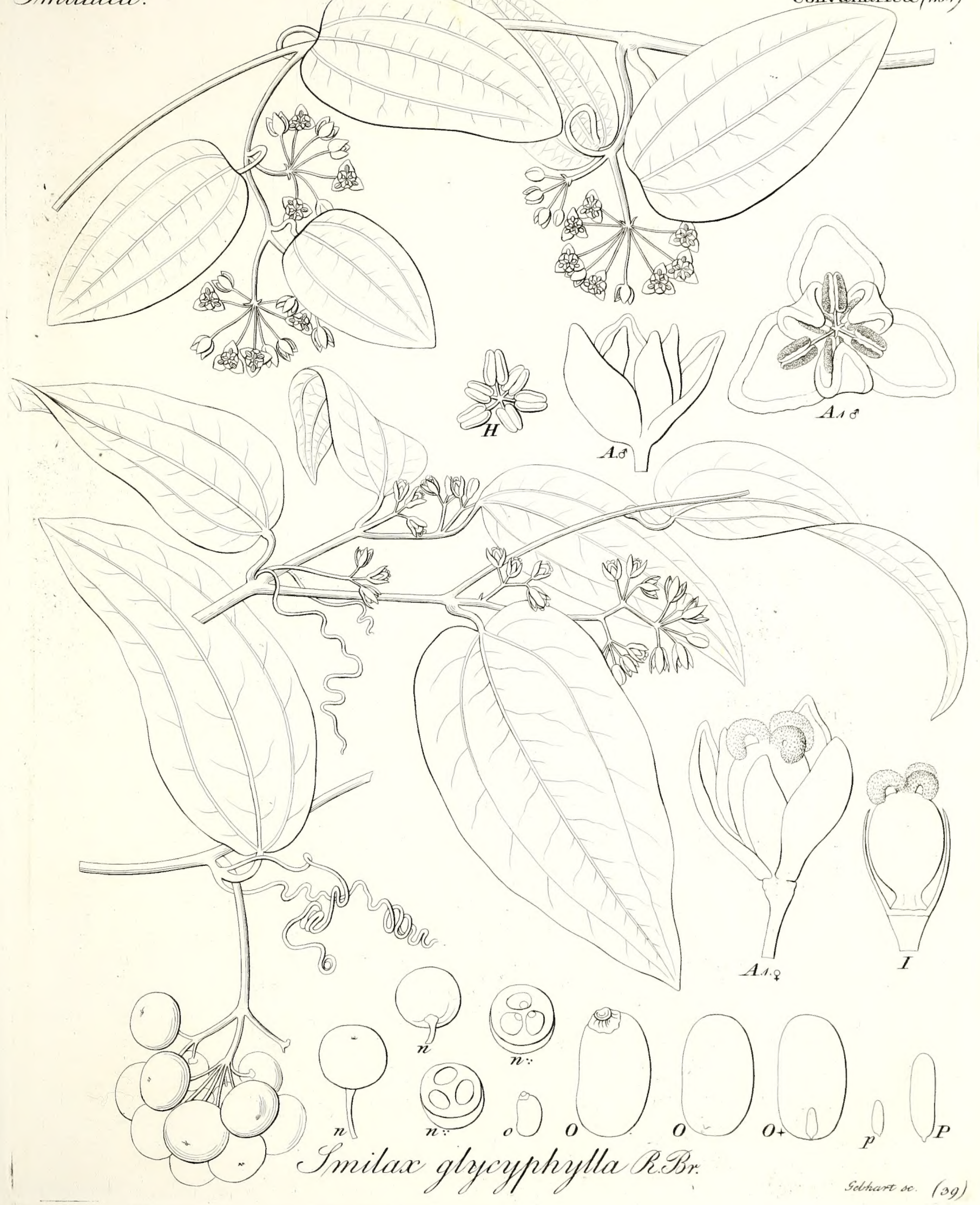



Orchidece.
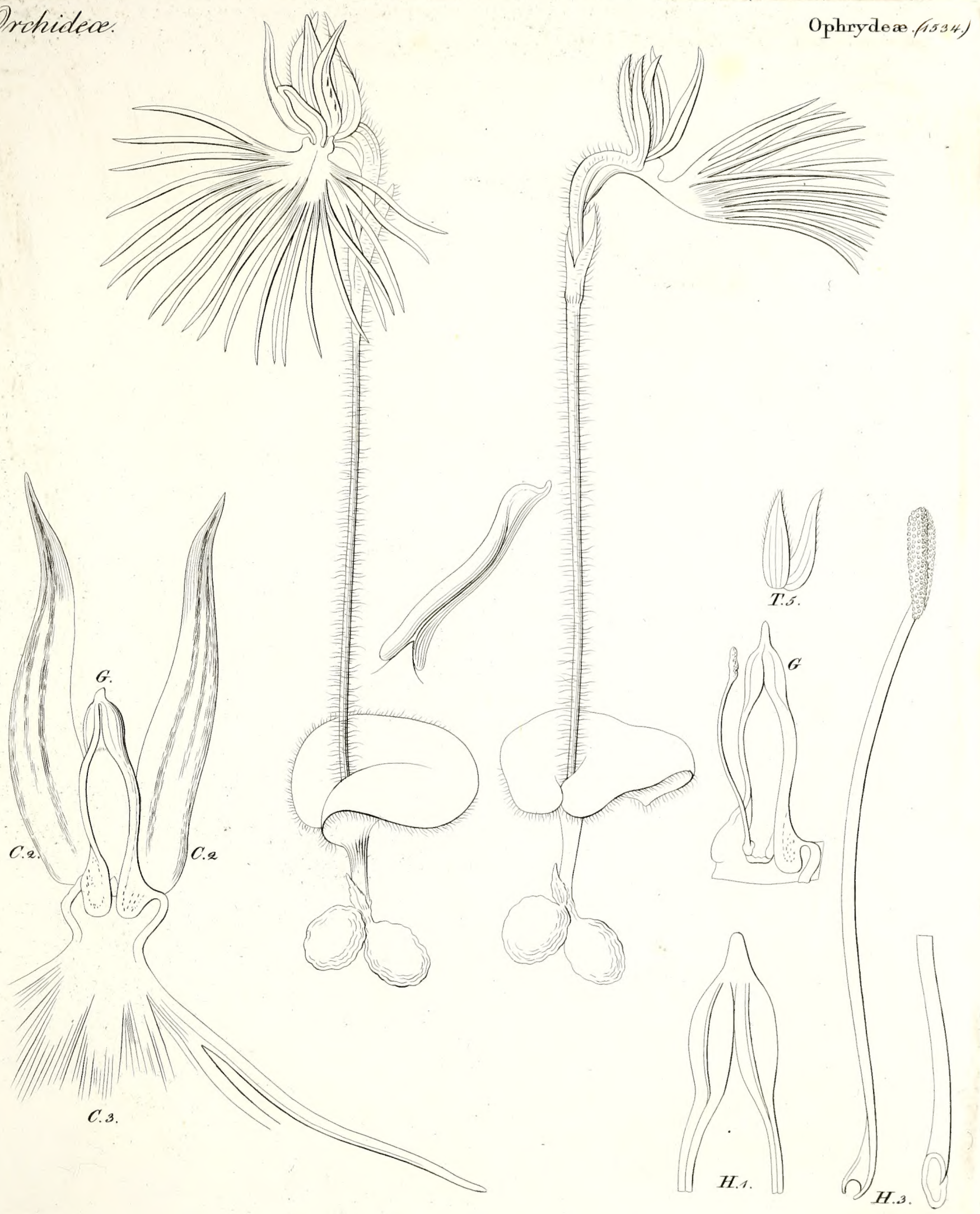

Bartholina pectinata Sindl. 
Orchidece.

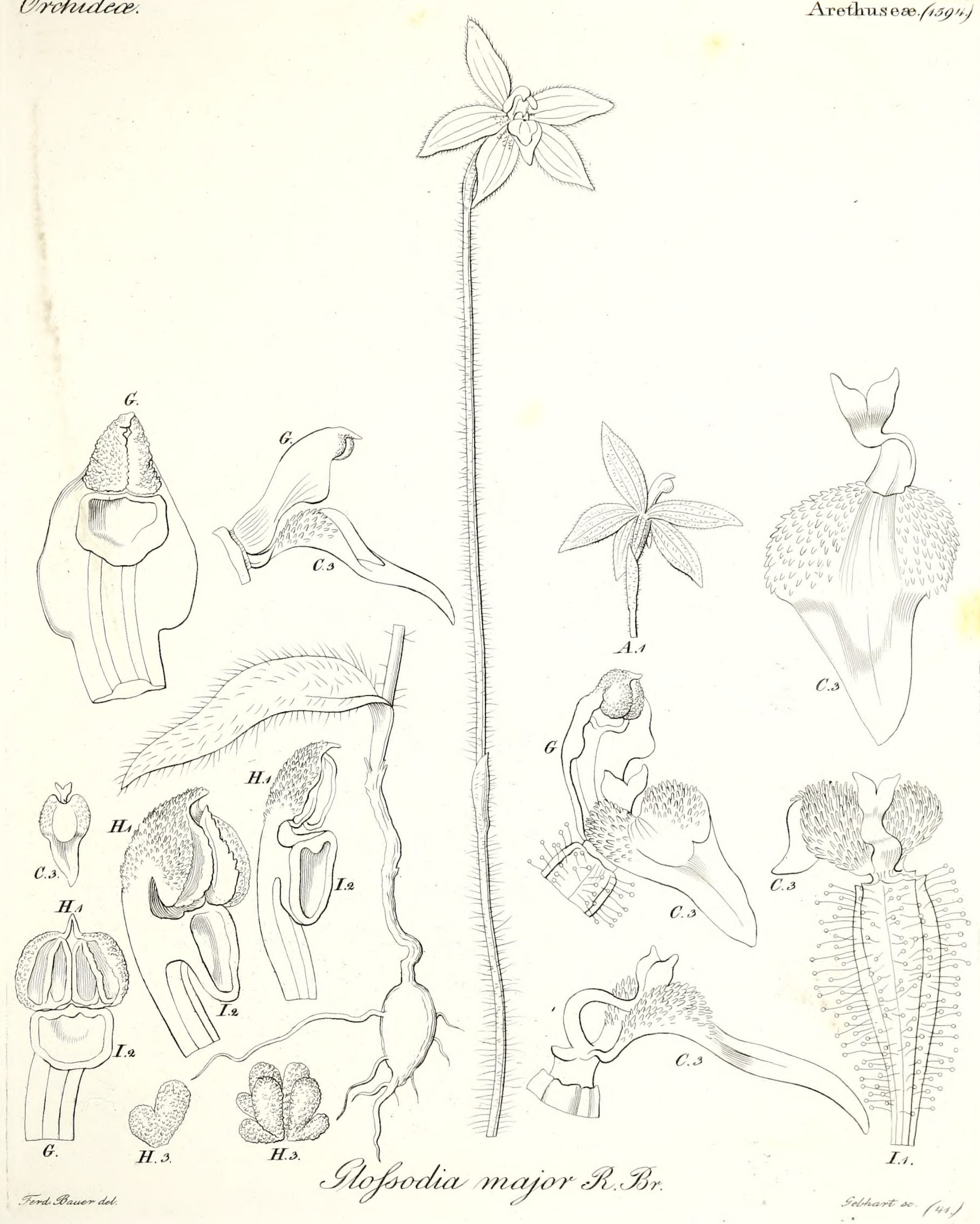


Nusacede.
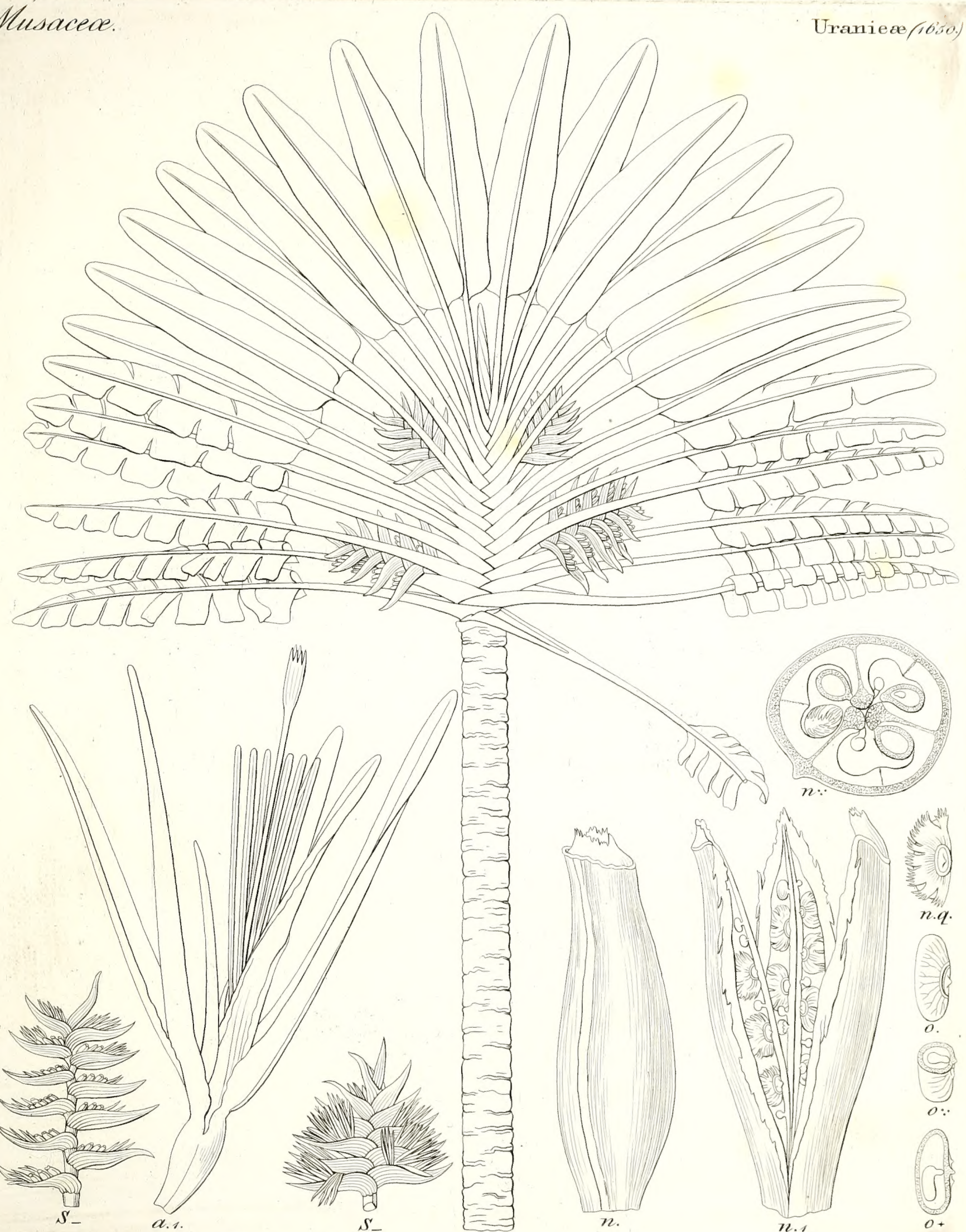

Ravenala madagascarieusis Sonner. 

Gyrocarpea.

reors

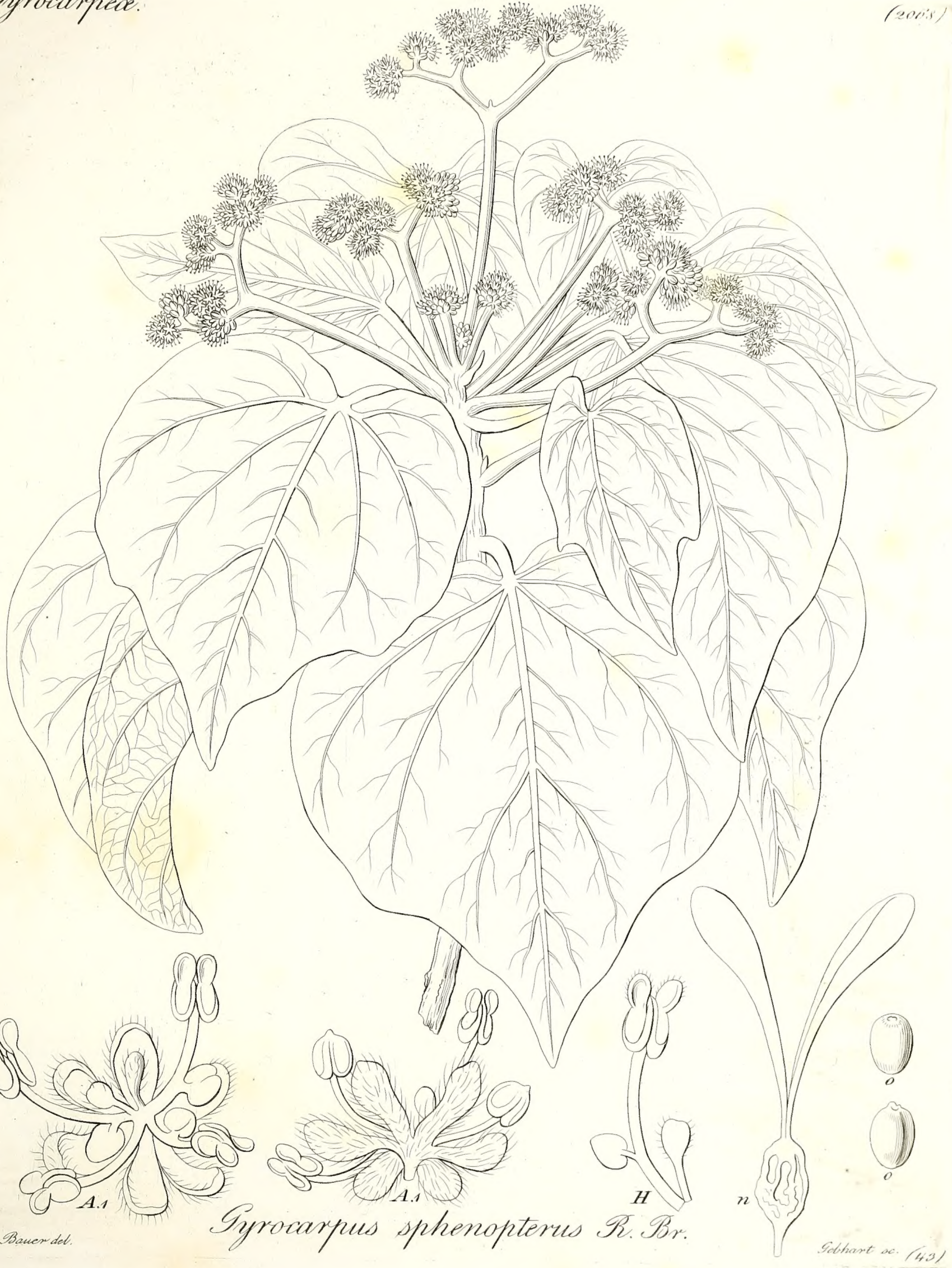





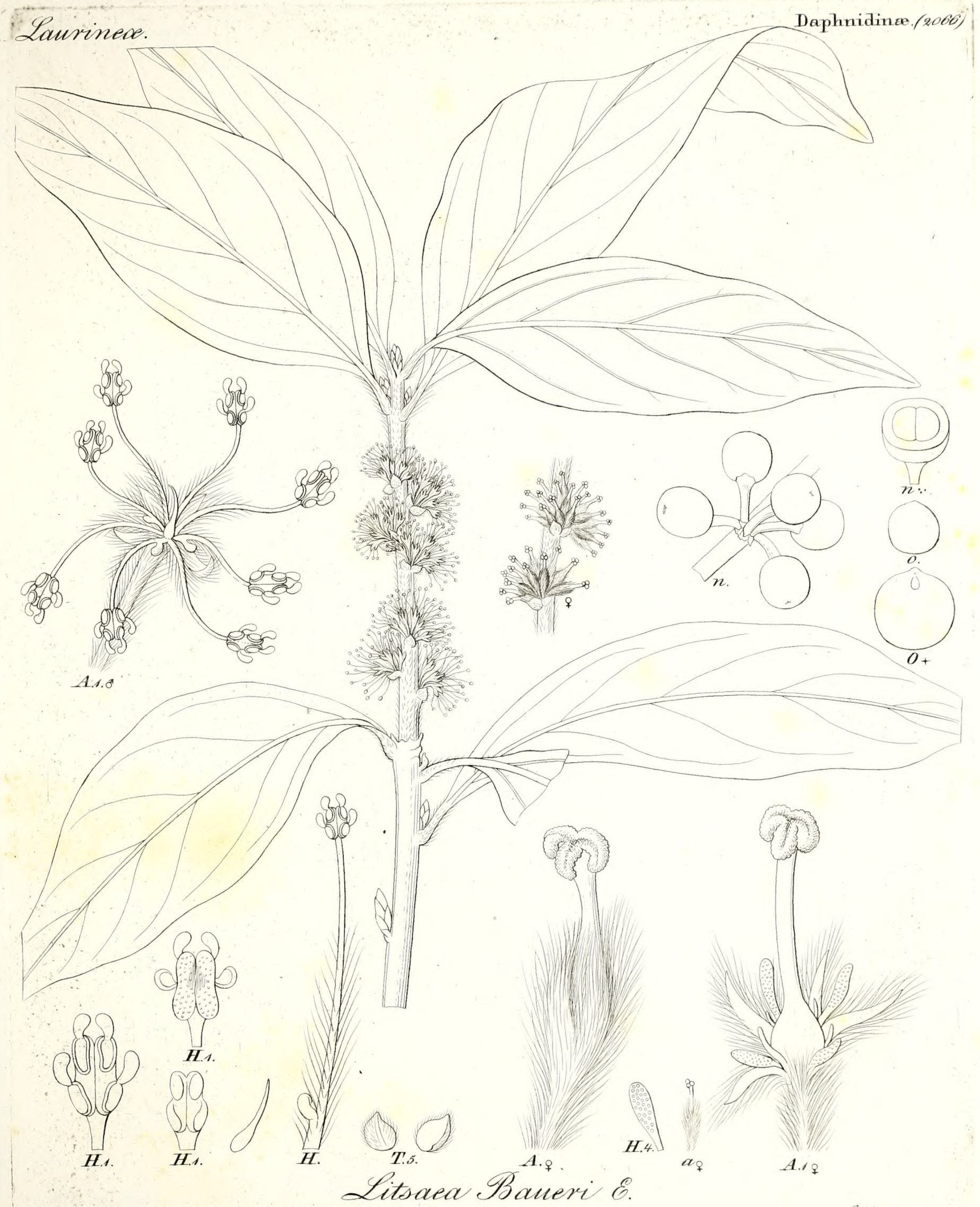


Santalacede. 


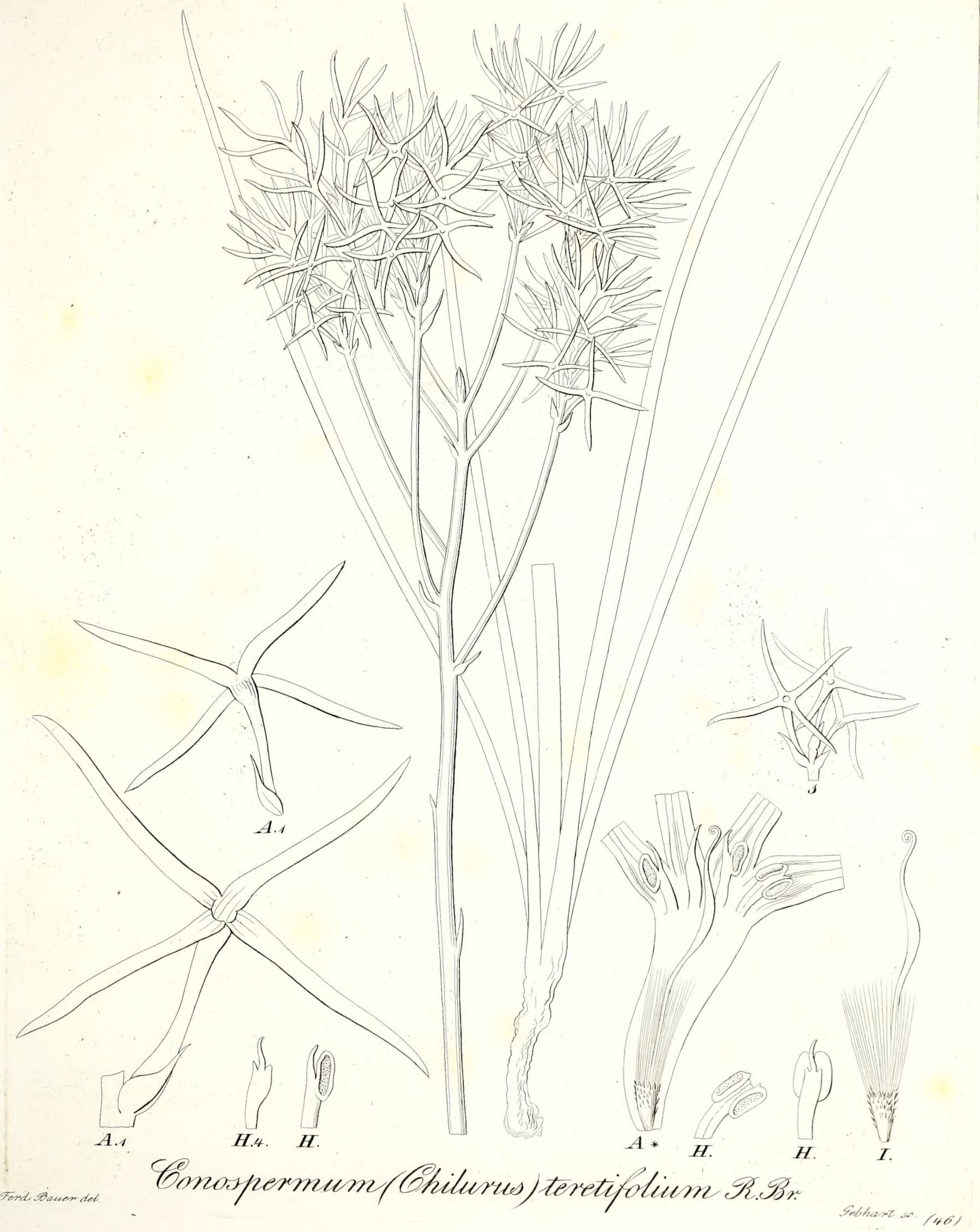





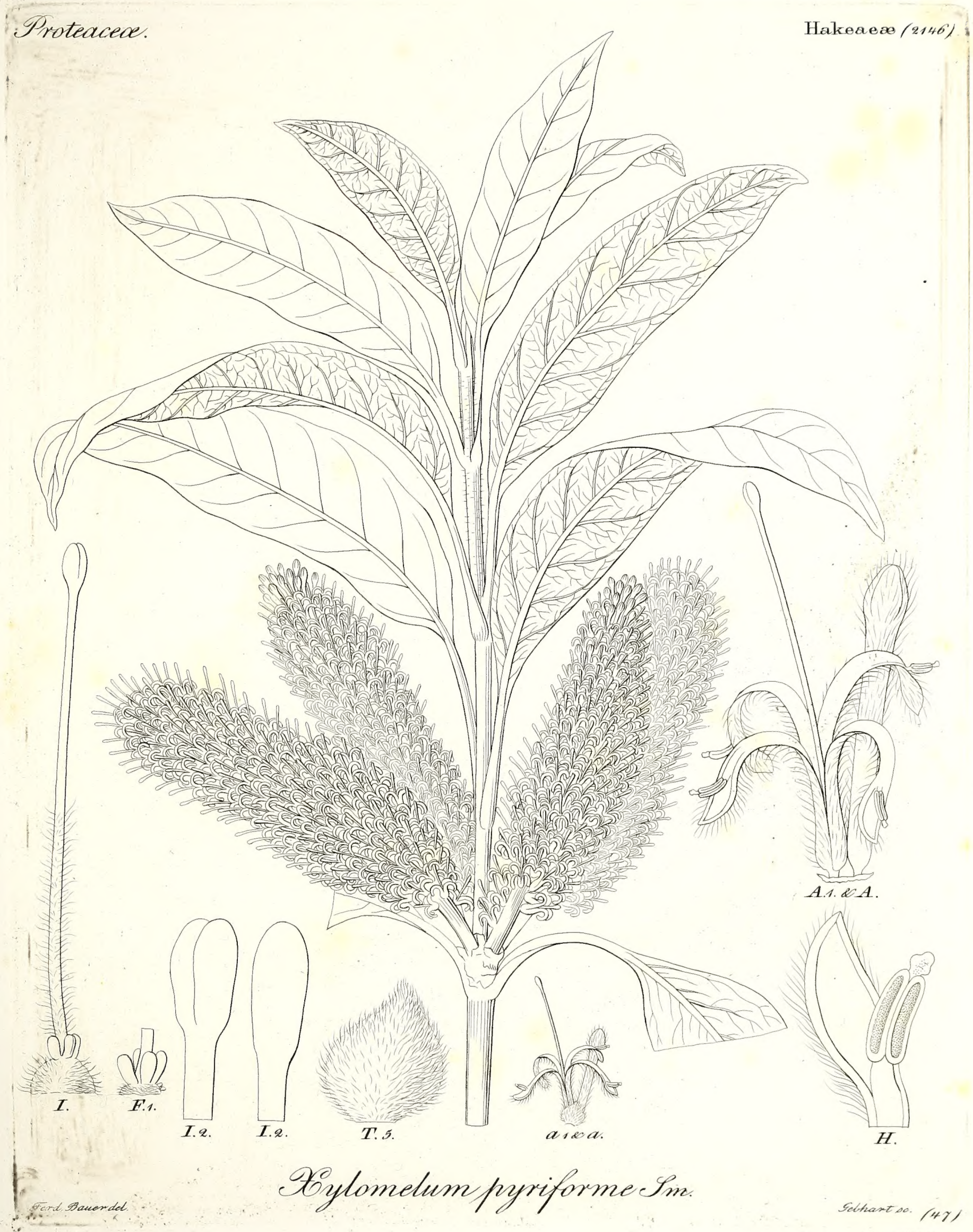





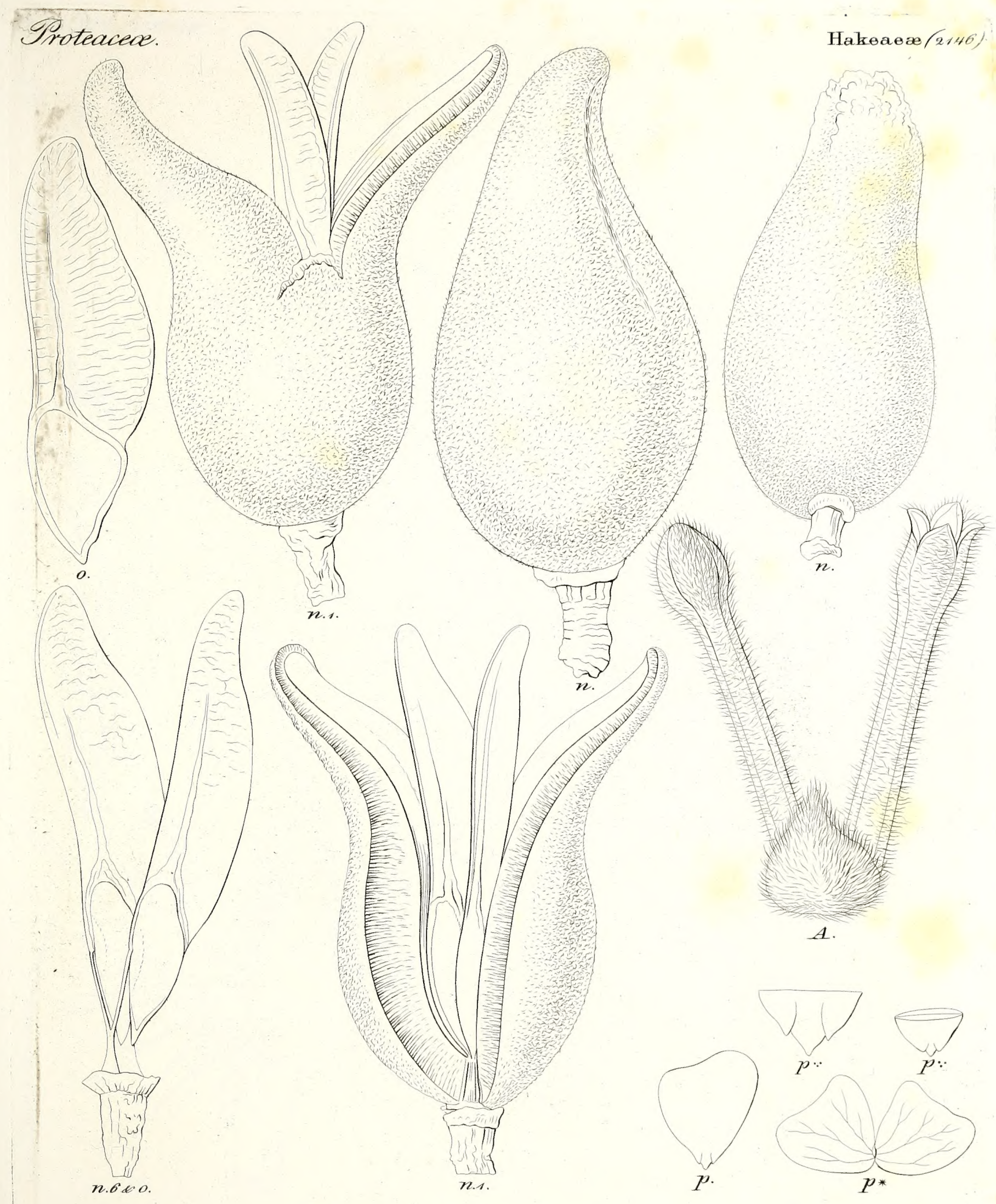

Doylomelum pyriforme $9 \mathrm{~m}$.

Sebrant ac. $(48)$ 

Gentrolepidece.

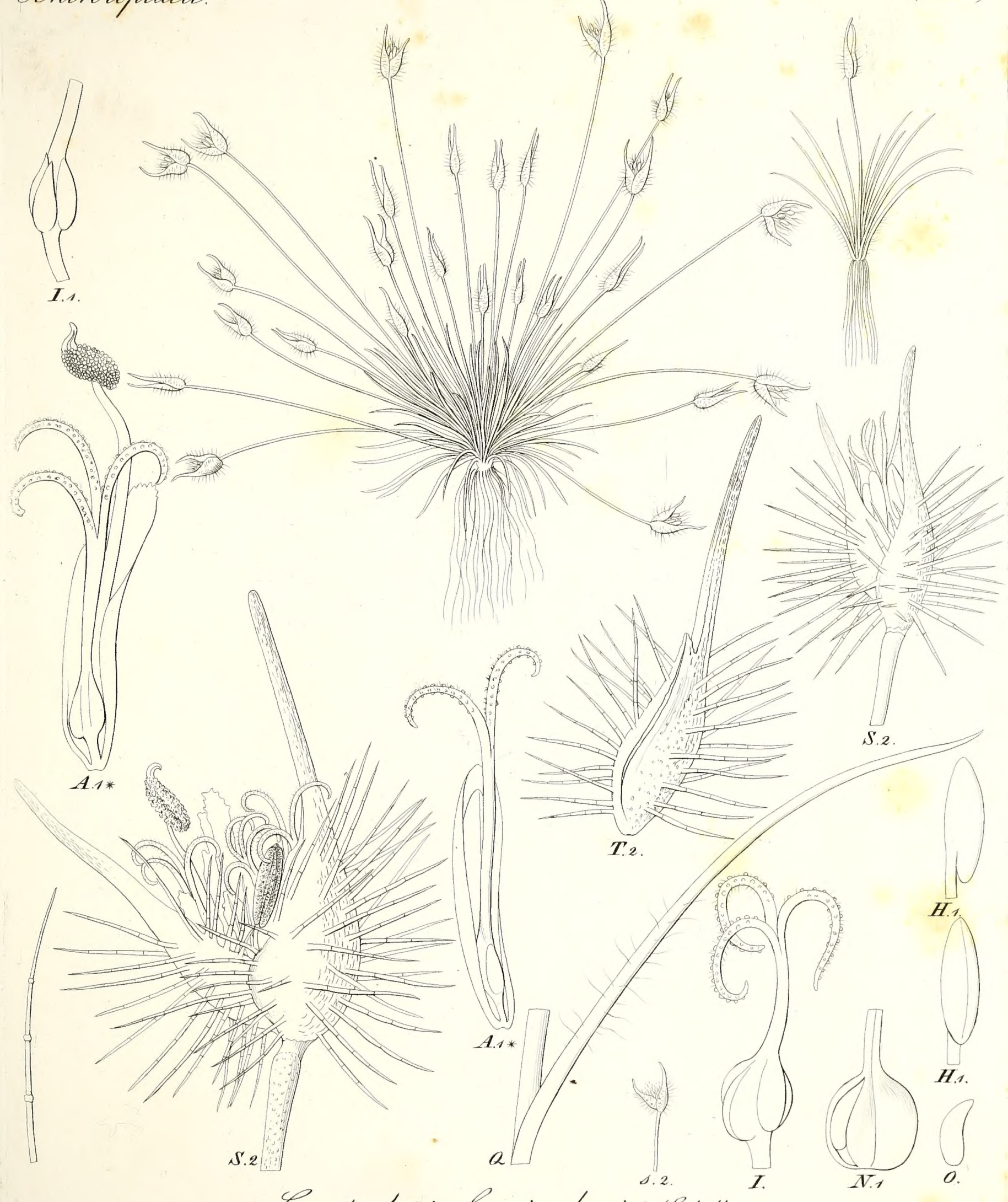

Gentrolepis fascicularis Labill. 


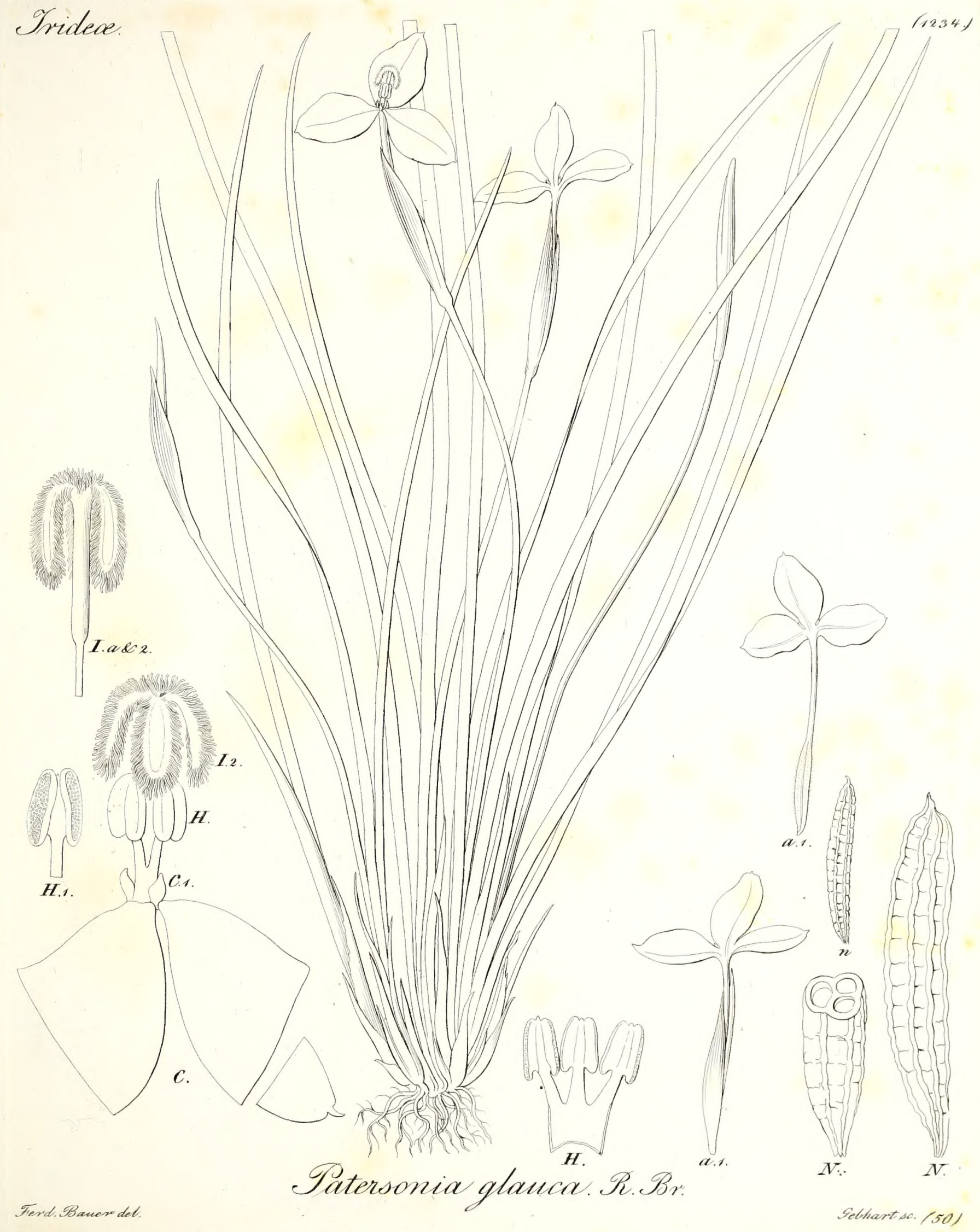


Orchidew.

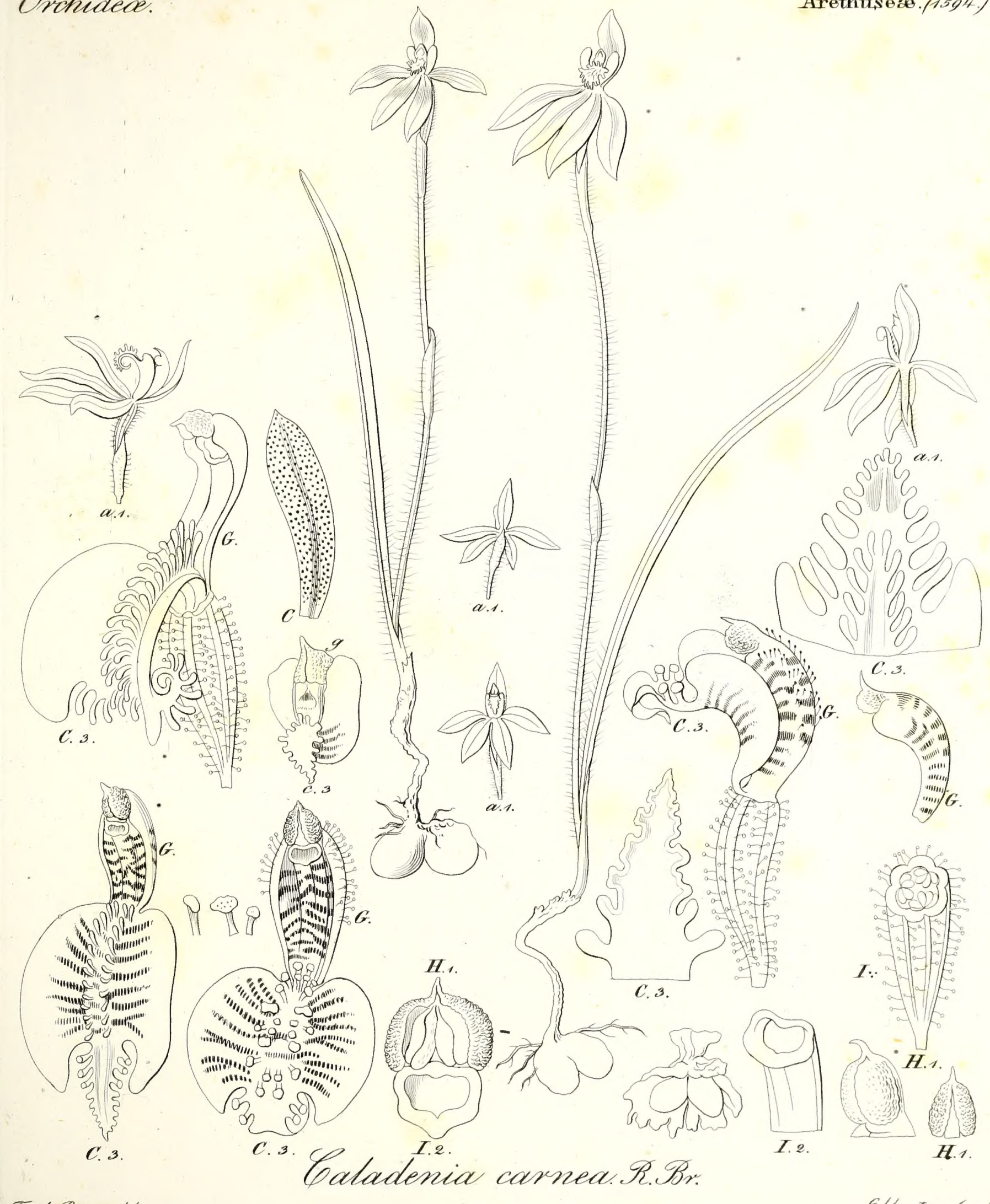



Proteaces.

Franklandiex/2134/

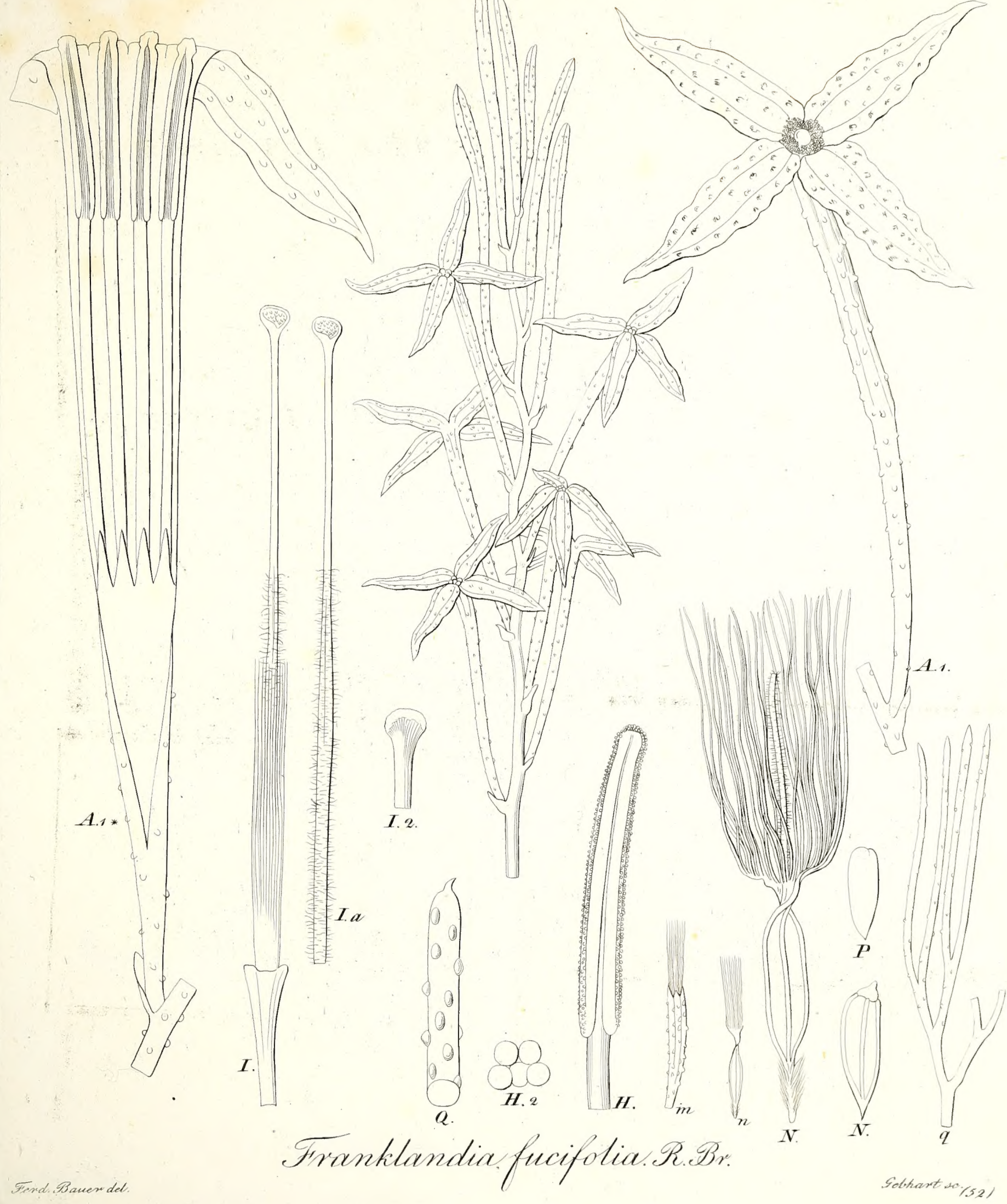



Lobeliacece.

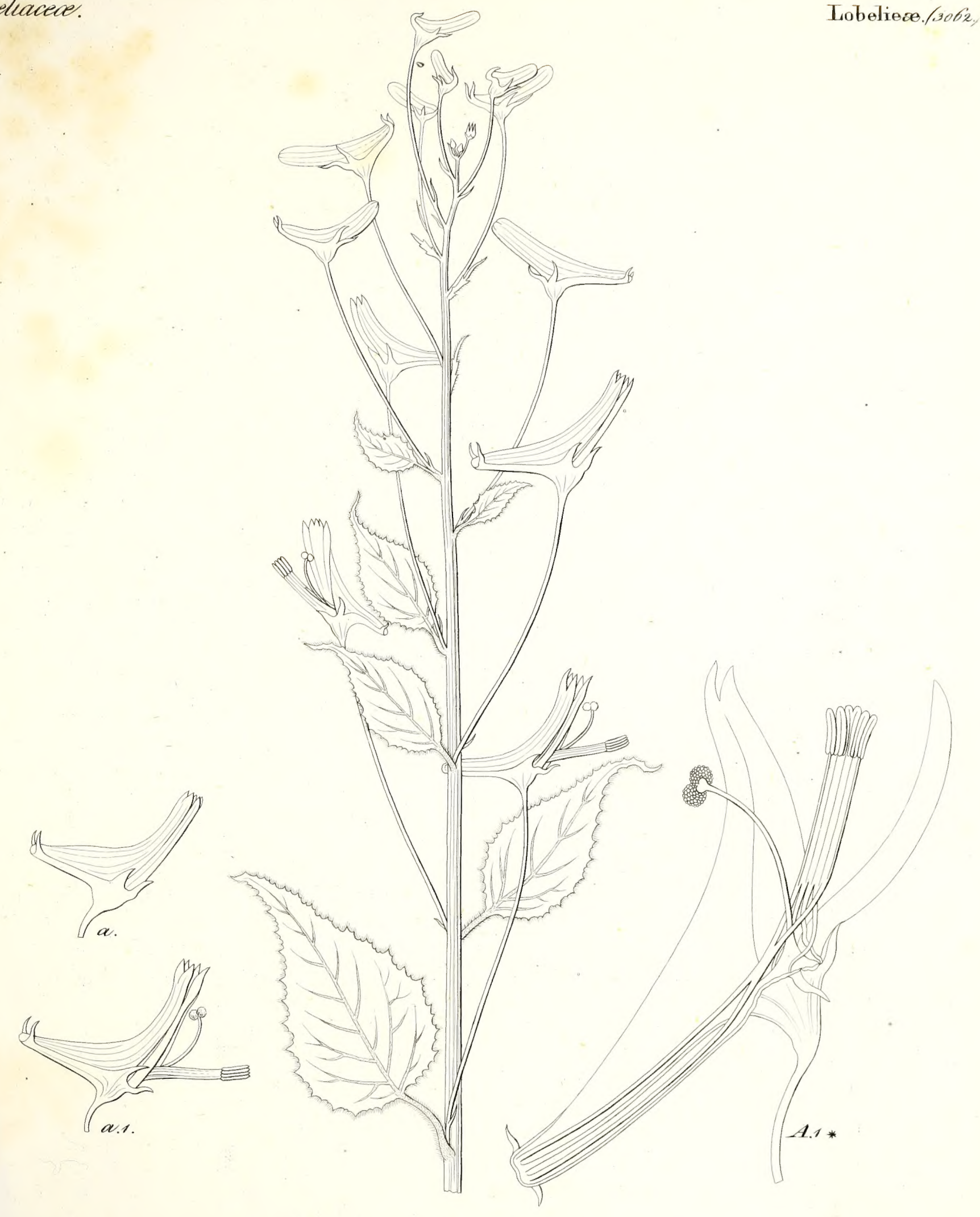

Heterotoma Lobelioides. Zuec. 



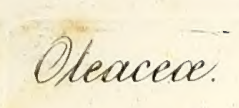

Oleinea(334g.a.)

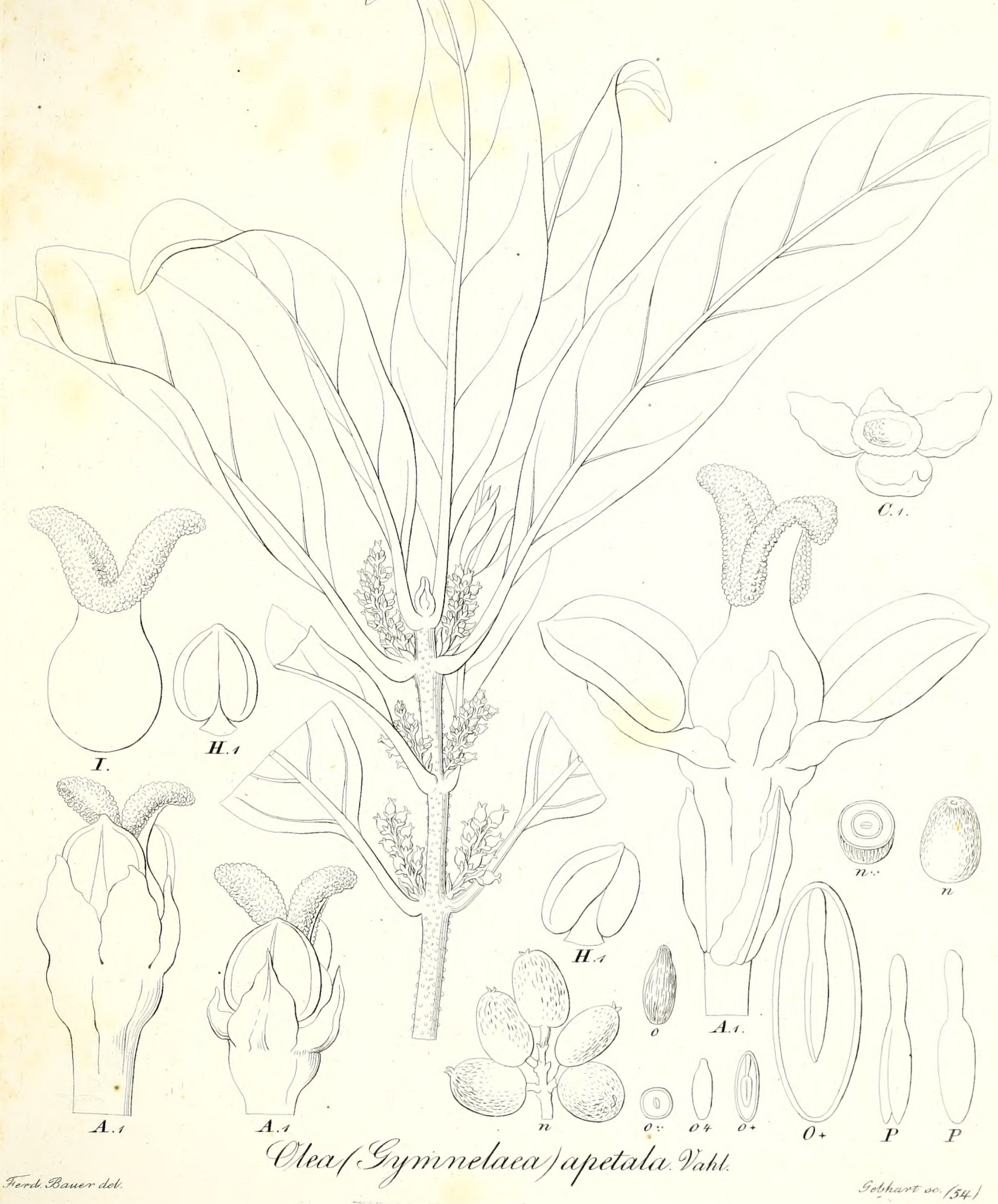



Oleaced.

0leineae/3.350) 

Loganiacea.

Antoniea/3362.
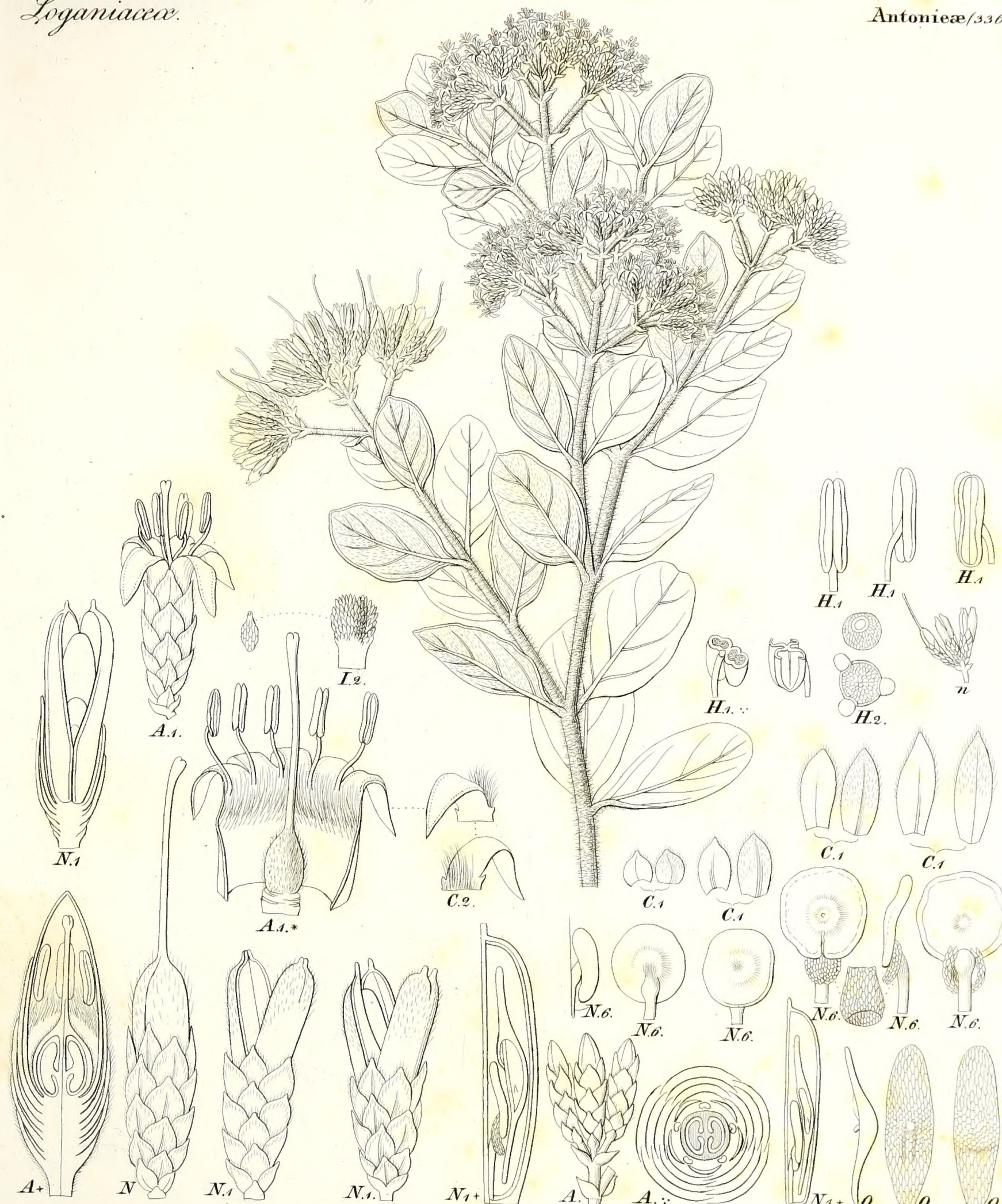

$H_{.1}$ H. 
Loganiacexe.

Euloganieze./3364.6.)

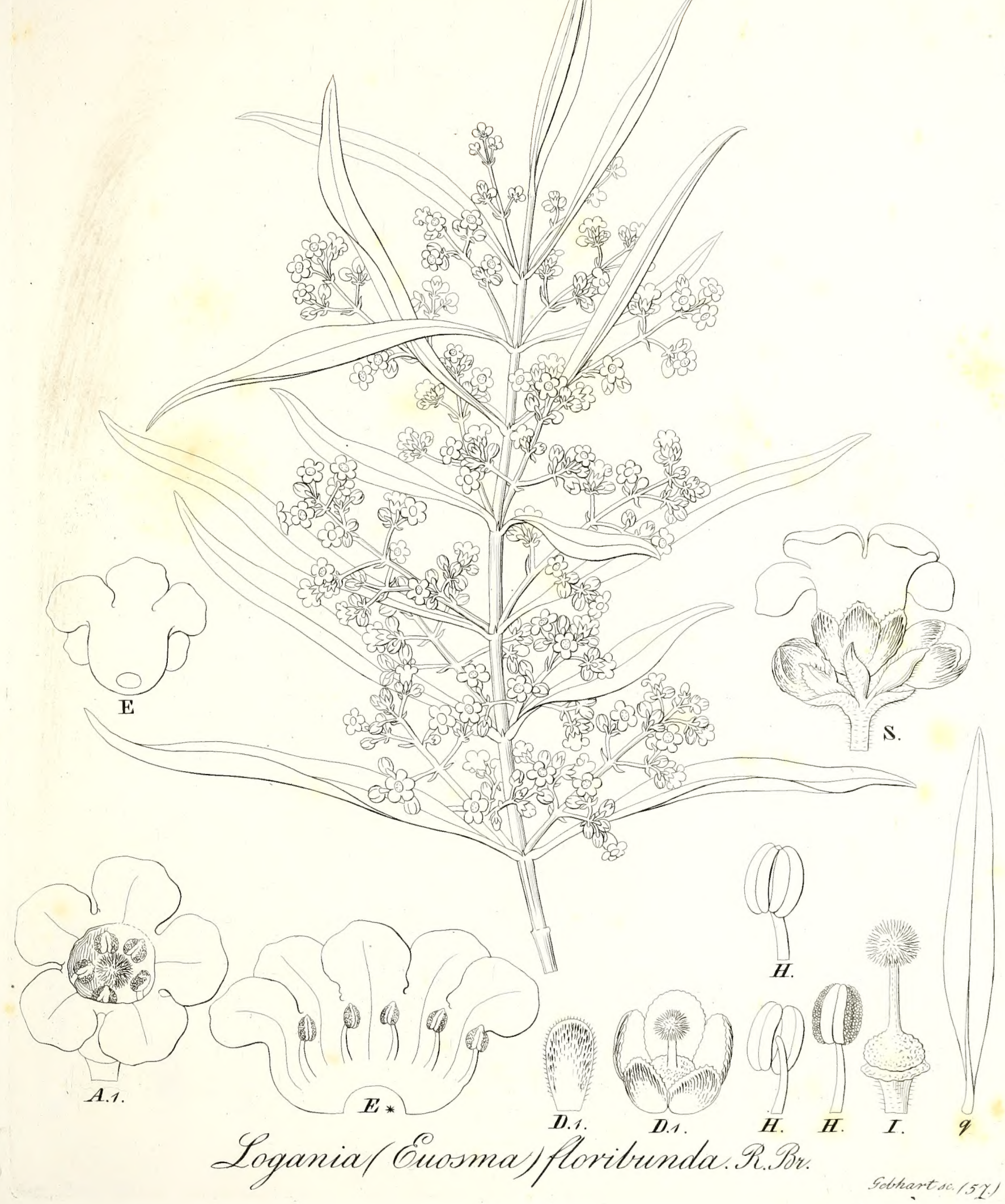


Loganiacea.

Euloganiex/3364. of
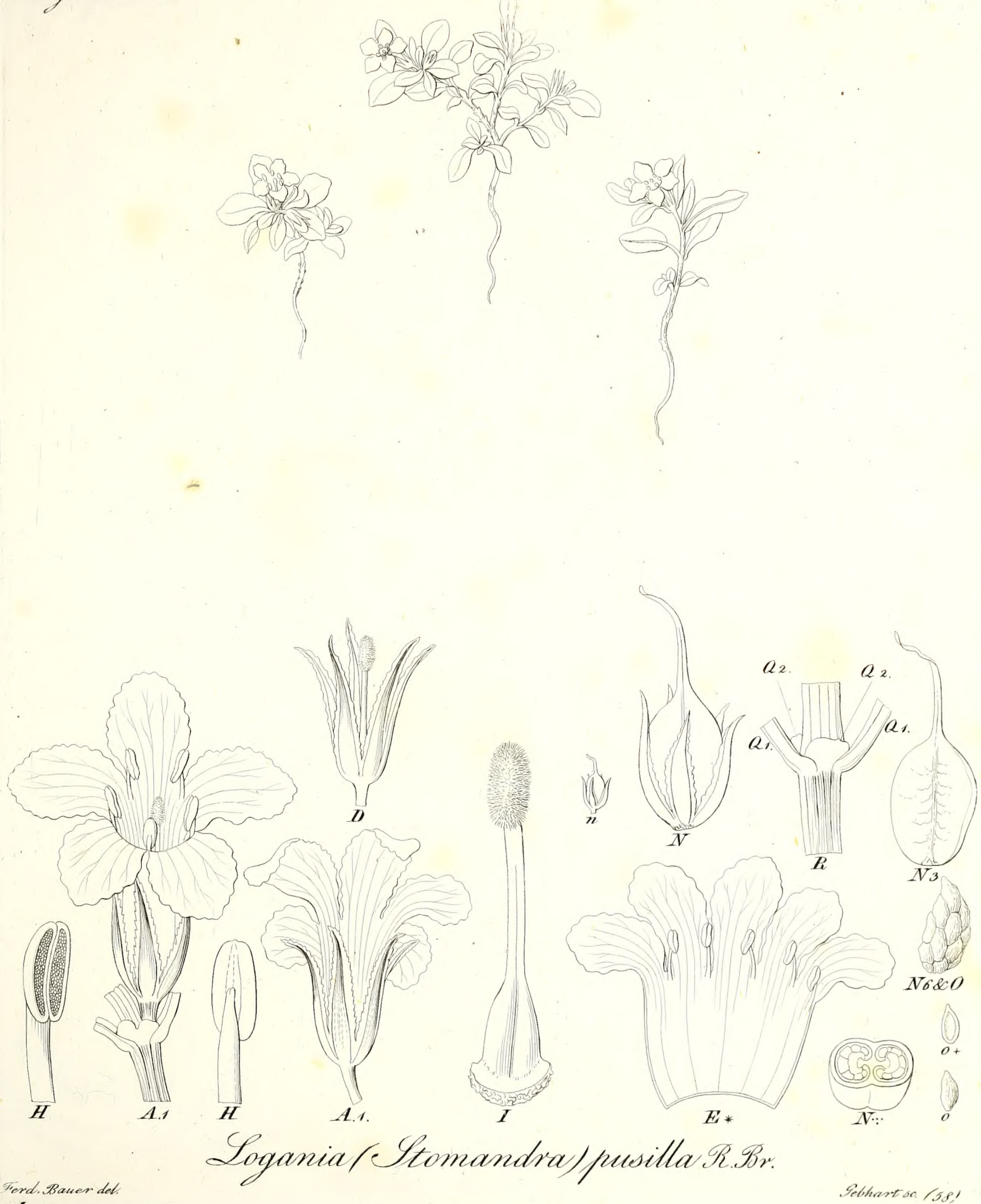


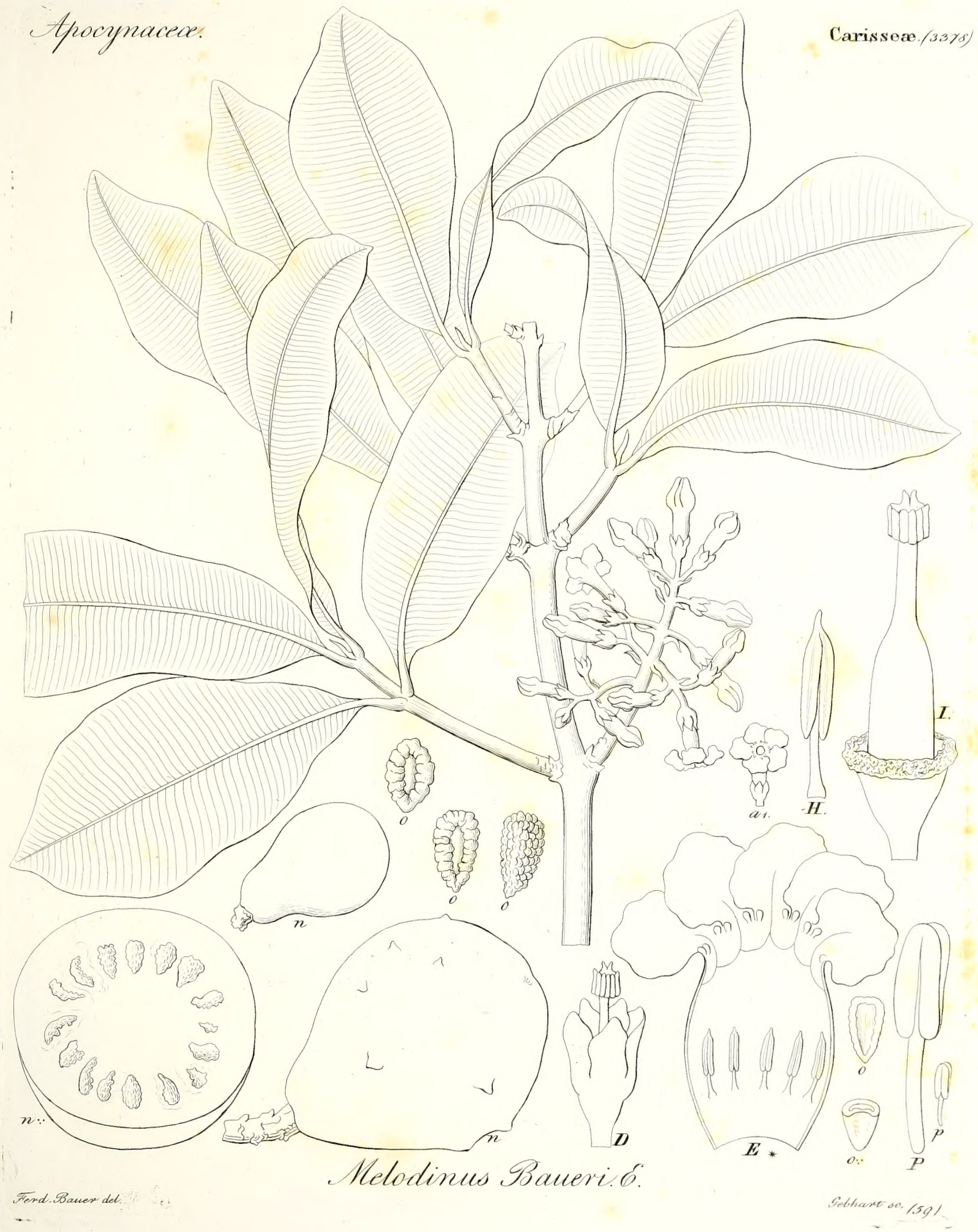



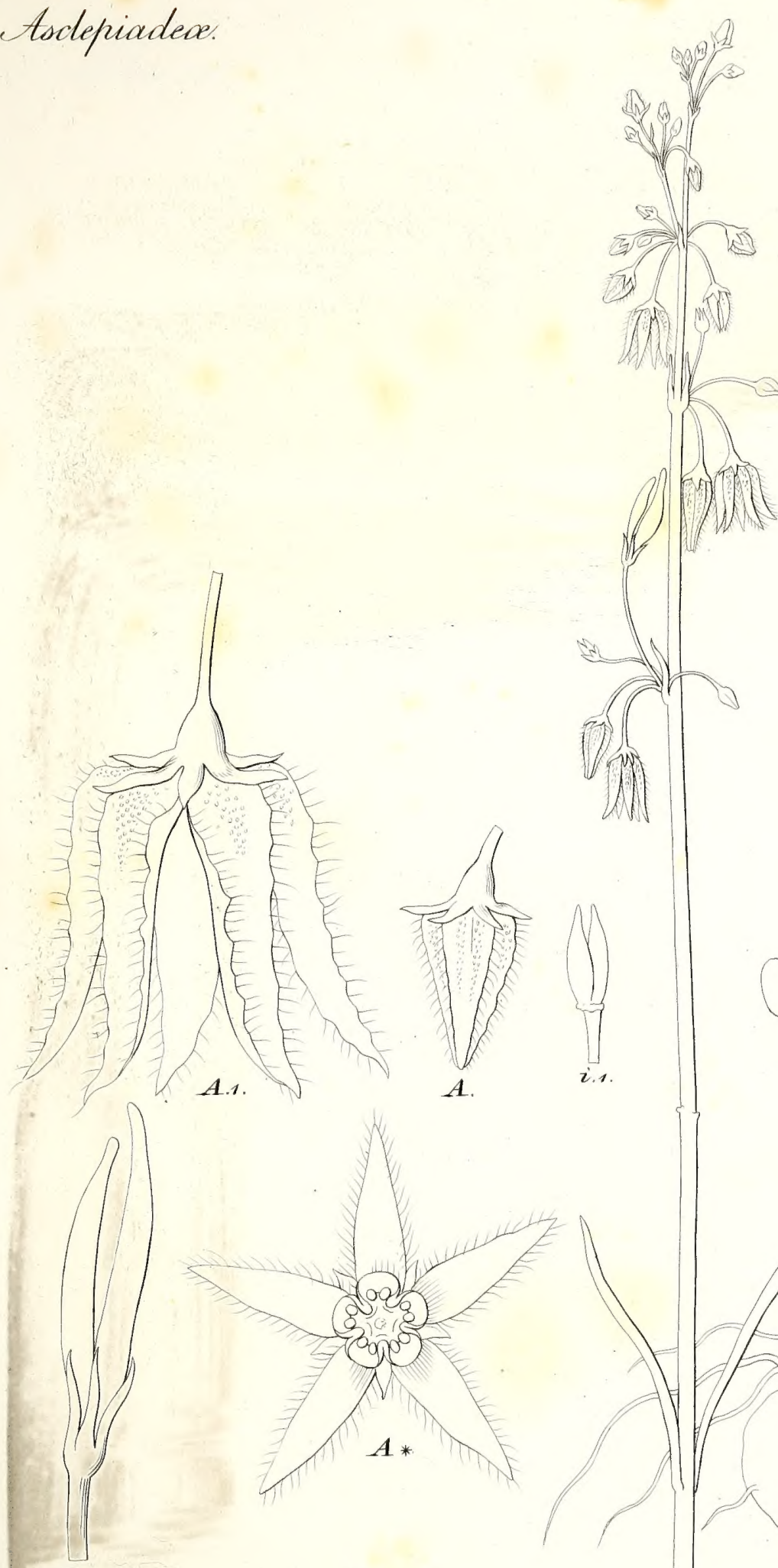

L.1.
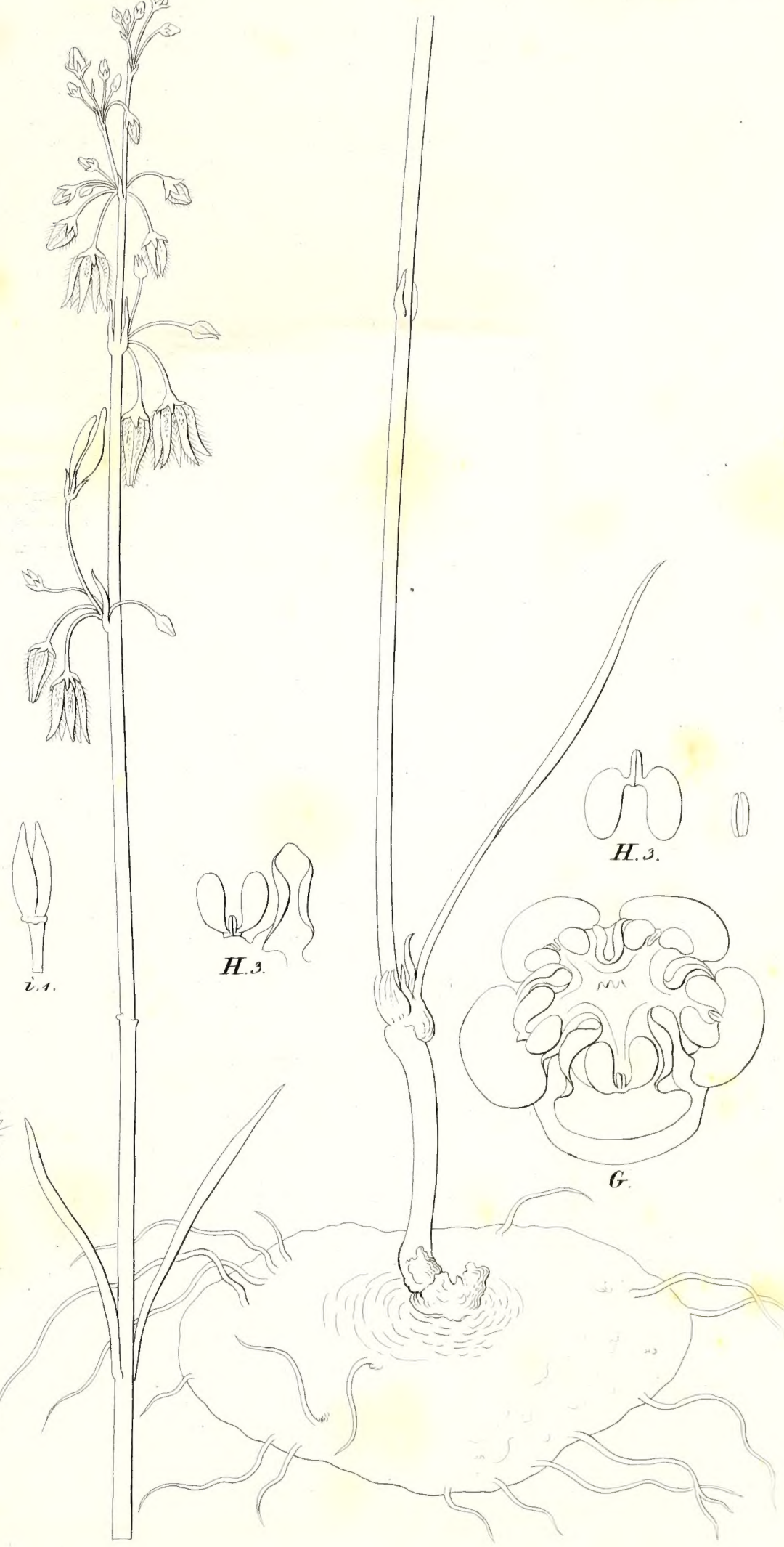

Nicrostemmatuberosum R. Rr. 


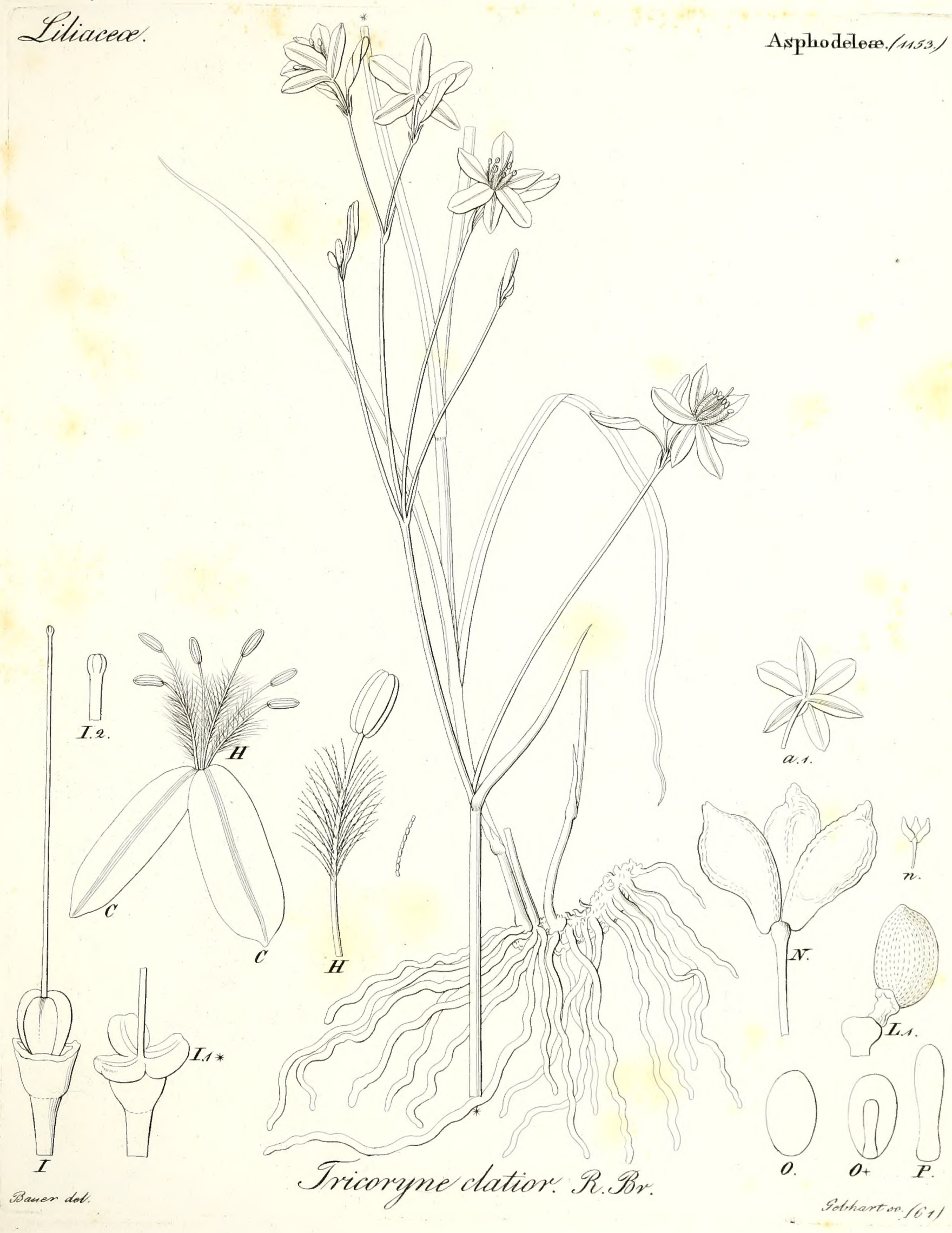



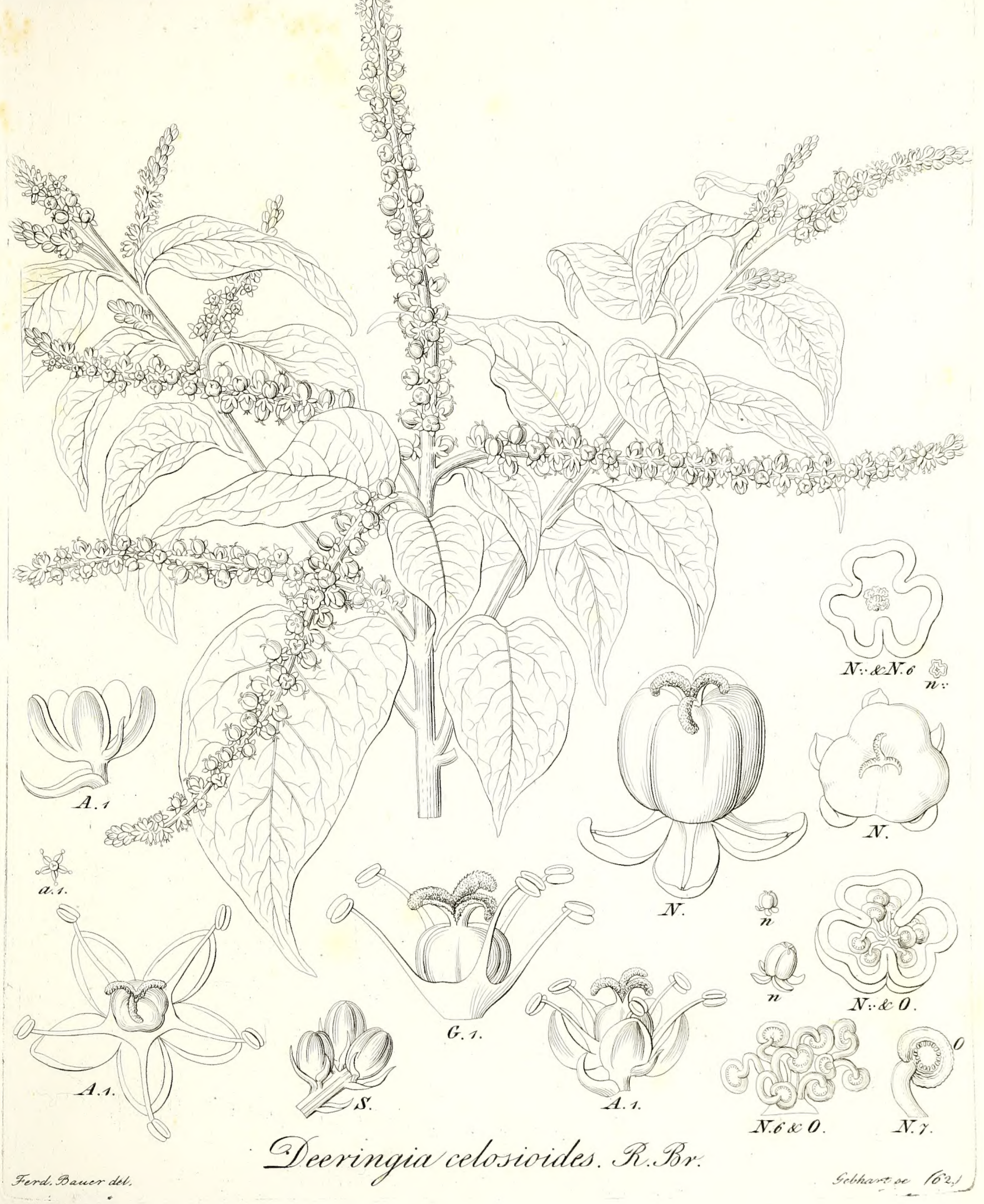



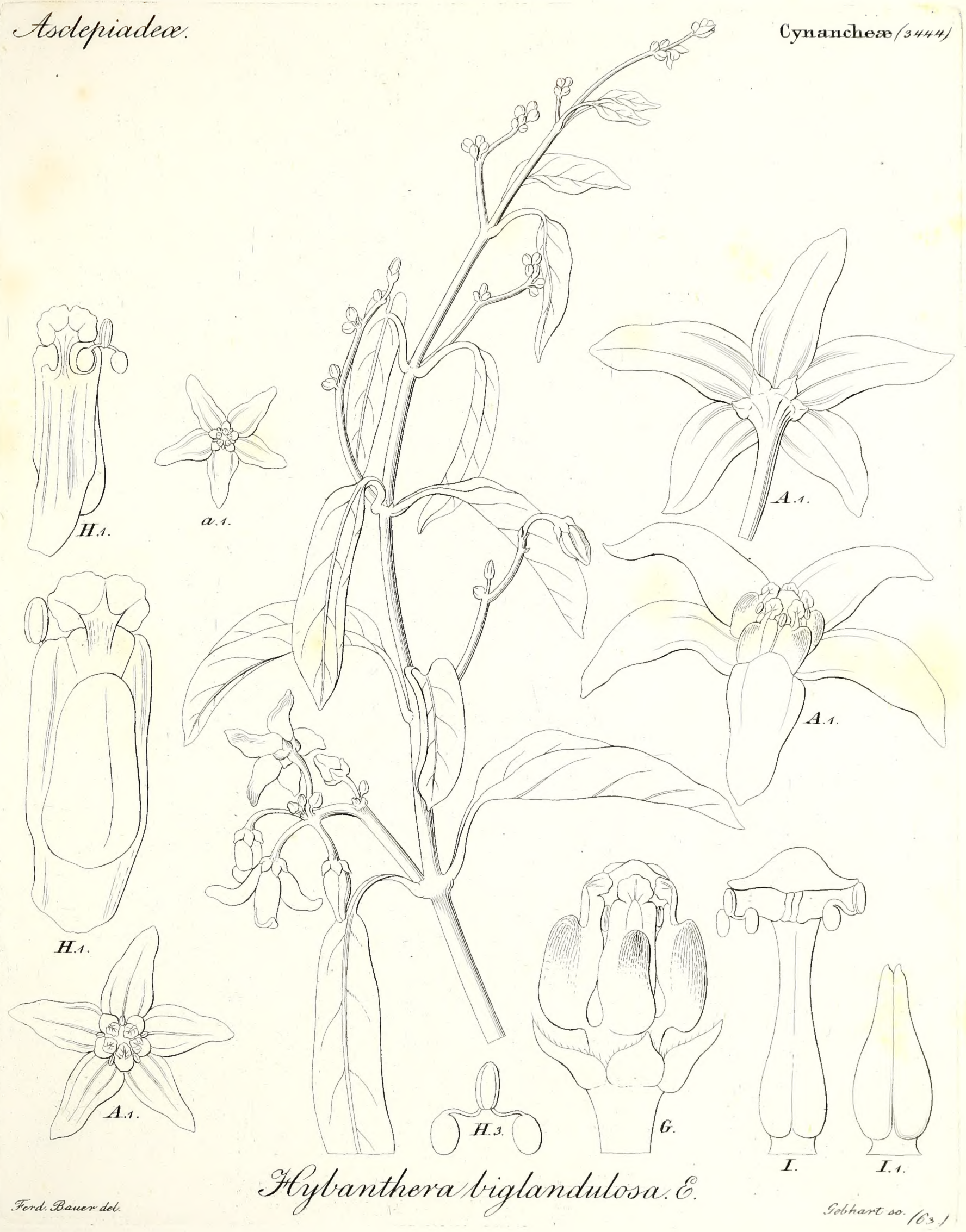





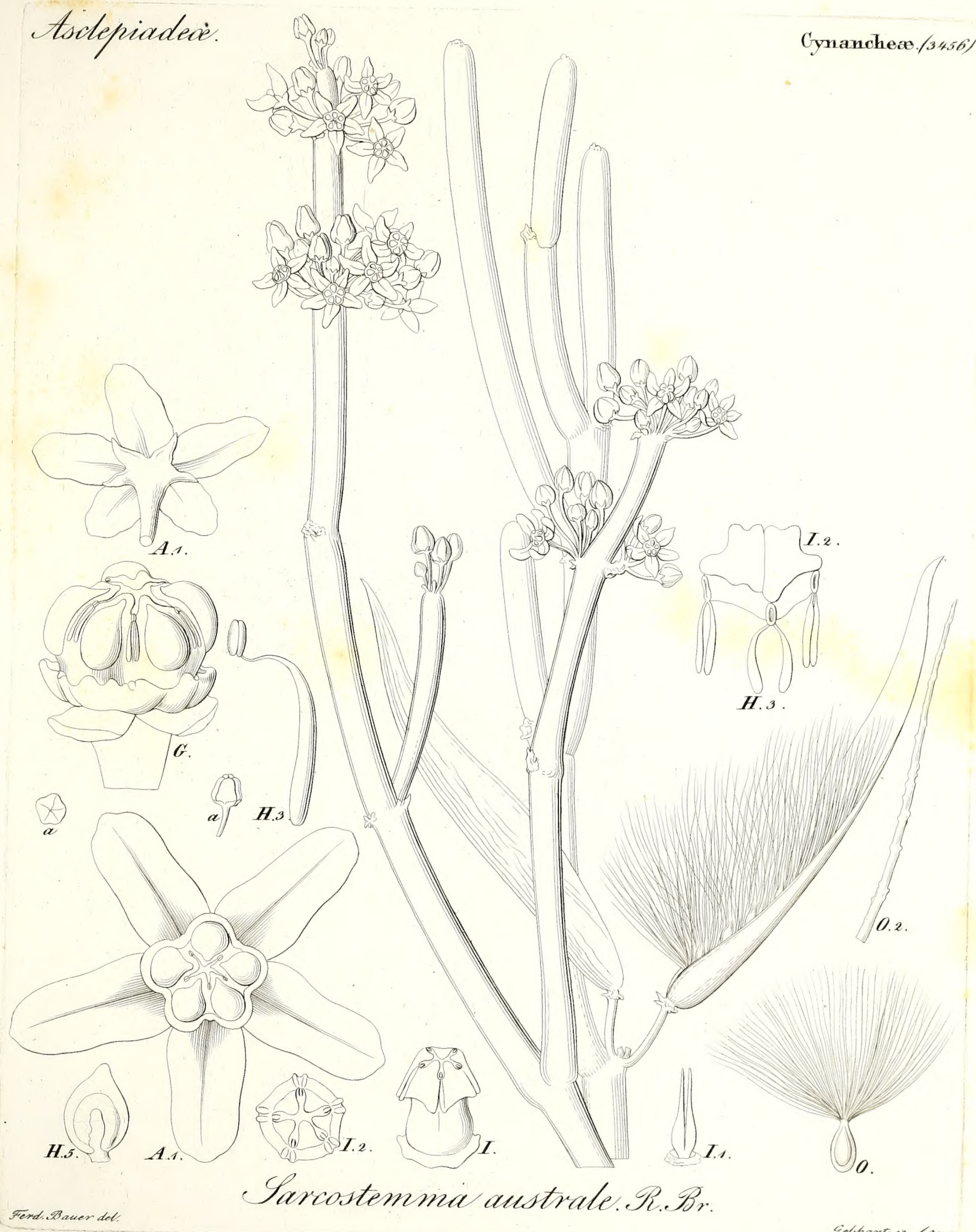




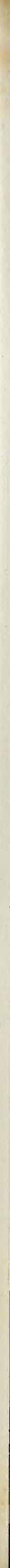




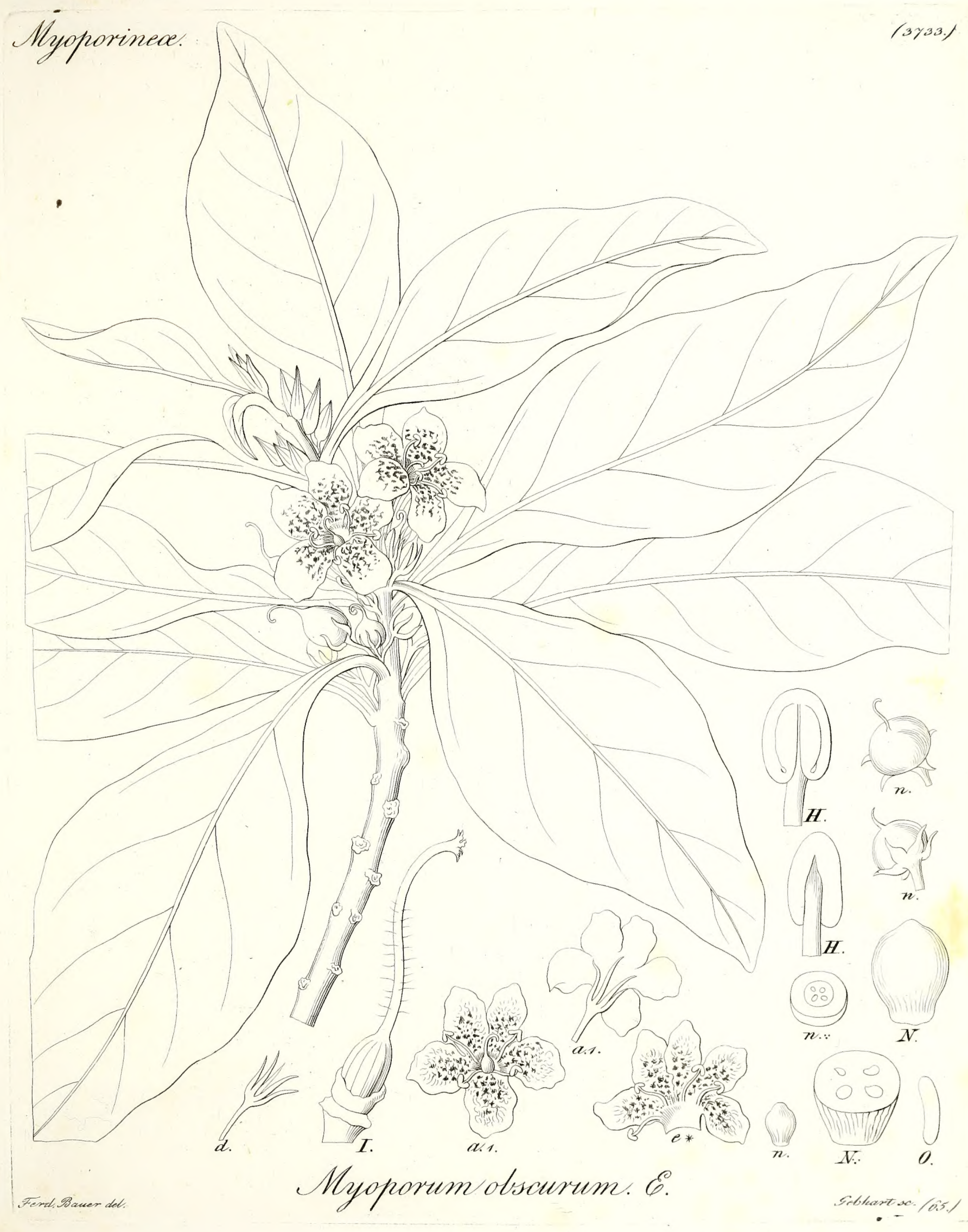





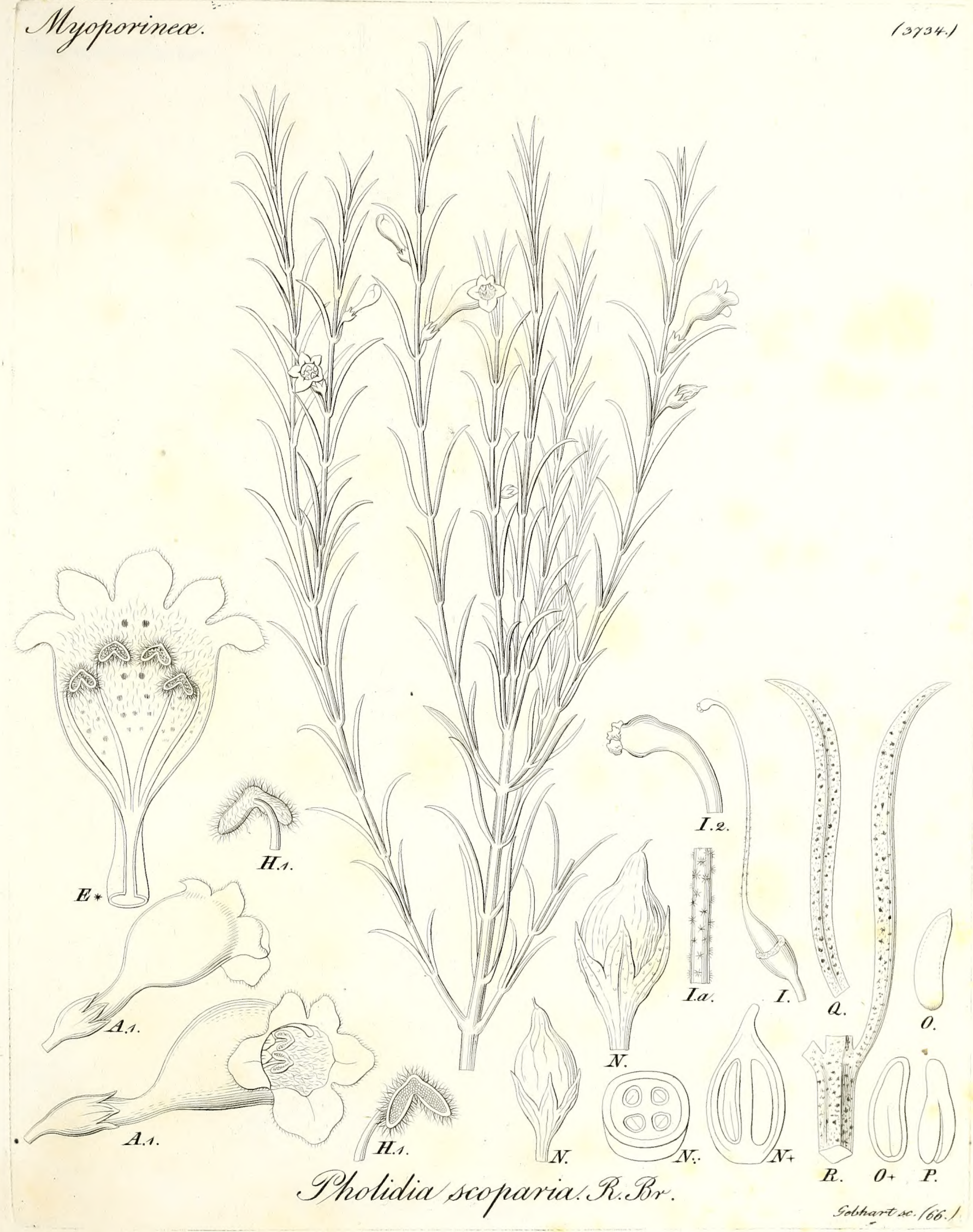


Gomvolvulaceae.

Convolvuleas ( 3800.$)$

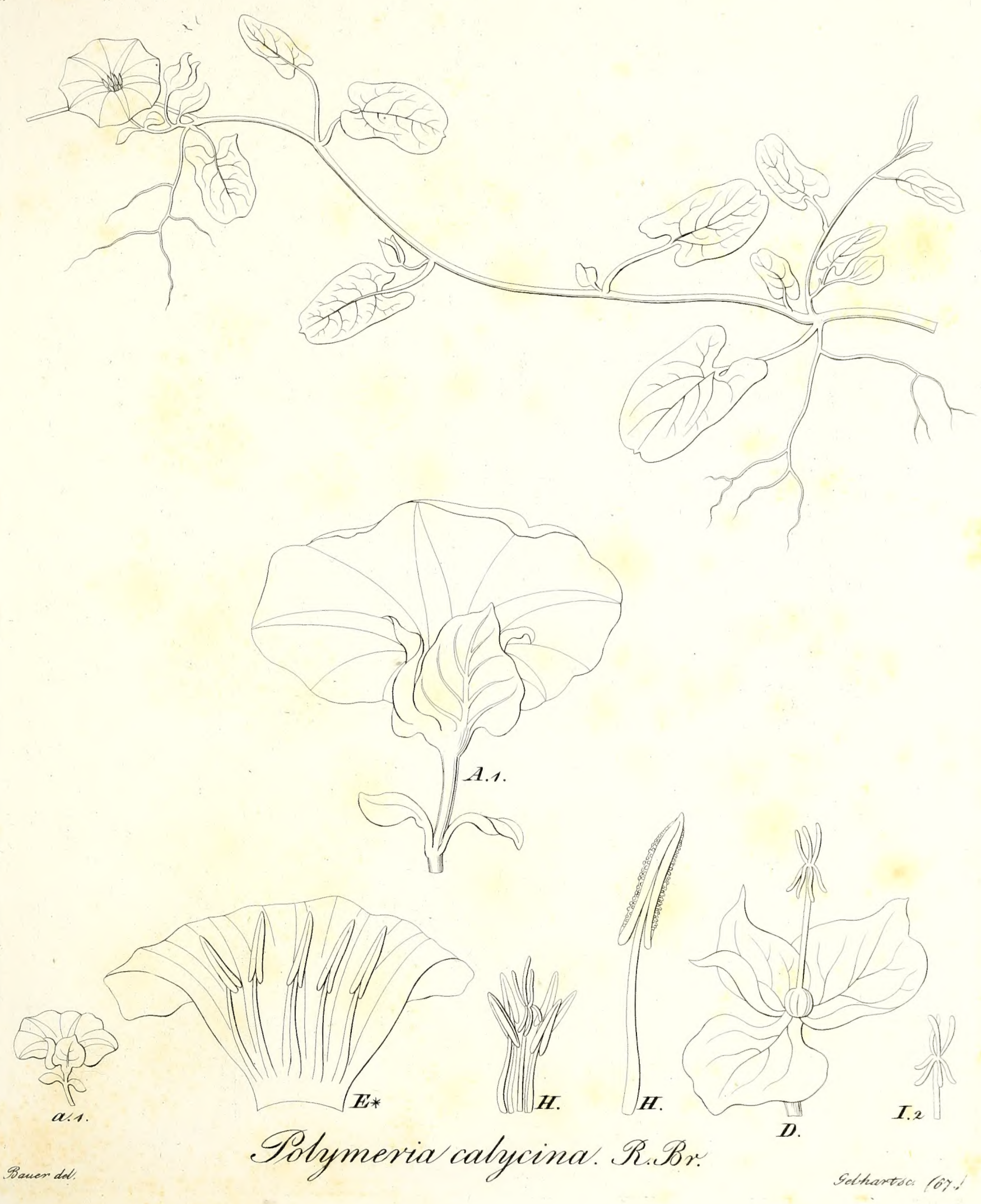





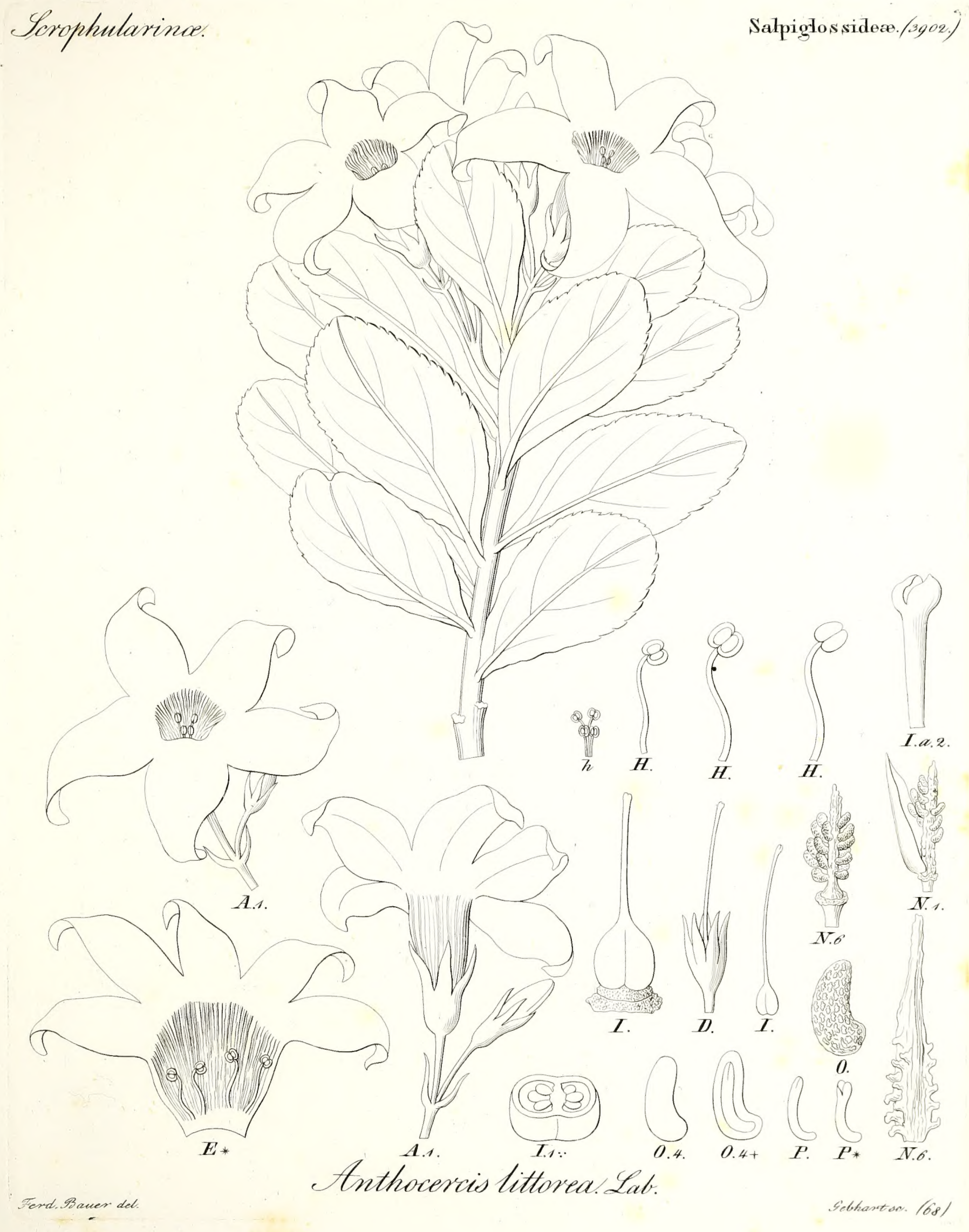





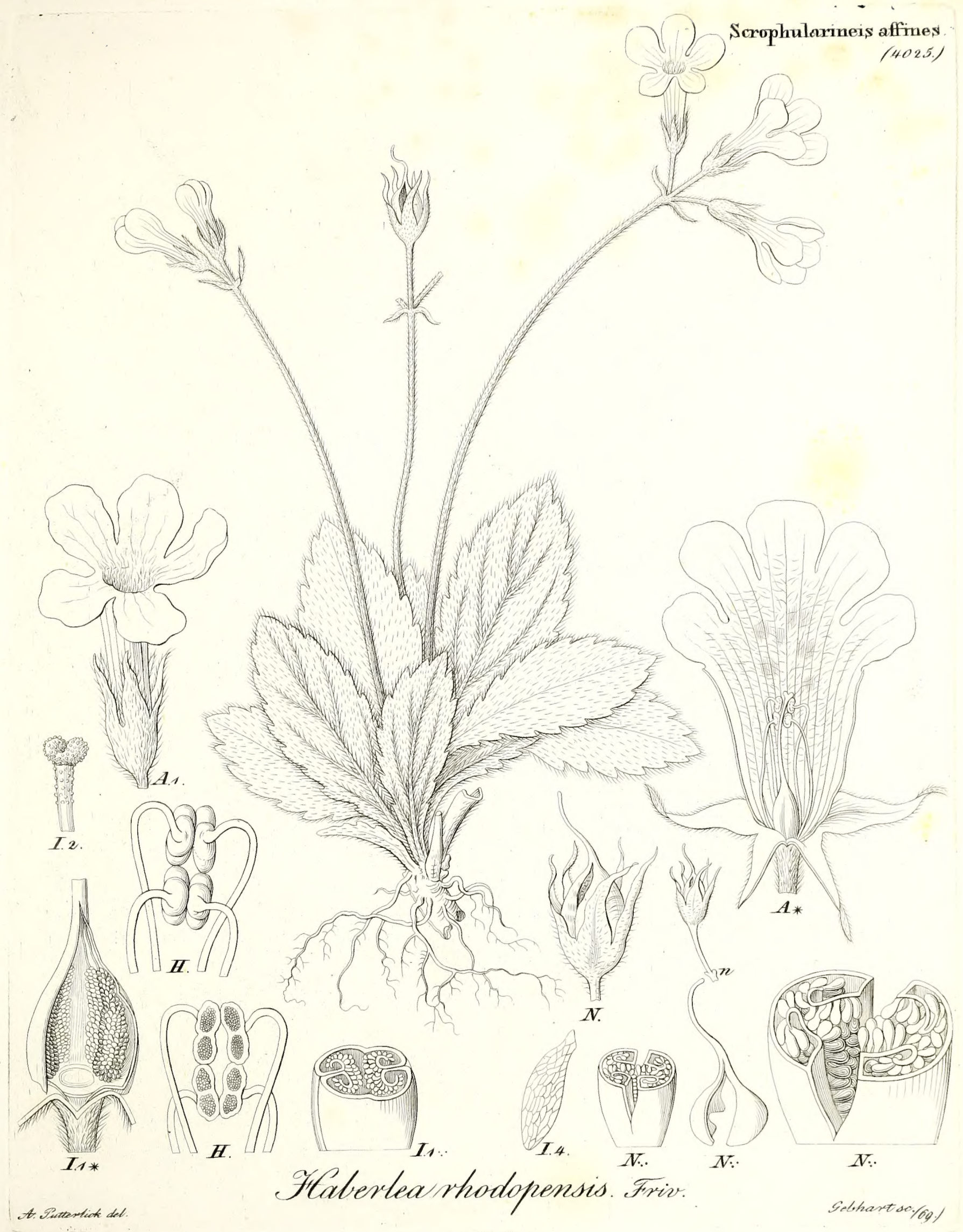





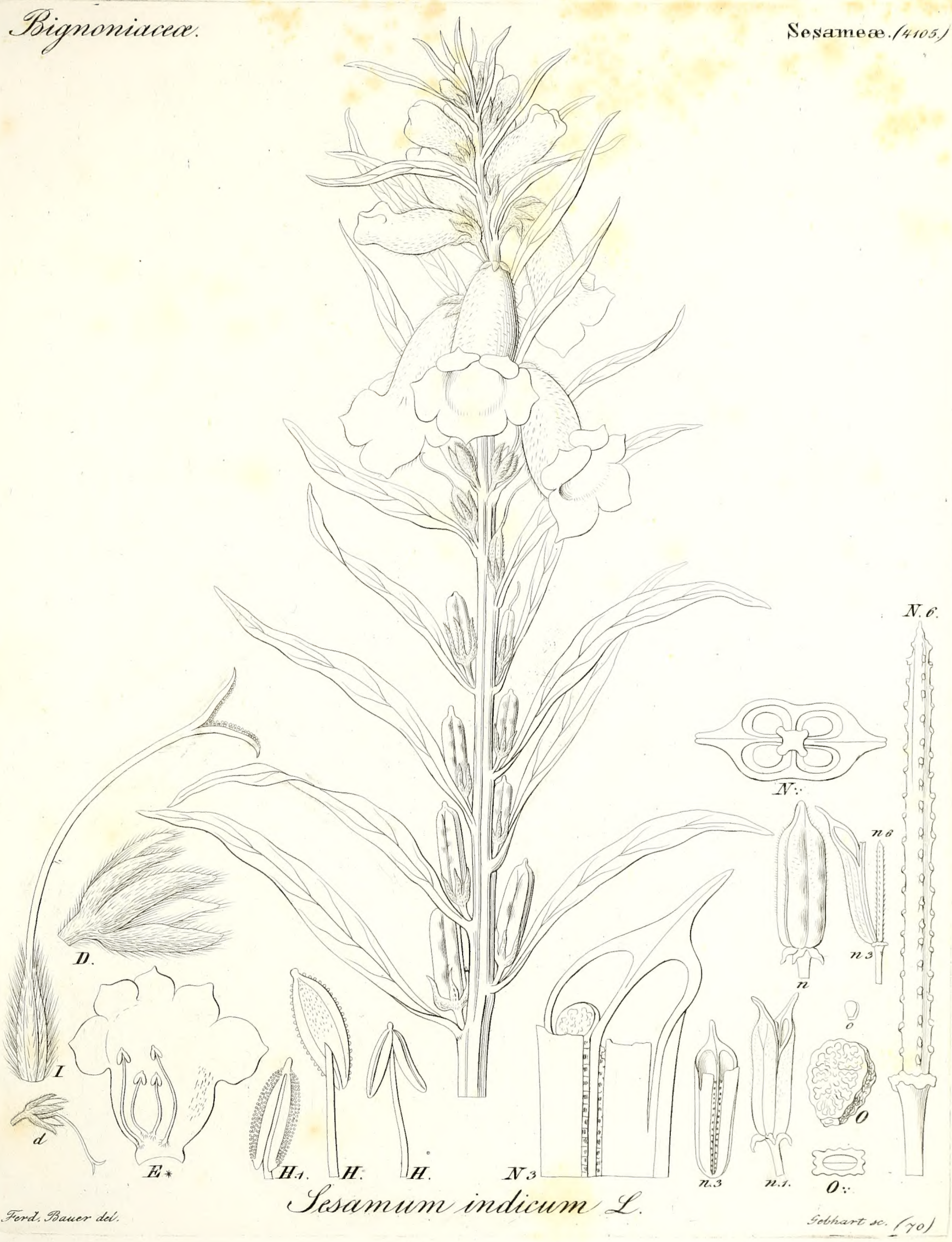



Bignoniaced.

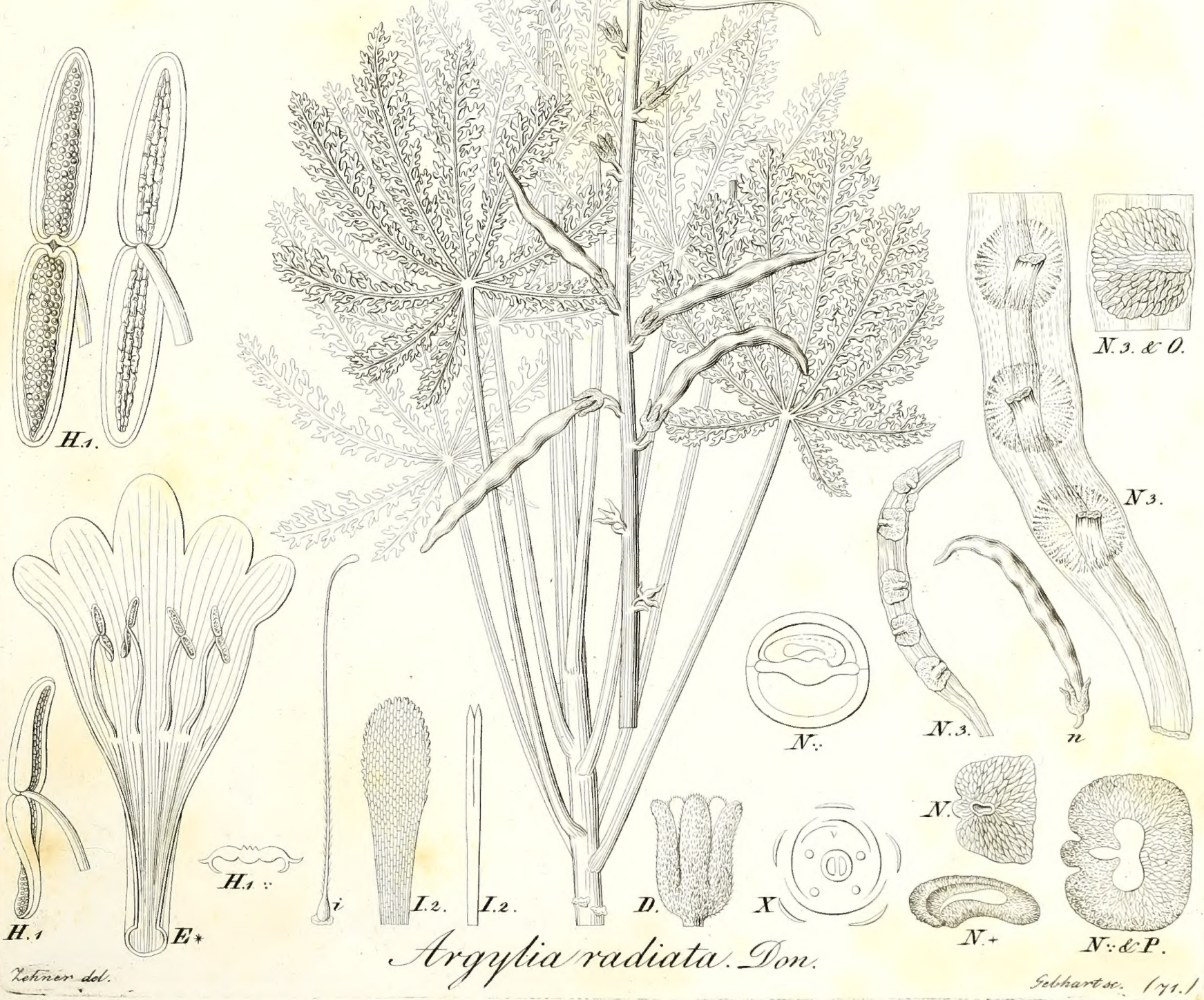




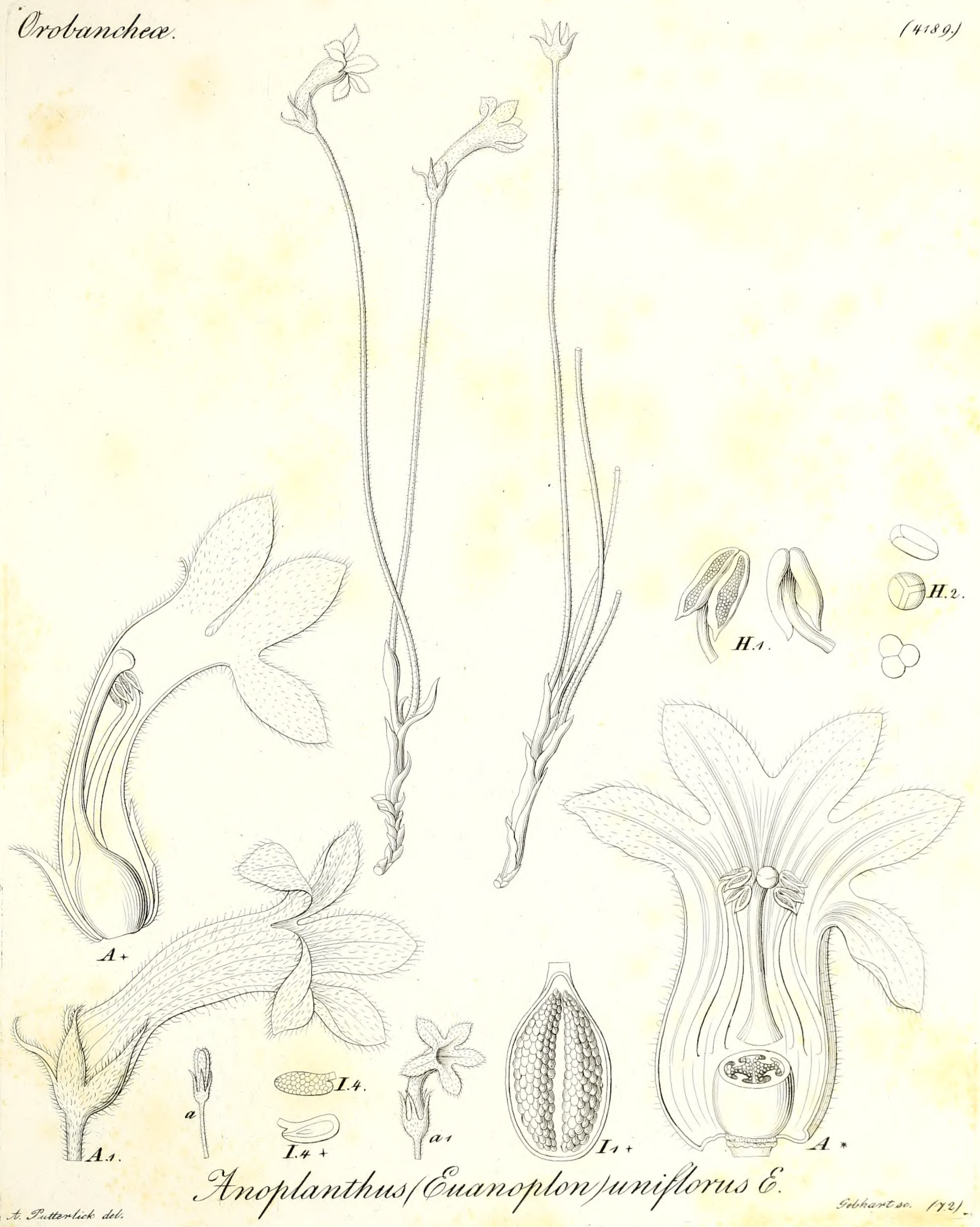



Najadese.

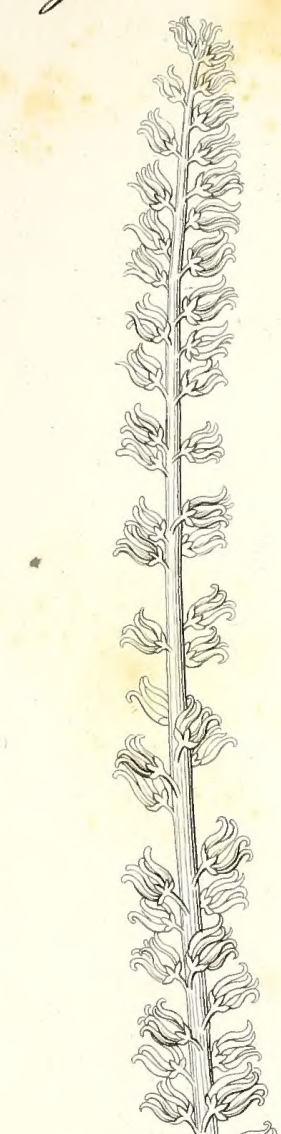

हका है। है।
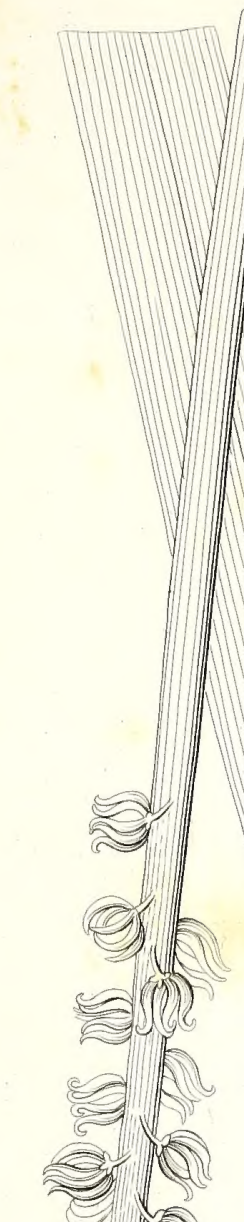

m.
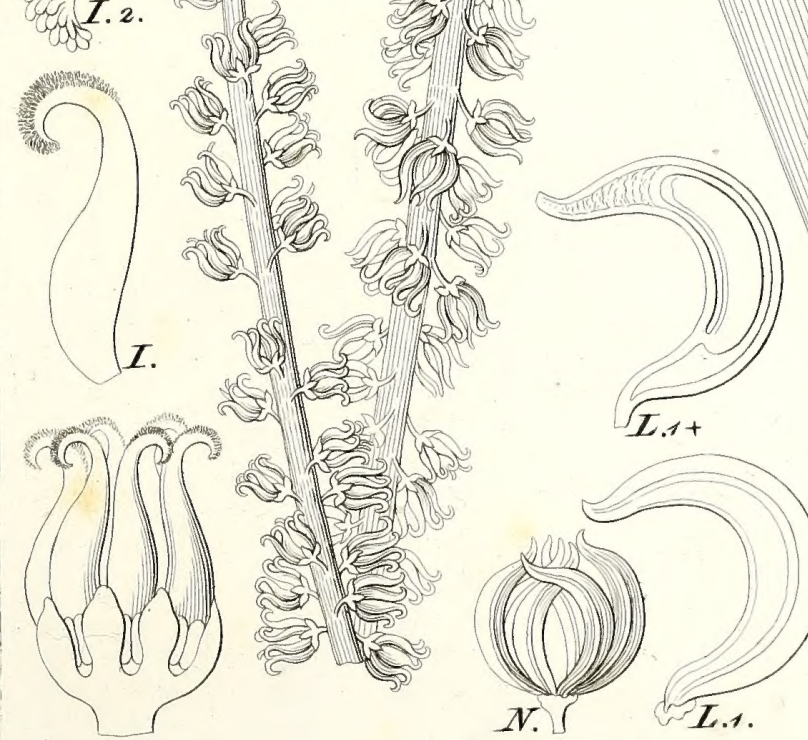

A.1.

M. Quner del

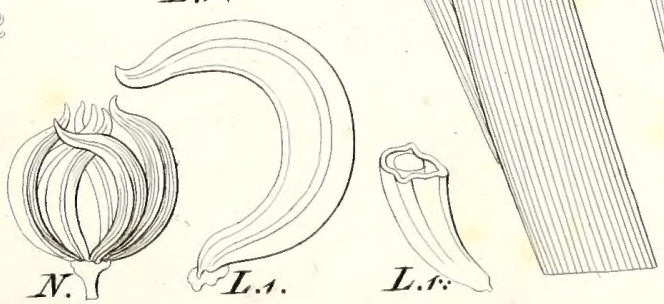

Gyenogeton Ftügelii. E.
(1664.)

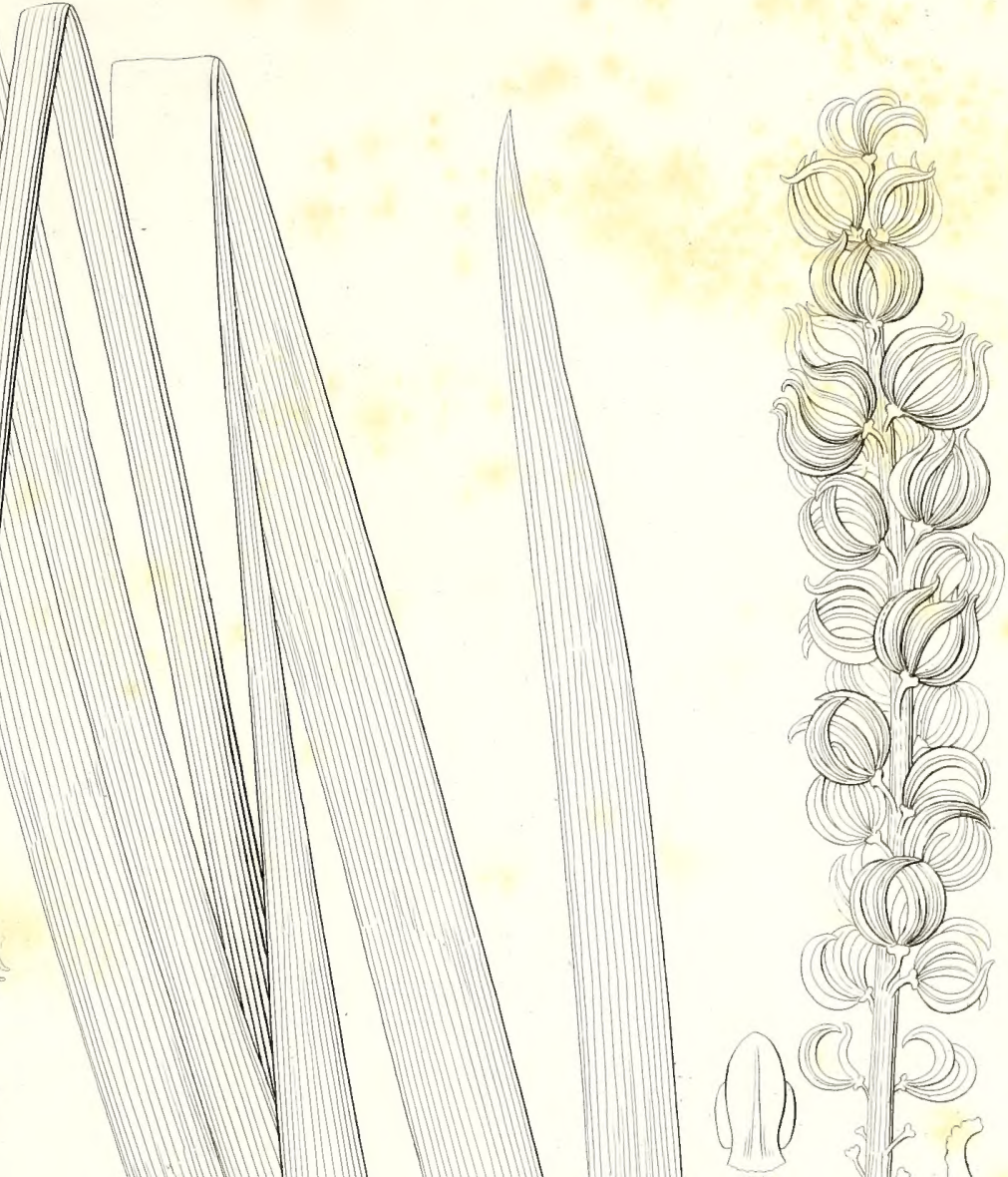

H.

(2)

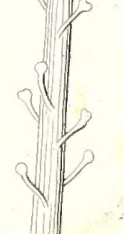

H.

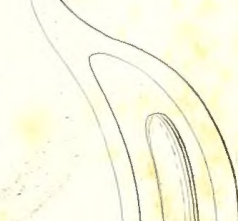

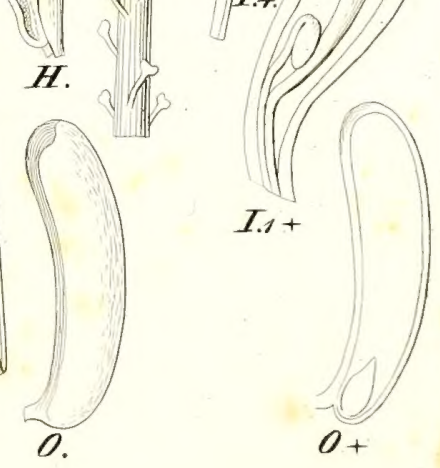

Sebhart so. (73) 


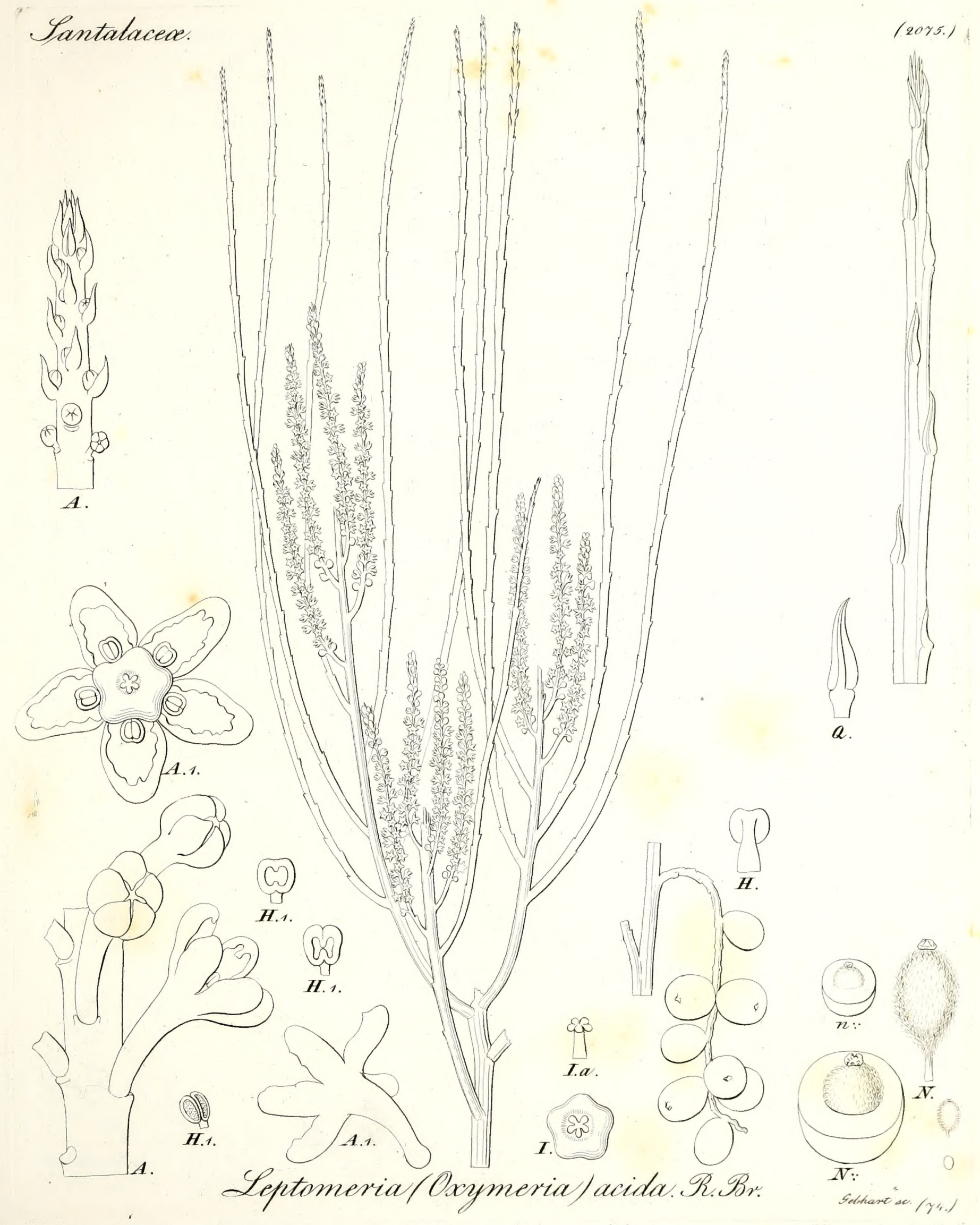




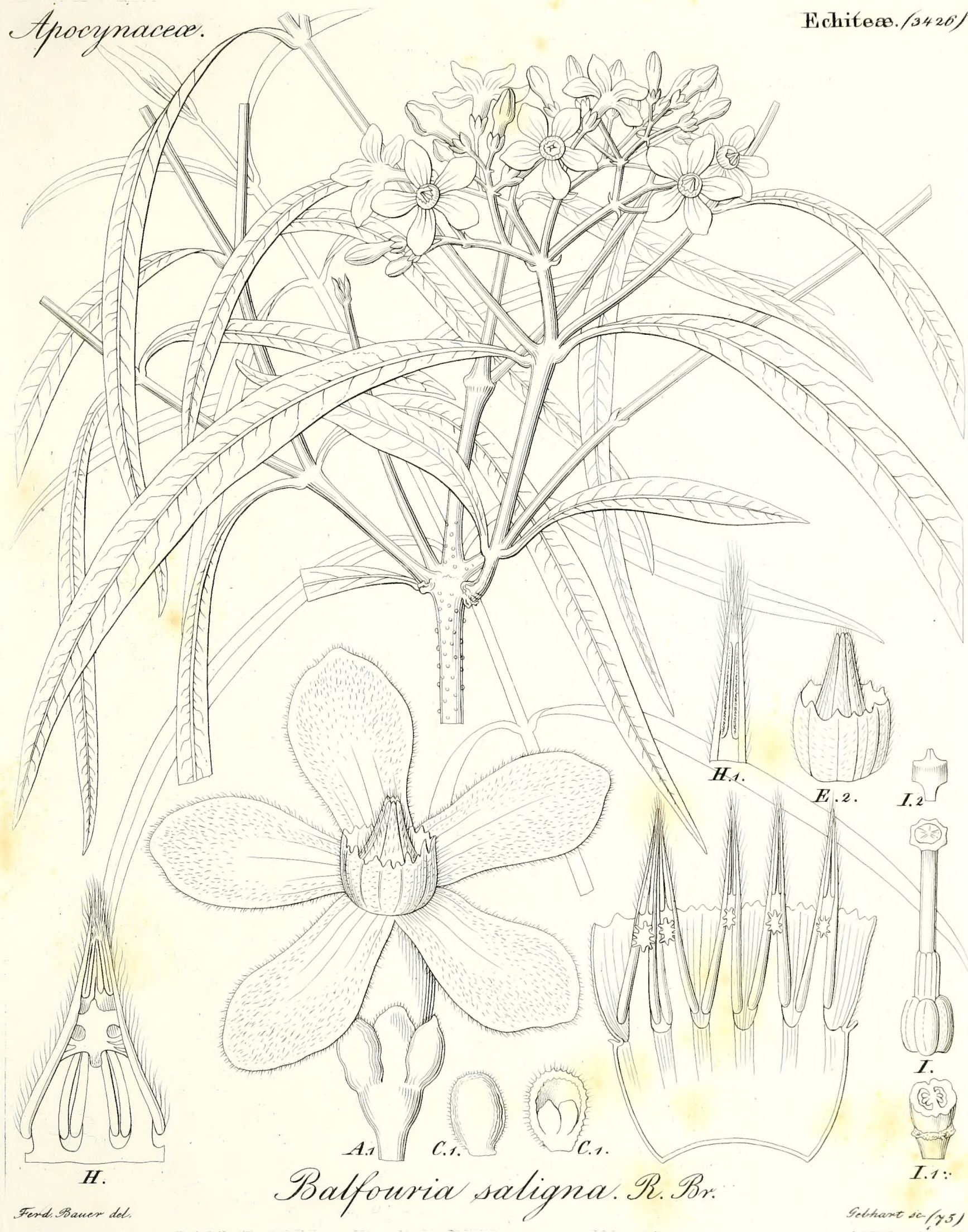


Selaginea.

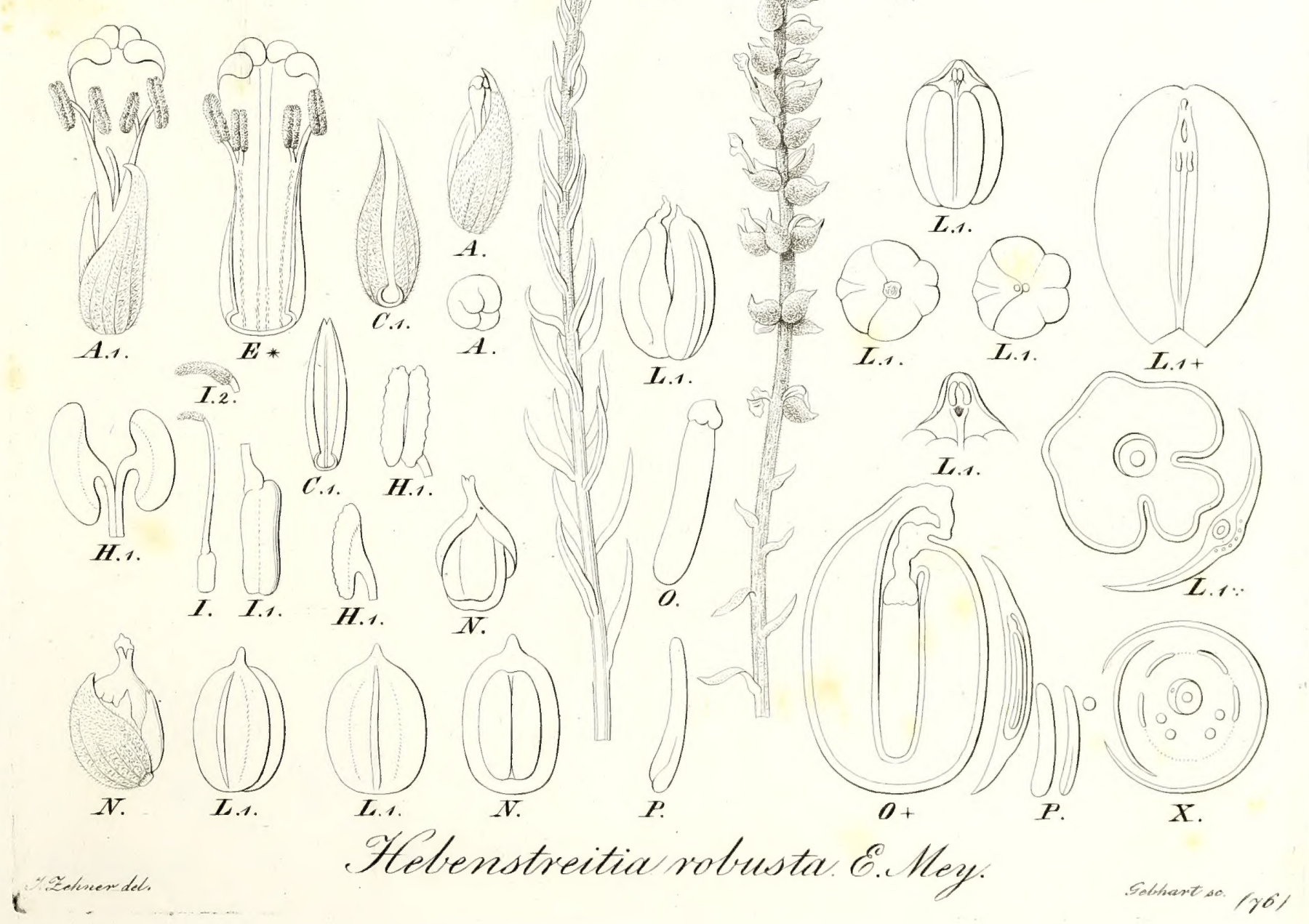




\section{Serophularineae.}

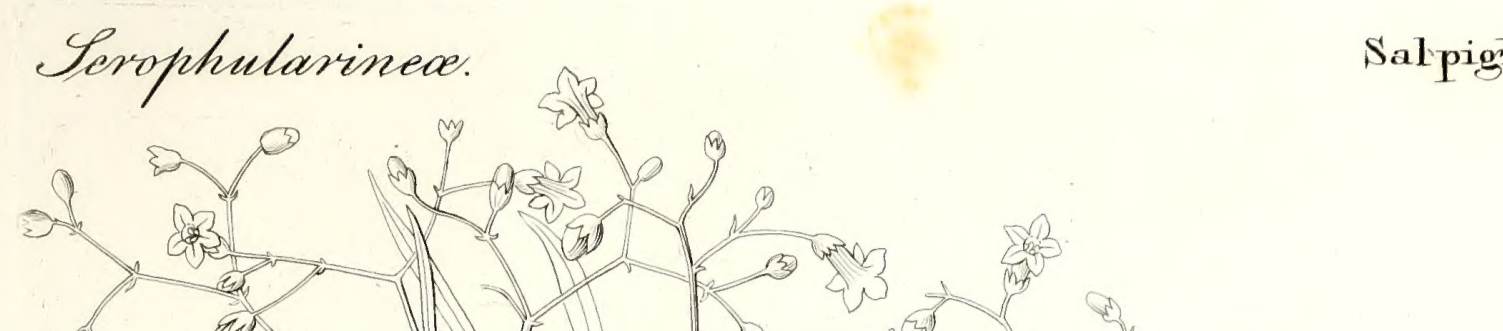

Salpigloffidere(3go6)
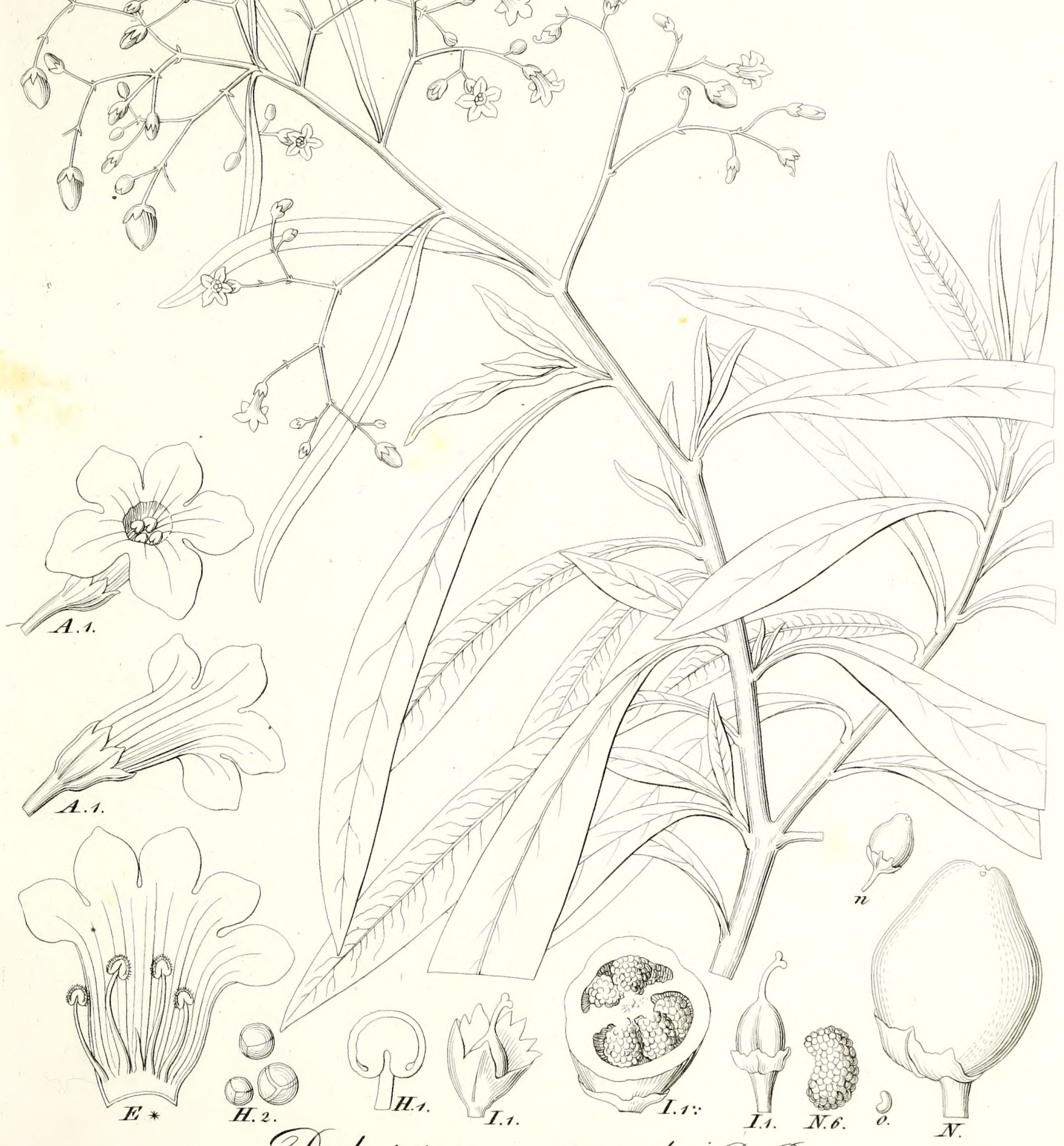

Ford. Bauen del,

Oubrisia mypporoides: R. $\mathscr{B}$ r. 


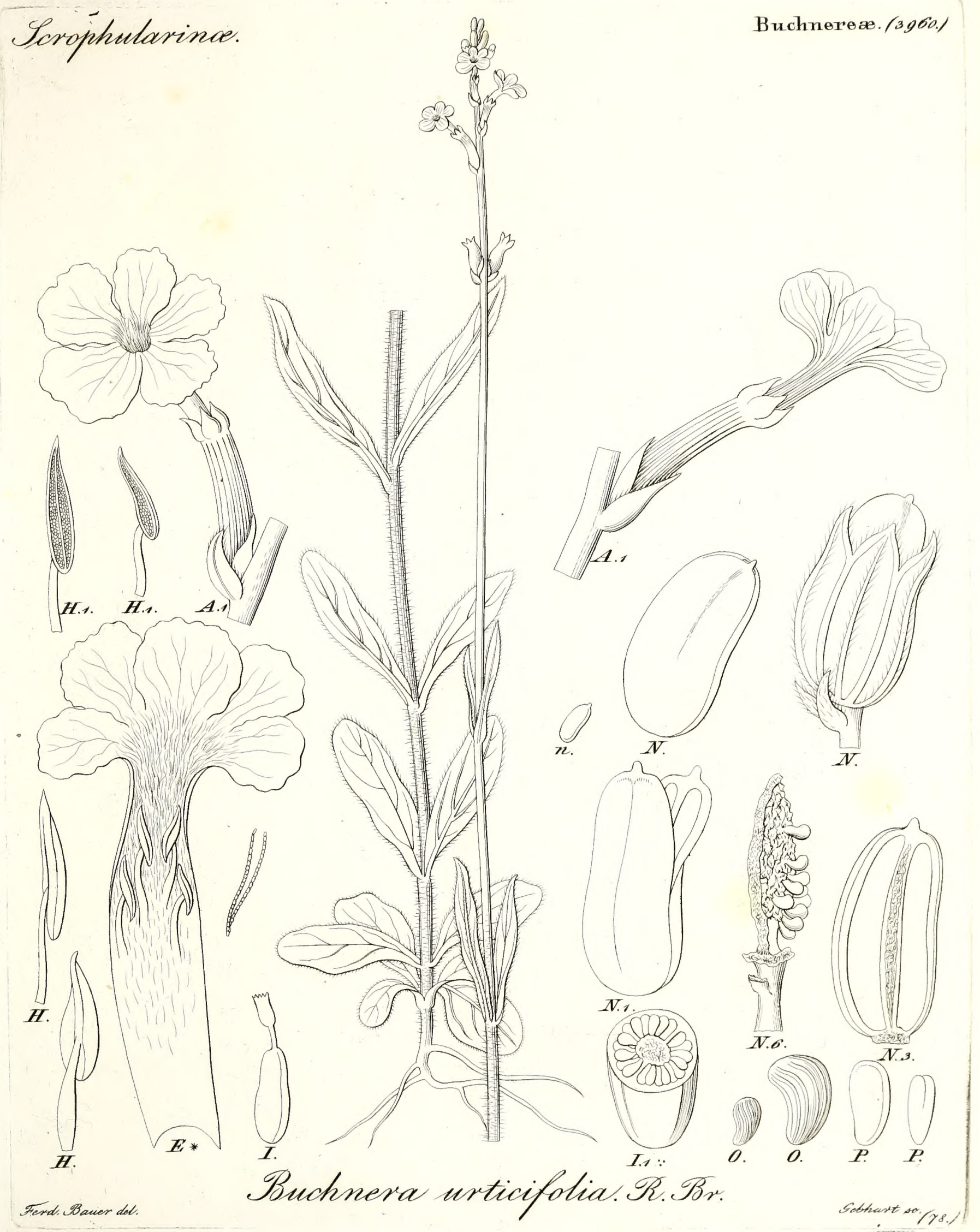




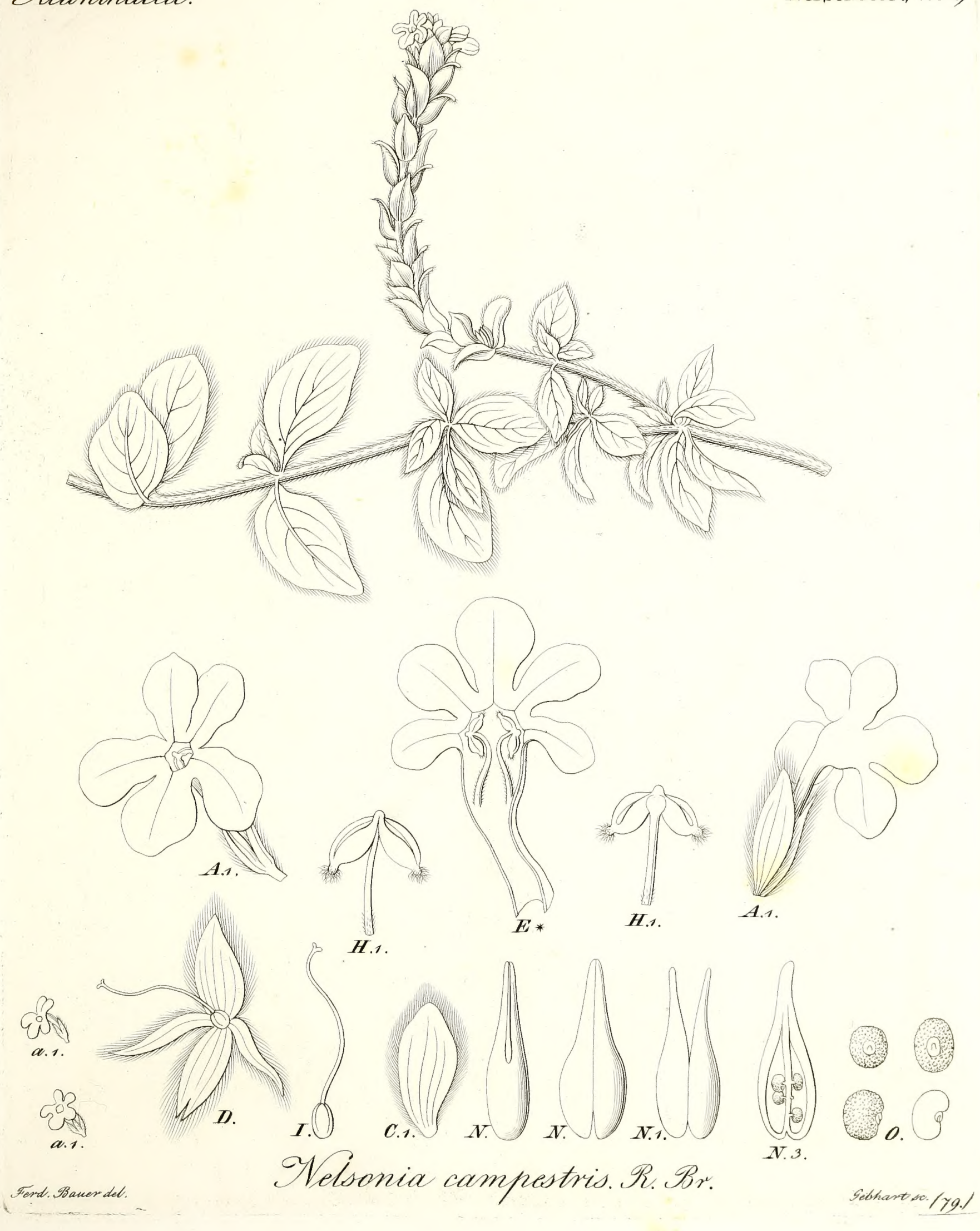


Orobanchea.
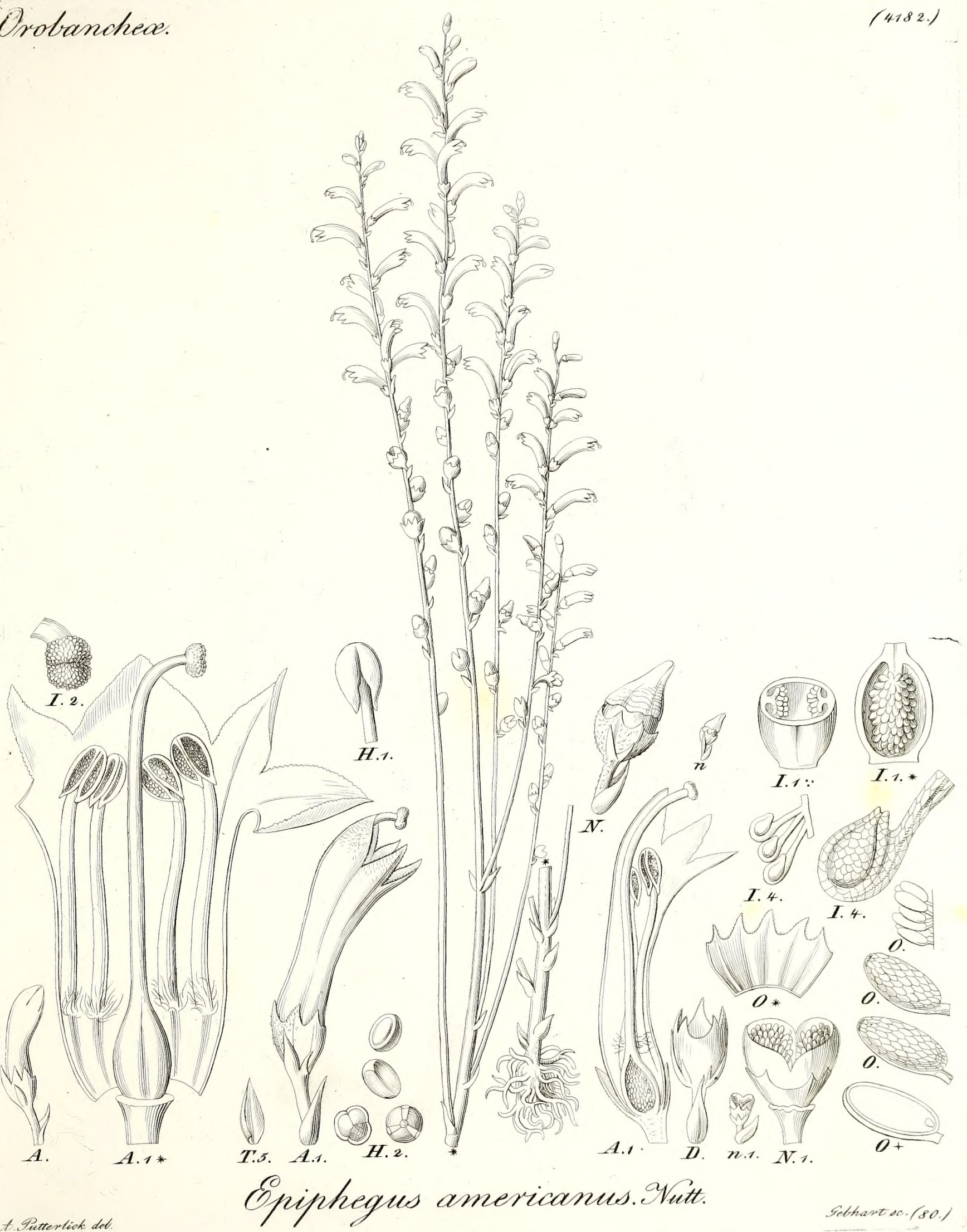

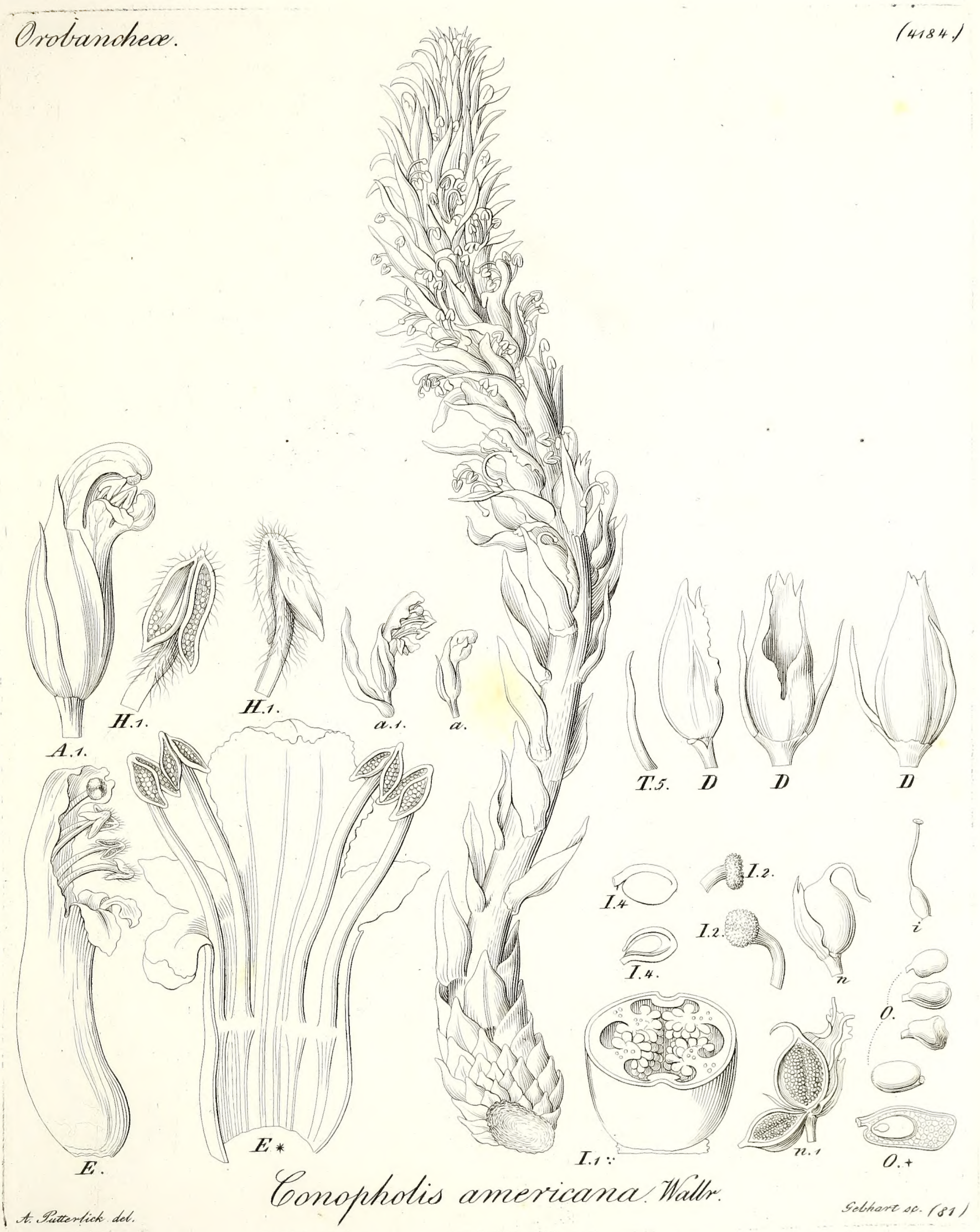
Orobanchea.
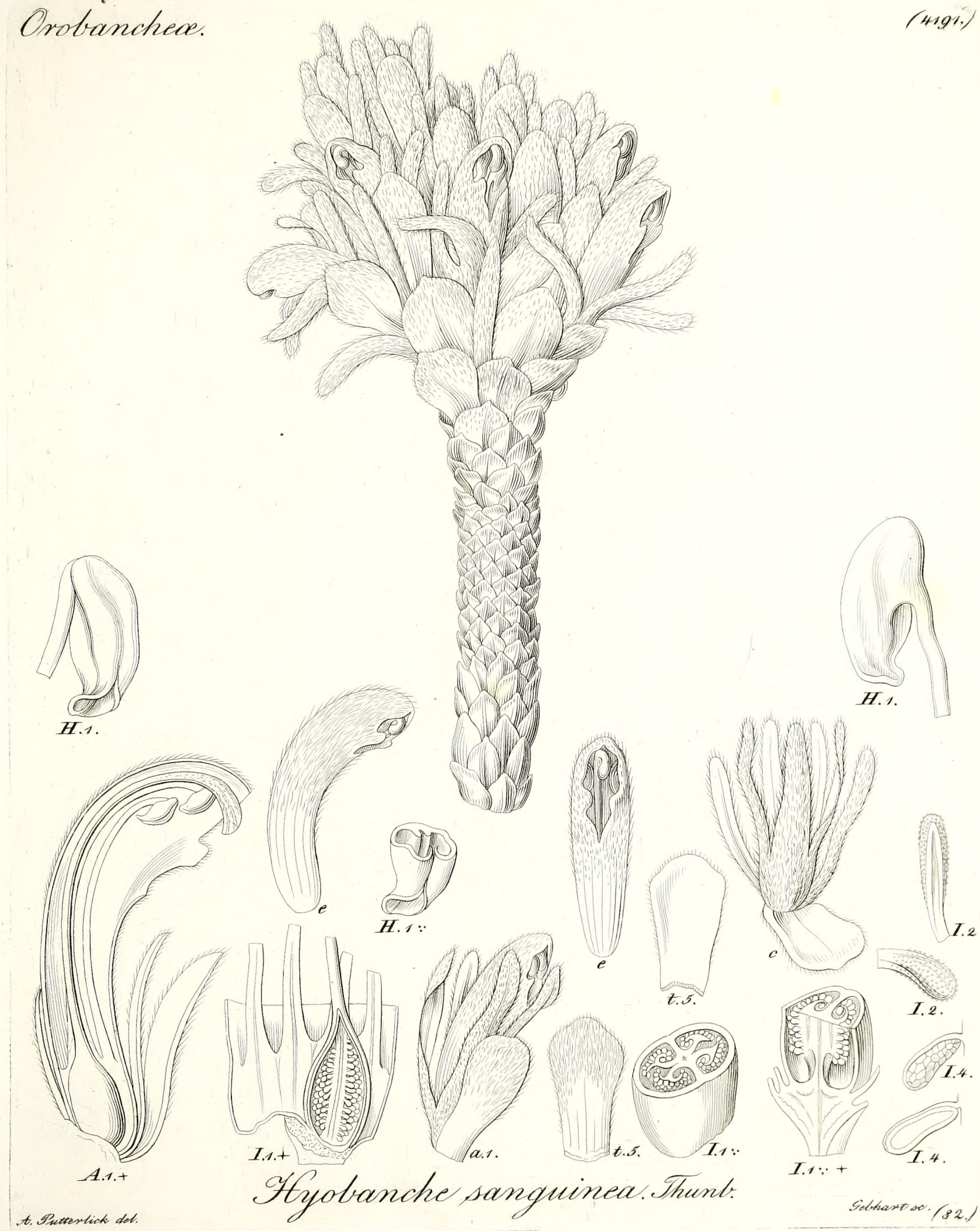

Sebhaxt oo (s2) 
Sapotacese.

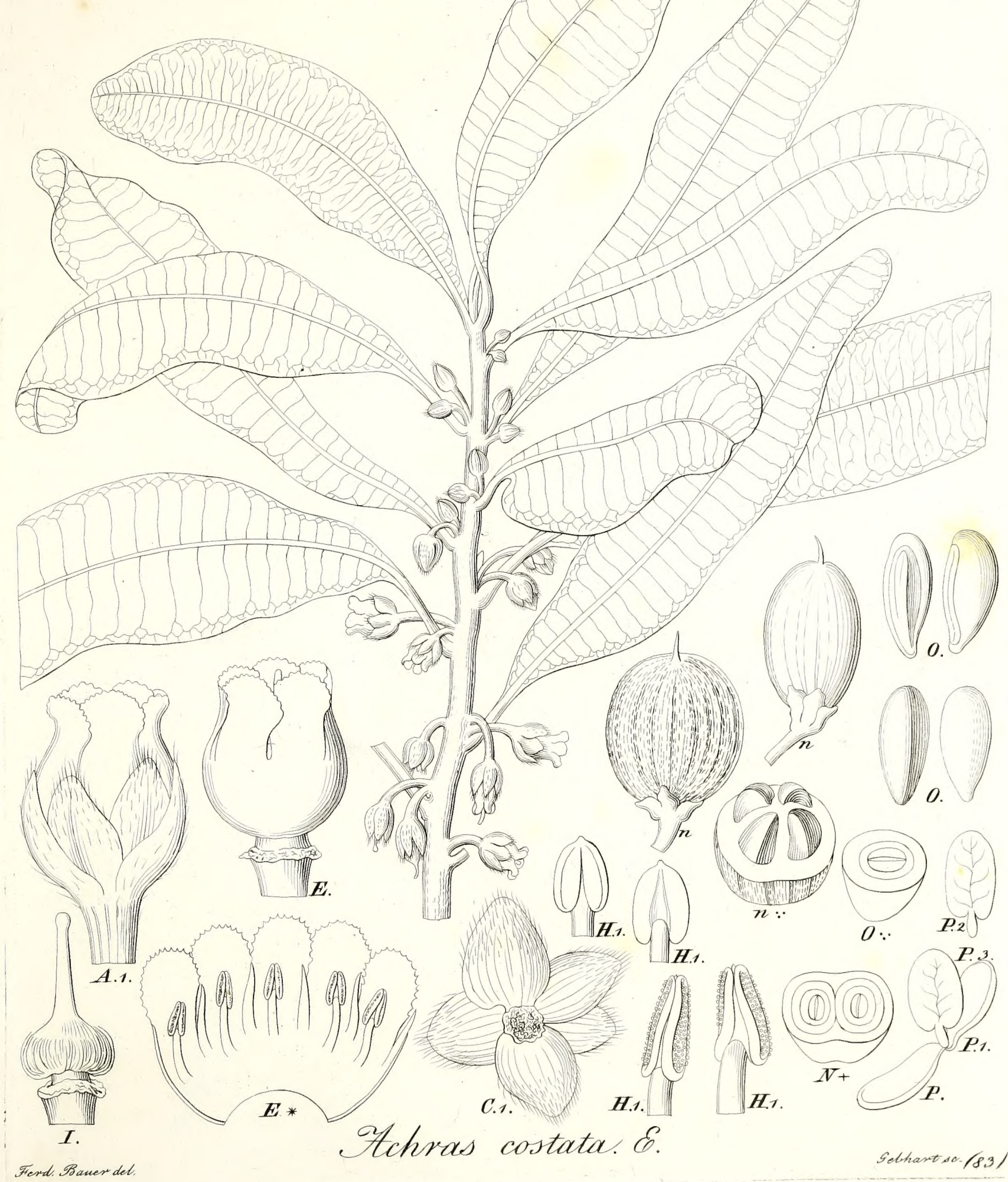


Gotumelliacea.

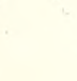

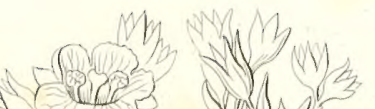

(4264.)

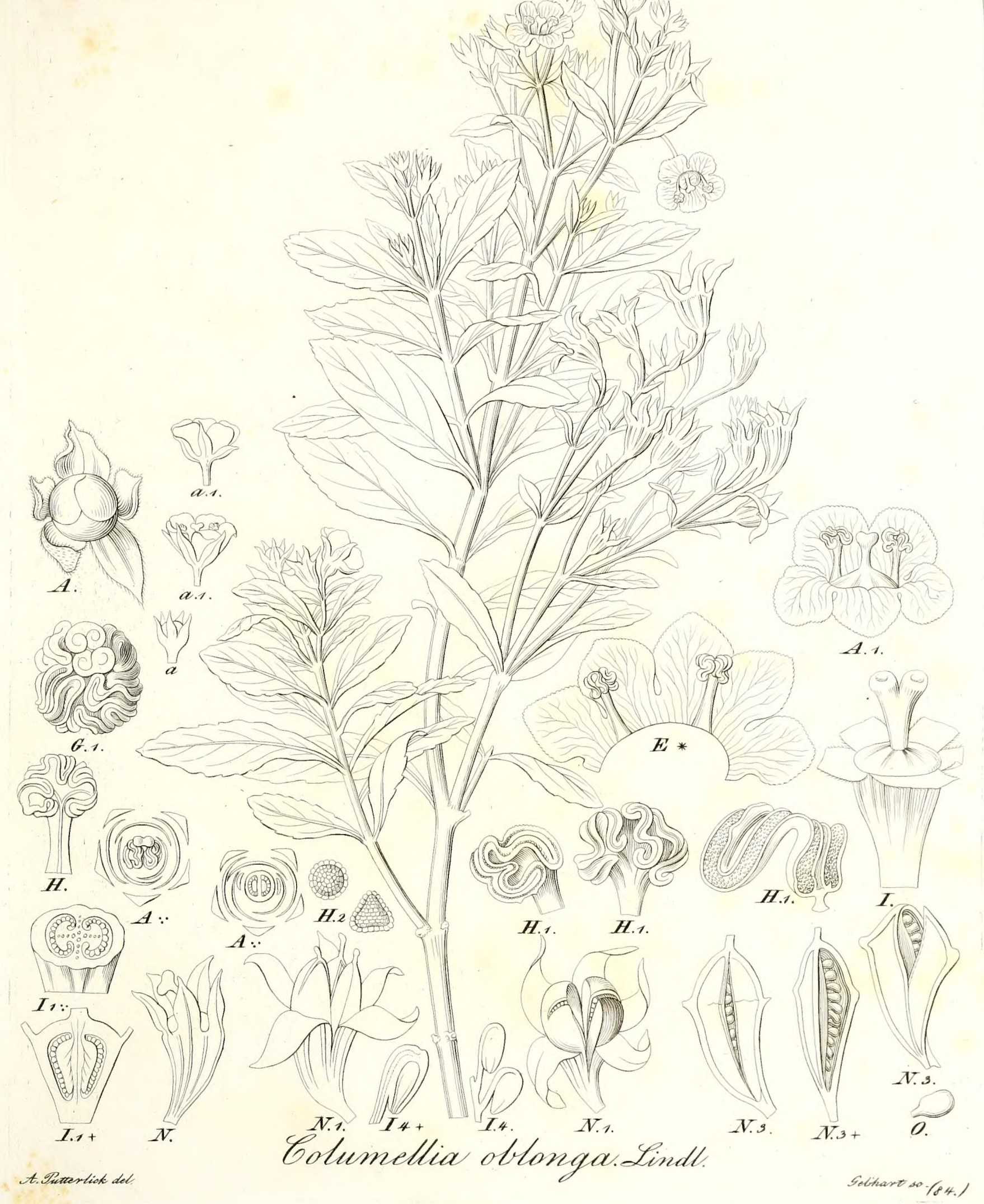


Rubiacece.
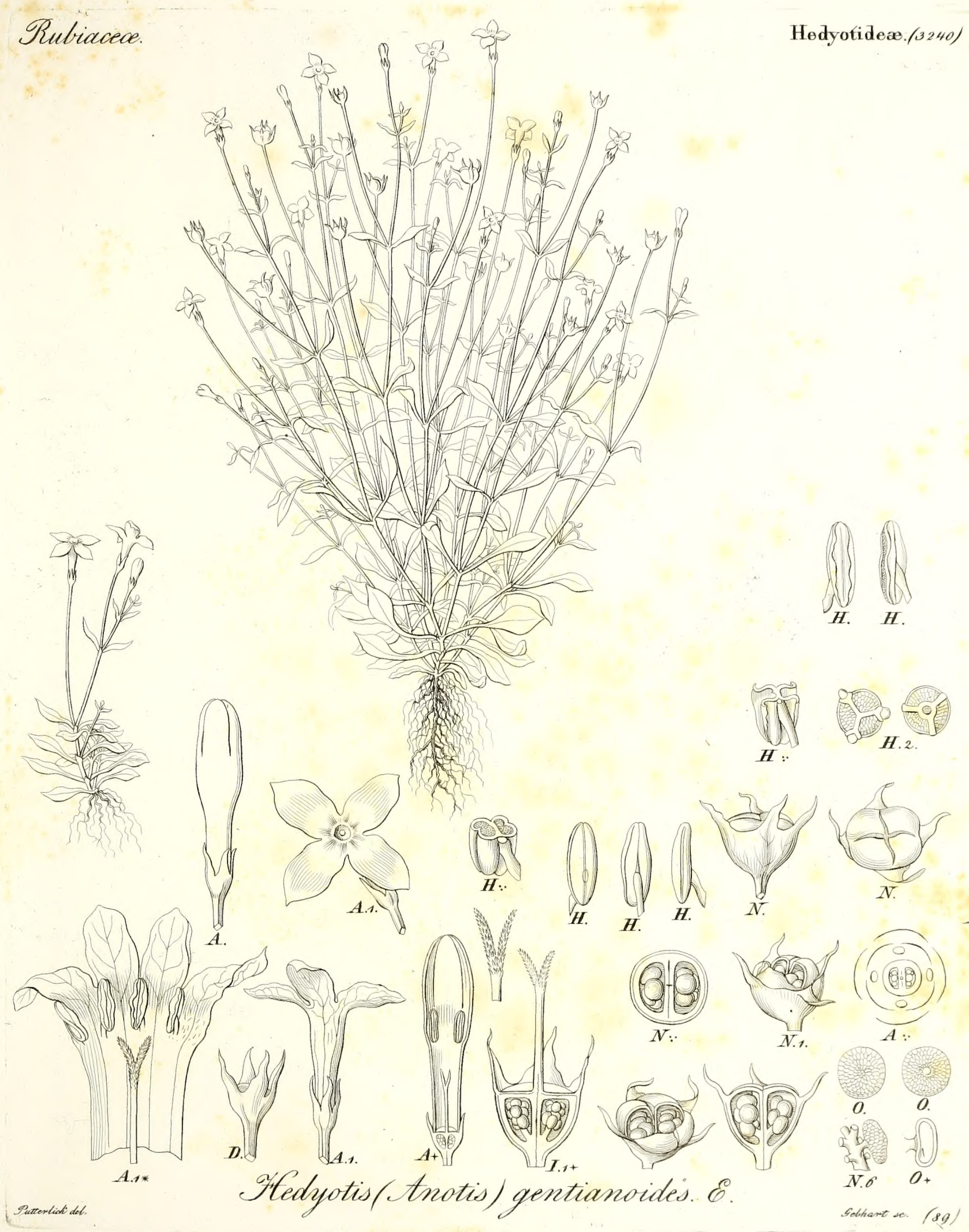

Hedyotidea.(3240) 


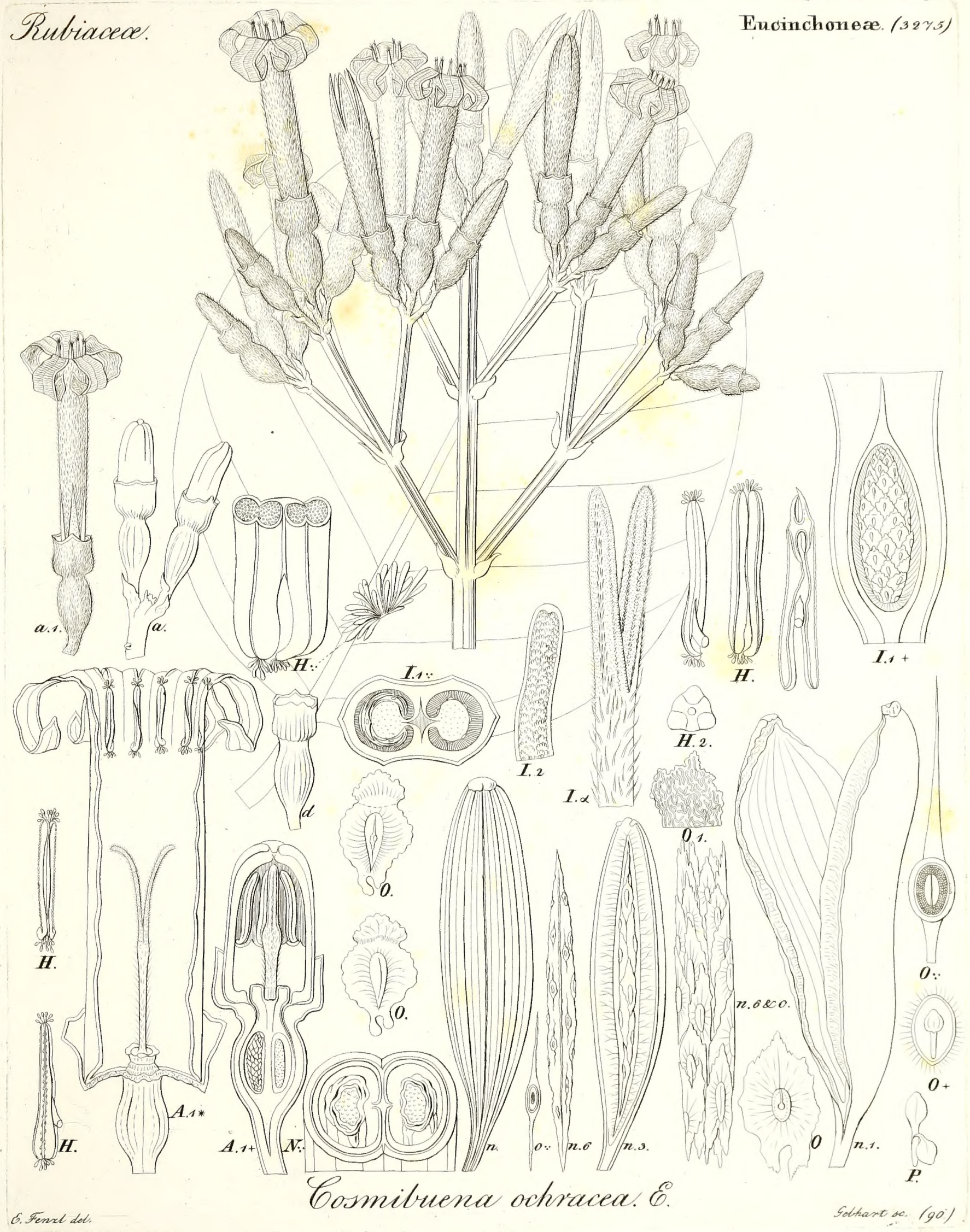




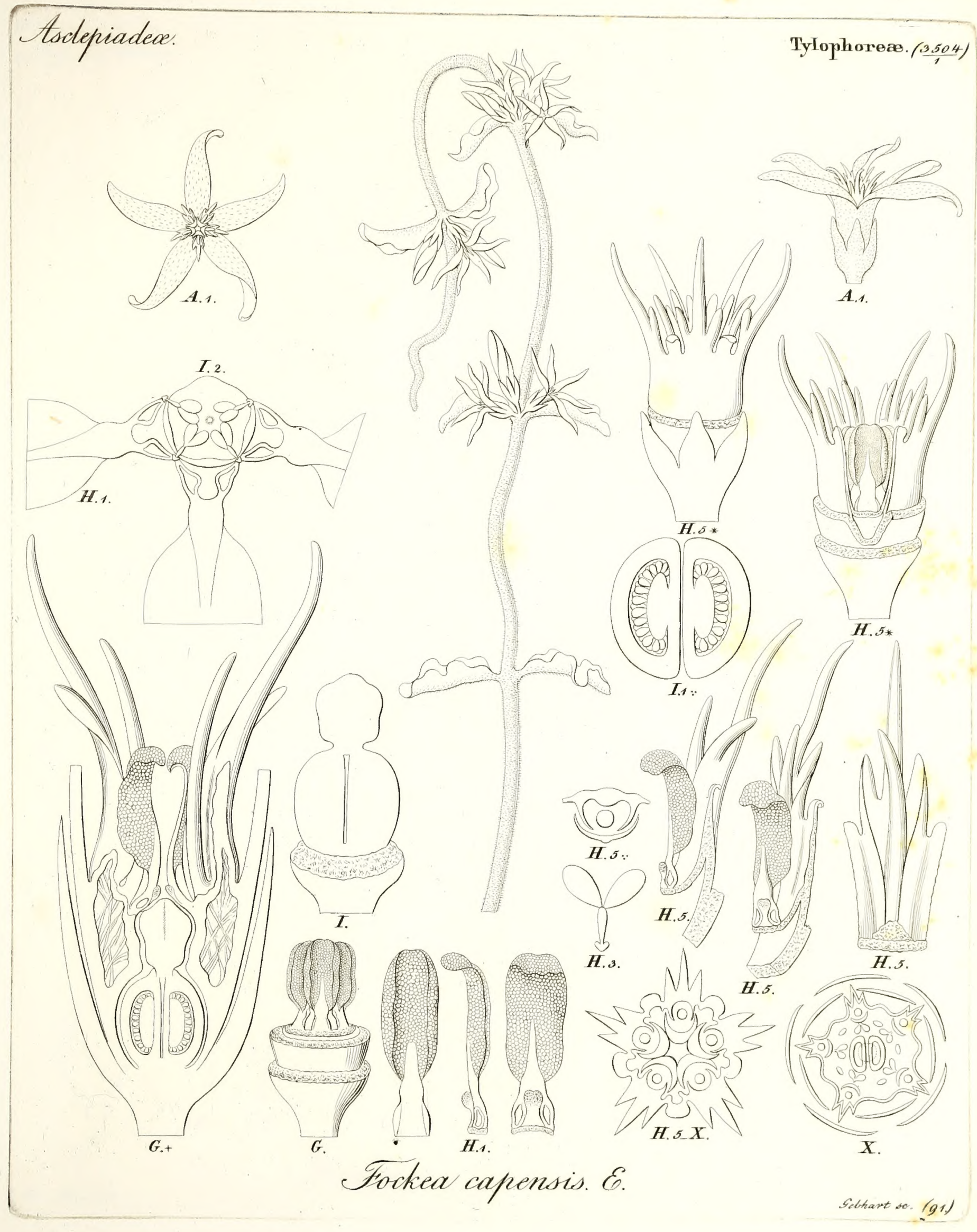


Myoporinede.
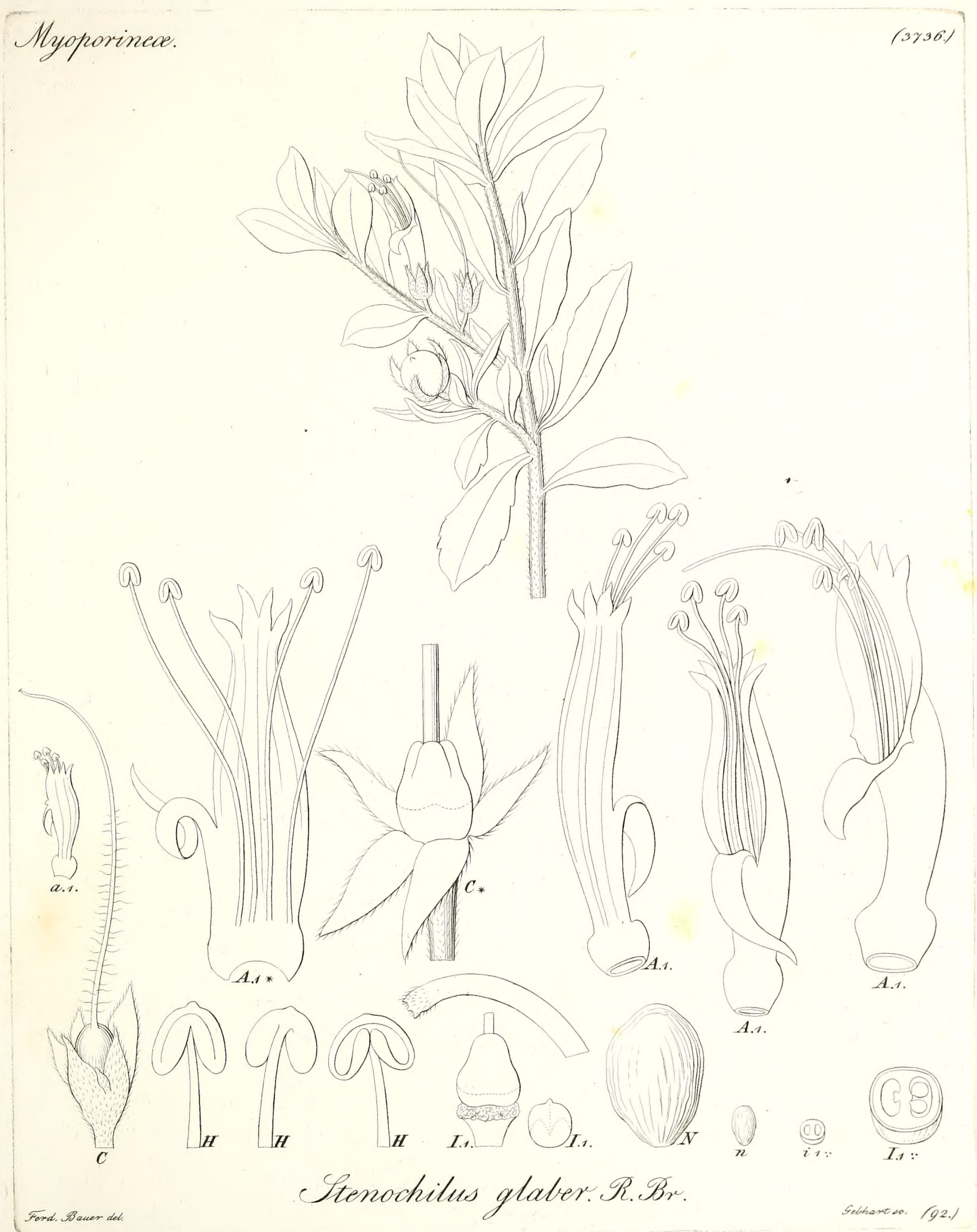

Lycopodiacex.
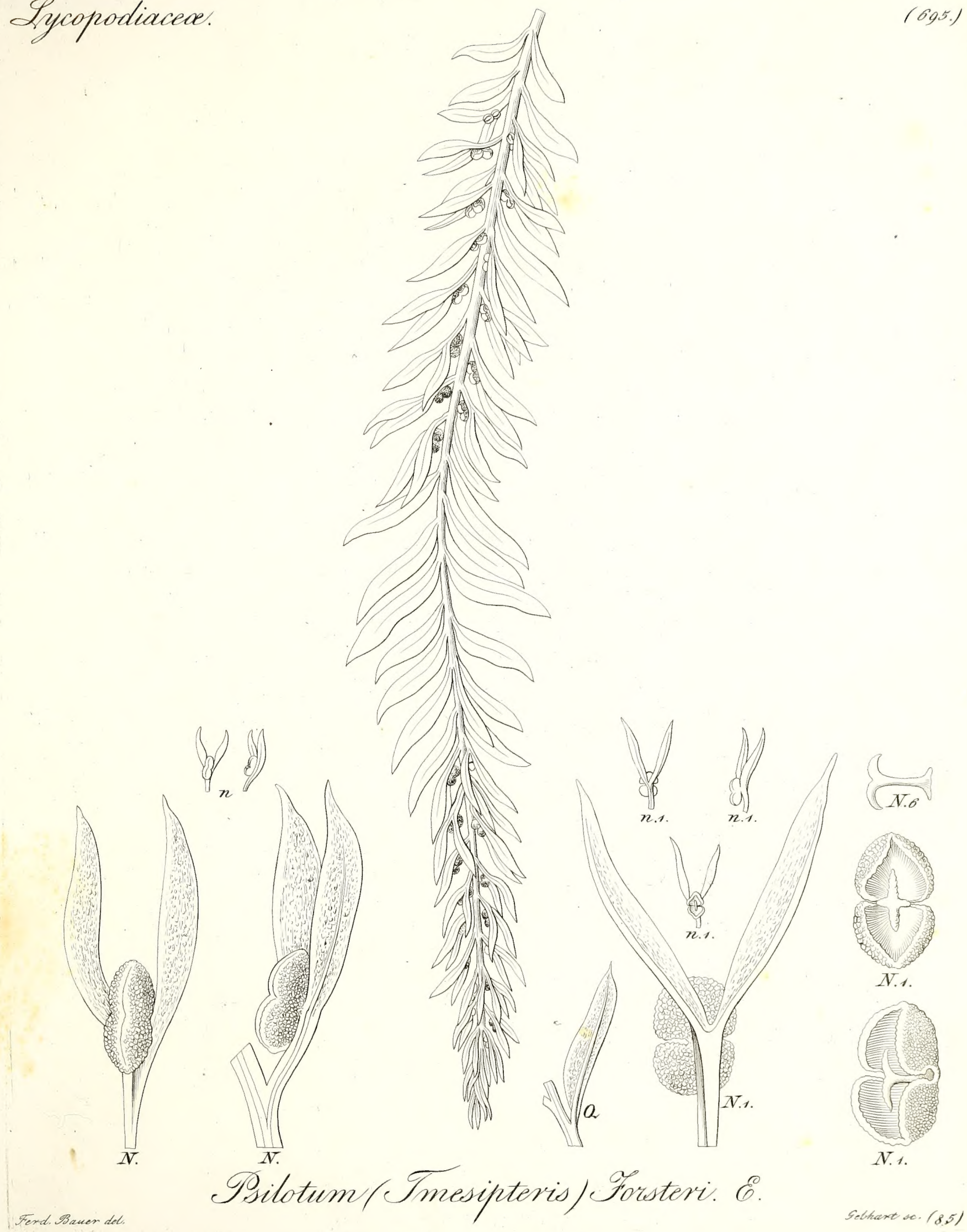

(6g5.) 
-

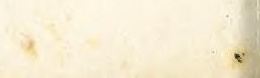




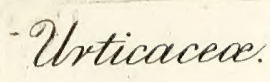

(1884)

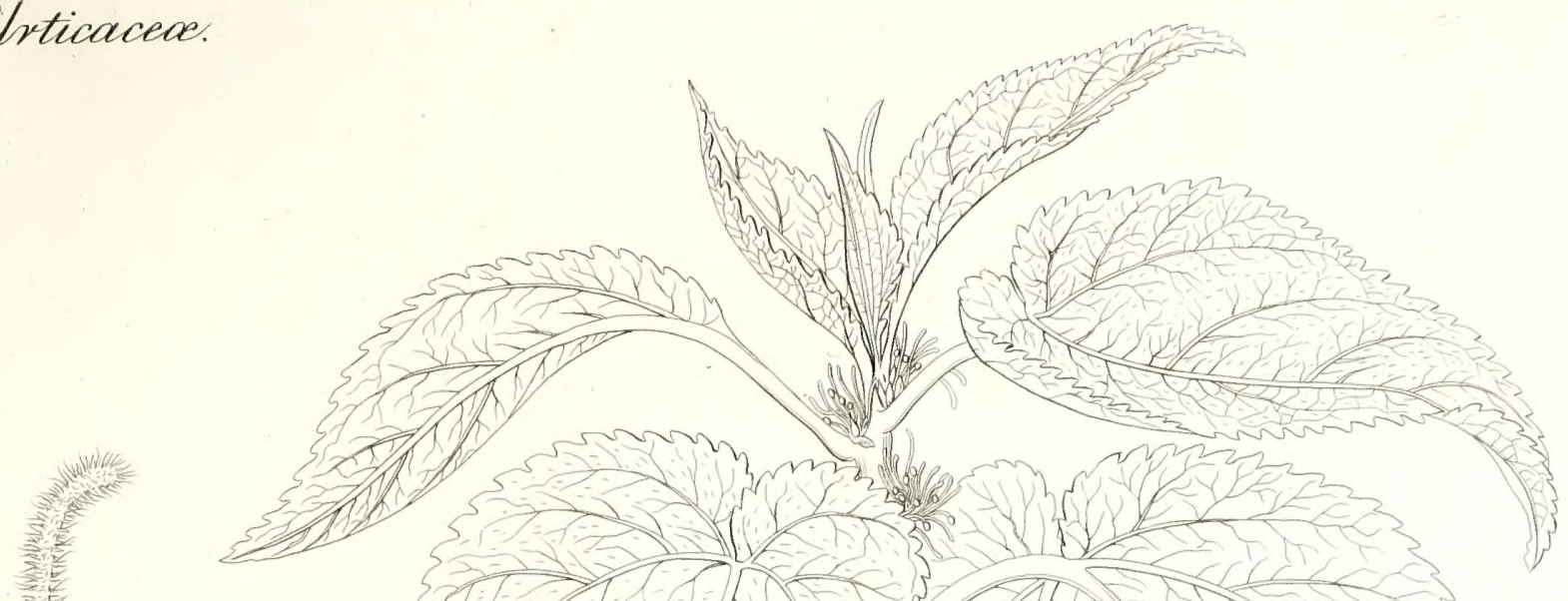





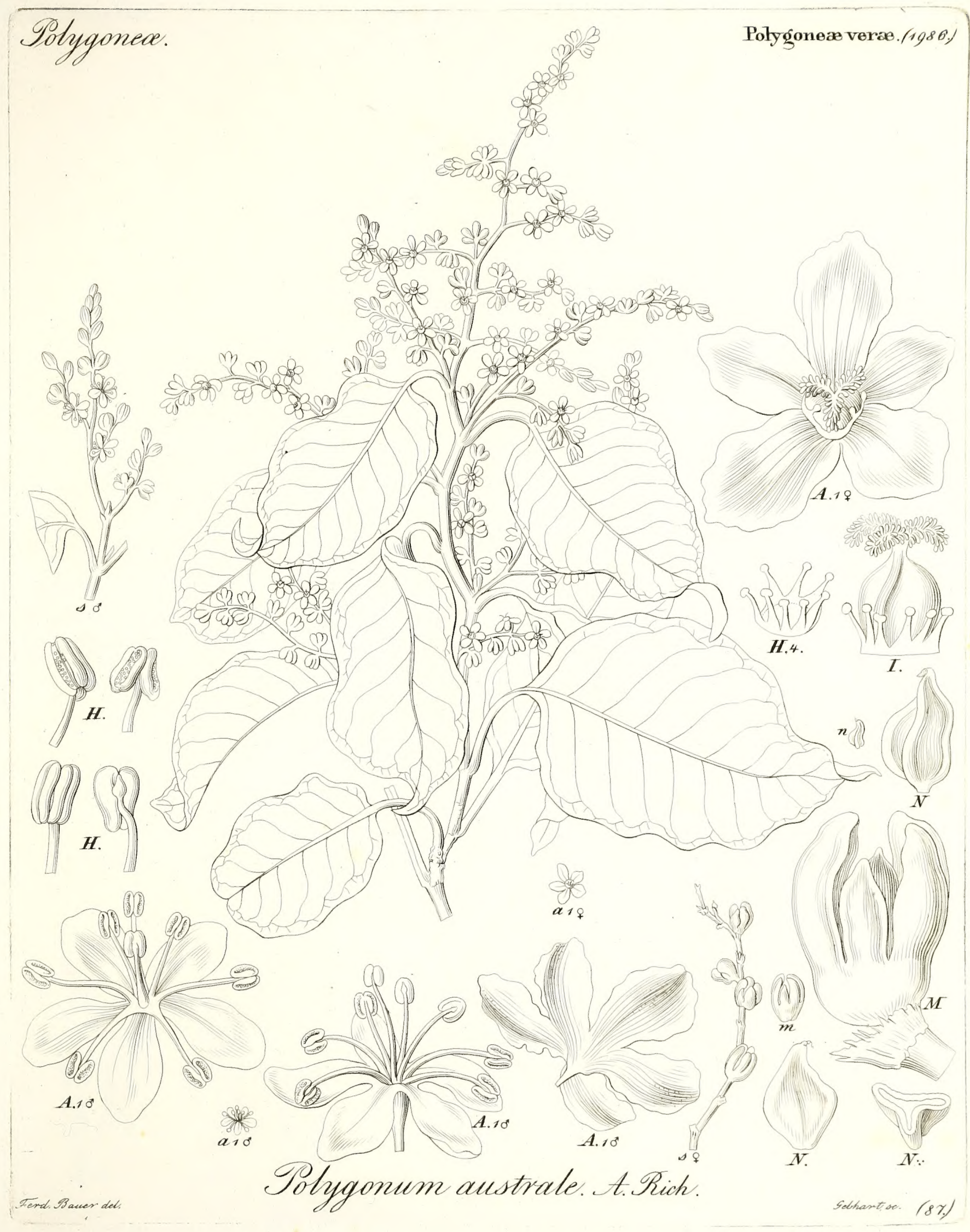


, 
Senecionidea.

Heliantheae. (2502)

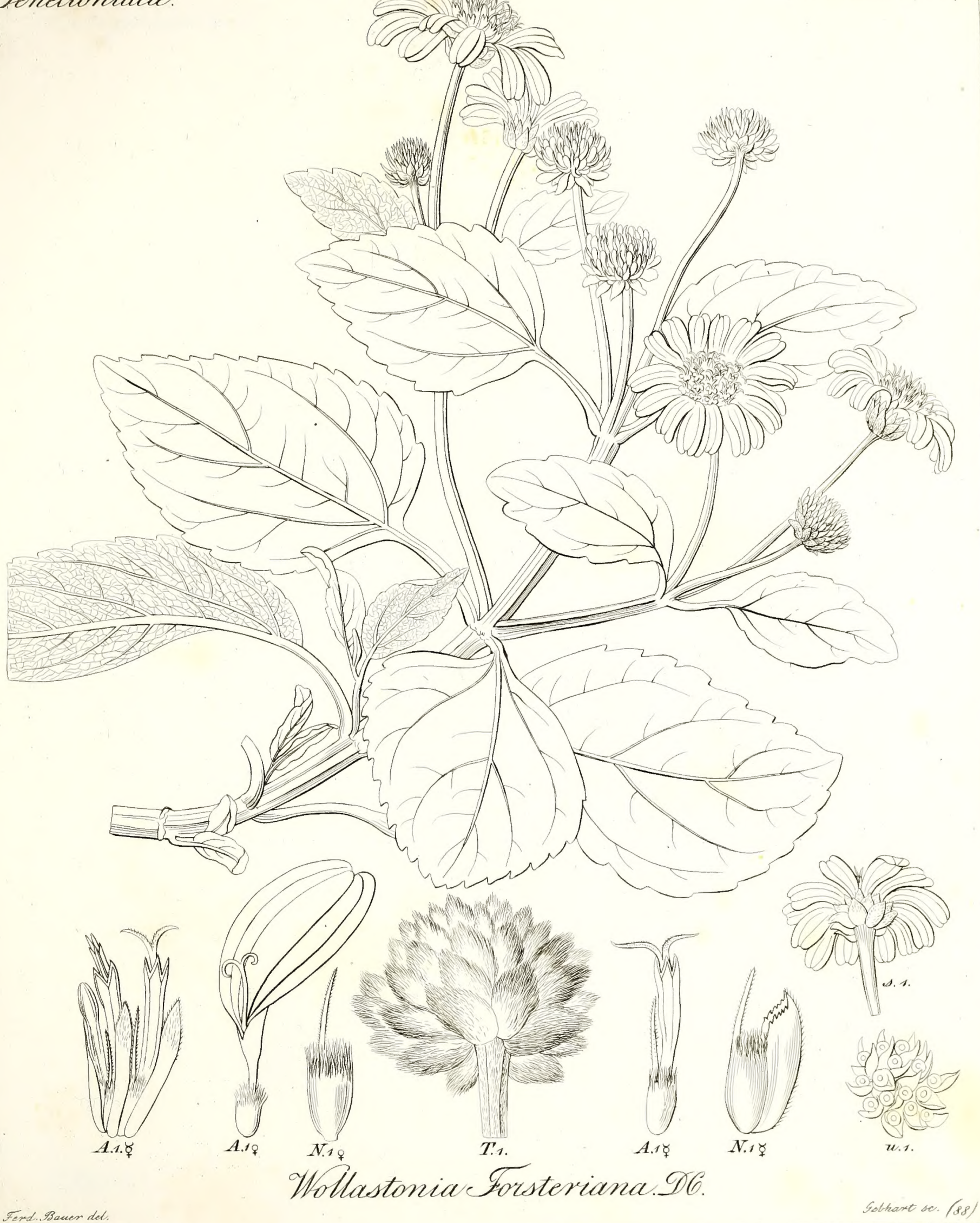


$x^{2}+y^{2}$ 
Serophularino.

Gratioleae.(3944)

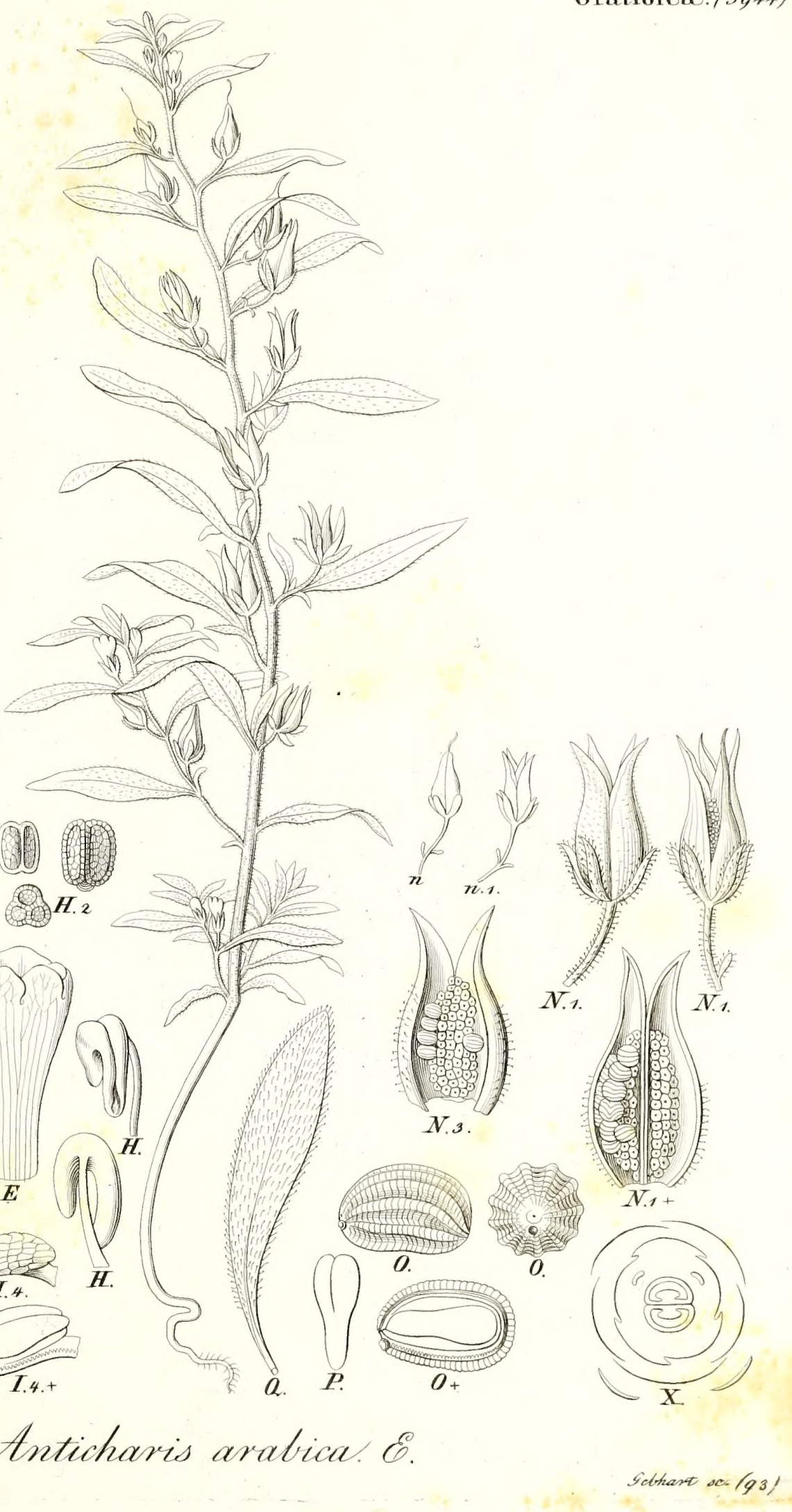




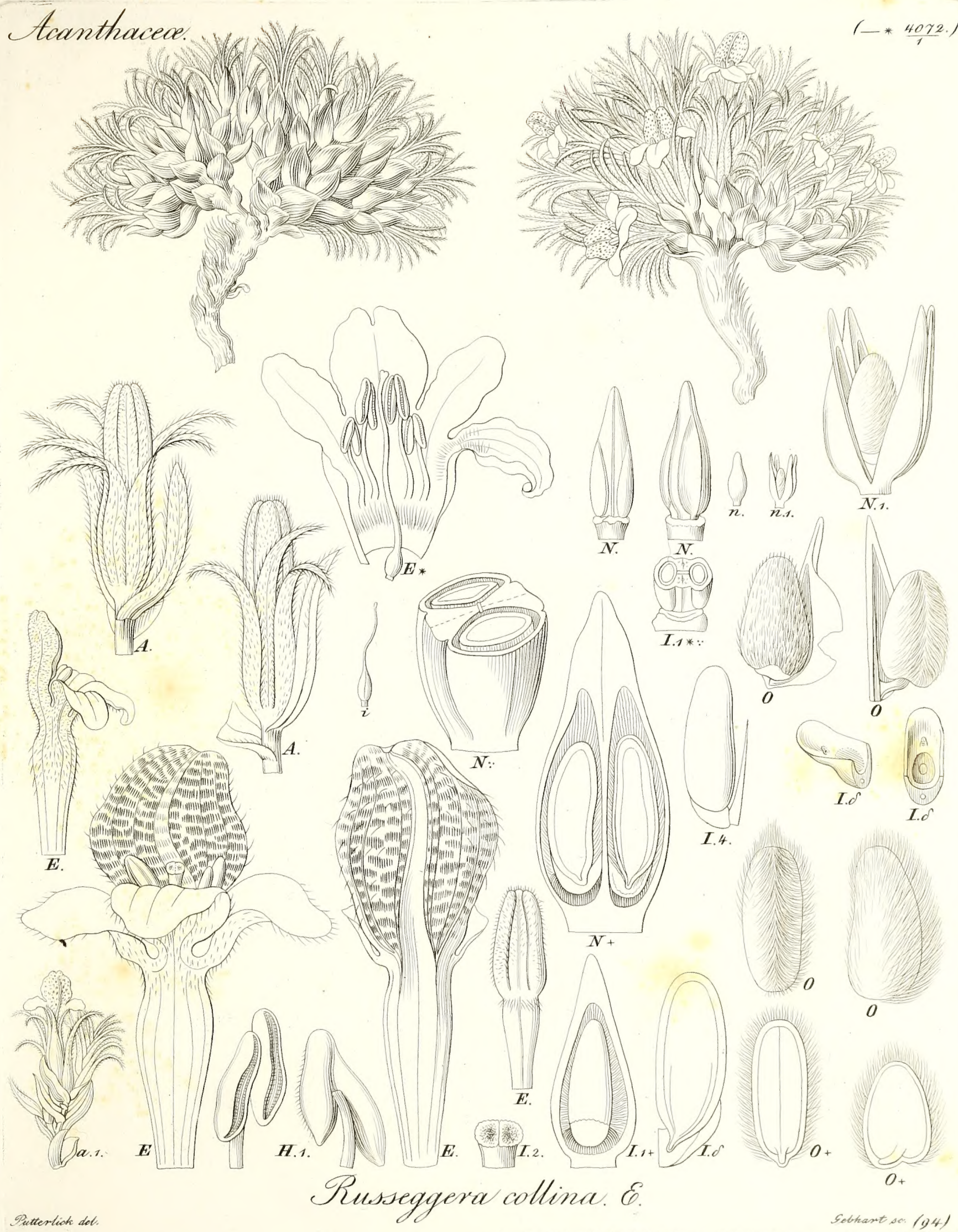




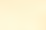

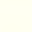



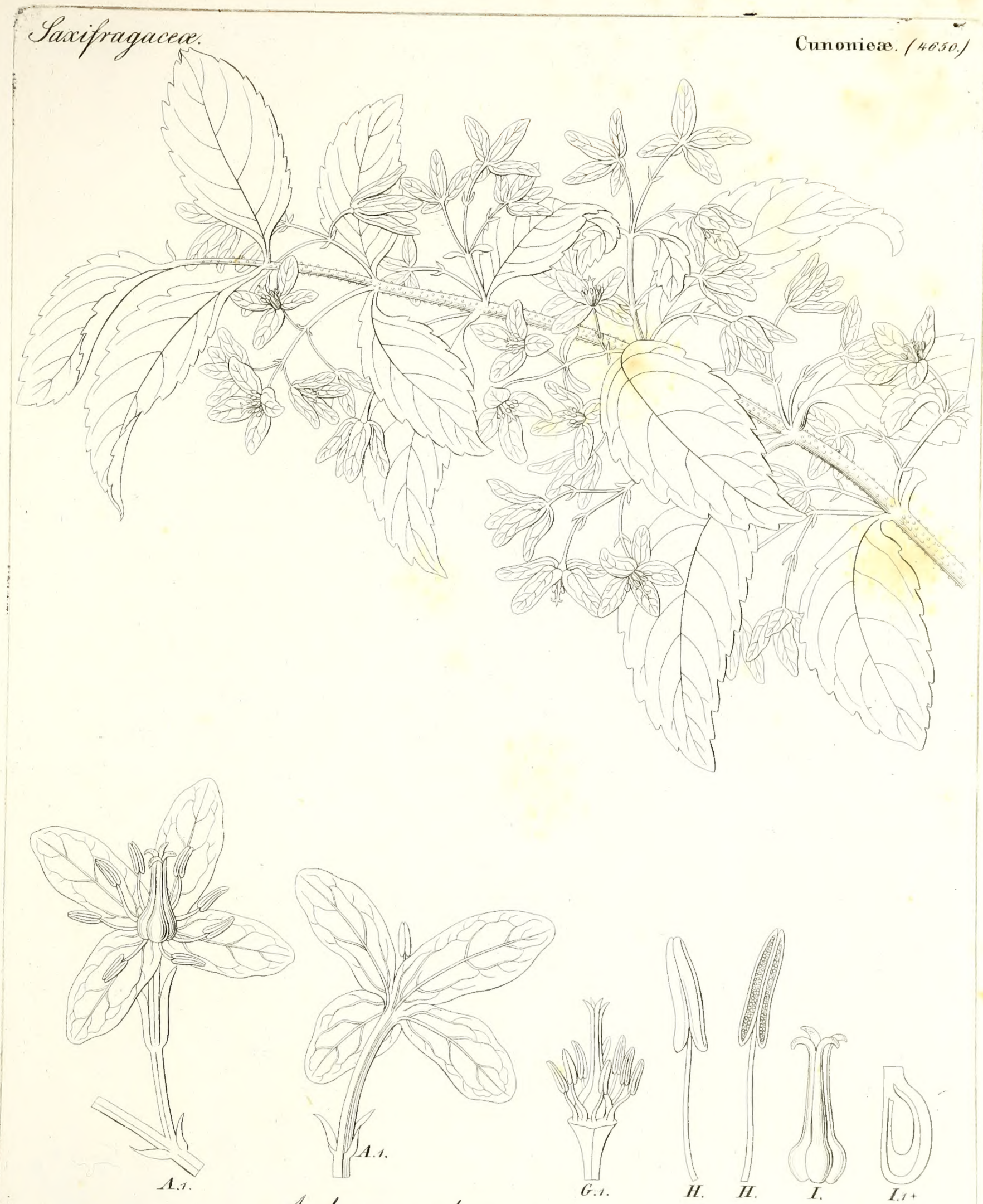

Aphanopetalum resinosum.

Gelhart se. (96.) 
$\because x^{2}+4 x^{2}$

|at

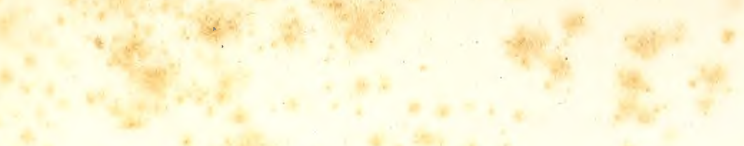


Siliaced.

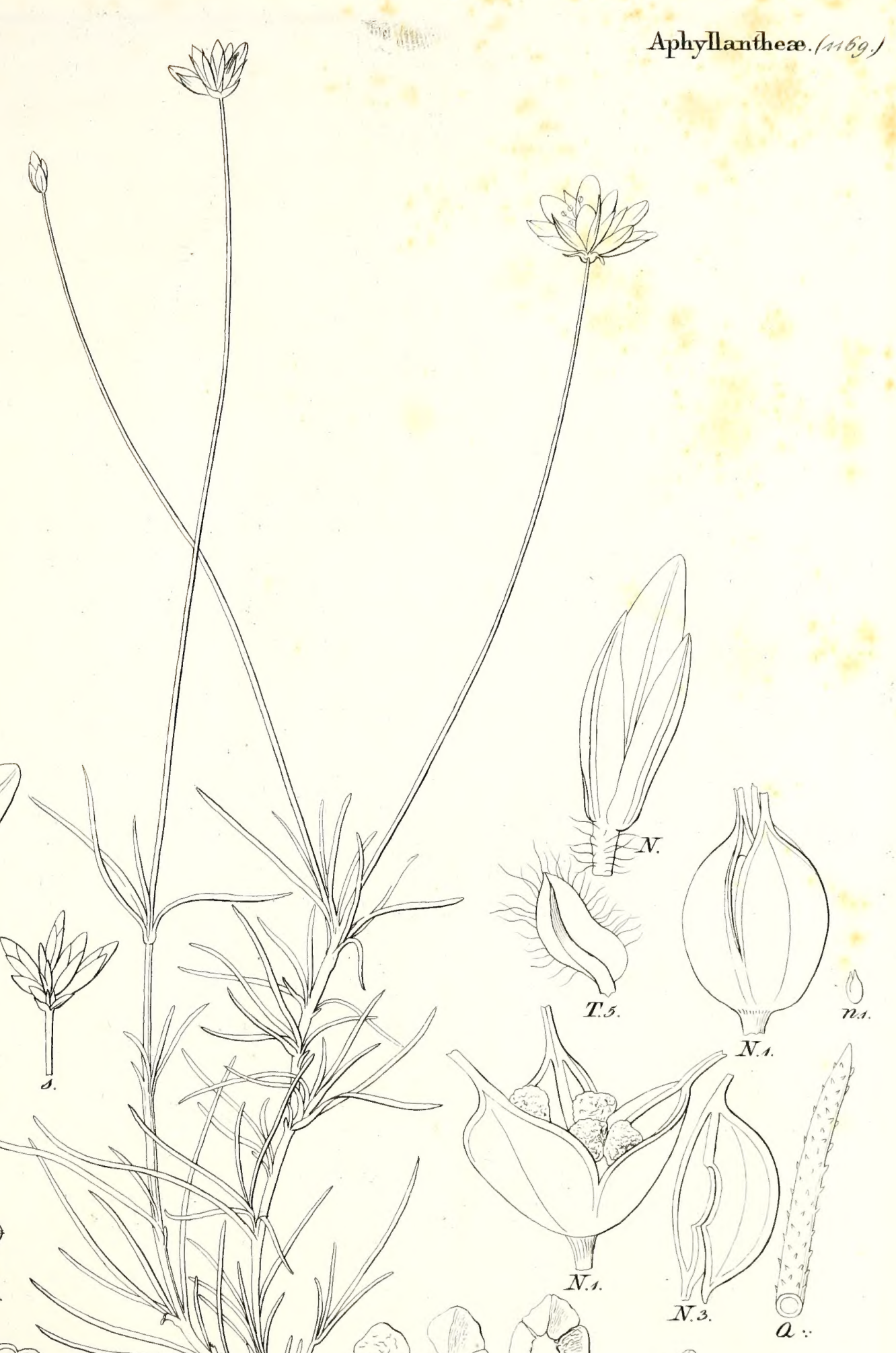
Ferd. Baneer del. Laxmannia qracilis. Re Rr. 



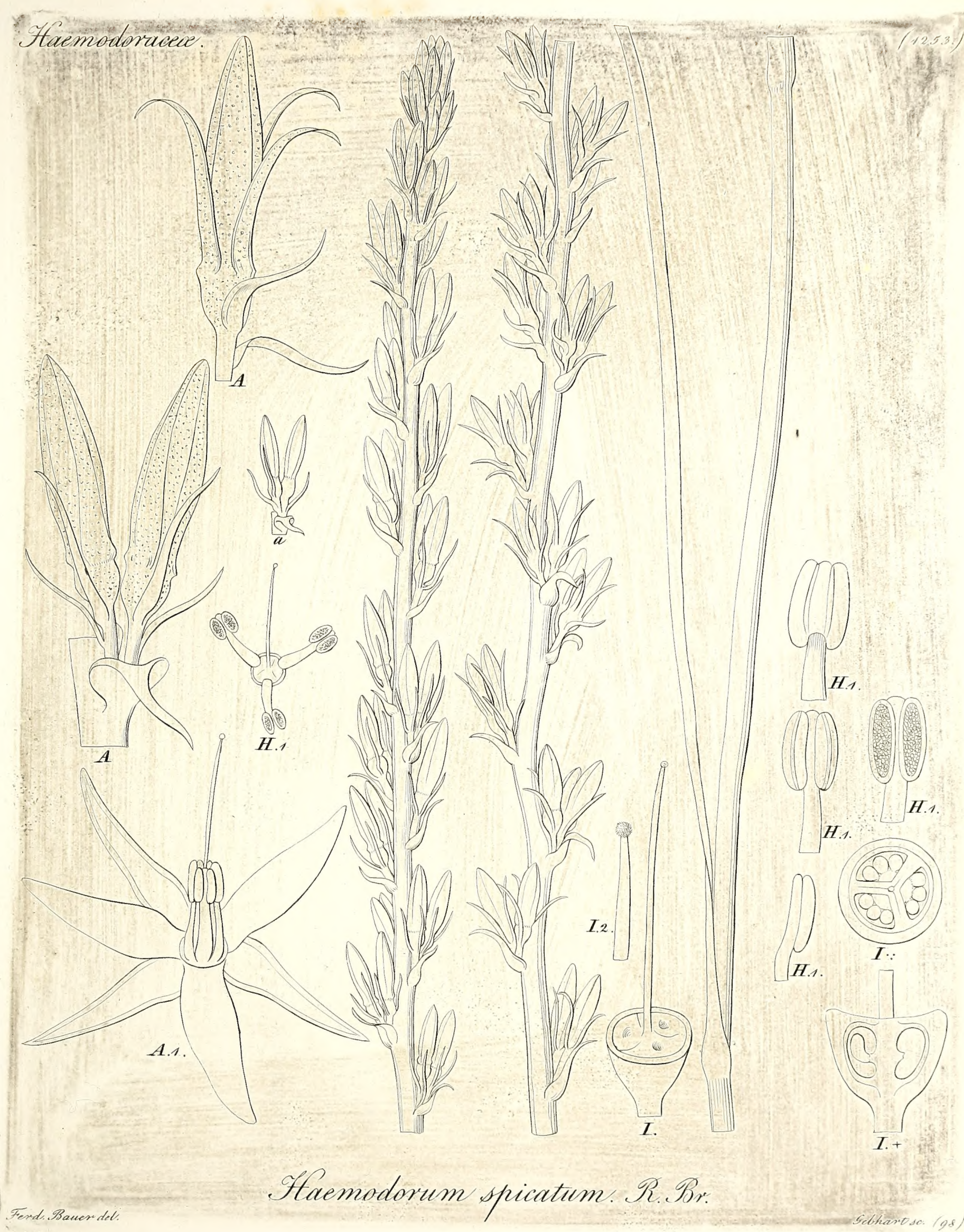




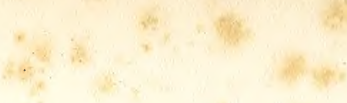

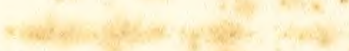


Orchidew.

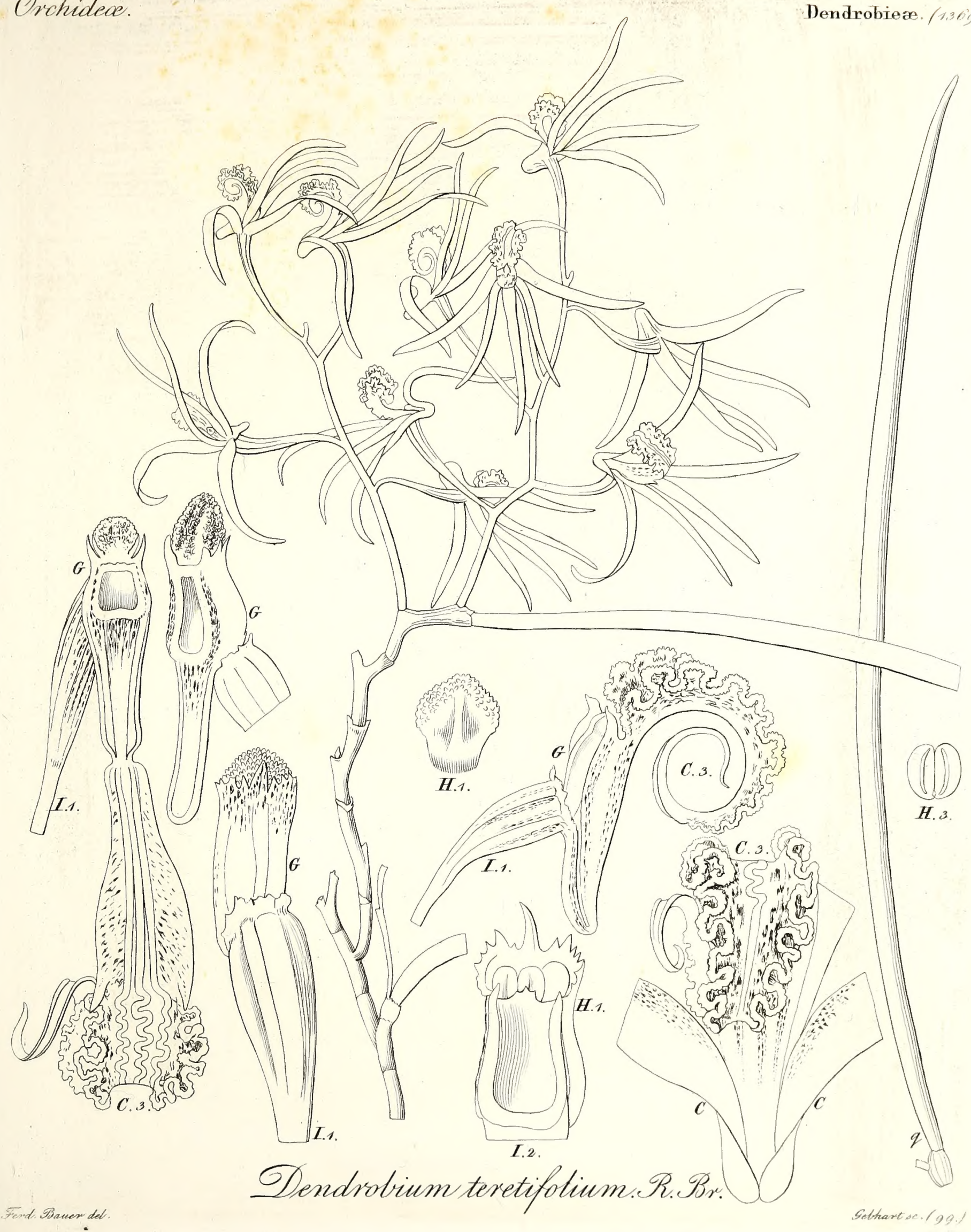


Ghenopodece.

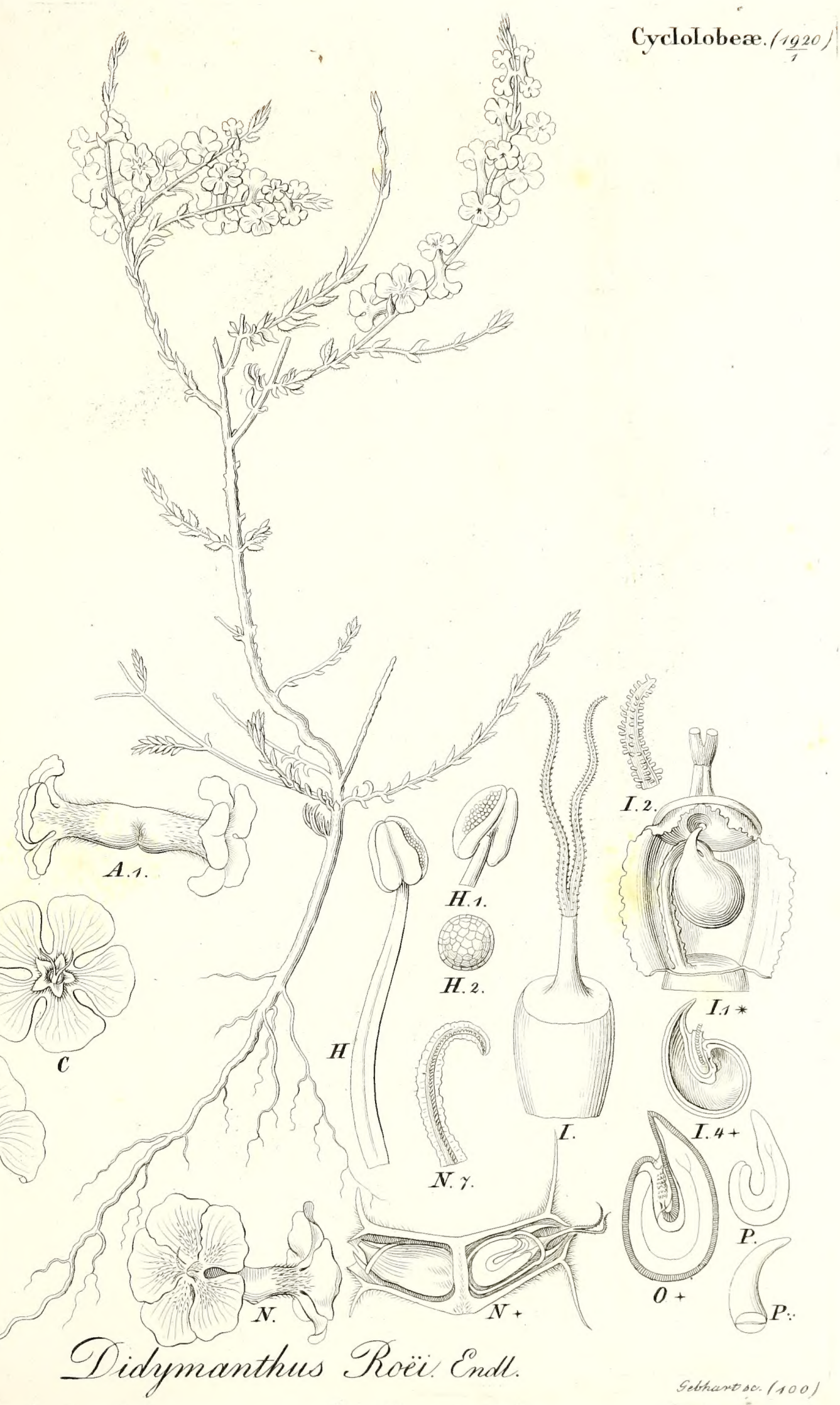




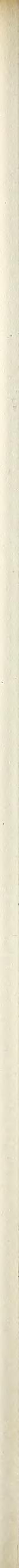


Loganiacexe.

Coelostyleae $/ \frac{3360}{2}$

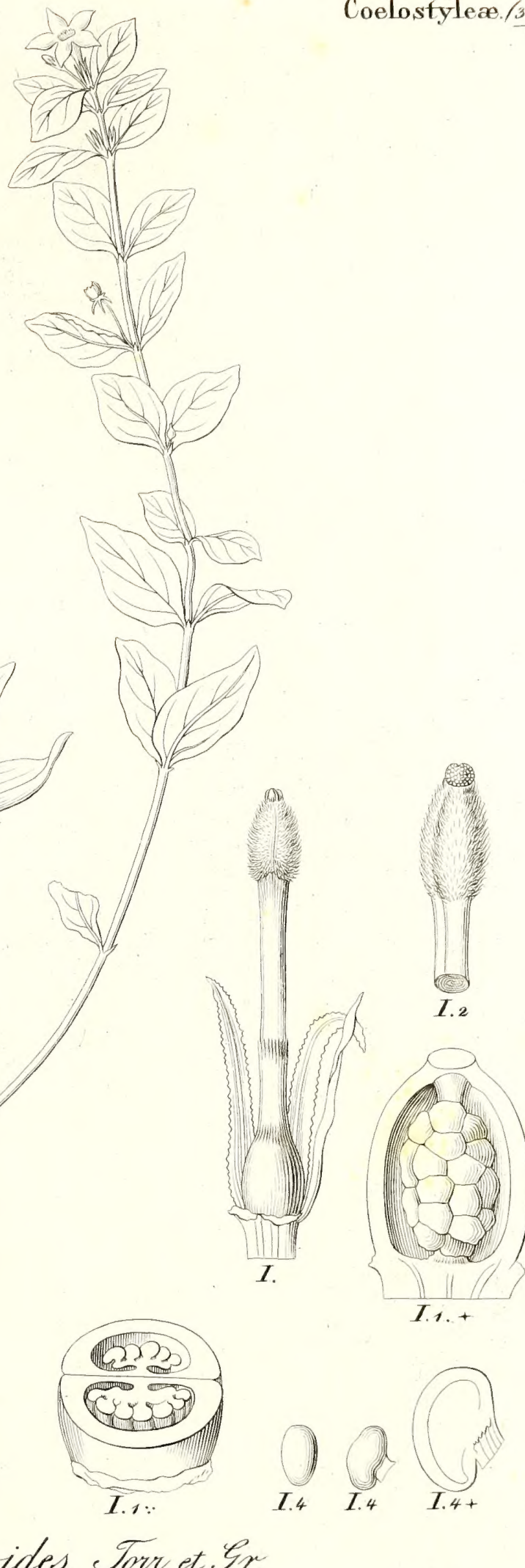

-1. Putterlich dod.

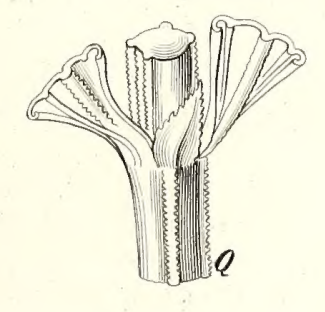



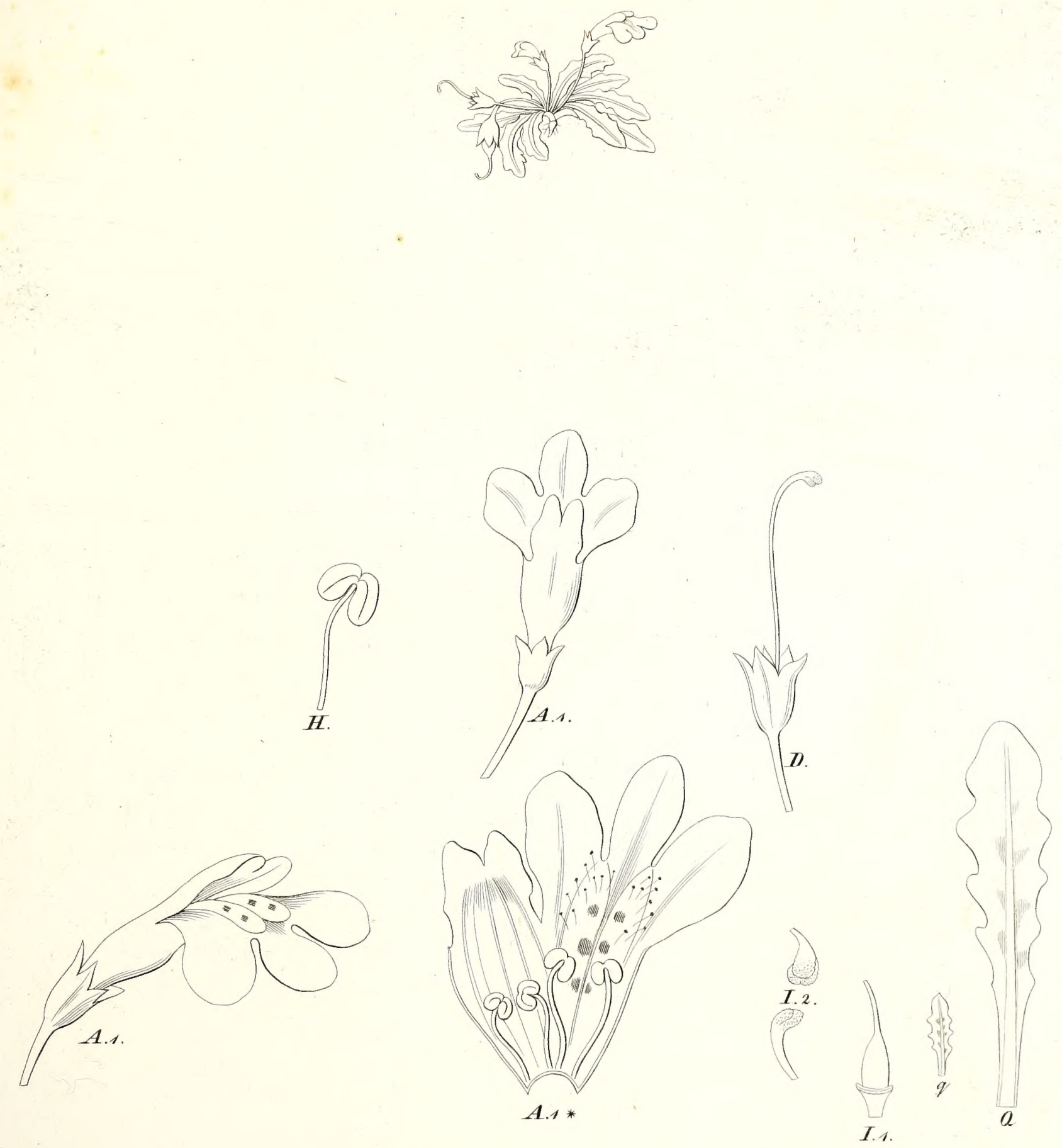

Marus Primilio. R. Rr. 
Scrophudarinoe.

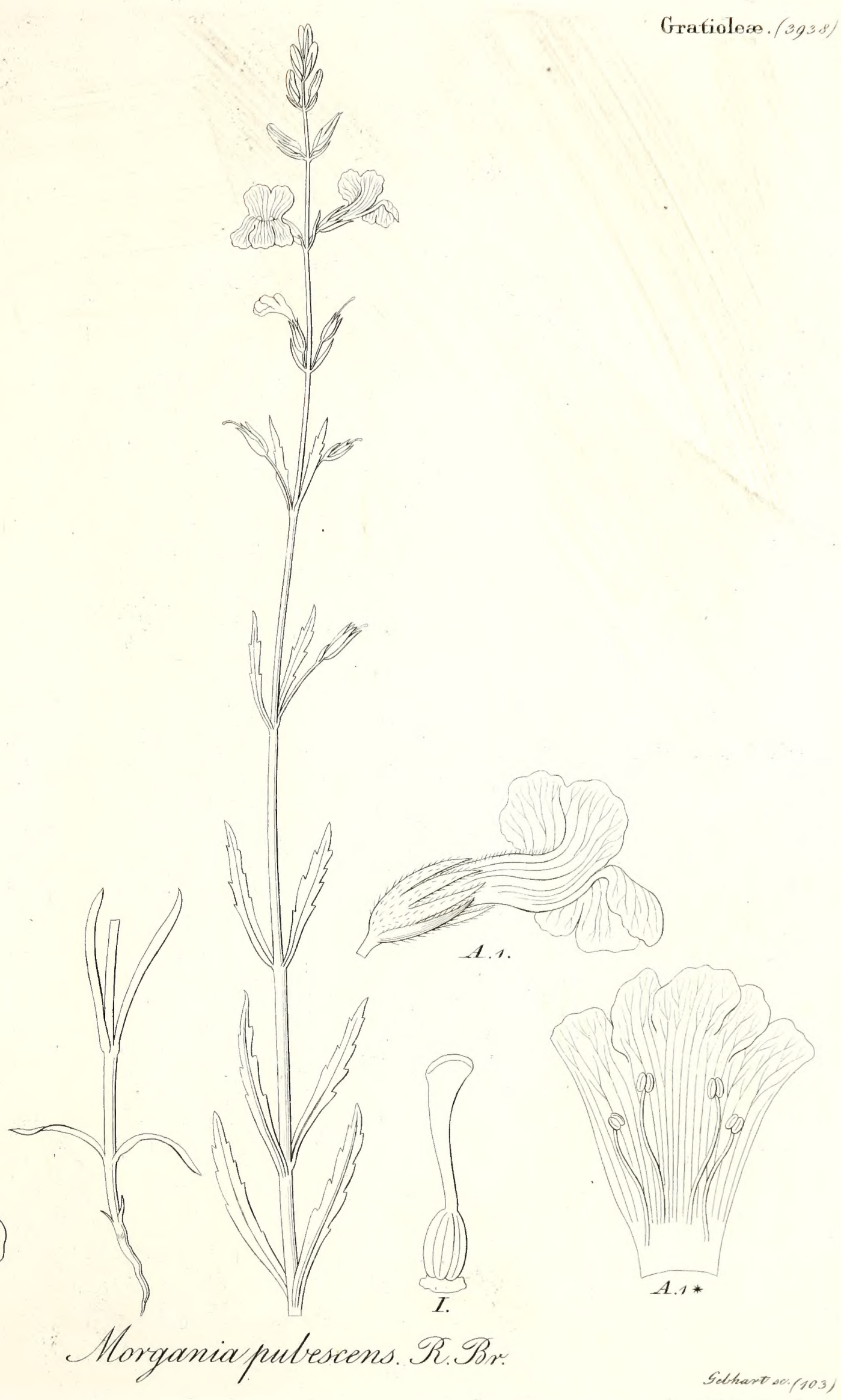



tranthacese.

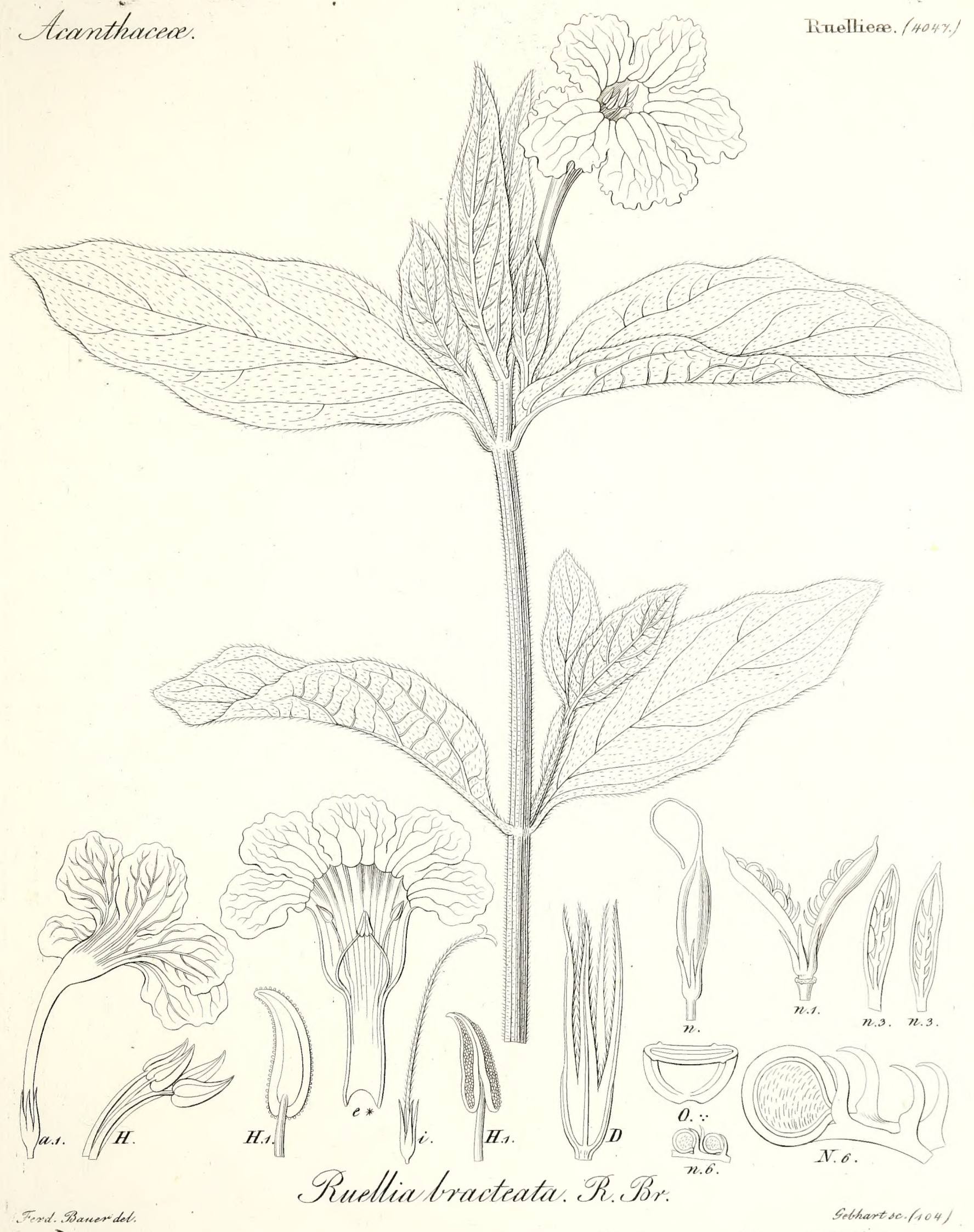

Ruellieae. (404\%) 
tianthacear.

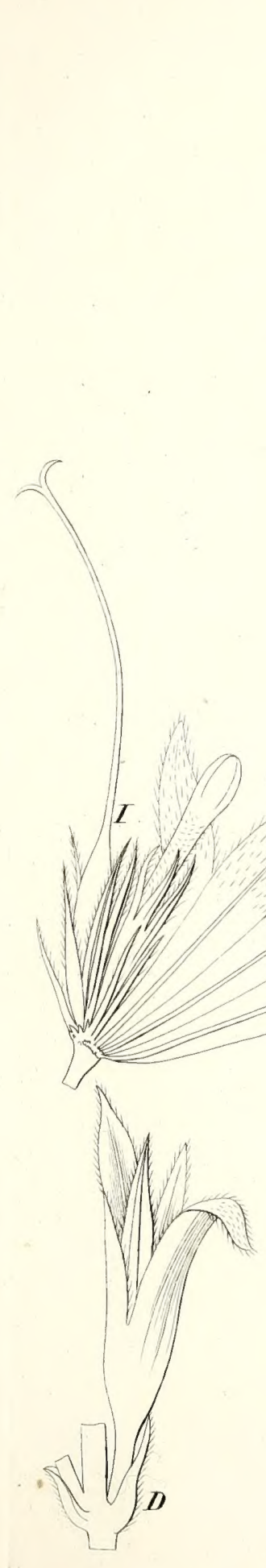

Ferd Bavere del

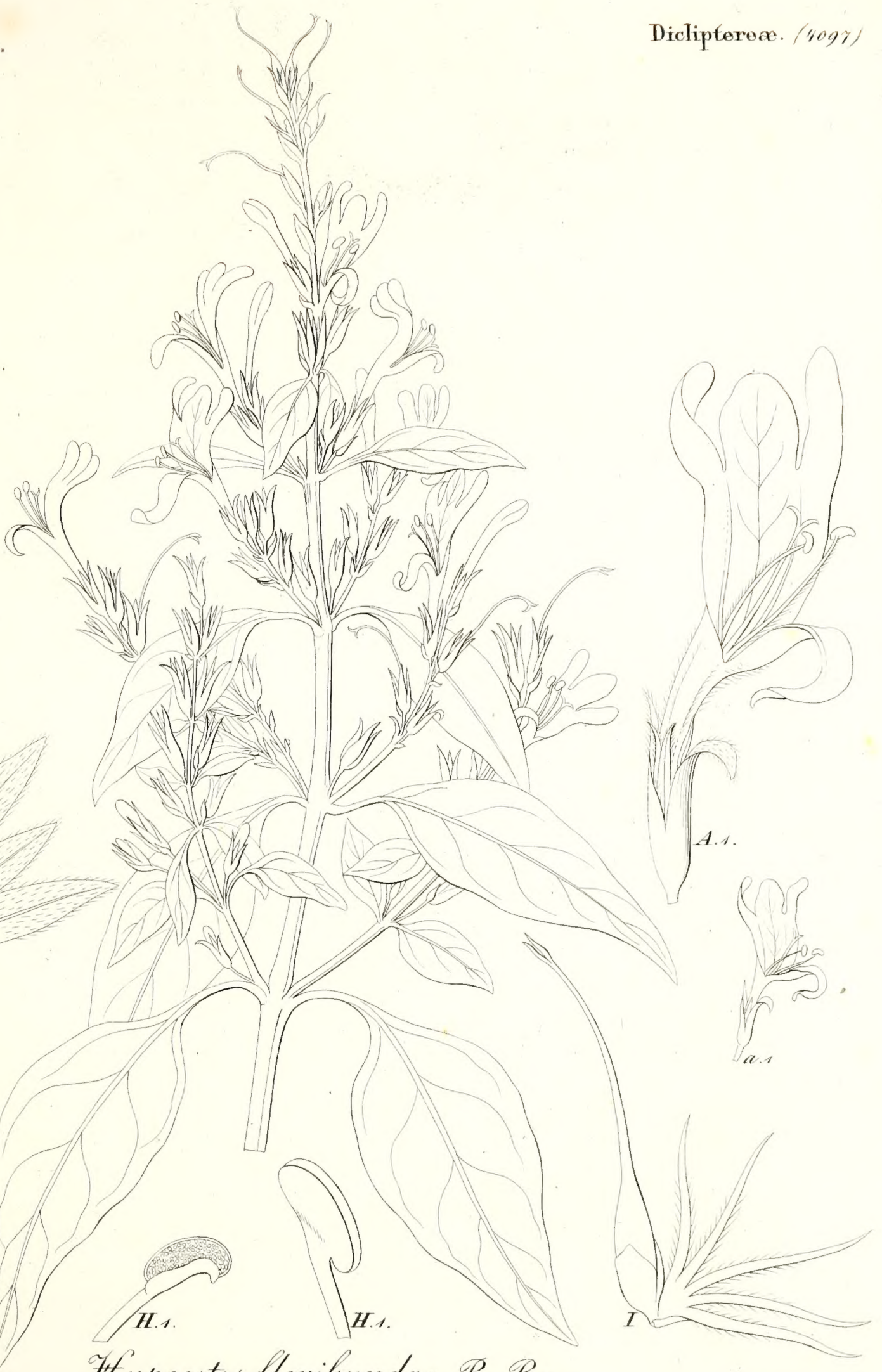

Itypoestes floribunda. R.B. 
Pedalinea.

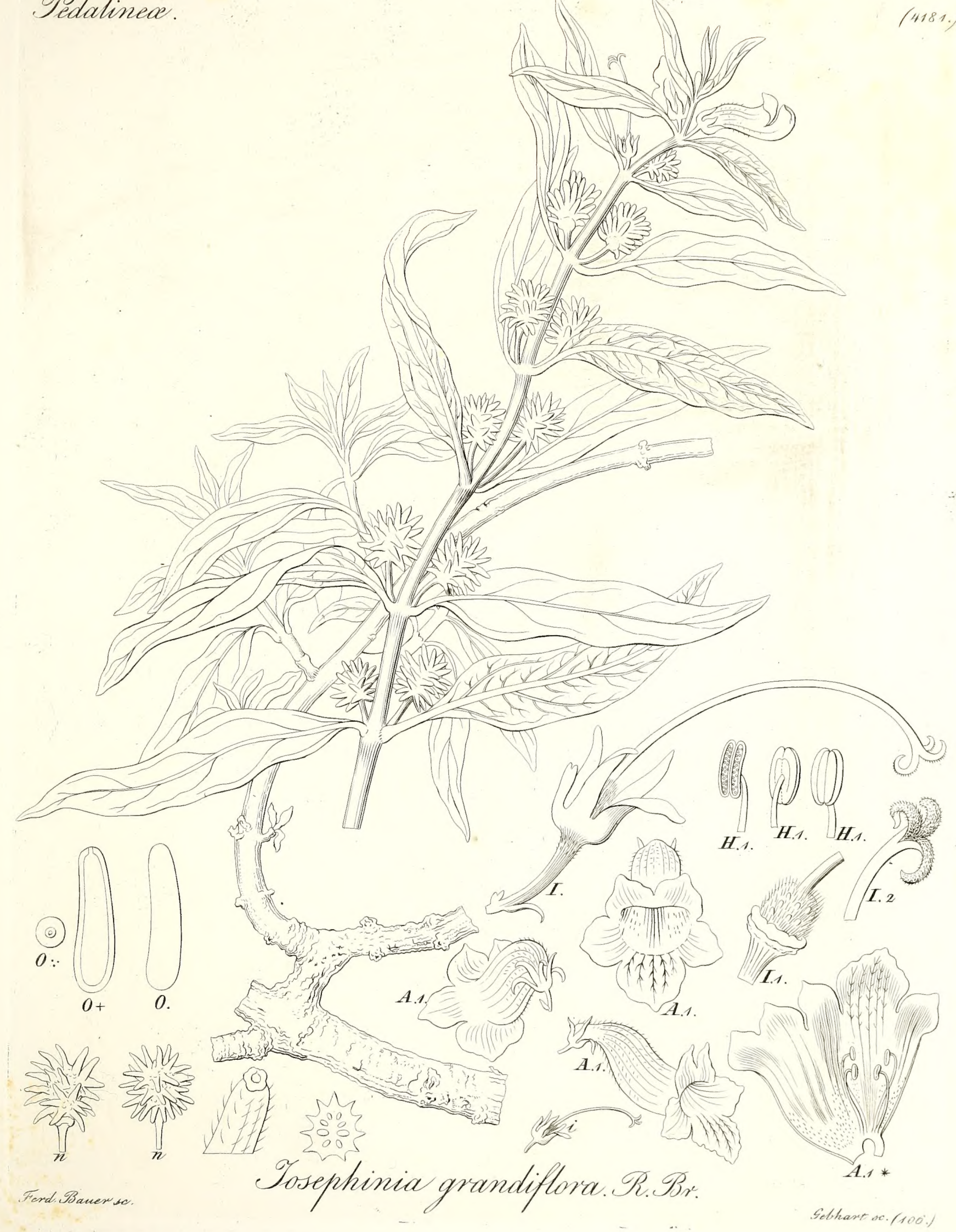




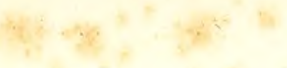

- 20

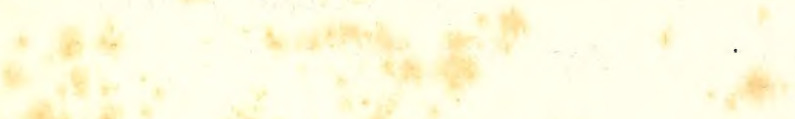

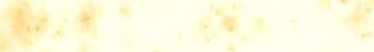




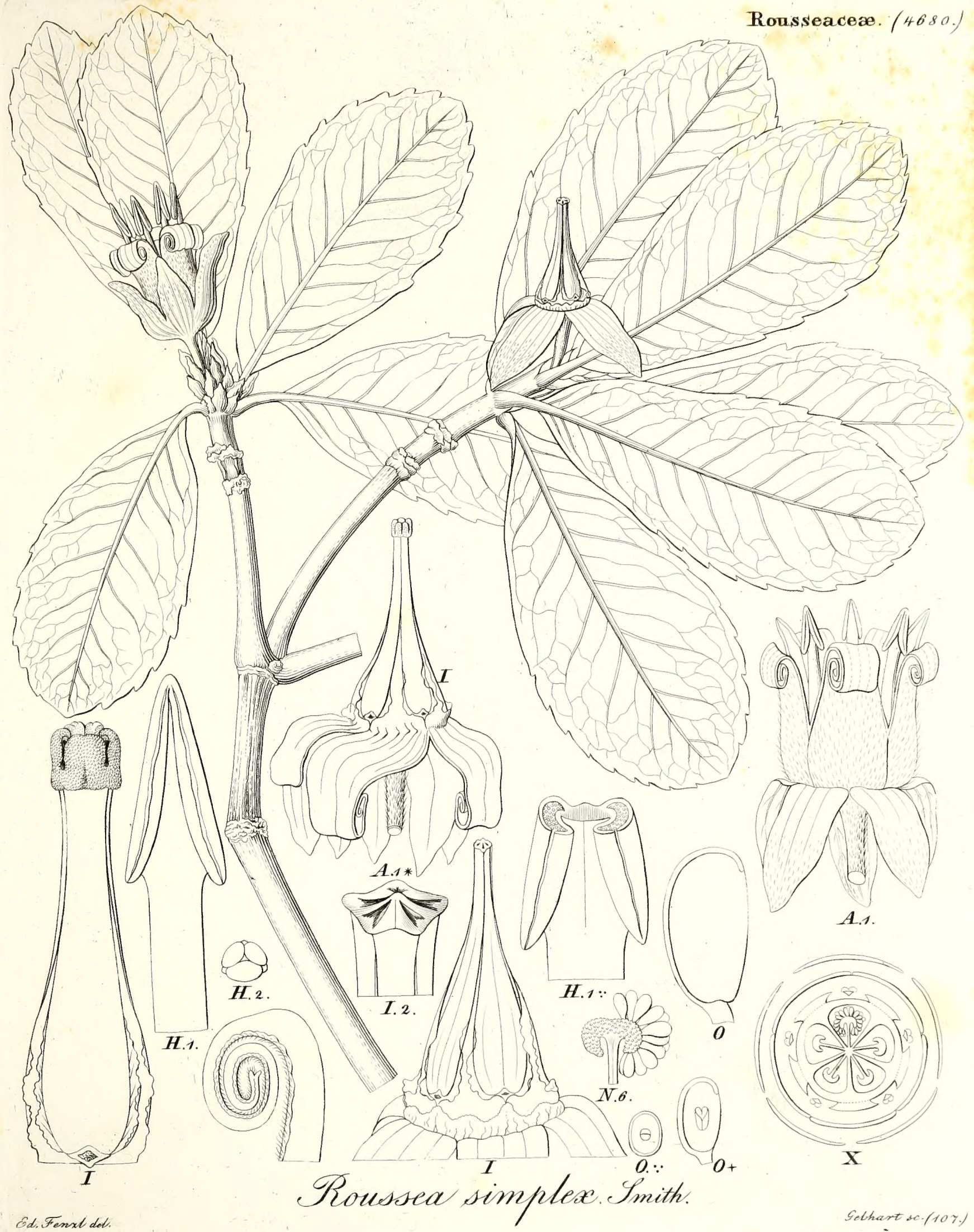


Diotariece.

(5049.)

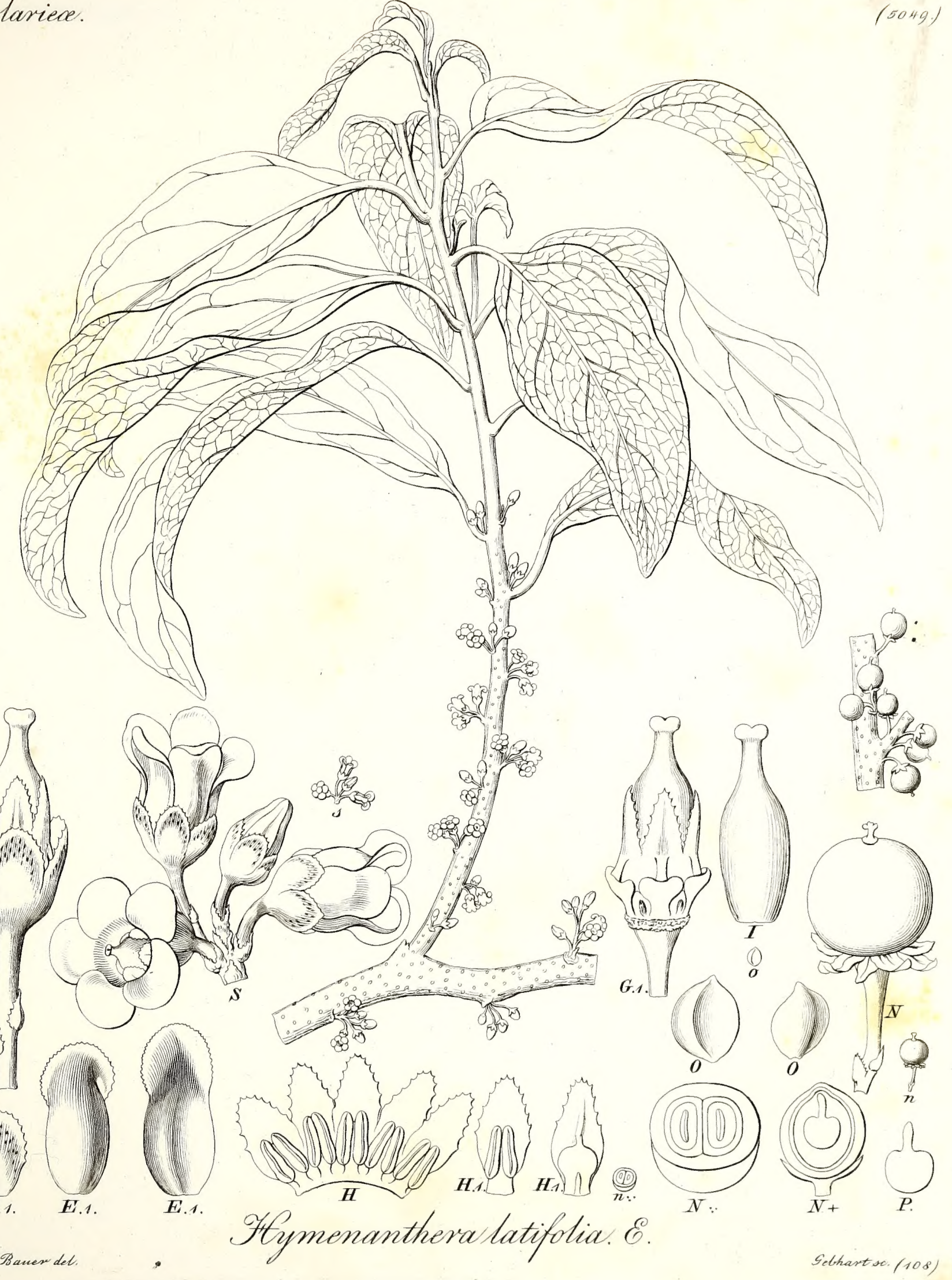


$\begin{gathered}4+4 \\ x+4\end{gathered}=$ 
Haemodoraceade.
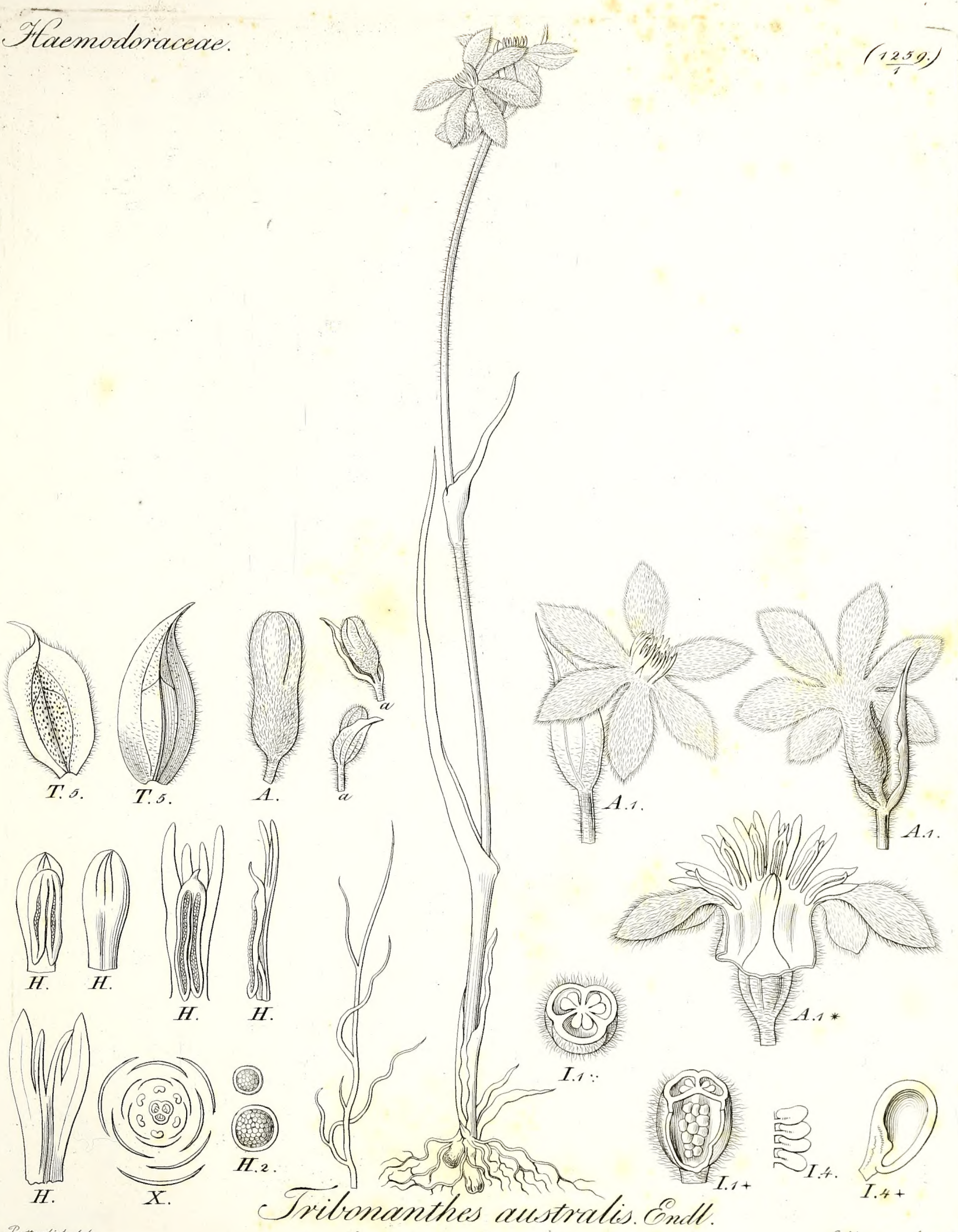


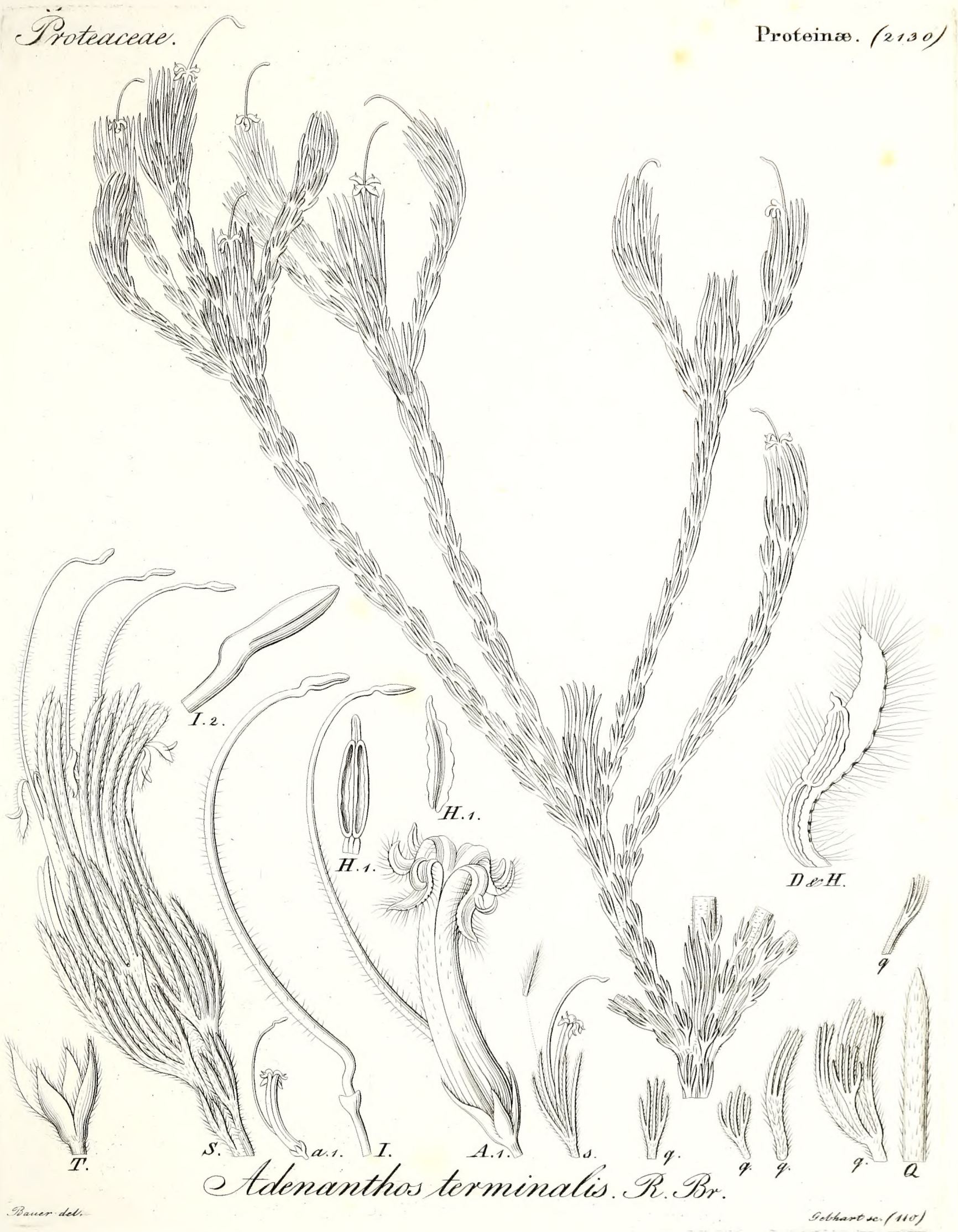




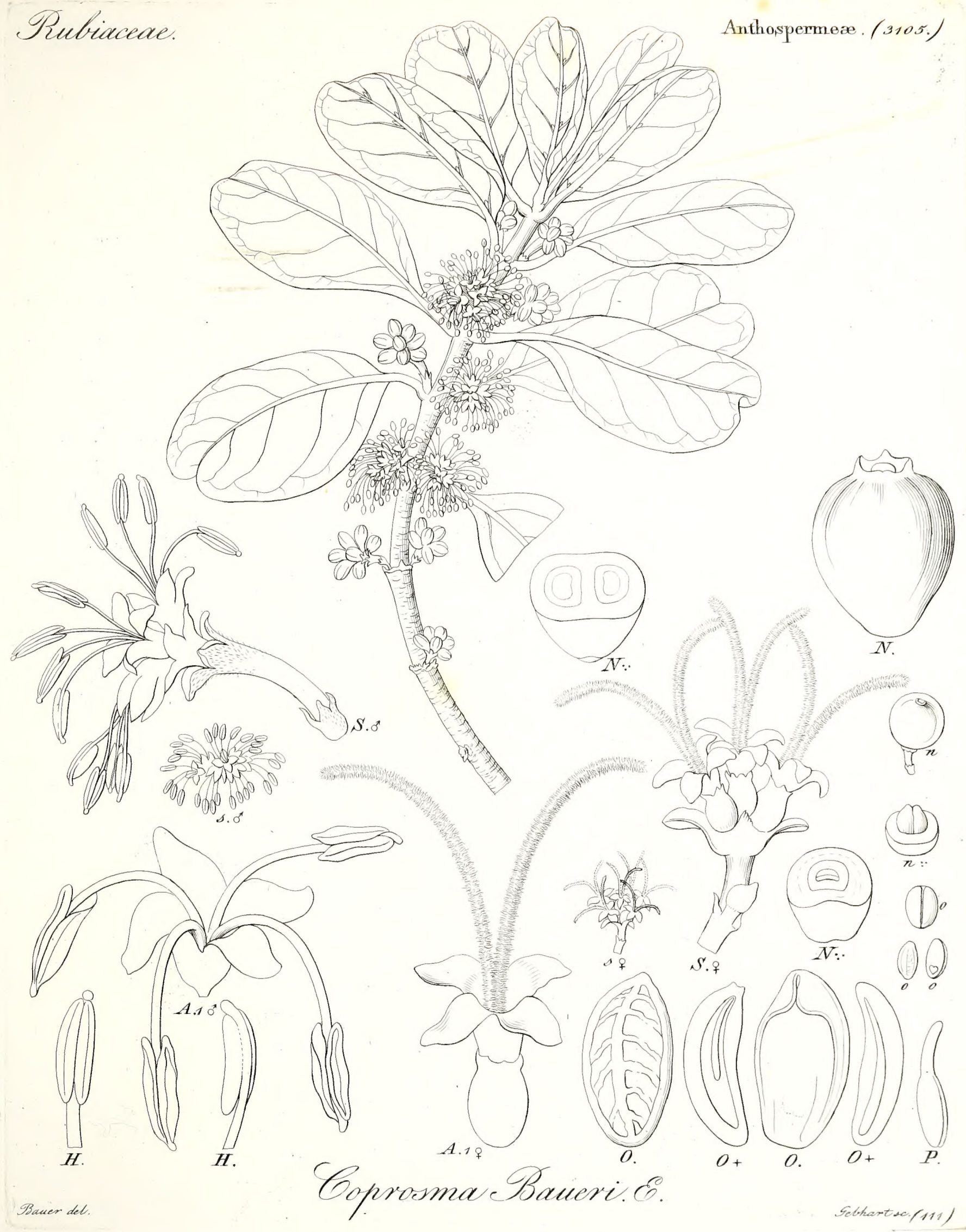


Saxifragaceae.

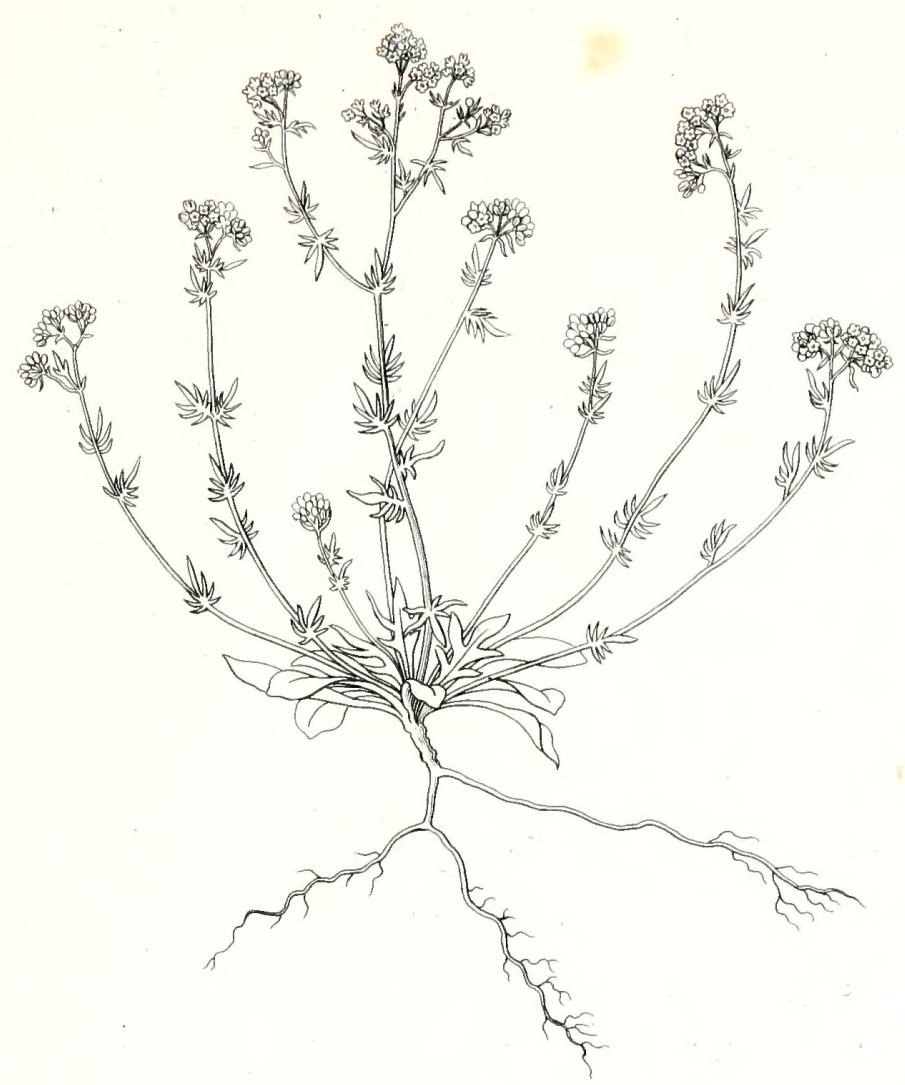

(4629)
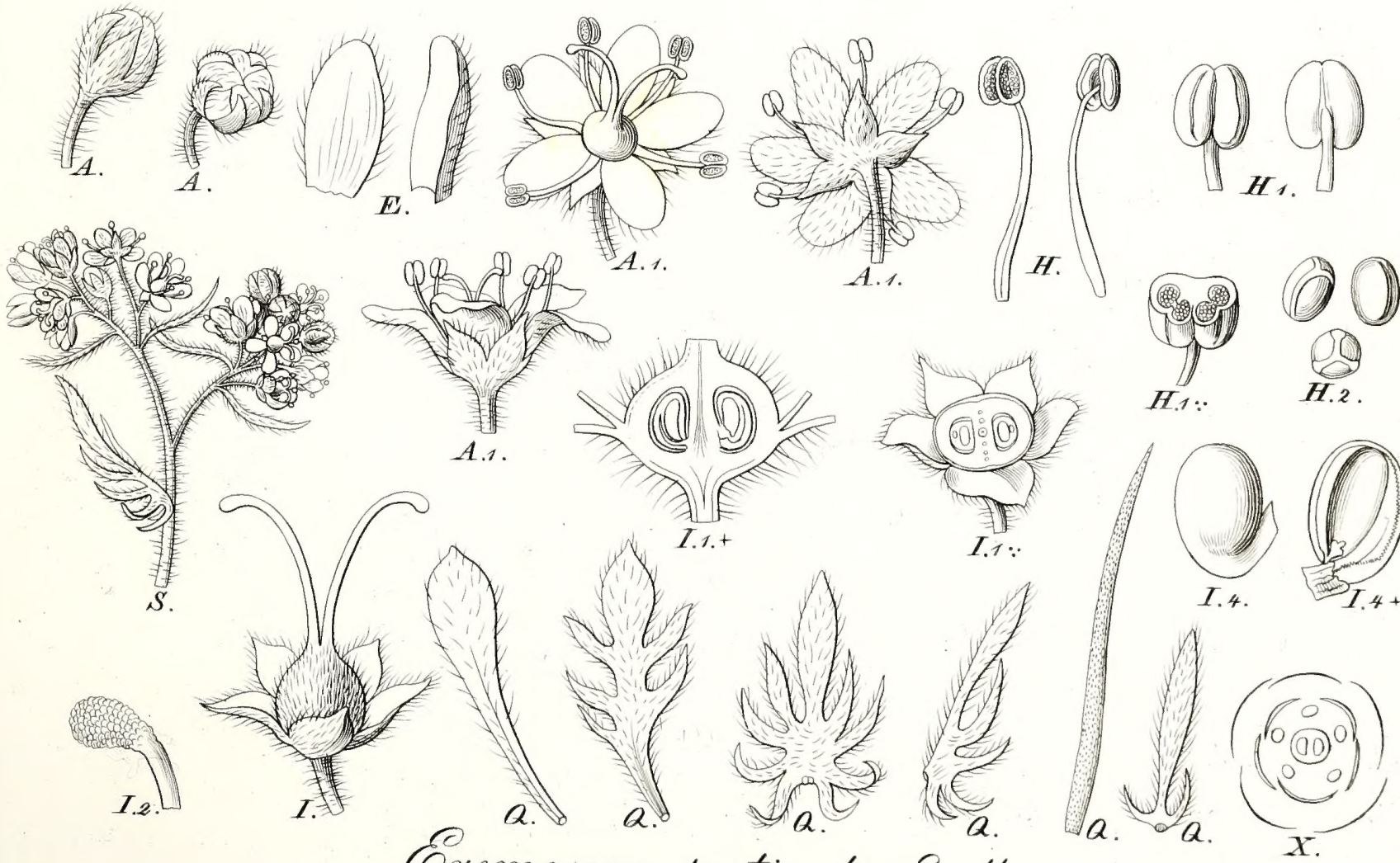
Droseracece.

\section{.}
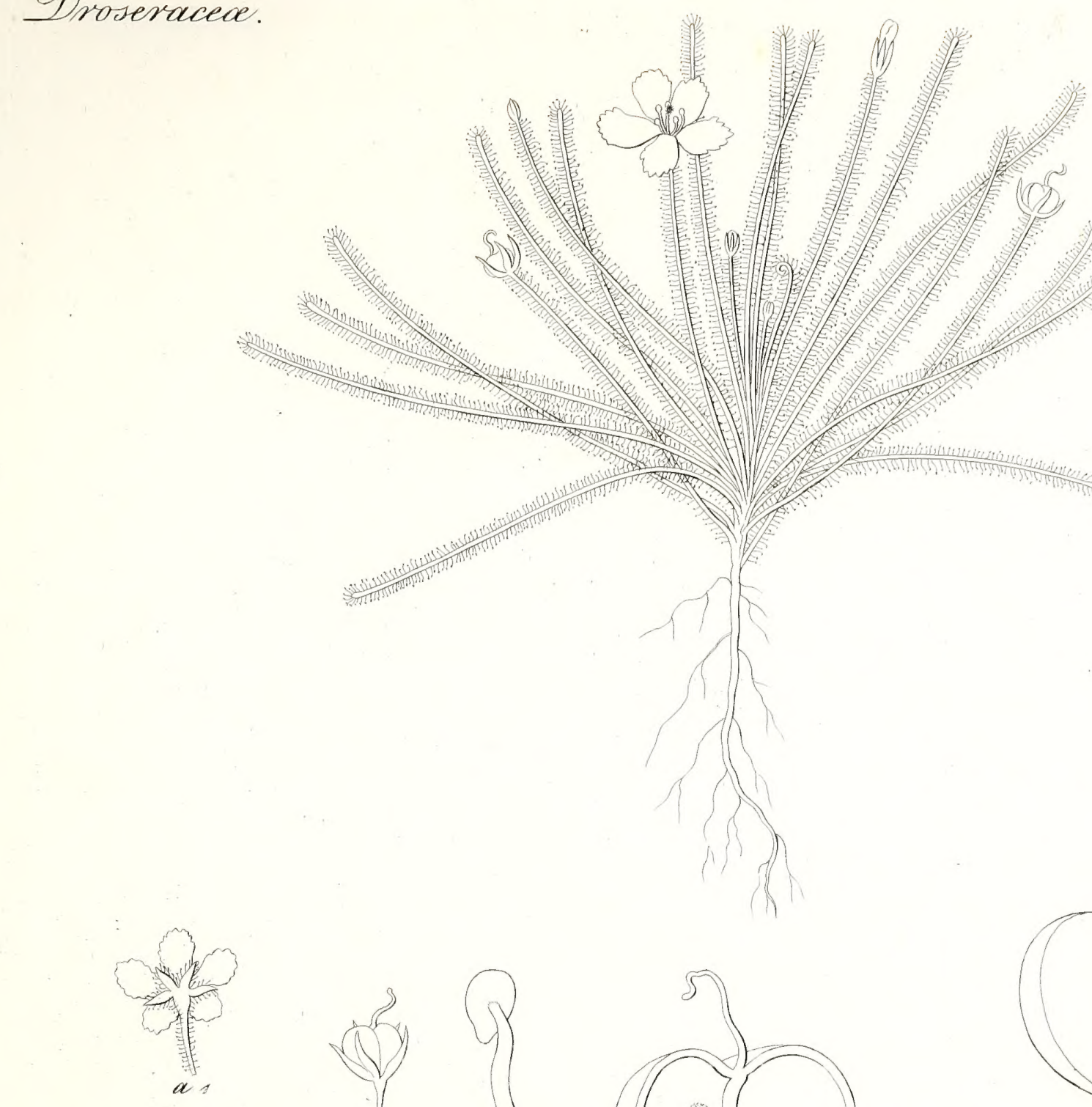
Fissiflorece.

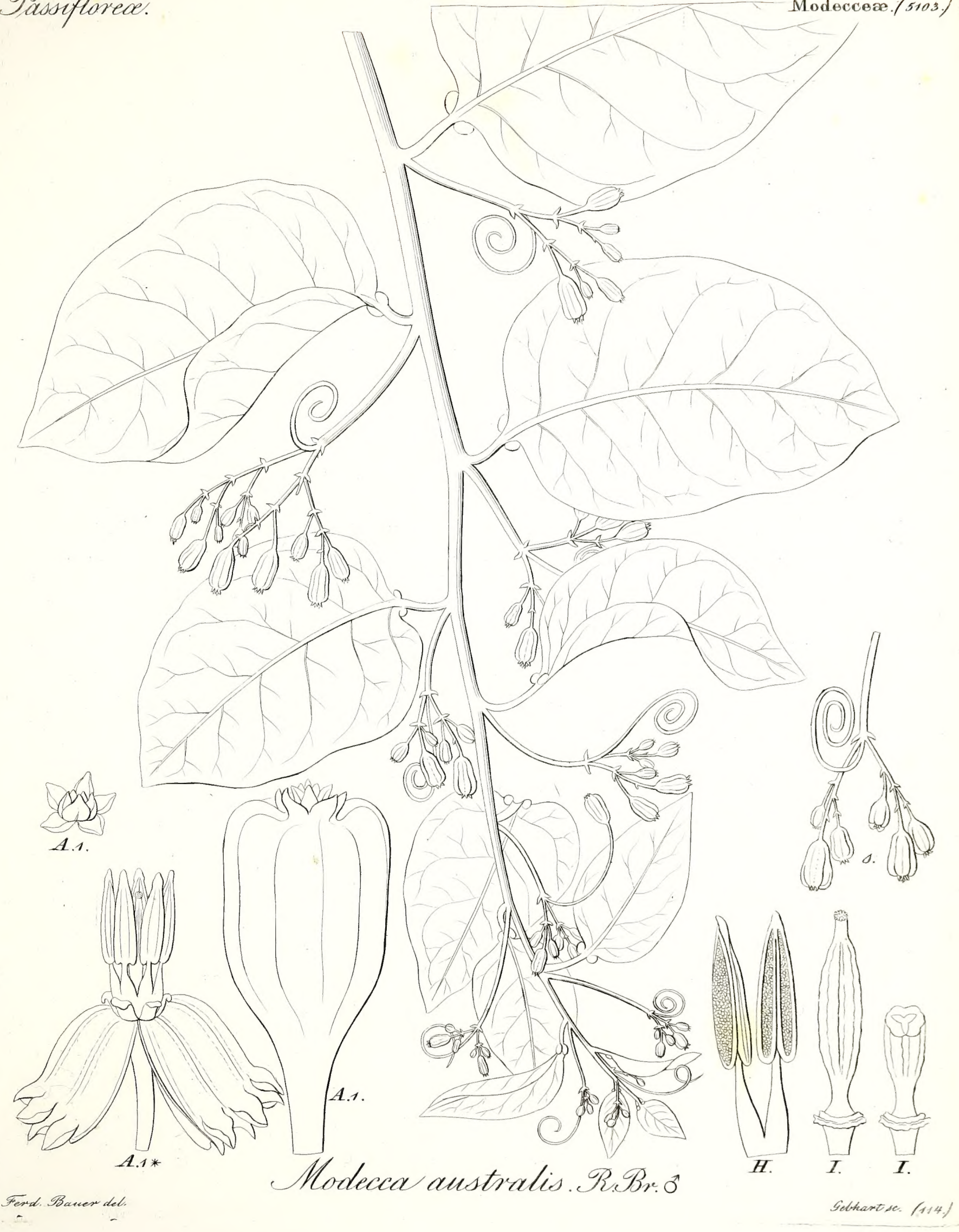


Sassiftorea.

Modecceze.5103.)

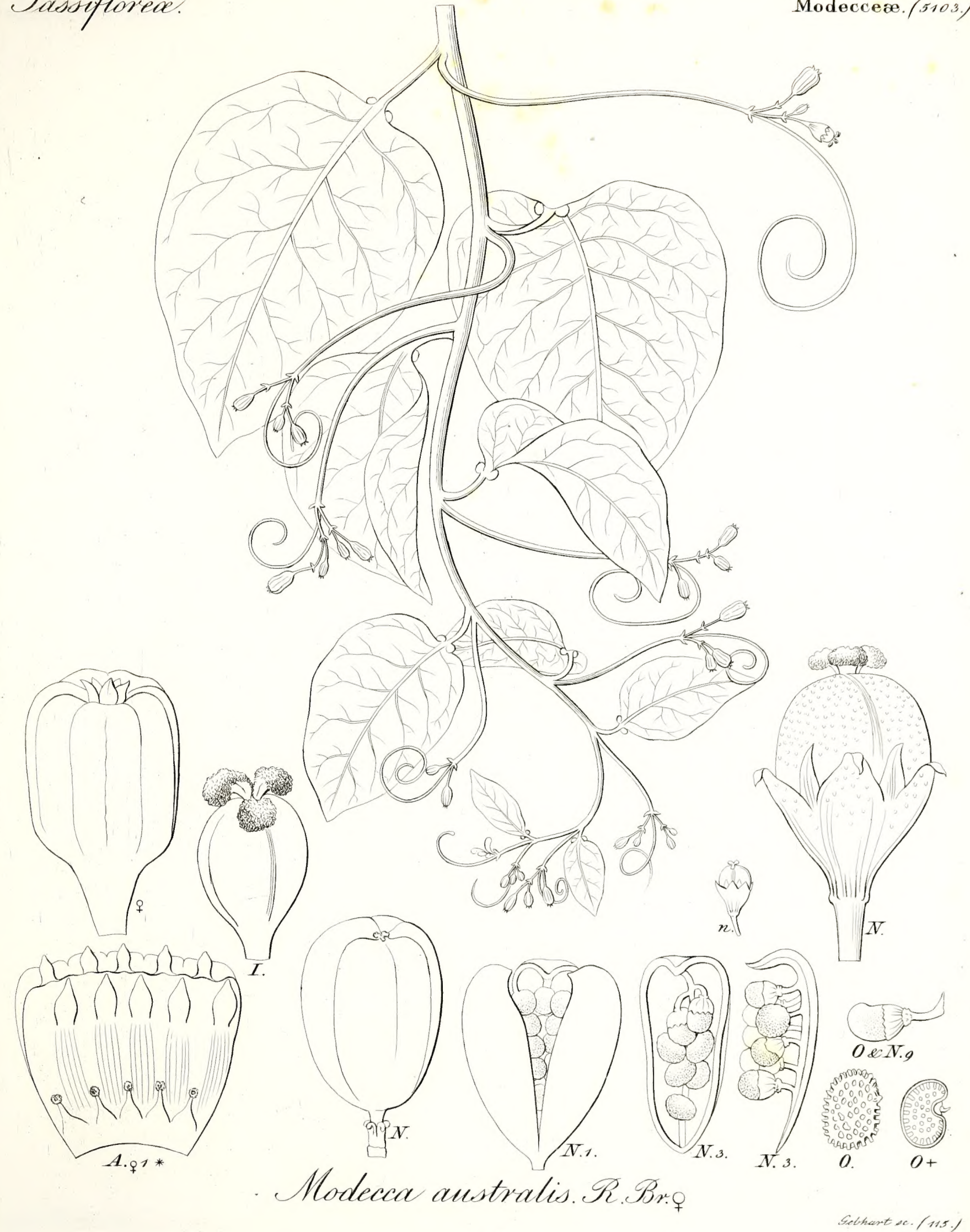



Cucurbitacex.

Melothrieze.(512y.)

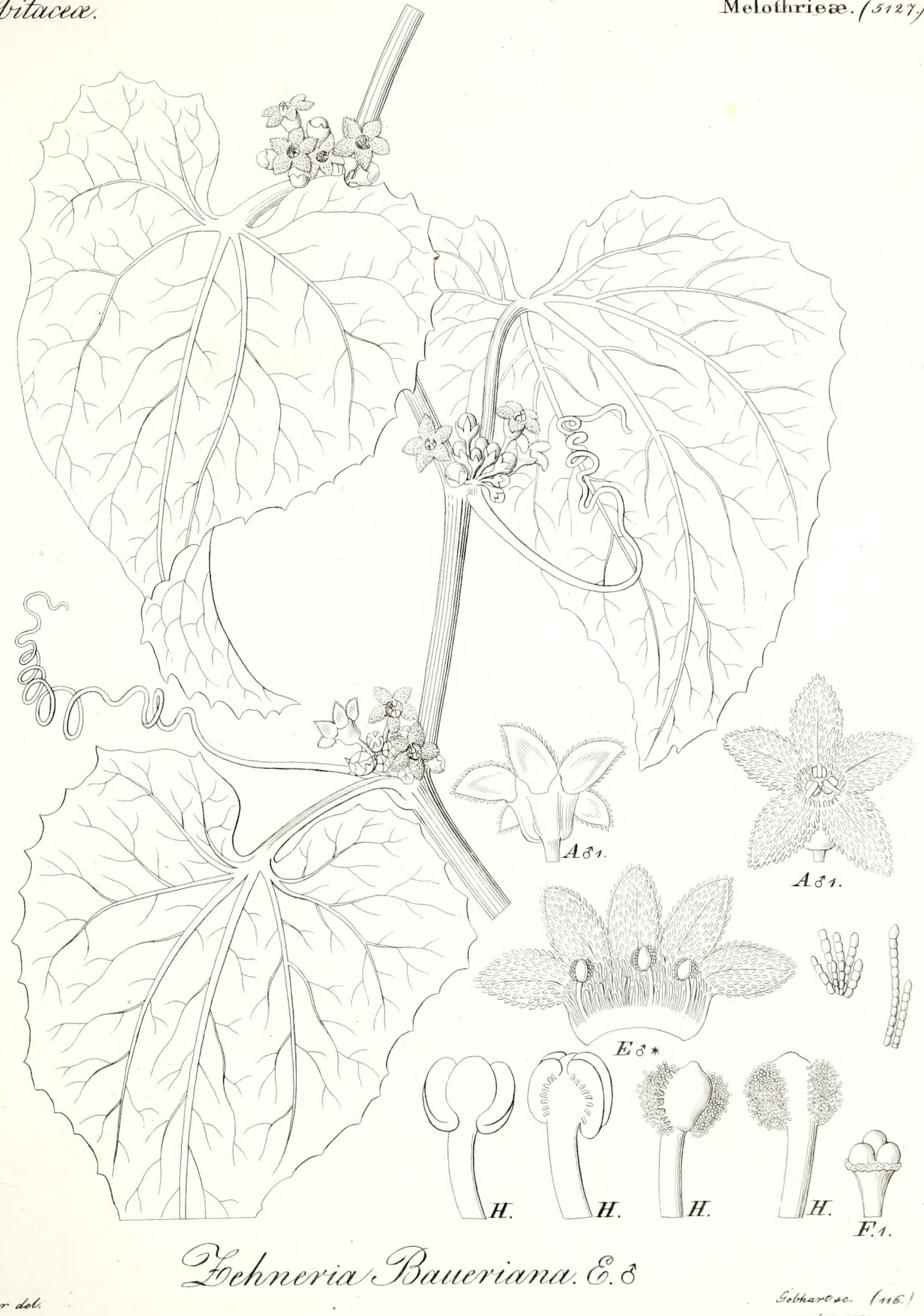





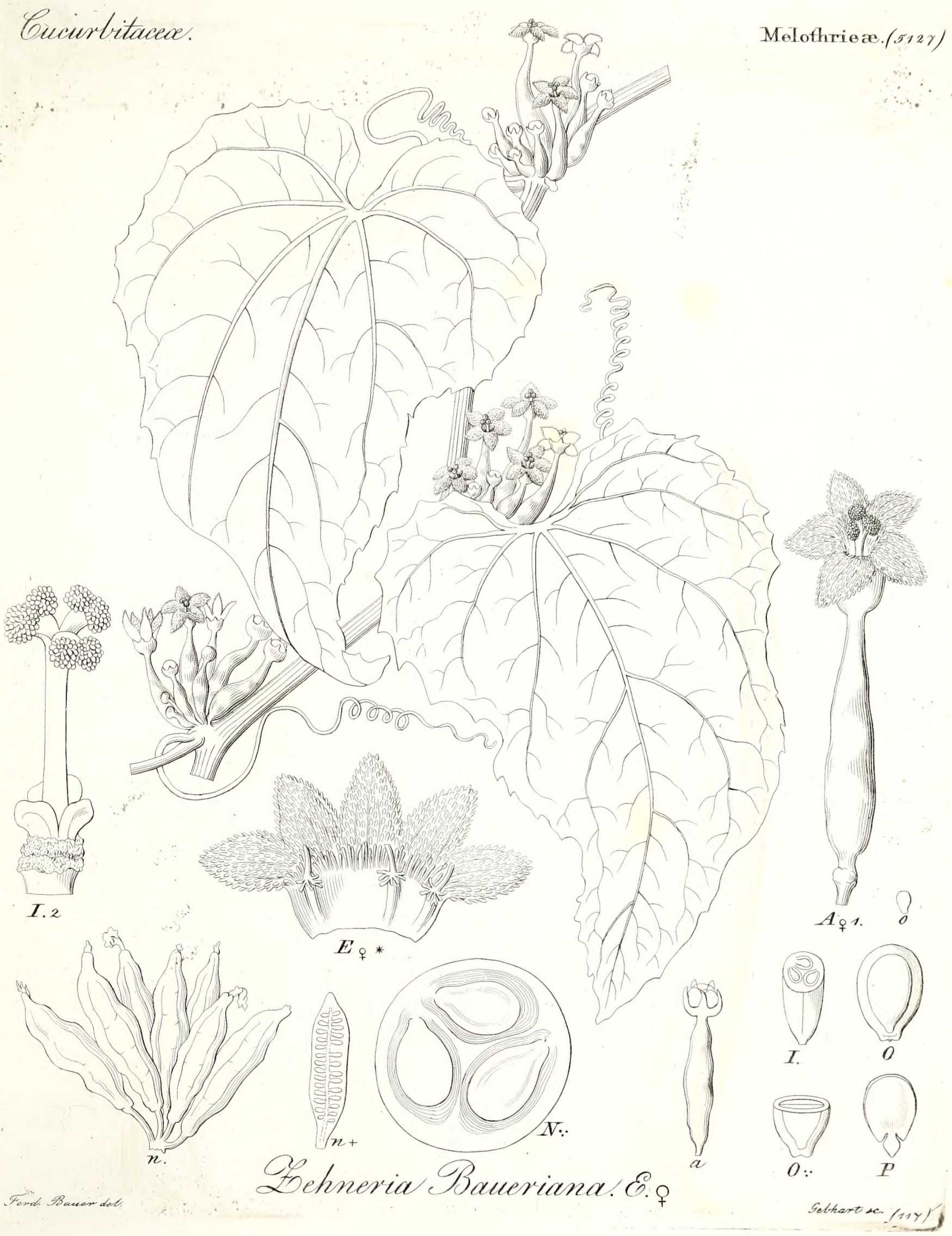


Bütneriacede.

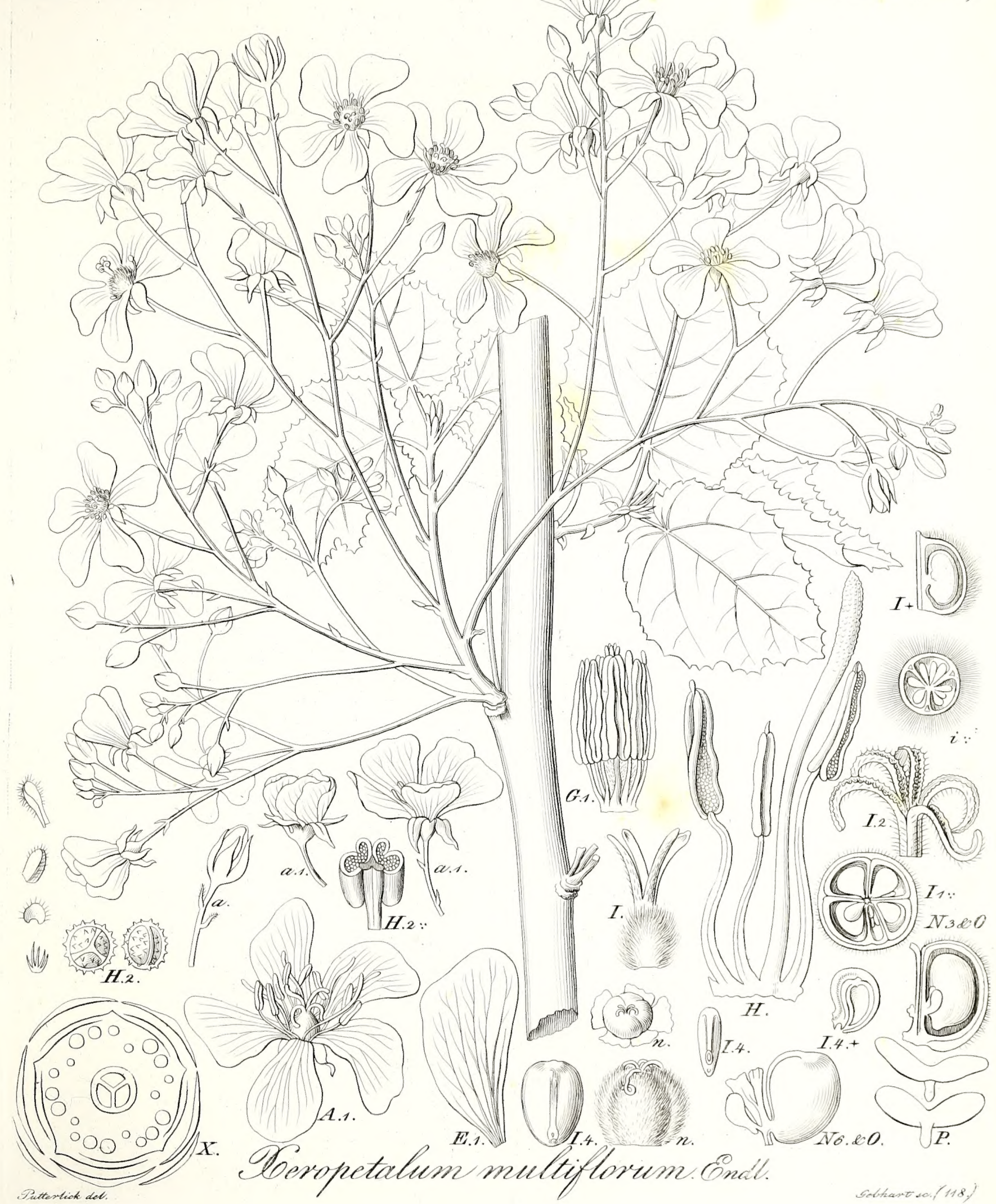


Sapindaceae.

(vin)

$\sin$
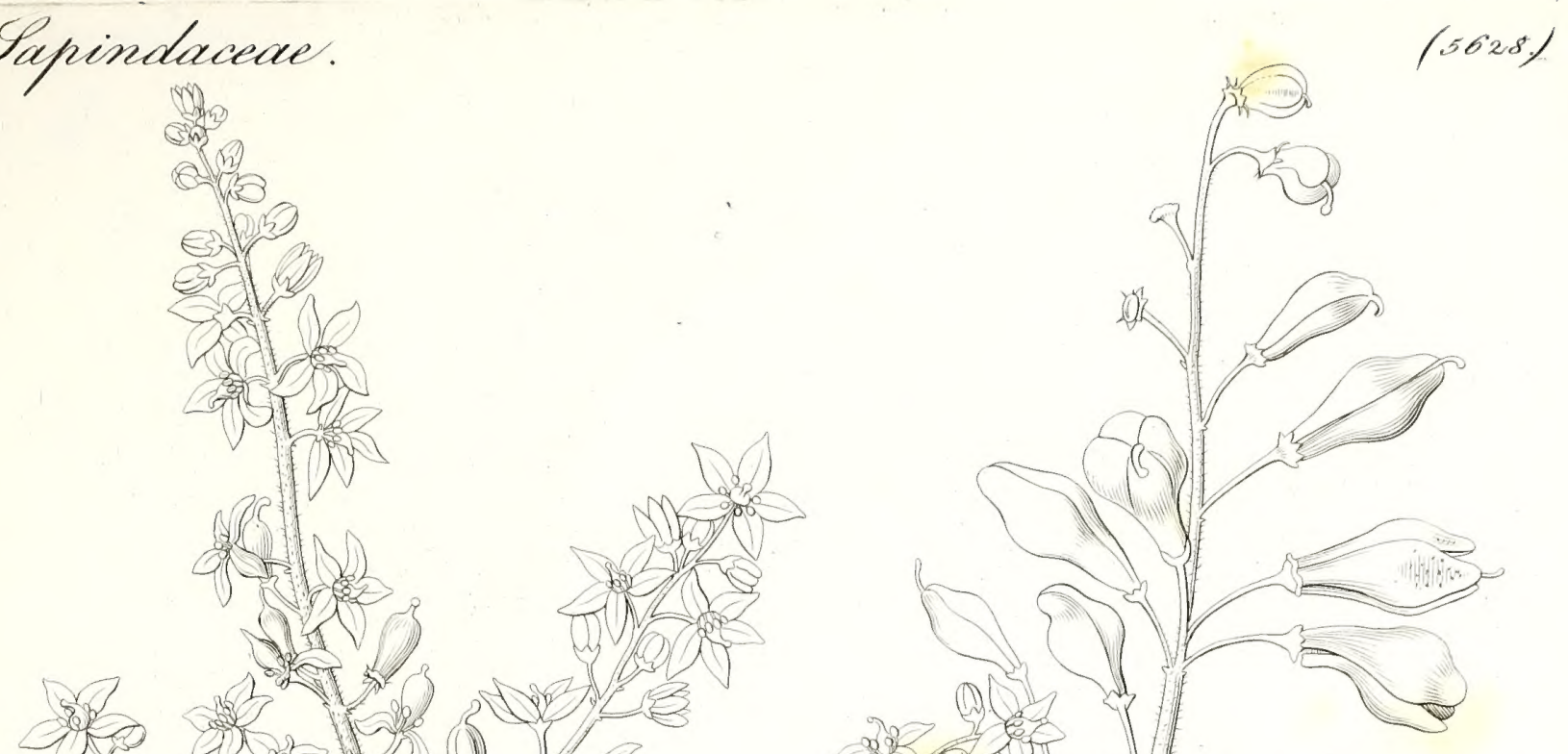

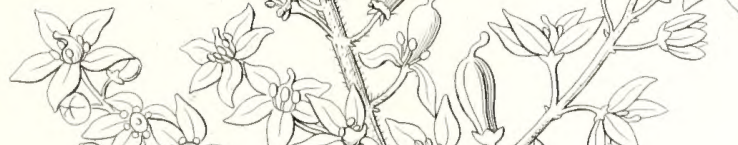
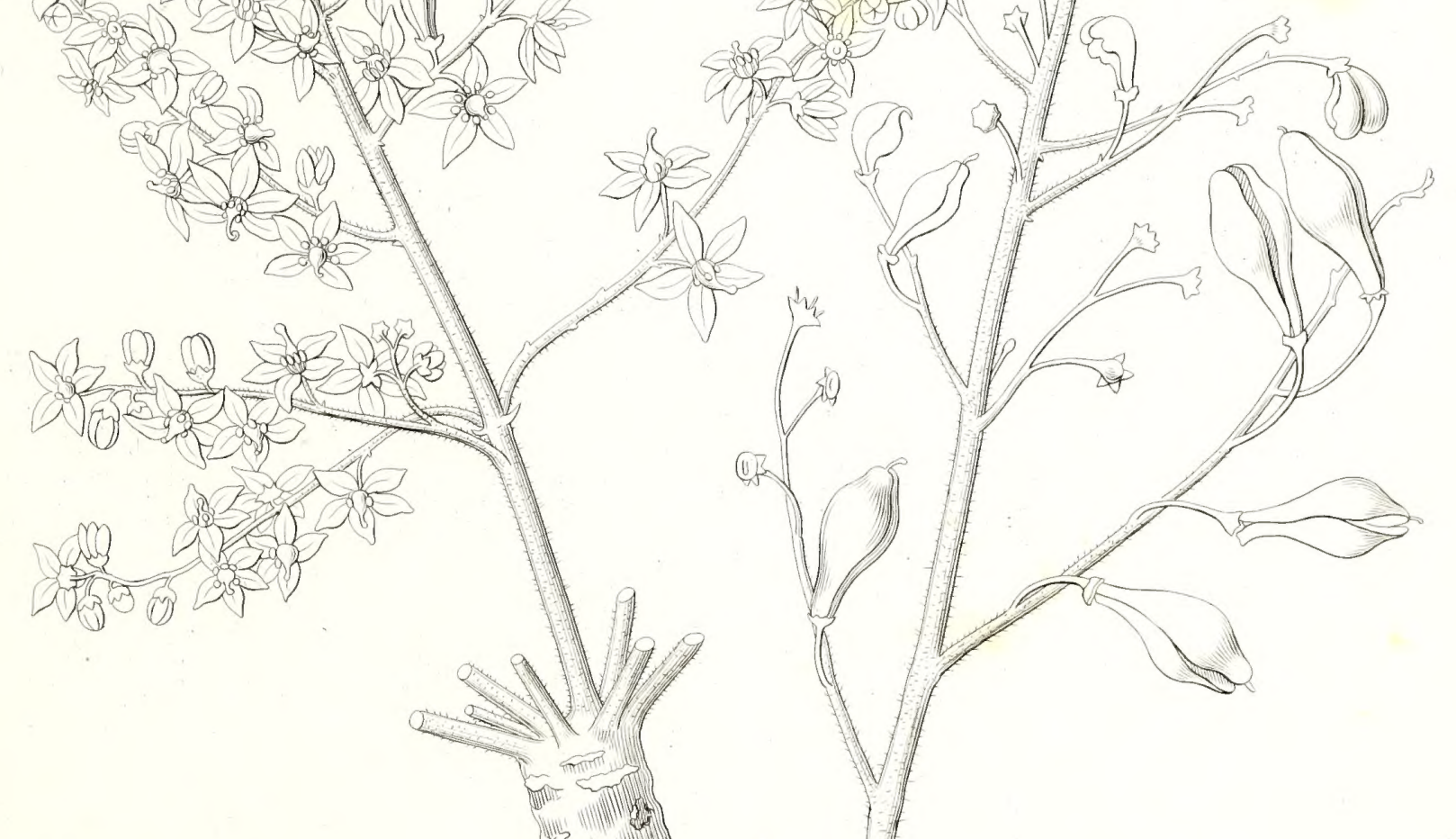

(4)

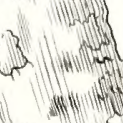

$+\infty$
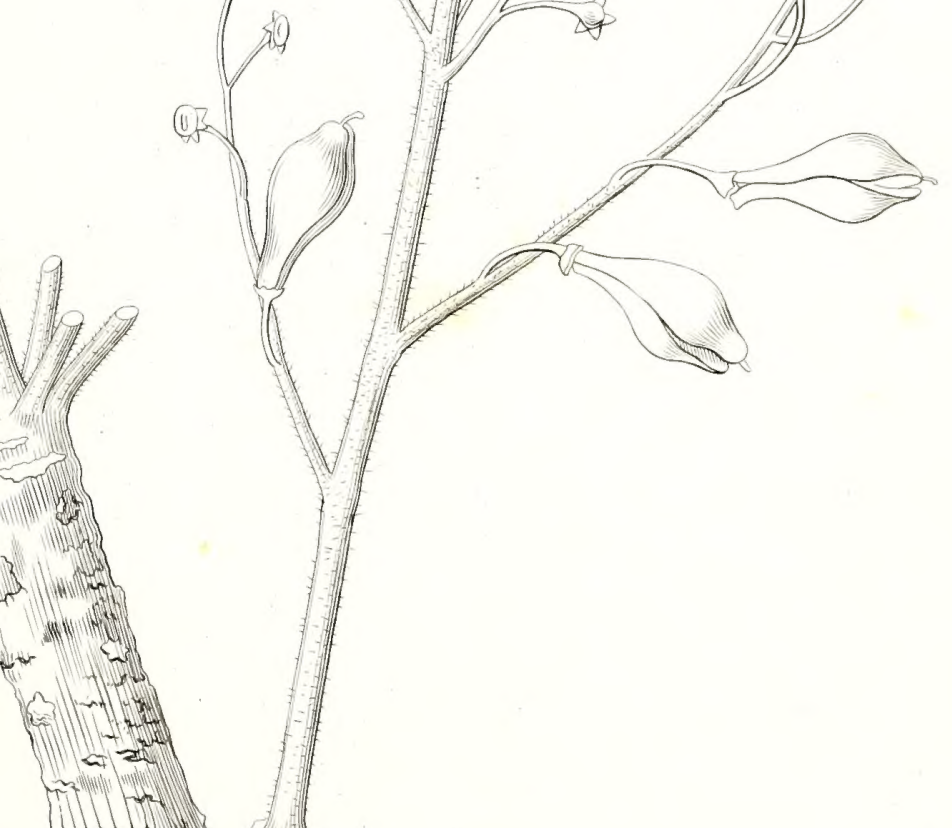


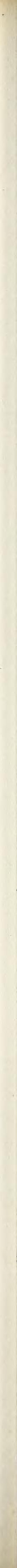


Sapindaceace.

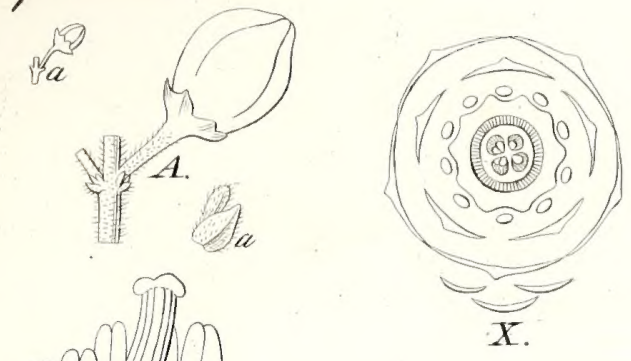

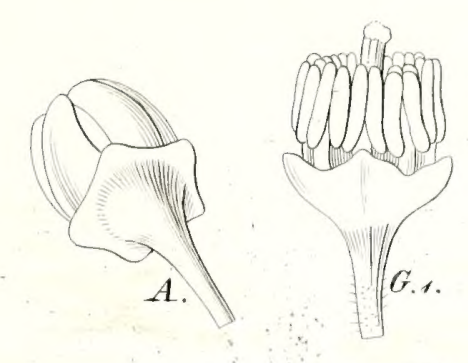

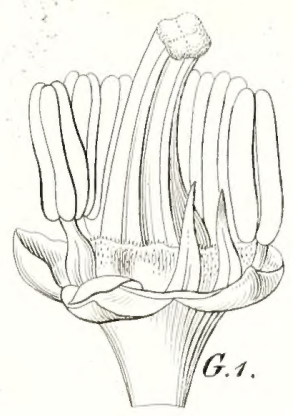

$(5628)$
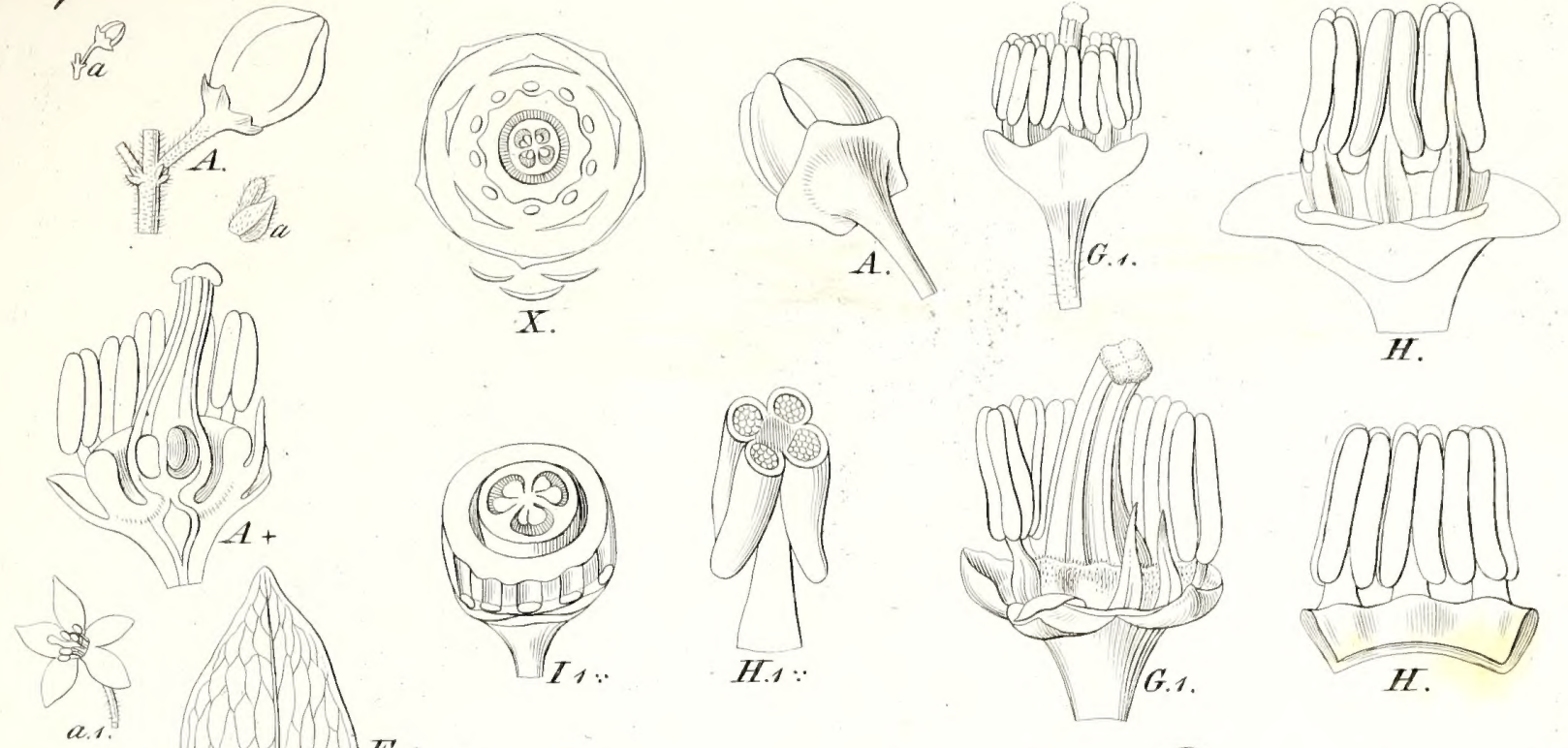

H.
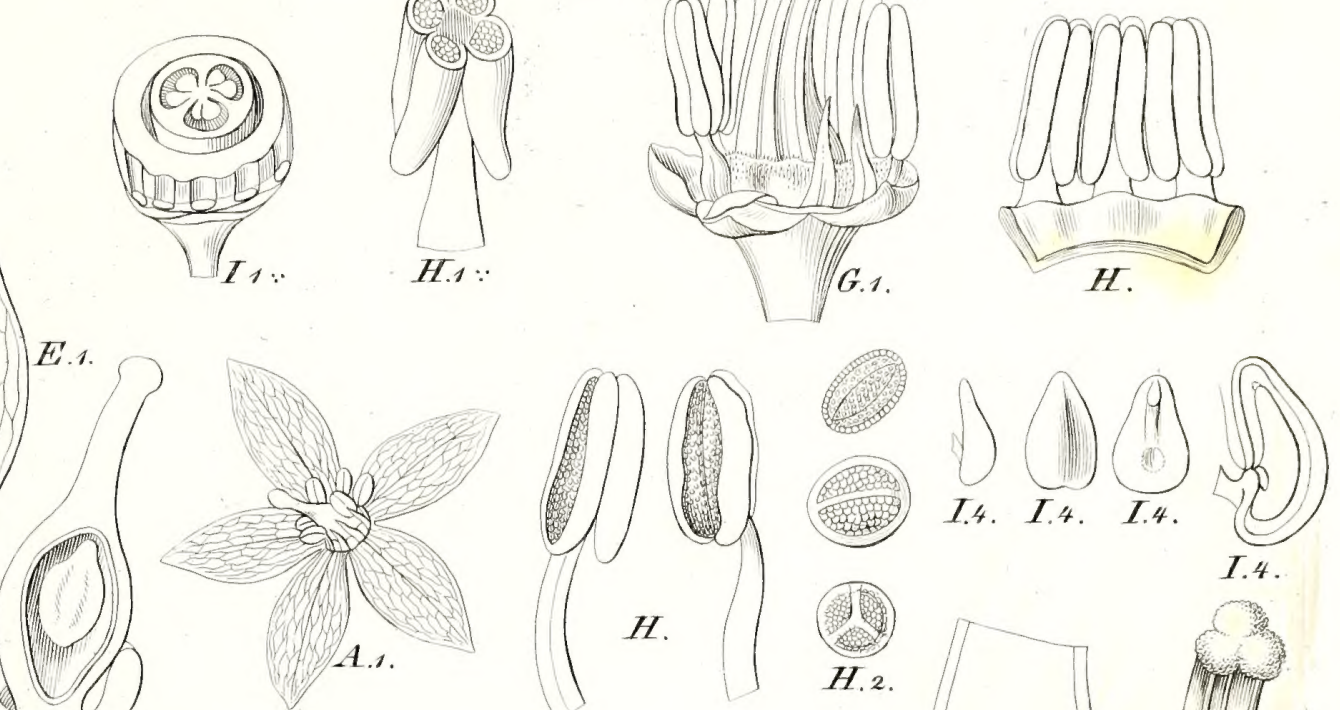

$\underbrace{(10)\}}_{\text {I.1.t }}$
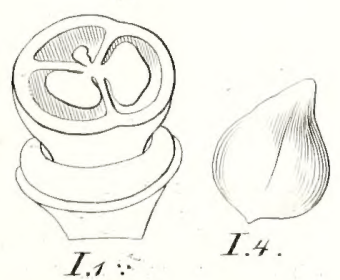

(1.4. I.4. I.t.t
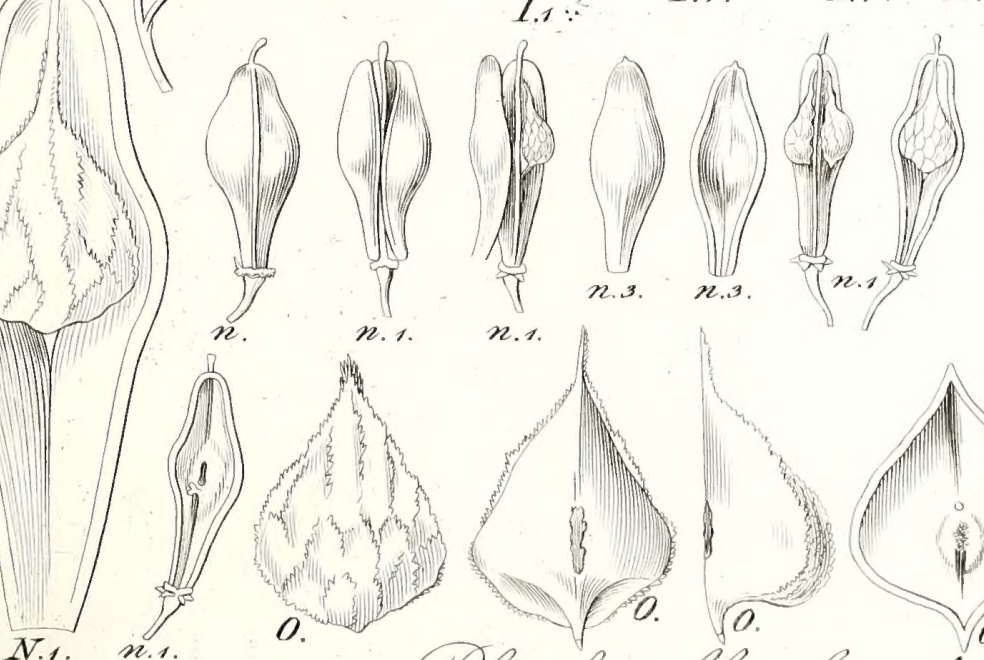

Plosislex floribunda. Endl.
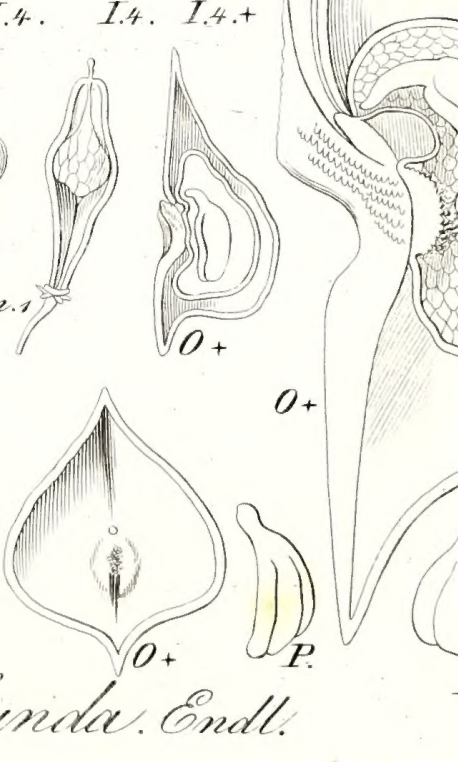
2 
Rhamneis affines.

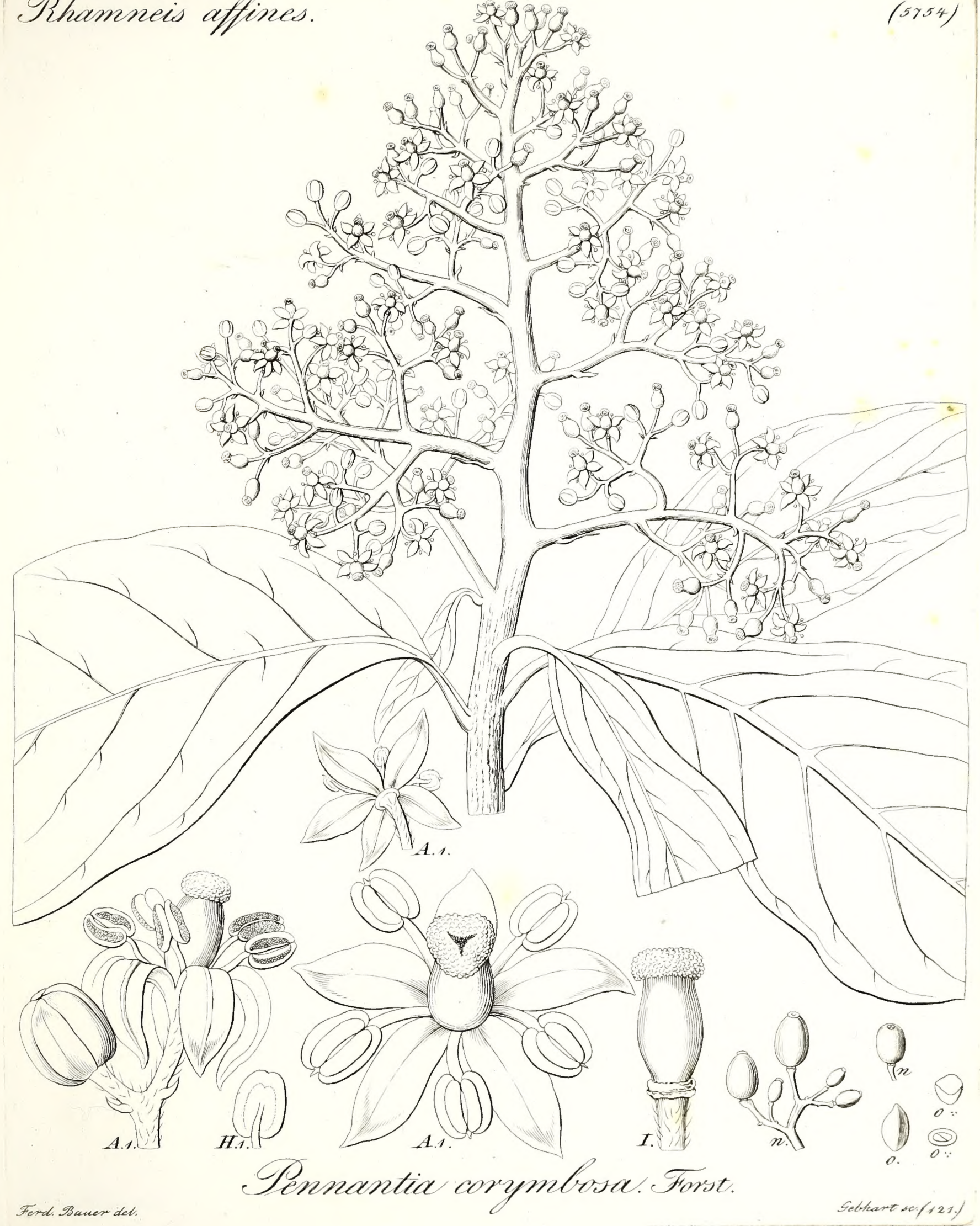





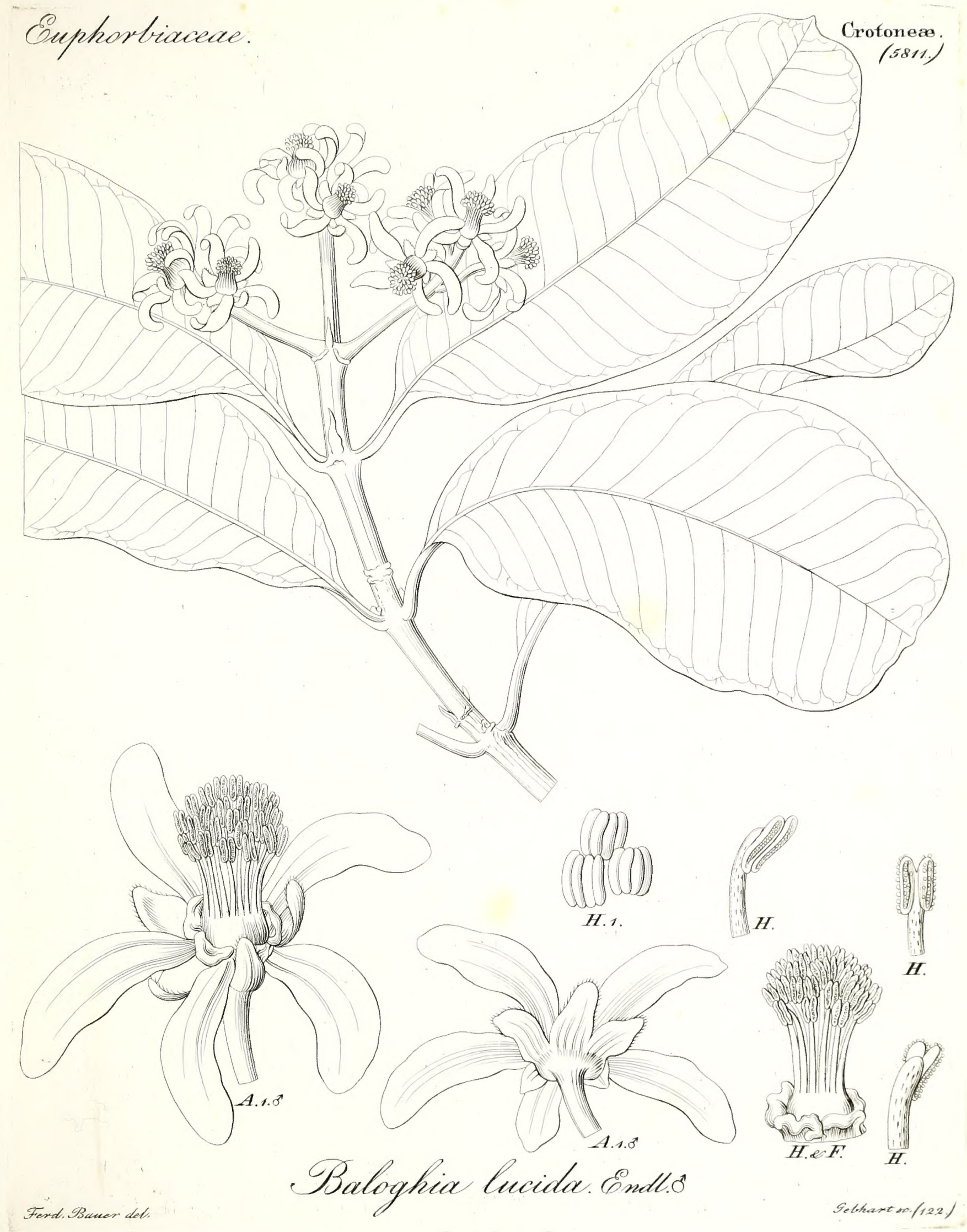




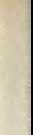

.

(1)

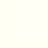

(2) 


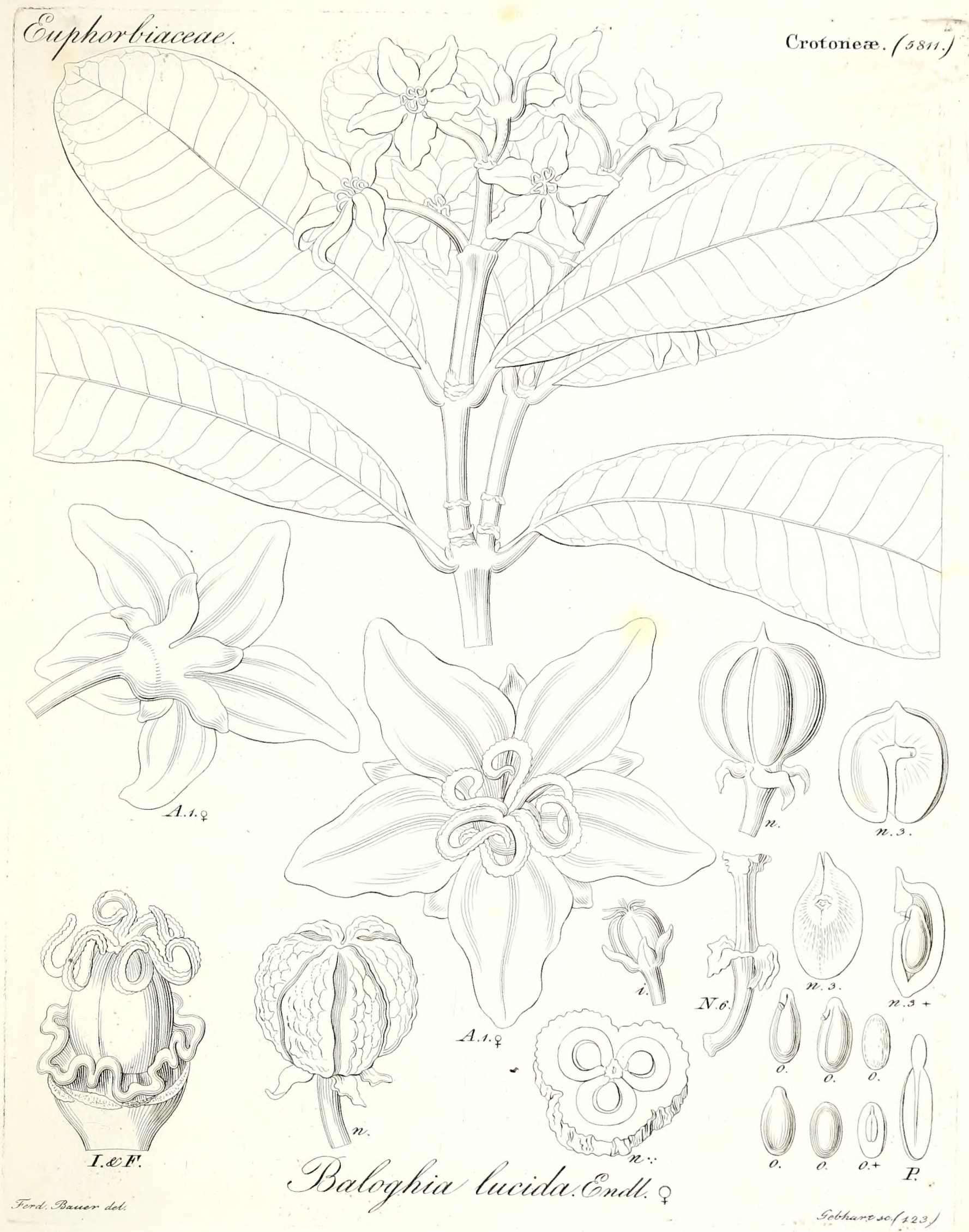



Euphorbiaceae.

Crotonea.(5812.)

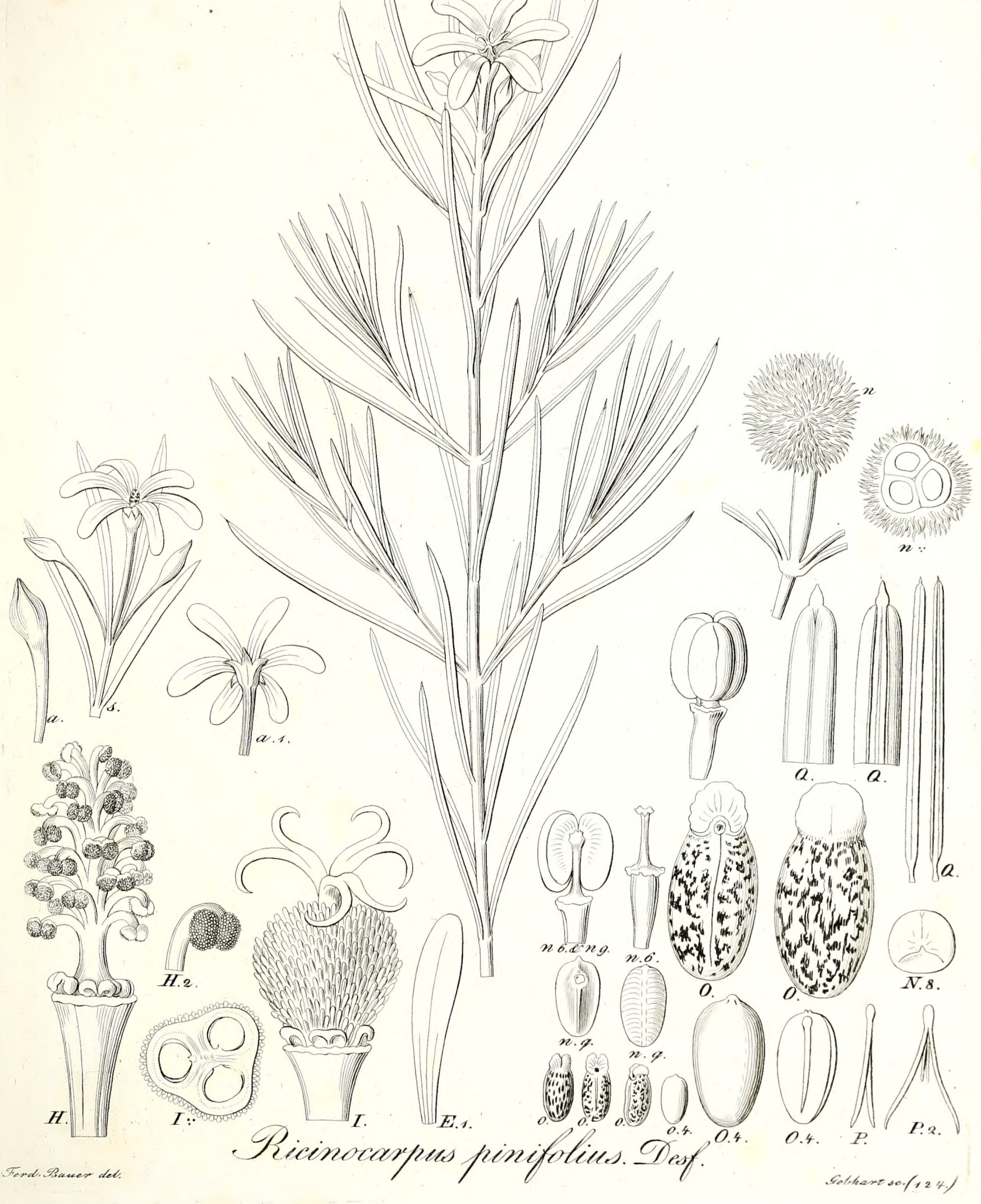




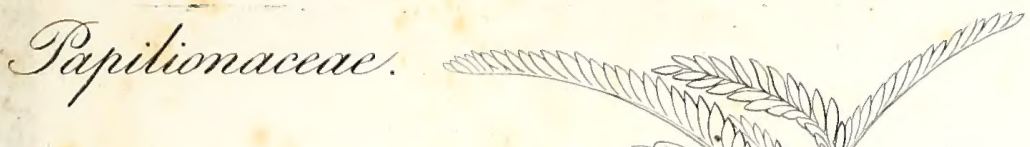

Hedysareas.(060y)

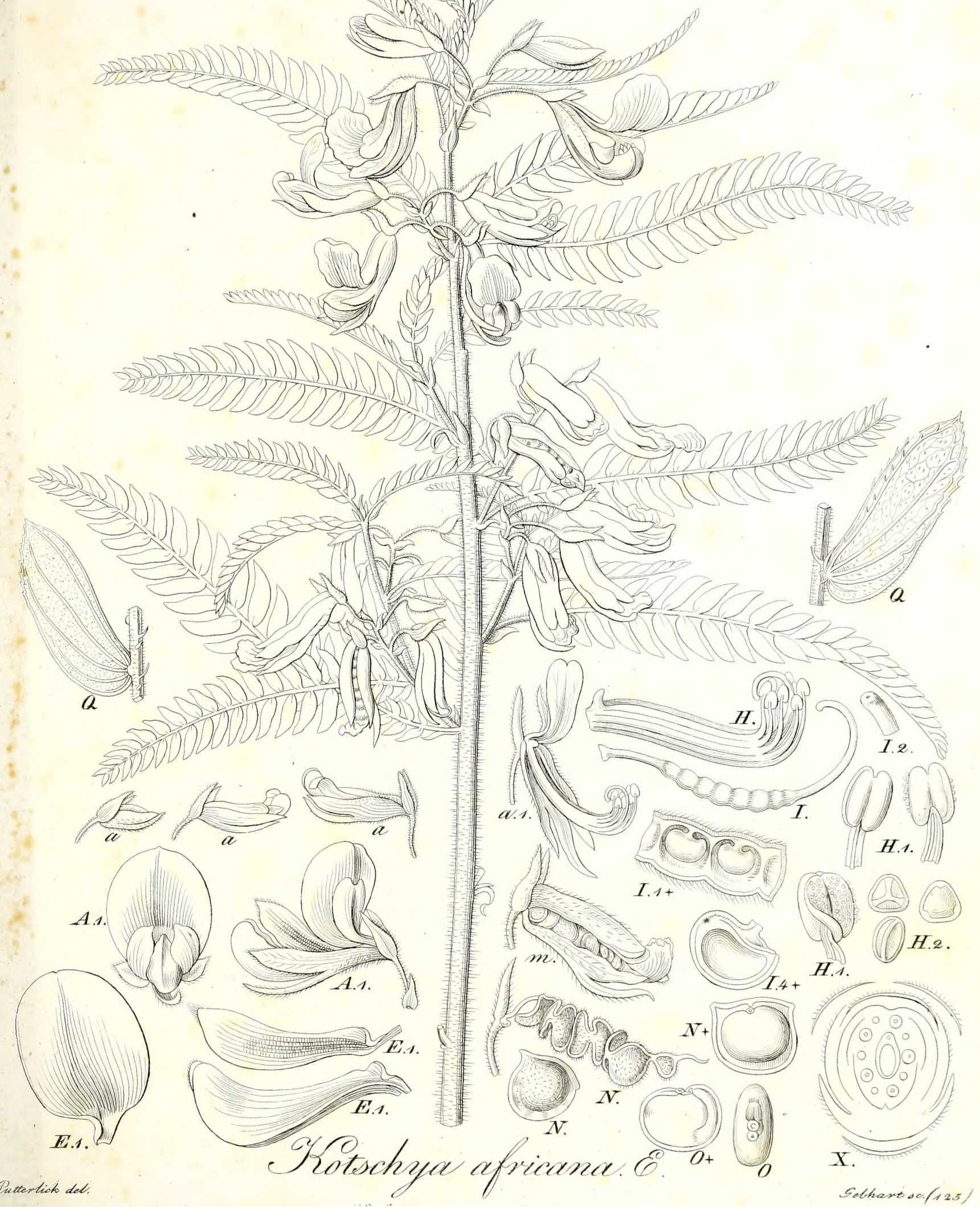




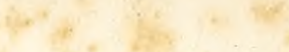

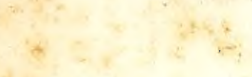

$2+2+2 x^{2}+2+2$

C. 3

$\therefore$ 


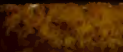





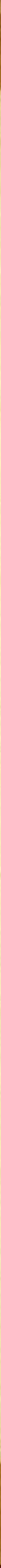


